\title{
Imersões isométricas em 3-variedades Lorentzianas homogêneas
}

\author{
Fernando Manfio
}

\author{
TESE APRESENTADA \\ $\mathrm{AO}$ \\ Instituto DE MATEMÁticA E EstatísticA \\ Universidade DE SÃo PaUlo \\ PARA
OBTENÇÁO DO TÍTULO \\ DOUTOR EM CIÊNCIAS
}

Área de Concentração: Matemática

Orientador: Prof. Dr. Paolo Piccione

Durante o desenvolvimento deste trabalho o autor recebeu auxílio financeiro da CAPES

São Paulo, Maio de 2008 


\title{
Imersões isométricas em 3-variedades Lorentzianas homogêneas
}

\author{
Este exemplar corresponde à redação \\ final da tese devidamente corrigida \\ e defendida por Fernando Manfio \\ e aprovada pela Comissão Julgadora.
}

Banca Examinadora:

- Prof. Dr. Paolo Piccione (orientador) - IME-USP.

- Prof. Dr. Daniel Victor Tausk - IME-USP.

- Prof. Dr. Francesco Mercuri - UNICAMP.

- Prof. Dr. José Miguel Veloso - UFPA.

- Prof. Dr. Levi Lopes de Lima - UFC. 


\section{Agradecimentos}

\section{Sou especialmente grato:}

Aos meus pais, Lino e Cleci, por terem me educado com princípios de ética e por terem me mostrado o caminho do amor e da fraternidade. Agradeço pelo apoio incondicional em todos os momentos.

À minha namorada, Paula, que me acompanhou desde o início nesta trajetória, dividindo comigo todos os momentos. Agradeço pela sua compreensão e por sua paciência durante este tempo, quando, repetidas vezes, deixei sua companhia para passar longas horas estudando.

Aos meus irmãos, Cassio e Caroline, pelo apoio e pela amizade nesta caminhada. Apesar da distância, conseguimos cultivar nossa amizade e compartilhar nossos mundos.

Aos amigos e colegas de pós-graduação que, no decorrer desses últimos anos, compartilharam comigo momentos de alegria, de angústia e de trabalho. Em especial, sou grato ao Sinuê Lodovici, pela amizade e por nossas conversas de Matemática.

Aos professores da pós-graduação do IME que de alguma forma contribuiram na minha formação. Sou profundamente grato ao Daniel Tausk que, por inúmeras vezes, esclareceu minhas dúvidas e me fez indagar sobre outras questões.

Por último quanto à ordem, mas não quanto à importância, expresso a minha mais profunda gratidão por meu orientador Paolo Piccione, pela escolha do tema, pelo incentivo à pesquisa e por tudo o que aprendi nestes últimos anos. Seu profissionalismo e sua impolgação em relação à Matemática são um grande incentivo para continuar nesta caminhada. 


\section{Resumo}

Neste trabalho, provamos um teorema de imersões isométricas em variedades Lorentzianas homogêneas tridimensionais, usando a teoria de $G$ estruturas. Tais variedades são aquelas consideradas na classificação das 3variedades Lorentzianas homogêneas de Dumitrescu e Zeghib ([11]). Provamos também um teorema de rigidez isométrica para hipersuperfícies em variedades semi-Riemannianas com $G$-estrutura infinitesimalmente homogêneas. No caso particular em que o ambiente são variedades semi-Riemannianas produtos do tipo $Q_{c}^{n} \times \mathbb{R}$ ou variedades Riemannianas homogêneas tridimensionais, provamos o mesmo teorema de rigidez isométrica, porém com hipóteses mais fracas.

Palavras-chave: Imersões isométricas, $G$-estruturas, Rigidez isométrica. 


\section{Abstract}

In this work we prove an isometric embedding theorem in homogeneous Lorentzian manifolds of dimension 3, that were recently classified by $\mathrm{Du}$ mitrescu and Zeghib in [11]. We also prove a rigidity result of isometric embeddings of hypersurfaces in semi-Riemannian manifolds endowed with an infinitesimally homogeneous $G$-structure. In the special case that the semi-Riemannian manifolds are produtcs of the type $Q_{c}^{n} \times \mathbb{R}$, or Riemannian homogeneous 3-manifolds, the result is proven under wear assumptions.

Keywords: Isometric embeddings, $G$-structures, isometric rigidity. 


\section{Sumário}

Introdução $\quad$ V

1 Variedades e Grupos de Lie 1

1.1 Variedades diferenciáveis . . . . . . . . . . . . . . . . 1

1.2 Álgebras de Lie . . . . . . . . . . . . . . . . . . 5

1.3 Grupos de Lie . . . . . . . . . . . . . . . . . . 8

1.3.1 Produto semi-direto de grupos de Lie . . . . . . . . . 11

1.3.2 Grupos de Lie nilpotentes . . . . . . . . . . . . . . . . 14

1.3.3 Grupos de Lie solúveis . . . . . . . . . . . . . . . . . 15

1.4 Grupos de Lie clássicos . . . . . . . . . . . . . . . . . . 17

1.5 Variedades homogêneas . . . . . . . . . . . . . . . 21

1.6 Métricas semi-Riemannianas $G$-invariantes . . . . . . . . . 24

2 Espaços Fibrados e Conexões $\quad 30$

2.1 Fibrados principais . . . . . . . . . . . . . . . . 30

2.1.1 Subfibrados principais . . . . . . . . . . . . 33

2.1.2 Morfismos de fibrados principais . . . . . . . . . 35

2.1.3 Pull-back de fibrados principais . . . . . . . . . . 36

2.2 Fibrados vetoriais . . . . . . . . . . . . . . . . . . . . 38

2.2.1 Seções de fibrados vetoriais . . . . . . . . . . . . . . . 42

2.2.2 Morfismos de fibrados vetoriais . . . . . . . . . . . 45

2.2 .3 Pull-back de fibrados vetoriais . . . . . . . . . . . . 46

2.2 .4 Subfibrados vetoriais . . . . . . . . . . . . . 48

2.3 Conexões em fibrados principais . . . . . . . . . . . . 50

2.4 Conexões em fibrados vetoriais . . . . . . . . . . . . 55

2.4.1 Conexão no fibrado vetorial quociente . . . . . . . . 60

2.5 A forma de curvatura . . . . . . . . . . . . . . . 61

2.6 Conexões lineares $G$-invariantes em espaços homogêneos . . . 62

2.6 .1 A conexão de Levi-Civita . . . . . . . . . . . . . 68 
3 G-estruturas e a Inner Torsion $\quad 71$

3.1 Introdução . . . . . . . . . . . . . . . . . . 71

$3.2 G$-estruturas em fibrados vetoriais . . . . . . . . . . . . 72

3.3 A inner torsion de uma $G$-estrutura . . . . . . . . . . . . . 74

3.4 A inner torsion de $G$-estruturas em espaços homogêneos . . . 82

4 Imersões Isométricas $\quad \mathbf{8 4}$

4.1 Introdução . . . . . . . . . . . . . . . . . . . 84

4.2 As componentes de uma conexão . . . . . . . . . . . . 85

4.3 As equações fundamentais . . . . . . . . . . . . . 88

4.4 Variedades Infinitesimalmente homogêneas . . . . . . . . . . 91

4.5 Existência de imersões isométricas que preservam $G$-estrutura 99

5 3-Variedades Lorentzianas $\quad 107$

5.1 Introdução . . . . . . . . . . . . . . . . . . 107

5.1.1 O Teorema de Classificação de Thurston . . . . . . . . 107

5.1.2 O Teorema de Classificação Lorentziana . . . . . . . . 108

5.2 O grupo Nil . . . . . . . . . . . . . . . . . . . 111

5.2 .1 Preliminares . . . . . . . . . . . . . . . . 111

5.2.2 As equações de compatibilidade para superfícies em Nil 114

5.2 .3 Imersões isométricas em Nil . . . . . . . . . . . . . . 117

$5.3 \quad \mathrm{O}$ grupo $\mathrm{SL}_{2}(\mathbb{R}) \ldots \ldots \ldots \ldots$

5.3.1 Preliminares . . . . . . . . . . . . . . . . . . . . . . . 119

5.3.2 As equações de compatibilidade para superfícies em

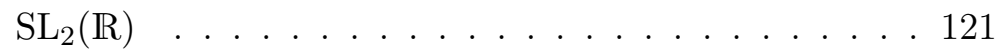

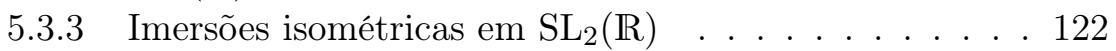

5.4 O grupo Sol . . . . . . . . . . . . . . . . . . . . . . 123

5.4 .1 Preliminares . . . . . . . . . . . . . . . . . . 123

5.4 .2 Imersões isométricas no grupo Sol . . . . . . . . . . 129

6 Rigidez Isométrica 131

6.1 Introdução . . . . . . . . . . . . . . . . . . . . . . 131

$6.2 \quad$ G-rigidez isométrica . . . . . . . . . . . . . . . . 132

$6.3 G$-rigidez isométrica de hipersuperfícies . . . . . . . . . . 133

6.4 G-rigidez isométrica de hipersuperfícies em produtos $Q_{c}^{n} \times \mathbb{R}$ e em 3-variedades . . . . . . . . . . . . . . . . . 137

6.4.1 $G$-rigidez isométrica de hipersuperfícies em $Q_{c}^{n} \times \mathbb{R}$. . 138

6.4.2 G-rigidez isométrica de superfícies em 3-variedades . . 139

$\begin{array}{ll}\text { Referências Bibliográficas } & 140\end{array}$ 


\section{Introdução}

Uma imersão isométrica entre duas variedades Riemannianas $(M, g)$, $(\bar{M}, \bar{g})$ é uma aplicação diferenciável $f: M \rightarrow \bar{M}$, cuja diferencial $\mathrm{d} f_{x}$ : $T_{x} M \rightarrow T_{f(x)} \bar{M}$ é injetora para todo $x \in M$, e tal que

$$
\bar{g}_{f(x)}\left(\mathrm{d} f_{x}(X), \mathrm{d} f_{x}(Y)\right)=g_{x}(X, Y),
$$

para todo $x \in M$ e para quaisquer $X, Y \in T_{x} M$. Dado uma imersão isométrica $f: M \rightarrow \bar{M}$, as relações entre as métricas Riemannianas de $M$ e $\bar{M}$ se exprimem por meio da segunda forma fundamental. Entre estas relações, a mais importante é provavelmente a fórmula de Gauss que generaliza a curvatura Gaussiana de uma superfície regular $S \subset \mathbb{R}^{3}$ e exprime a diferença entre as curvaturas de $M$ e $\bar{M}$ por meio de expressões construídas a partir da segunda forma fundamental. Baseado nas fórmulas de Gauss e Weingarten, são obtidas as equações de Gauss, Codazzi e Ricci da imersão isométrica $f$, que relacionam a curvatura do ambiente com a curvatura da subvariedade, a curvatura do fibrado normal e a segunda forma fundamental (e suas derivadas covariantes). Tais equações são condições necessárias para a existência de imersões isométricas. No caso em que o ambiente $\bar{M}$ é uma variedade Riemanniana de curvatura seccional constante, o clássico Teorema Fundamental para Subvariedades garante que as equações acima são também condições suficientes para determinar unicamente as subvariedades de uma variedade Riemanniana com curvatura seccional constante (ver [7], [33]).

É natural tentar generalizar o Teorema Fundamental para Subvariedades para variedades Riemannianas mais gerais, ou para variedades semi-Riemannianas, ou seja, variedades munidas de um tensor métrico não definido positivo. Dependendo do contexto, condições suficientes para a existência de uma imersão isométrica envolvem hipóteses adicionais. Em [26], os autores desenvolveram uma teoria que unifica vários teoremas de imersões isométricas que aparecem na literatura clássica [7] e também alguns resultados recentes (ver, por exemplo, [9], [10]) sobre existência de imersões isométricas em variedades Riemannianas mais gerais. $\mathrm{O}$ ponto de partida da teoria 
desenvolvida foi a interpretação dessas hipóteses adicionais necessárias em termos de $G$-estruturas e da inner torsion, que é um tipo de derivada covariante da $G$-estrutura. O resultado central de [26] é um teorema de imersões afins em variedades afins infinitesimalmente homogêneas munidas de uma $G$ estrutura. Infinitesimalmente homogênea significa que a curvatura e a torção da conexão, bem como a inner torsion da $G$-estrutura, pode ser escritos unicamente em termos da $G$-estrutura; mais precisamente, são constantes nos referenciais que pertencem a $G$-estrutura.

A fim de entendermos melhor o conceito de homogeneidade infinitesimal, consideremos alguns exemplos. Inicialmente, consideremos o caso em que $\left(M^{n}, g\right)$ é uma variedade Riemanniana, $\nabla$ é a conexão de Levi-Civita de $(M, g), G$ é o grupo ortogonal $\mathrm{O}\left(\mathbb{R}^{n}\right)$ e a $G$-estrutura é dada pelo conjunto dos referenciais ortonormais. Como a conexão $\nabla$ é compatível com $g$, o tensor de torção de $\nabla$ e a inner torsion dessa $G$-estrutura são nulos. A condição para que o tensor de curvatura seja constante nos referenciais ortonormais é equivalente à condição de que $M$ tenha curvatura seccional constante. Neste caso, portanto, o teorema de [26] reproduz o clássico Teorema Fundamental para Subvariedades em variedades Riemannianas de curvatura seccional constante.

Outro exemplo interessante de $G$-estrutura considerado em [26] é o caso de variedades Riemannianas munidas de um campo de Killing $\xi$. Aqui $G$ é o grupo ortogonal $\mathrm{O}\left(\mathbb{R}^{n-1}\right)$, e a $G$-estrutura consiste de todos os referenciais ortonormais cujo último vetor é $\xi$. Neste caso, os autores obtêm um teorema de imersão isométrica em variedades Riemannianas com a propriedade de que o tensor de curvatura e a derivada covariante do campo vetorial em um ponto arbitrário $x$ podem ser escritos somente em termos da métrica Riemanniana em $x$ e do vetor $\xi(x)$. Este é o caso de exemplos importantes como, por exemplo, todas as variedades que são produtos Riemannianos de uma forma espacial com uma cópia de $\mathbb{R}$, bem como todas as variedades Riemannianas homogêneas, simplesmente conexas, tridimensionais, cujo grupo de isometrias tem dimensão 4. Estes exemplos estão considerados em [9] e [10].

Outros dois exemplos são considerados. Primeiro, os autores consideram imersões isométricas em grupos de Lie munidos de uma métrica semiRiemanniana invariante à esquerda. Tais variedades têm uma 1-estrutura natural, dada pela escolha de um referencial ortonormal invariante à esquerda. Claramente o tensor de curvatura é constante neste referencial e a inner torsion da 1-estrutura é simplesmente o tensor de Christoffel associado a este referencial, que também é constante. Outro exemplo considerado é o caso de imersões isométricas em produtos de variedades com curvatura 
seccional constante. Neste caso, a $G$-estrutura considerada consiste dos referenciais ortonormais adaptados a tal produto. Mais geralmente, produtos de variedaes afins infinitesimalmente homogêneas com $G$-estruturas são infinitesimalmente homogêneas.

Um dos objetivos deste trabalho é provar um teorema de imersão isométrica em 3-variedades Lorentzianas homogêneas. Mais precisamente, usamos a teoria desenvolvida em [26], interpretando as condições suficientes para existência de imersões isométricas, para provar um teorema de imersão isométrica em variedades Lorentzianas homogêneas tridimensionais (cf. Teoremas 5.2.7, 5.3.7 e 5.4.5).

Antes de explicarmos o enunciado dos Teoremas, faremos uma pequena discussão a título de motivação. A teoria de classificação das variedades Riemannianas tridimensionais foi estabelecida por Thruston (ver [34]), onde o autor prova que existem apenas oito geometrias Riemannianas maximais. Em [30], Scott descreve cada uma dessas oito geometrias. Mais precisamente, o teorema de classificação de Thruston diz que qualquer geometria tridimensional maximal, simplesmente conexa e que admite um quociente compacto é equivalente a uma das geometrias $(\operatorname{Iso}(X), X)$, onde $X$ é uma das seguintes variedades: $\mathbb{R}^{3}, \mathbb{H}^{3}, \mathbb{S}^{3}, \mathbb{S}^{2} \times \mathbb{R}, \mathbb{H}^{2} \times \mathbb{R}, \widetilde{\mathrm{SL}_{2}(\mathbb{R})}$, Nil ou Sol (ver [30, Teorema 5.1]). Tais variedades são caracterizadas por seu grupo de isometrias ter dimensão 3, 4 ou 6 (ver maiores detalhes no capítulo 5).

O caso Lorentziano, em analogia ao caso Riemanniano e no qual estamos interessados, tem sido estudado recentemente. Em [11], os autores provam que existem somente quatro geometrias Lorentzianas maximais. Mais precisamente, toda geometria Lorentziana maximal, compacta, conexa, tridimensional, é equivalente a uma das seguintes geometrias: Minkowski, antide Sitter, Heisenberg-Lorentz ou Sol-Lorentz. Os modelos de tais geometrias são $\mathbb{R}^{3}, \widetilde{\mathrm{SL}_{2}(\mathbb{R})}$, Nil ou Sol, respectivamente. Nosso interesse reside nos grupos de Lie Nil e Sol, por não terem curvatura seccional constante.

O grupo de Heisenberg Nil (cf. Exemplo 1.4.8) é considerado com a métrica Lorentziana invariante à esquerda

$$
g=-\mathrm{d} x^{2}+\mathrm{d} y^{2}+(\mathrm{d} z-x \mathrm{~d} y)^{2}
$$

$g$ é geodesicamente completa e seu grupo de isometrias tem dimensão 4 . Neste caso, o grupo Nil é munido com uma $\mathrm{SO}(2)$-estrutura, determinada pela escolha de um campo de Killing $E_{1}$. Obtemos, assim, um teorema de imersão isométrica no grupo Nil, onde prescrevemos o fibrado normal, a conexão normal, a segunda forma fundamental, a projeção do campo $E_{1}$ sobre $T M$ e a componente normal de $E_{1}$ (cf. Teorema 5.2.7). 
O grupo de Lie Sol (cf. Exemplo 1.4.9) é munido de uma métrica Lorentziana invariante à esquerda $g$, cujo grupo de isometrias tem dimensão 4 . Além disso, Sol é identificado com um espaço homogêneo $K / H$, onde $K$ é um grupo de Lie, $H$ é um subgrupo fechado de $K$ e a métrica $g$ é Sol-invariante (cf. Teorema 5.4.1). Neste caso, o grupo Sol, identificado com o quociente $K / H$, é munido de uma $\overline{\operatorname{Ad}}(H)$-estrutura; obtemos, assim, um teorema de imersão isométrica no grupo de Lie Sol.

Outro problema considerado em Geometria Diferencial é a questão de rigidez isométrica. Dizemos que duas imersões isométricas $f: M \rightarrow \bar{M}$ e $g: M \rightarrow \bar{M}$ são congruentes se existe uma isometria $\sigma: \bar{M} \rightarrow \bar{M}$ tal que $g=\sigma \circ f$. Uma imersão isométrica $f: M \rightarrow \bar{M}$ é dita ser rígida se $f$ é congruente com qualquer outra imersão isométrica $g: M \rightarrow \bar{M}$. Um resultado importante é um teorema de Allendoerfer [1], para o caso em que o ambiente é uma variedade Riemanniana de curvatura seccional constante $Q_{c}^{n+k}$. Mais precisamente, toda imersão isométrica $f: M^{n} \rightarrow Q_{c}^{n+k}$, com $n \geq 3$, cujo número tipo $\tau_{f}$ satisfaz $\tau_{f}(x) \geq 3$, para todo $x \in M$, é rígida. Abordagens desse problema, com essas hipóteses, podem ser encontradas em [7], [18], [29]. Outros resultados podem ser obtidos quando a subvariedade satisfaz condições mais fortes, como compacidade, completude, curvatura média constante, fibrado normal flat, dentre outras (ver, por exemplo, [2], [5], [8]).

Em nosso trabalho provamos também um teorema semelhante ao Teorema de Allendoerfer [1] para o caso de imersões isométricas que preservam $G$-estrutura. Mais precisamente, para o caso de hipersuperfícies de variedades semi-Riemannianas infinitesimalmente homogêneas com $G$-estrutura fixada (cf. Teorema 6.3.5). No caso particular em que o ambiente são variedades semi-Riemannianas da forma $Q_{c}^{n} \times \mathbb{R}$ ou variedades Riemannianas homogêneas tridimensionais, provamos o mesmo teorema de rigidez, porém com hipóteses mais fracas (cf. Teorema 6.4.1 e Teorema 6.4.2).

Finalizamos esta introdução com uma breve descrição do conteúdo de cada capítulo. Os Capítulos 1 e 2 foram incluídos para tornar a leitura tão auto-suficiente quanto possível. Embora o conteúdo de tais capítulos seja um assunto clássico na literatura, julgamos importante desenvolver um texto auto-contido, o que justifica um pouco a inclusão de tais capítulos.

O Capítulo 1, por ser bem elementar, está praticamente ausente de demonstrações. Porém, tomamos o cuidado de indicar ao leitor referências onde estas podem ser encontradas. Na Seção 1.1 apresentamos algumas definições básicas da teoria de variedades diferenciáveis. Nas Seções 1.2 e 1.3 falamos de álgebras de Lie e grupos de Lie. Enfatizamos as Subseções 1.3.1, 
1.3.2 e 1.3.3, onde discutimos produto semi-direto de grupos de Lie, grupos de Lie nilpotentes e grupos de Lie solúveis, respectivamente. As definições e resultados aí discutidos são usados naturalmente no Capítulo 5. Na Seção 1.4 listamos os grupos de Lie clássicos que serão usados no texto, bem como suas respectivas álgebras de Lie. Nas Seções 1.5 e 1.6 falamos de espaços homogêneos da forma $K / H$, onde $K$ é um grupo de Lie e $H$ é um subgrupo fechado de $K$. O resultado principal é o Lema 1.6.7, que estabelece uma correspondência entre métricas $K$-invariantes em $K / H$ e os produtos escalares $\overline{\operatorname{Ad}}(H)$-invariantes em $\mathfrak{k} / \mathfrak{g}$.

O Capítulo 2 é dedicado ao estudo dos espaços fibrados e conexões. Embora este também seja um capítulo elementar, procuramos manter a exposição essencialmente auto-contida. Uma exceção é feita a alguns resultados das Seções 2.4 e 2.5 (a demonstração do Teorema 2.4.10, por exemplo, passa pela construção do fibrado associado e nosso objetivo aqui não se estende a tais construções) que são enunciados sem demonstração; estas podem ser encontradas em livros de Geometria Diferencial, como [17] e [18]. Na Seção 2.1 falamos de fibrado principal, onde nos detemos apenas nas definições principais e alguns exemplos. Na Subseção 2.1.1 falamos de subfibrados principais; a Subseção 2.1.2 é dedicada aos morfismos de fibrados principais e na Subseção 2.1.3 falamos de pull-back de fibrados principais. Os fibrados vetoriais são estudados na Seção 2.2, onde listamos alguns exemplos que são utilizados no texto. As Subseções 2.2.1, 2.2.2, 2.2 .3 e 2.2.4 contemplam o estudo dos invariantes dos fibrados vetoriais, como seções, morfismos, pull-back e subfibrados vetoriais.

A Seção 2.3 é dedicada ao estudo de conexões em fibrados principais; enfatizamos aqui a correspondência entre conexões em fibrados principais e formas de conexão. Na Seção 2.4 falamos de conexões em fibrados vetoriais, onde definimos o tensor de Christoffel de uma conexão relativo a uma seção local fixada. Enfatizamos uma correspondência entre conexões em fibrados vetoriais e conexões em fibrados principais. Na Subseção 2.4.1 falamos de conexão em fibrado vetorial quociente, onde definimos a segunda forma fundamental de um subfibrado vetorial. Ela é essencialmente útil para o cálculo da inner torsion de alguns exemplos de $G$-estruturas (cf. Seção 3.3). Na Seção 2.5 estudamos a forma de curvatura; o objetivo é apenas fixar a linguagem e explicitar a relação existente entre o tensor de curvatura de uma conexão em um fibrado vetorial e a forma de conexão correspondente. Esta relação é utilizada na Seção 2.6, onde estudamos as conexões $K$-invariantes em espaços homogêneos $K / H$. O resultado principal é o Lema 2.6.2, que estabelece uma correspondência entre conexões $K$-invariantes em $K / H$ e aplicações lineares $\lambda: \mathfrak{m} \rightarrow \mathfrak{g l}(\mathfrak{m})$, onde $\mathfrak{m}$ é um subespaço de $\mathfrak{k}, \operatorname{com} \mathfrak{k}=\mathfrak{h} \oplus \mathfrak{m}$. A 
Subseção 2.6.1 contempla o caso particular em que a conexão $K$-invariante é a conexão de Levi-Civita de alguma métrica semi-Riemanniana $K$-invariante em $K / H$; neste caso, obtemos uma expressão simples para a aplicação $\lambda$ (cf. Teorema 2.6.7).

O Capítulo 3 é dedicado ao estudo de $G$-estruturas e da inner torsion. $\mathrm{Na}$ Seção 3.2 definimos $G$-estrutura em fibrados vetoriais e listamos alguns exemplos de $G$-estruturas, que são utilizados no texto. A Seção 3.3 é dedicada ao estudo da inner torsion de uma $G$-estrutura. A inner torsion de uma $G$-estrutura foi definida em [26], e pode ser vista como um tipo de derivada covariante da $G$-estrutura dada. Enfatizamos o Lema 3.3.7, que proporciona um método simples para o cálculo da inner torsion em termos do tensor de Christoffel. Na Seção 3.4 apresentamos o cálculo da inner torsion de $G$ estruturas em espaços homogêneos em termos da aplicação linear $\lambda$, dada pelo Lema 2.6.2.

No Capítulo 4 estudamos a teoria básica de imersões isométricas. Na Seção 4.2 estudamos as componentes de uma conexão; mais precisamente, a expressão da conexão em um fibrado vetorial que é dado como soma direta de dois subfibrados vetoriais. Na Seção 4.3 revemos as equações fundamentais de uma imersão isométrica, ou seja, as equações de Gauss, Codazzi e Ricci, analisando os casos de curvatura seccional constante e o caso de codimensão 1. A Seção 4.4 é dedicada ao estudo das variedades infinitesimalmente homogêneas (cf. Definição 4.4.1). Discutimos também o conceito de $G$-homogeneidade que, a menos de hipóteses topológicas, é equivalente ao conceito de homogeneidade infinitesimal (cf. Lema 4.4.6 e Lema 4.4.7). Listamos também vários exemplos de tais variedades que são usadas no texto. A Seção 4.5 é dedicada ao estudo do teorema (cf. Teorema 4.5.2) que garante existência de imersões isométricas que preservam $G$-estrutura em variedades semi-Riemannianas munidas de $G$-estrutura. Este teorema foi provado em [26] em um contexto mais geral, mais precisamente no contexto de imersões afins (ver [26, Teorema 7.4]). Apresentamos também alguns exemplos de aplicações do Teorema 4.5.2.

O Capítulo 5 é dedicado ao estudo de imersões isométricas em variedades Lorentzianas tridimensionais. A Seção 5.1 é de carácter inteiramente motivador. Falamos rapidamente do Teorema de Classificação de Thurston e do Teorema de Classificação Lorentziana, do qual estamos interessados. O caso Lorentziano foi provado recentemente (ver [11]), onde os autores mostram que existem somente quatro geometrias Lorentzianas maximais, a saber: Minkowski, anti-de Sitter, Heisenberg-Lorentz e Sol-Lorentz. A idéia central é usar a teoria desenvolvida em [26], interpretando as condições suficientes para existência de imersões isométricas, e provar um teorema de 
imersão isométrica em tais espaços. A Seção 5.2 é dedicada ao grupo de Heisenberg Nil. Na Subseção 5.2.1 estudamos alguns preliminares de Nil, explicitando seu tensor de curvatura. Na Subseção 5.2.2 estudamos as equações de compatibilidade para superfícies em Nil e, finalmente, na Subseção 5.2.3, provamos um teorema de imersão isométrica em Nil. Mais precisamente, fixado um campo de Killing $E_{1} \in \boldsymbol{\Gamma}(T \mathrm{Nil})$, provamos o seguinte:

Teorema 1 Sejam $(M, g)$ uma variedade Riemanniana de dimensão $2, \nabla$ sua conexão de Levi-Civita, $E=T M \times \mathbb{R}$ o fibrado vetorial trivial sobre $M$ munido de uma métrica semi-Riemanniana $g^{E}, \nabla^{E}$ uma conexão em $E$ $g^{E}$-compativel, a uma seção diferenciável simétrica de $\operatorname{Lin}_{2}(T M ; E)$. Denotemos por $\widehat{g}$ a métrica semi-Riemanniana no fibrado $\widehat{E}=T M \oplus E$ dada pela soma direta ortogonal de $g$ e $g^{E}$, e consideremos a conexão $\widehat{\nabla}$ em $\widehat{E}$ cujas componentes são $\nabla, \nabla^{E}$ e $\alpha$. Sejam $T \in \boldsymbol{\Gamma}(T M)$ e $\nu \in C^{\infty}(M)$ tais que $\|T\|^{2}+\nu^{2}=1$. Suponha que a terna $(M, \alpha,(T, \nu))$ satisfaça as equações de compatibilidade da Definição 5.2.6. Então, para quaisquer $x_{\mathrm{o}} \in M e$ $y_{\mathrm{o}} \in \mathrm{Nil}$, existem uma vizinhança $U$ de $x_{\mathrm{o}}$ em $M$ e uma imersão isométrica $f: U \rightarrow$ Nil tal que o operador de Weingarten $A$ de $f$ em relação ao normal $N$ é dado por

$$
\mathrm{d} f \circ A \circ \mathrm{d} f^{-1}
$$

e tal que

$$
E_{1}=\mathrm{d} f(T)+\nu N
$$

Além disso, se $M$ é conexa e simplesmente conexa, existe uma imersão isométrica global $f: M \rightarrow$ Nil satisfazendo (1) e (2).

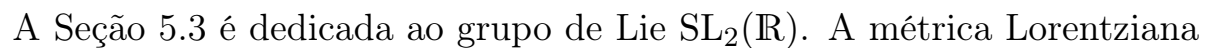
considerada em $\mathrm{SL}_{2}(\mathbb{R})$, bem como em $\mathbb{R}^{3}$, torna $\mathrm{SL}_{2}(\mathbb{R})$ uma variedade de curvatura seccional constante. Embora para esses espaços teoremas de imersão isométrica sejam bem conhecidos na literatura clássica, o teorema apresentado aqui é semelhante ao Teorema 1 e, portanto, diferente daquele apresentado na teoria clássica. Isso justifica um pouco esta seção. Na Subseção 5.3.1 estudamos alguns preliminares de $\mathrm{SL}_{2}(\mathbb{R})$; a Subseção 5.3.2 consiste das equações de compatibilidade para superfícies em $\mathrm{SL}_{2}(\mathbb{R})$ e na Subseção 5.3.3 provamos um teorema de imersão isométrica em $\mathrm{SL}_{2}(\mathbb{R})$, semelhante ao Teorema 1. Finalmente, na Seção 5.4 estudamos o grupo de Lie Sol. A Subseção 5.4.1 consiste de preliminares, onde enfatizamos o Teorema 5.4.1, que identifica o grupo Sol com um espaço homogêneo $K / H$. Provamos também que o grupo de isometrias de Sol tem dimensão igual a 4 e explicitamos seu 
tensor de curvatura. Na Subseção 5.4.2 provamos um teorema de imersão isométrica em Sol (cf. Teorema 5.4.5). O teorema é, ainda, um resultado abstrato, se comparado ao Teorema 1.

Finalmente, no Capítulo 6 estudamos o problema de rigidez isométrica para hipersuperfícies em variedades semi-Riemannianas munidas de $G$-estrutura. A Seção 6.1 é de carácter inteiramente introdutório, onde estabelecemos as definições e resultados clássicos. Na Seção ?? definimos os conceitos de $G$-congruência e $G$-rigidez isométrica para imersões isométricas que preservam $G$-estrutura. Provamos um resultado de $G$-congruência para o caso particular em que as imersões isométricas têm os mesmos dados prescritos (cf. Lema 6.2.3). Na Seção 6.2 provamos um teorema abstrato de $G$-rigidez para hipersuperfícies que preservam $G$-estrutura em variedades semi-Riemannianas munidas de $G$-estrutura. Mais precisamente, provamos o seguinte:

Teorema 2 Seja $(f, S)$ uma imersão isométrica de $M^{n}$ em $\bar{M}$ que preserva $G$-estrutura tal que $\operatorname{rank}\left(A_{x}\right) \geq 3$, para todo $x \in M$. Suponha, além disso, que $M$ é conexa e orientável, e $(\bar{M}, \bar{\nabla}, \bar{P})$ é globalmente homogênea. Então $(f, S)$ é G-rígida.

Na Seção 6.4 estudamos o problema de $G$-rigidez isométrica quando o espaço ambiente são variedades da forma $Q_{c}^{n} \times \mathbb{R}$ ou variedades Riemannianas homogêneas tridimensionais. Neste caso, a $G$-estrutura considerada em $\widehat{E}=T M \oplus E$ é caracterizada pela escolha de um campo $T \in \boldsymbol{\Gamma}(T M)$ e uma função $\nu \in \mathrm{C}^{\infty}(M)$ tais que $\|T\|^{2}+\nu^{2}=1$. Assim, para estes ambientes, provamos um teorema de rigidez com hipóteses mais fracas. Mais precisamente, denotando por $\bar{M}$ uma variedade da forma $Q_{c}^{n} \times \mathbb{R}$ ou uma variedade Riemanniana homogênea tridimensional, temos:

Teorema 3 Seja $(f, S)$ uma imersão isométrica que preserva $G$-estrutura de $M$ em $\bar{M}$. Se o conjunto $\{x \in M: \nu(x) \neq 0\}$ é denso em $M$, então $(f, S)$ é G-rígida. 


\section{Capítulo 1}

\section{Variedades e Grupos de Lie}

\subsection{Variedades diferenciáveis}

Uma carta local em um conjunto arbitrário $M$ é uma aplicação bijetora $\varphi: U \rightarrow \varphi(U)$, onde $U$ é um subconjunto arbitrário de $M$ e $\varphi(U)$ é um subconjunto aberto de algum espaço Euclidiano $\mathbb{R}^{n}$. Dados duas cartas locais $\varphi: U \rightarrow \varphi(U)$ e $\psi: V \rightarrow \psi(V)$ em $M$, a aplicação de transição de $\varphi$ para $\psi$ é a aplicação bijetora

$$
\psi \circ \varphi^{-1}: \varphi(U \cap V) \rightarrow \psi(U \cap V) .
$$

Dizemos que $\varphi$ e $\psi$ são compatíveis se $\varphi(U \cap V)$ e $\psi(U \cap V)$ são ambos abertos em $\mathbb{R}^{n}$ e a aplicação de transição (1.1) é um difeomorfismo diferenciável. Note que $\varphi$ e $\psi$ são compatíveis quando $U \cap V=\emptyset$. Um atlas no conjunto $M$ é um conjunto $\mathcal{A}$ de cartas locais em $M$, duas a duas compatíveis, cujos domínios formam uma cobertura do conjunto $M$.

Se duas cartas locais $\varphi$ e $\psi$ em $M$ são compatíveis com todas as cartas locais que pertencem a um atlas $\mathcal{A}$ em $M$, então $\varphi$ e $\psi$ são compatíveis. Assim, todo atlas $\mathcal{A}$ em $M$ está contido em um único atlas maximal, que consiste de todas as cartas locais em $M$ que são compatíveis com todas as cartas locais de $\mathcal{A}$. Um atlas maximal em um conjunto $M$ é chamado uma estrutura diferenciável em $M$.

Um atlas $\mathcal{A}$ em um conjunto $M$ induz uma única topologia $\tau$ em $M$ tal que, para toda carta local $\varphi: U \rightarrow \varphi(U)$ que pertence a $\mathcal{A}$, o conjunto $U$ é aberto em $(M, \tau)$ e a aplicação $\varphi$ é um homeomorfismo. Tal topologia consiste de todos os subconjuntos $A$ de $M$ tais que $\varphi(U \cap A)$ é aberto em $\mathbb{R}^{n}$, para toda carta local $\varphi: U \rightarrow \varphi(U)$ que pertence a $\mathcal{A}$. A topologia $\tau$ 
será chamada a topologia induzida pelo atlas $\mathcal{A}$. Se $\mathcal{A}$ e $\mathcal{A}^{\prime}$ são atlas em $M$, com $\mathcal{A} \subset \mathcal{A}^{\prime}$, então $\mathcal{A}$ e $\mathcal{A}^{\prime}$ induzem a mesma topologia em $M$.

Definição 1.1.1 Uma variedade diferenciável é um par $(M, \mathcal{A})$, onde $M$ é um conjunto e $\mathcal{A}$ é um atlas maximal em $M$ cuja topologia induzida $\tau$ por $\mathcal{A}$ é Hausdorff e satisfaz o segundo axioma da enumerabilidade.

Denotaremos simplesmente por $M^{n}$ uma variedade diferenciável, subentendendo o atlas maximal $\mathcal{A}$ dado na Definição 1.1.1. Assim, o termo carta local em $M$ passa a significar uma carta local que pertence a $\mathcal{A}$.

O Lema seguinte, que será útil ao estudarmos os espaços fibrados, permite-nos transportar a estrutura diferenciável de uma variedade diferenciável $M$ para um conjunto $N$ quando temos uma família de bijeções em correspondência. Mais precisamente, temos:

Lema 1.1.2 Sejam $M$ um conjunto e $\left\{\varphi_{i}\right\}_{i \in I}$ uma família de bijeções definidas em subconjuntos $U_{i} \subset M$ e tomando valores em variedades diferenciáveis $N_{i}$. Assuma que $M=\bigcup_{i \in I} U_{i}$ e que $\varphi_{i}$ e $\varphi_{j}$ são compativeis para quaisquer $i, j \in I$, ou seja, $\varphi_{i}\left(U_{i} \cap U_{j}\right)$ é aberto em $N_{i}, \varphi_{j}\left(U_{i} \cap U_{j}\right)$ é aberto em $N_{j}$ e a aplicação de transição

$$
\varphi_{j} \circ \varphi_{i}^{-1}: \varphi_{i}\left(U_{i} \cap U_{j}\right) \rightarrow \varphi_{j}\left(U_{i} \cap U_{j}\right)
$$

é diferenciável. Então, existe uma única estrutura diferenciável no conjunto $M$ tal que, para todo $i \in I$, o conjunto $U_{i}$ é aberto em $M$ e $\varphi_{i}: U_{i} \rightarrow N_{i}$ é um difeomorfismo diferenciável.

Demonstração. A idéia da demonstração consiste no seguinte. Para cada $i \in I$, consideremos

$$
\mathcal{A}_{i}=\left\{\psi_{i j}: V_{i j} \rightarrow \psi_{i j}\left(V_{i j}\right) \subset \mathbb{R}^{n}\right\}_{j \in I_{i}}
$$

uma estrutura diferenciável na variedade diferenciável $N_{i}$. Um cálculo simples mostra que o conjunto

$$
\mathcal{A}_{0}=\left\{\phi_{i j}=\left.\psi_{i j} \circ \varphi_{i}\right|_{\varphi_{i}^{-1}\left(V_{i j}\right)}: \varphi_{i}^{-1}\left(V_{i j}\right) \rightarrow \psi_{i j}\left(V_{i j}\right)\right\}_{i \in I, j \in I_{i}}
$$

é um atlas no conjunto $M$. Então, o único atlas maximal $\mathcal{A}$ que contém $\mathcal{A}_{0}$ é uma estrutura diferenciável em $M$ que satisfaz as propriedades exigidas. Para mostrar a unicidade da estrutura diferenciável, sejam $\mathcal{A}$ e $\mathcal{A}^{\prime}$ duas estruturas diferenciáveis em $M$ que tornam cada subconjunto $U_{i}$ aberto em 
$M$ e cada $\varphi_{i}$ um difeomorfismo diferenciável. Seja $\left.\mathcal{A}\right|_{U_{i}}\left(\right.$ resp., $\left.\left.\mathcal{A}^{\prime}\right|_{U_{i}}\right)$ a estrutura diferenciável induzida por $\mathcal{A}$ (resp., por $\mathcal{A}^{\prime}$ ) em $U_{i}$. Temos, então, o seguinte diagrama comutativo:

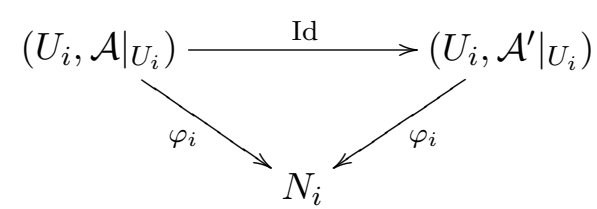

onde ambas as flechas $\varphi_{i}$ são difeomorfismos diferenciáveis. Isso mostra que a aplicação identidade $\operatorname{Id}:(M, \mathcal{A}) \rightarrow\left(M, \mathcal{A}^{\prime}\right)$, restrita ao aberto $U_{i}$, é um difeomorfismo diferenciável. Como $M=\bigcup_{i \in I} U_{i}$, segue que Id é um difeomorfismo diferenciável e, portanto, $\mathcal{A}=\mathcal{A}^{\prime}$.

Um subconjunto $N$ de uma variedade diferenciável $M^{n}$ é chamado uma subvariedade mergulhada de $M$ se, para todo $x \in N$, existe uma carta local $\varphi: U \rightarrow \varphi(U)$ de $M$, com $x \in U$, satisfazendo $\varphi(U \cap N)=\varphi(U) \cap \mathbb{R}^{k}$ para um mesmo espaço Euclidiano $\mathbb{R}^{k}, 0 \leq k \leq n$. A carta local $\varphi: U \rightarrow \varphi(U)$ é chamada uma carta de subvariedade para $N$. Diremos também que

$$
\left.\varphi\right|_{U \cap N}: U \cap N \rightarrow \varphi(U) \cap \mathbb{R}^{k}
$$

é a carta induzida por $\varphi$ em $N$.

As cartas induzidas (1.2) em $N$ determinam um atlas em $N$; a topologia de $N$ será então induzida da topologia de $M$. A aplicação inclusão i : $N \rightarrow M$ será, neste caso, um mergulho, i.e., uma imersão diferenciável que é também um homeomorfismo sobre sua imagem (com a topologia induzida do contradomínio).

Uma subvariedade imersa de $N$ em $M$ é uma variedade diferenciável $N$ tal que $N \subset M$ como conjunto de modo que a aplicação inclusão i $: N \rightarrow M$ seja uma imersão diferenciável. Observe que um mesmo subconjunto $N \subset M$ pode ter várias estruturas diferenciáveis que o tornam uma subvariedade imersa de $M$. No entanto, fixada uma topologia em $N$, existe no máximo uma estrutura diferenciável compatível com essa topologia que torna $N$ uma subvariedade imersa de $M$ (ver Lema 1.1.3).

Se $N$ e $M$ são variedades diferenciáveis arbitrárias e $f: N \rightarrow M$ é uma imersão diferenciável injetora então existe uma única estrutura diferenciável em $f(N)$ que torna $f: N \rightarrow f(N)$ um difeomorfismo diferenciável. Segue então que $f(N)$ é uma subvariedade imersa de $M$. Se $f$ for um mergulho então $f(N)$ será uma subvariedade mergulhada de $M$. 
Lema 1.1.3 Sejam $M, N$ variedades diferenciáveis, $P \subset N$ uma subvariedade mergulhada e $f: M \rightarrow N$ uma aplicação diferenciável, com $f(M) \subset P$. Então, existe uma única aplicação diferenciável $f_{0}: M \rightarrow P$ que torna o diagrama

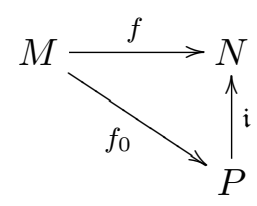

comutativo, onde $\mathfrak{i}: P \rightarrow N$ denota a aplicação inclusão.

Demonstração. Ver [36, Teorema 1.32].

Dizemos que a aplicação $f_{0}$, dada no Lema 1.1.3, é obtida de $f$ por mudança de contra-domínio.

Observação 1.1.4 O Lema 1.1.3 não vale, em geral, se $N$ é apenas uma subvariedade imersa de $M$. Porém, mesmo nesse caso, podemos concluir que $f_{0}$ é diferenciável ser supormos que $f_{0}$ seja contínua (ver [36, Teorema 1.32]).

Observação 1.1.5 Subvariedades imersas $N \subset M$ para as quais a diferenciabilidade de $f$ implica na diferenciabilidade de $f_{0}$ em (1.3) são conhecidas como subvariedades quase-mergulhadas. Exemplos de tais subvariedades são as subvariedades integrais de distribuições involutivas (ver [36, Teorema 1.62]) e as subvariedades imersas que são também subgrupos abstratos de um grupo de Lie (ver [36, Teorema 3.20 e Corolário (b) do Teorema 3.19]).

Proposição 1.1.6 Sejam $M, N$ variedades diferenciáveis e $f: M \rightarrow N$ uma aplicação. Então, $f$ é um mergulho diferenciável se, e somente se, para todo $x \in M$, existe uma vizinhança $U$ de $f(x)$ em $N$ tal que $f^{-1}(U)$ é aberto em $M$ e $\left.f\right|_{f^{-1}(U)}: f^{-1}(U) \rightarrow N$ é um mergulho diferenciável.

Demonstração. Se $f$ é um mergulho diferenciável, basta tomar $U=$ $f(M)$, para cada $x \in U$. Reciprocamente, dado $x \in M$, existe, por hipótese, uma vizinhança $U$ de $f(x)$ em $N$ tal que $f^{-1}(U)$ é aberto em $M$ e $\left.f\right|_{f^{-1}(U)}$ : $f^{-1}(U) \rightarrow N$ é um mergulho diferenciável. Disso decorre que $f: M \rightarrow N$ é uma imersão diferenciável. Resta mostrar apenas que $f: M \rightarrow f(M)$ é aberta. Mas isso segue do fato de que $f: M \rightarrow f(M)$ é, localmente, uma aplicação aberta. 
Observação 1.1.7 Se $f: M \rightarrow N$ é uma submersão diferenciável, segue da forma local das submersões que, para todo $x \in M$, existem uma vizinhança $U$ de $f(x)$ em $N$ e uma aplicação diferenciável $s: U \rightarrow M$ tal que $f \circ s$ é a aplicação de inclusão. A aplicação $s$ é chamada uma seção local diferenciável de $f$.

\section{2 Álgebras de Lie}

Um espaço vetorial $\mathfrak{g}$ sobre um corpo $\mathbb{K}$ de característica 0 é chamado uma álgebra de Lie sobre $\mathbb{K}$ se existe uma aplicação bilinear anti-simétrica

$$
(X, Y) \in \mathfrak{g} \times \mathfrak{g} \longmapsto[X, Y] \in \mathfrak{g}
$$

que satisfaz a seguinte propriedade:

$$
[X,[Y, Z]]+[Y,[Z, X]]+[Z,[X, Y]]=0,
$$

para quaisquer $X, Y, Z \in \mathfrak{g}$. Dados $X, Y \in \mathfrak{g}$, o elemento $[X, Y] \in \mathfrak{g}$ é chamado o colchete de Lie de $X$ e $Y$. A relação (1.4) é conhecida como a identidade de Jacobi.

Seja $\mathfrak{g}$ uma álgebra de Lie sobre $\mathbb{K}$. Dados dois subespaços vetoriais $\mathfrak{a}, \mathfrak{b}$ de $\mathfrak{g}$, denotemos por $[\mathfrak{a}, \mathfrak{b}]$ o subespaço gerado por $[X, Y]$, com $X \in \mathfrak{a}$ e $Y \in \mathfrak{b}$. Um subespaço vetorial $\mathfrak{h}$ de $\mathfrak{g}$ é chamado uma subálgebra de $\mathfrak{g}$ se $[\mathfrak{h}, \mathfrak{h}] \subseteq \mathfrak{h}$; é chamado um ideal se $[\mathfrak{g}, \mathfrak{h}] \subseteq \mathfrak{h}$.

Sejam $\mathfrak{g}, \mathfrak{h}$ álgebras de Lie sobre $\mathbb{K}$. Uma aplicação linear $\sigma: \mathfrak{g} \rightarrow \mathfrak{h}$ é chamada um homomorfismo de álgebras de Lie se $\sigma$ preserva a operação de colchete, ou seja, se

$$
[\sigma(X), \sigma(Y)]=\sigma([X, Y]),
$$

para quaisquer $X, Y \in \mathfrak{g}$. Um homomorfismo de álgebras de Lie $\sigma: \mathfrak{g} \rightarrow \mathfrak{h}$ que também é um isomorfismo (de espaços vetoriais) é chamado um isomorfismo de álgebras de Lie. Quando $\mathfrak{h}=\mathfrak{g}$, um isomorfismo de álgebras de Lie $\sigma: \mathfrak{g} \rightarrow \mathfrak{g}$ é chamado um automorfismo de $\mathfrak{g}$. Se $\sigma: \mathfrak{g} \rightarrow \mathfrak{h}$ é um homomorfismo de álgebras de Lie, então $\sigma(\mathfrak{g})$ é uma subálgebra de $\mathfrak{h}$ e o núcleo de $\sigma$ é um ideal em $\mathfrak{g}$.

Reciprocamente, seja $\mathfrak{g}$ uma álgebra de Lie sobre $\mathbb{K}$ e $\mathfrak{h}$ um ideal de $\mathfrak{g}$. Sejam $\mathfrak{g} / \mathfrak{h}$ o espaço vetorial quociente e $\pi: \mathfrak{g} \rightarrow \mathfrak{g} / \mathfrak{h}$ a aplicação linear quociente. Dados $\bar{X}=\pi(X)$ e $\bar{Y}=\pi(Y)$, defina:

$$
[\bar{X}, \bar{Y}]=\pi([X, Y])
$$


para quaisquer $X, Y \in \mathfrak{g}$. Um cálculo simples mostra que (1.6) está bem definido e torna $\mathfrak{g} / \mathfrak{h}$ uma álgebra de Lie sobre $\mathbb{K}$ com esta definição de colchete. Assim, $\pi$ é um homomorfismo de álgebras de Lie, cujo núcleo é $\mathfrak{h}$; $\mathfrak{g} / \mathfrak{h}$ é chamado o quociente de $\mathfrak{g}$ por $\mathfrak{h}$.

Exemplo 1.2.1 Sejam $\mathfrak{g}_{1}, \ldots, \mathfrak{g}_{n}$ álgebras de Lie sobre $\mathbb{K}$. Então,

$$
\mathfrak{g}=\mathfrak{g}_{1} \times \cdots \times \mathfrak{g}_{n}
$$

torna-se uma álgebra de Lie sobre $\mathbb{K}$ com a operação:

$$
\left[\left(X_{1}, \ldots, X_{n}\right),\left(Y_{1}, \ldots, Y_{n}\right)\right]=\left(\left[X_{1}, Y_{1}\right], \ldots,\left[X_{n}, Y_{n}\right]\right),
$$

para quaisquer $X_{i}, Y_{i} \in \mathfrak{g}_{i}, 1 \leq i \leq n ; \mathfrak{g}$ é chamada o produto das álgebras de Lie $\mathfrak{g}_{i}, 1 \leq i \leq n$.

Exemplo 1.2.2 Seja $\mathfrak{g}$ um espaço vetorial sobre $\mathbb{K}$ de dimensão finita. Definindo $[X, Y]=0$, para quaisquer $X, Y \in \mathfrak{g}$, $\mathfrak{g}$ torna-se uma álgebra de Lie sobre $\mathbb{K}$. Uma álgebra de Lie $\mathfrak{g}$ sobre $\mathbb{K}$ que satisfaz $[\mathfrak{g}, \mathfrak{g}]=\{0\}$ é chamada abeliana.

Exemplo 1.2.3 Seja $V$ um espaço vetorial sobre $\mathbb{K}$ de dimensão finita e consideremos $\operatorname{Lin}(V)$ o espaço vetorial dos endomorfismos de $V$. Dados $X, Y \in \operatorname{Lin}(V)$, definimos

$$
[X, Y]=X Y-Y X .
$$

Com esta operação de colchete, o espaço vetorial $\operatorname{Lin}(V)$ torna-se uma álgebra de Lie sobre $\mathbb{K}$, usualmente denotada por $\mathfrak{g l}(V)$. Mais precisamente, o espaço vetorial das matrizes $n \times n$ sobre $\mathbb{K}$ torna-se uma álgebra de Lie sobre $\mathbb{K}$ definindo-se a operação de colchete por (1.7).

Exemplo 1.2.4 Seja $\mathfrak{g}$ uma álgebra de Lie sobre $\mathbb{K}$. Um endomorfismo $D: \mathfrak{g} \rightarrow \mathfrak{g}$ é chamado uma derivação de $\mathfrak{g}$ se

$$
D([X, Y])=[D(X), Y]+[X, D(Y)],
$$

para quaisquer $X, Y \in \mathfrak{g}$. Se $D_{1}$ e $D_{2}$ são derivações de $\mathfrak{g}$ então

$$
\left[D_{1}, D_{2}\right]=D_{1} D_{2}-D_{2} D_{1}
$$

é também uma derivação de $\mathfrak{g}$. Se $\mathfrak{g}$ tem dimensão finita então o conjunto das derivações de $\mathfrak{g}$ é uma subálgebra de $\mathfrak{g l}(\mathfrak{g})$. 
Exemplo 1.2.5 Dado uma variedade diferenciável $M$ de dimensão $n$, denotemos por $\boldsymbol{\Gamma}(T M)$ o espaço vetorial dos campos vetoriais diferenciáveis de $M$. O conjunto $\boldsymbol{\Gamma}(T M)$, munido do colchete de Lie de campos vetoriais, é uma álgebra de Lie sobre $\mathbb{R}$, em geral de dimensão infinita.

Sejam $\mathfrak{g}$ uma álgebra de Lie sobre $\mathbb{K}$ e $V$ um espaço vetorial sobre $\mathbb{K}$, não necessariamente de dimensão finita. Uma representação de $\mathfrak{g}$ em $V$ é uma aplicação linear $\sigma: \mathfrak{g} \rightarrow \operatorname{Lin}(V)$ que satisfaz:

$$
\sigma([X, Y])=\sigma(X) \sigma(Y)-\sigma(Y) \sigma(X),
$$

para quaisquer $X, Y \in \mathfrak{g}$. Se $V$ tem dimensão finita, a condição (1.9) é equivalente a dizer que $\sigma$ é um homomorfismo de álgebras de Lie de $\mathfrak{g}$ sobre $\mathfrak{g l}(V)$.

Exemplo 1.2.6 Seja $\mathfrak{g}$ uma álgebra de Lie sobre $\mathbb{K}$. Dado $X \in \mathfrak{g}$, denotemos por

$$
\operatorname{ad}_{X}: \mathfrak{g} \rightarrow \mathfrak{g}
$$

o endomorfismo de $\mathfrak{g}$ definido por

$$
\operatorname{ad}_{X}(Y)=[X, Y]
$$

para todo $Y \in \mathfrak{g}$. Um cálculo simples mostra que $\operatorname{ad}_{X}$ é uma derivação de $\mathfrak{g}$ e a aplicação

$$
X \in \mathfrak{g} \longmapsto \operatorname{ad}_{X} \in \operatorname{Lin}(\mathfrak{g})
$$

é uma representação de $\mathfrak{g}$ em $\mathfrak{g}$, chamada a representação adjunta de $\mathfrak{g}$. Note que $\mathfrak{g}$ é abeliana se, e somente se, $\operatorname{ad}_{X}=0$, para todo $X \in \mathfrak{g}$. O núcleo da representação adjunta, chamado o centro de $\mathfrak{g}$, é o conjunto de todos os $X \in \mathfrak{g}$ tais que $[X, Y]=0$, para todo $Y \in \mathfrak{g}$.

Exemplo 1.2.7 Sejam $\mathfrak{g}, \mathfrak{h}$ álgebras de Lie sobre $\mathbb{K}$ e $\sigma: \mathfrak{h} \rightarrow \operatorname{Lin}(\mathfrak{g})$ uma representação de $\mathfrak{h}$ em $\mathfrak{g}$ tal que $\sigma(Y)$ é uma derivação de $\mathfrak{g}$ para todo $Y \in \mathfrak{h}$. Dados $X, X^{\prime} \in \mathfrak{g}$ e $Y, Y^{\prime} \in \mathfrak{h}$, definimos:

$$
\left[(X, Y),\left(X^{\prime}, Y^{\prime}\right)\right]=\left(\left[X, X^{\prime}\right]+\sigma(Y) X^{\prime}-\sigma\left(Y^{\prime}\right) X,\left[Y, Y^{\prime}\right]\right) .
$$

O espaço vetorial $\mathfrak{g} \times \mathfrak{h}$, munido da operação (1.13), torna-se uma álgebra de Lie sobre $\mathbb{K}$, chamada o produto semi-direto de $\mathfrak{g} e \mathfrak{h}$ em relação à representação $\sigma$. Esta álgebra de Lie será denotada por $\mathfrak{h} \ltimes \mathfrak{g}$, subentendendo a representação $\sigma$. Se $\sigma=0$, obtemos o produto direto das álgebras de Lie $\mathfrak{g}$ e h, como no Exemplo 1.2.1. 
Definição 1.2.8 Um produto escalar $\langle\cdot, \cdot\rangle$ em um espaço vetorial $V$ é uma forma bilinear simétrica não-degenerada em $V$.

Definição 1.2.9 A forma de Killing de uma álgebra de Lie $\mathfrak{g}$ é a aplicação $\langle\cdot, \cdot\rangle: \mathfrak{g} \times \mathfrak{g} \rightarrow \mathbb{R}$ definida por

$$
\langle X, Y\rangle=\operatorname{tr}\left(\operatorname{ad}_{X} \circ \operatorname{ad}_{Y}\right)
$$

para quaisquer $X, Y \in \mathfrak{g}$.

Lema 1.2.10 A forma de Killing $\langle\cdot, \cdot\rangle$ de $\mathfrak{g}$ é uma forma bilinear simétrica que é invariante sob todos os automorfismos de $\mathfrak{g}$.

Demonstração. $\langle\cdot, \cdot\rangle$ é bilinear pois a aplicação $X \in \mathfrak{g} \longmapsto \operatorname{ad}_{X} \in \mathfrak{g l}(\mathfrak{g})$ é linear; é simétrica pois $\operatorname{tr}(A B)=\operatorname{tr}(B A)$. Seja $\sigma: \mathfrak{g} \rightarrow \mathfrak{g}$ um automorfismo de $\mathfrak{g}$. Por definição, temos $\sigma([X, Y])=[\sigma(X), \sigma(Y)]$, para quaisquer $X, Y \in \mathfrak{g}$, ou seja, $\sigma \circ \operatorname{ad}_{X}=\operatorname{ad}_{\sigma(X)} \circ \sigma$. Assim, $\operatorname{ad}_{\sigma(X)}=\sigma \circ \operatorname{ad}_{X} \circ \sigma^{-1}$. Como $\operatorname{tr}\left(A B A^{-1}\right)=\operatorname{tr}(B)$, segue que $\langle\sigma(X), \sigma(Y)\rangle=\langle X, Y\rangle$, para quaisquer $X, Y \in \mathfrak{g}$.

\subsection{Grupos de Lie}

Um grupo de Lie é uma variedade diferenciável $G$ munida de uma estrutura de grupo de modo que a aplicação

$$
(g, h) \in G \times G \longmapsto g h^{-1} \in G
$$

é diferenciável. O elemento identidade de $G$ será denotado por $1 \in G$. De (1.15) decorre que as translações à esquerda

$$
L_{g}: G \rightarrow G
$$

e as translações à direita

$$
R_{g}: G \rightarrow G
$$

definidas por $L_{g}(h)=g h$ e $R_{g}(h)=h g$, para todo $h \in G$, são difeomorfismos. O automorfismo interno de $G$ associado a $g$ é o difeomorfismo

$$
\mathcal{I}_{g}: G \rightarrow G
$$

definido por $\mathcal{I}_{g}(h)=g h g^{-1}$, para todo $h \in G$. 
Seja $G$ um grupo de Lie. Um campo vetorial $X$ (não necessariamente diferenciável) em $G$ é chamado invariante à esquerda se, para cada $g \in G$, $X$ é $L_{g}$-relacionado com $X$, ou seja, se

$$
\mathrm{d} L_{g}(h) \cdot X(h)=X(g h),
$$

para quaisquer $g, h \in G$. Para isso, basta que $\mathrm{d} L_{g}(1) \cdot X(1)=X(g)$, para todo $g \in G$. De fato, dado $h \in G$, temos:

$$
\begin{aligned}
\mathrm{d} L_{g}(h) \cdot X(h) & =\mathrm{d} L_{g}(h)\left(\mathrm{d} L_{h}(1) \cdot X(1)\right) \\
& =\mathrm{d}\left(L_{g} \circ L_{h}\right)(1) \cdot X(1)=\mathrm{d} L_{g h}(1) \cdot X(1) \\
& =X(g h) .
\end{aligned}
$$

Denotemos por $\mathfrak{g}$ o conjunto de todos os campos vetoriais invariantes à esquerda de $G$. Com a operação usual de soma de campos vetoriais, $\mathfrak{g}$ torna-se um subespaço vetorial de $\boldsymbol{\Gamma}(T G)$ e a aplicação

$$
X \in \mathfrak{g} \longmapsto X(1) \in T_{1} G
$$

é um isomorfismo linear (ver [36, Proposição 3.7]). Consequentemente,

$$
\operatorname{dim}(g)=\operatorname{dim}\left(T_{1} G\right)=\operatorname{dim}(G) .
$$

Além disso, campos vetoriais invariantes à esquerda são diferenciáveis, o colchete de Lie $[X, Y]$ pertence a $\mathfrak{g}$ se $X, Y \in \mathfrak{g}$, e $\mathfrak{g}$ torna-se uma álgebra de Lie sob a operação do colchete de Lie de campos vetoriais.

Dado um grupo de Lie $G$, definimos a álgebra de Lie de $G$ como sendo a álgebra de Lie $\mathfrak{g}$ dos campos vetoriais invariantes à esquerda em $G$. Alternativamente, podemos definir a álgebra de Lie de $G$ como o espaço tangente de $G$ no elemento identidade $1 \in G$, exigindo que o isomorfismo (1.20) seja um isomorfismo de álgebras de Lie.

Sejam $G, H$ grupos de Lie. Uma aplicação $\rho: G \rightarrow H$ é um homomorfismo de grupos de Lie se $\rho$ é um homomorfismo (de grupos abstratos) que é também contínuo; disso decorre que $\rho$ é automaticamente diferenciável (ver [36, Teorema 3.39]). Quando $H=\mathrm{GL}(V)$, para algum espaço vetorial real $V$ de dimensão finita, um homomorfismo $\rho: G \rightarrow \mathrm{GL}(V)$ é chamado uma representação de $G$ em $V$. Um homomorfismo $\rho: G \rightarrow H$ que é também um difeomorfismo é chamado um isomorfismo de grupos de Lie. Quando $G=H$, um isomorfismo $\rho: G \rightarrow G$ é chamado um automorfismo de grupos de Lie. 
Seja $\rho: G \rightarrow H$ um homomorfismo de grupos de Lie. Como $\rho\left(1_{G}\right)=1_{H}$, a identificação natural (1.20) faz com que a transformação linear $\mathrm{d} \rho\left(1_{G}\right)$ : $T_{1_{G}} G \rightarrow T_{1_{H}} H$ induza uma transformação linear de $\mathfrak{g}$ em $\mathfrak{h}$, que também será denotada por $\mathrm{d} \rho$. Assim, $\mathrm{d} \rho(X)$ é o único campo vetorial invariante à esquerda em $H$ tal que

$$
\mathrm{d} \rho(X)\left(1_{H}\right)=\mathrm{d} \rho\left(X\left(1_{G}\right)\right),
$$

para todo $X \in \mathfrak{g}$. Além disso, $\mathrm{d} \rho: \mathfrak{g} \rightarrow \mathfrak{h}$ é um homomorfismo de álgebras de Lie (ver [36, Teorema 3.14]).

Sejam $G$ um grupo de Lie e $\mathfrak{g}$ sua álgebra de Lie. Um subgrupo a 1parâmetro de $G$ é um homomorfismo $\rho: \mathbb{R} \rightarrow G$. Dado $X \in \mathfrak{g}$, a aplicação

$$
\lambda \frac{\mathrm{d}}{\mathrm{d} t} \longmapsto \lambda X
$$

é um homomorfismo da álgebra de Lie de $\mathbb{R}$ sobre $\mathfrak{g}$. Como $\mathbb{R}$ é simplesmente conexo, existe um único subgrupo a 1-parâmetro (ver [36, Teorema 3.27]) de $G$

$$
\exp _{X}: \mathbb{R} \rightarrow G
$$

tal que

$$
\operatorname{dexp}_{X}\left(\lambda \frac{\mathrm{d}}{\mathrm{d} t}\right)=\lambda X
$$

Ou seja, $t \in \mathbb{R} \longmapsto \exp _{X}(t) \in G$ é o único subgrupo a 1-parâmetro de $G$ tal que $\exp _{X}(0)=X(1)$. Definimos a aplicação exponencial de $G$

$$
\exp : \mathfrak{g} \rightarrow G
$$

por

$$
\exp (X)=\exp _{X}(1)
$$

para todo $X \in \mathfrak{g}$. A aplicação exponencial é diferenciável e dexp : $\mathfrak{g} \rightarrow$ $T_{1} G$ é a aplicação identidade (com a identificação (1.20)); assim exp é um difeomorfismo local de uma vizinhança de $0 \in \mathfrak{g}$ sobre uma vizinhança de $1 \in G$ (ver [36, Teorema 3.31]).

Seja $G$ um grupo de Lie. Um subgrupo de Lie de $G$ é uma subvariedade imersa $H \subset G$ que é também um subgrupo abstrato de $G$. Assim, $H$ tornase também um grupo de Lie com a operação de grupo induzida por $G$ (ver [36, Teorema 3.20]). Um subgrupo de Lie $H$ de $G$ é uma subvariedade mergulhada de $G$ se, e somente se, $H$ é fechado em $G$ (ver [36, Teorema 
3.21]). Além disso, todo subgrupo (abstrato) fechado $H \subset G$ é um subgrupo de Lie de $G$ (ver [36, Teorema 3.42]).

Se $H$ é um subgrupo de Lie de $G$ então a diferencial da aplicação inclusão $\mathfrak{i}: H \rightarrow G$ permite indentificar a álgebra de Lie $\mathfrak{h}$ de $H$ com uma subálgebra de g. Explicitamente, temos (ver [36, Proposição 3.33]):

$$
\mathfrak{h}=\{X \in \mathfrak{g}: \exp (t X) \in H, \forall t \in \mathbb{R}\} .
$$

Todo subgrupo discreto $H \subset G$ é um subgrupo de Lie mergulhado (e fechado) de $G, \operatorname{com} \operatorname{dim}(H)=0$. Neste caso, tem-se $\mathfrak{h}=\{0\}$.

Lema 1.3.1 Sejam $G$ um grupo de Lie, $V$ um espaço vetorial real de dimensão finita e $\rho: G \rightarrow \mathrm{GL}(V)$ uma representação de $G$ em $V$. Se $W$ é um subespaço vetorial de $V$ tal que $\rho(g)(W)=W$, para todo $g \in G$, então $\mathrm{d} \rho_{1}(X)(W) \subset W$, para todo $X \in \mathfrak{g}$. Reciprocamente, se $G$ é conexo e $\mathrm{d} \rho_{1}(X)(W) \subset W$, para todo $X \in \mathfrak{g}$, então $\rho(g)(W)=W$, para todo $g \in G$.

Demonstração. Suponha que $\rho(g)(W)=W$, para todo $g \in G$. Isso significa que $\rho(g) \in \mathrm{GL}(V ; W)$, para todo $g \in G$ (cf. notação no Exemplo 1.4.4). Assim, podemos considerar $\rho$ com contra-domínio $\mathrm{GL}(V ; W)$ (cf. Lema 1.1.3) e, portanto, a diferencial $\mathrm{d} \rho_{1}$ tem contra-domínio $\mathfrak{g l}(V ; W)$, i.e., $\mathrm{d} \rho_{1}(\mathfrak{g}) \subset \mathfrak{g l}(V ; W)$. Isso significa que $\mathrm{d} \rho_{1}(X)(W) \subset W$, para todo $X \in \mathfrak{g}$. Reciprocamente, suponha $\mathrm{d} \rho_{1}(X)(W) \subset W$, para todo $X \in \mathfrak{g}$, e consideremos o seguinte diagrama comutativo:

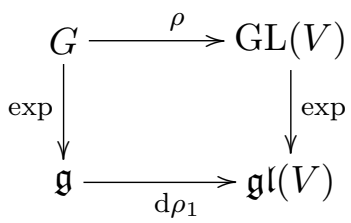

(ver [36, Teorema 3.32]). Como $G$ é conexo, $G$ é gerado por $\exp (\mathfrak{g})$. Assim, podemos supor, sem perda de generalidade, que $g \in G$ é da forma $g=$ $\exp (X), X \in \mathfrak{g}$. Como $\exp (\mathfrak{g l}(V ; W)) \subset \mathrm{GL}(V ; W)$, a comutatividade do diagrama (1.21) implica que $\rho(g) \in \mathrm{GL}(V ; W)$, para todo $g \in G$, concluindo a prova.

\subsubsection{Produto semi-direto de grupos de Lie}

Sejam $G, H$ grupos de Lie e $\rho: H \rightarrow \operatorname{Aut}(G)$ um homomorfismo de grupos de Lie. Para cada $h \in H$, denotemos por $\rho_{h} \in \operatorname{Aut}(G)$ a imagem de 
$h$ por $\rho$. Dados $g_{1}, g_{2} \in G$ e $h_{1}, h_{2} \in H$, definimos uma operação no produto $G \times H$ por:

$$
\left(g_{1}, h_{1}\right) \cdot\left(g_{2}, h_{2}\right)=\left(g_{1} \rho_{h_{1}}\left(g_{2}\right), h_{1} h_{2}\right) .
$$

O conjunto $G \times H$, munido da operação (1.22), torna-se um grupo de Lie, chamado o produto semi-direto de $G$ e $H$ em relação ao homomorfismo $\rho$, e será denotado por $H \ltimes G$, subentendendo o homomorfismo $\rho$.

O elemento identidade de $H \ltimes G$ é $1=\left(1_{G}, 1_{H}\right)$ e o elemento inverso de $(g, h) \in H \ltimes G$ é

$$
(g, h)^{-1}=\left(\rho_{h^{-1}}\left(g^{-1}\right), h^{-1}\right) .
$$

De (1.22) segue que $G$ é um subgrupo normal de $H \ltimes G$. Além disso, se todo $\rho_{h}$ é o automorfismo identidade, então $H \ltimes G$ é simplesmente o produto direto usual $G \times H$ de grupos de Lie.

Sejam $\mathfrak{g}, \mathfrak{h}$ as álgebras de Lie de $G$ e $H$, respectivamente. Para cada $h \in H$, a aplicação $\tau_{h}=\mathrm{d} \rho_{h}\left(1_{G}\right): \mathfrak{g} \rightarrow \mathfrak{g}$ é um automorfismo de $\mathfrak{g}$ que satisfaz:

$$
\rho_{h}(\exp (X))=\exp \left(\tau_{h}(X)\right),
$$

para todo $X \in \mathfrak{g}$. De (1.23) e do fato que $\rho: H \rightarrow \operatorname{Aut}(\mathrm{G})$ é um homomorfismo segue que

$$
\tau: H \rightarrow \mathrm{GL}(\mathfrak{g})
$$

definida por $\tau(h)=\tau_{h}$, para todo $h \in H$, é um homomorfismo de grupos de Lie. Denotemos por $\sigma$ a diferencial de $\tau$ no elemento identidade $1_{H} \in H$. Como cada $\tau_{h}$ é um automorfismo de $\mathfrak{g}$, segue que $\sigma(Y)$ é uma derivação de $\mathfrak{g}$, para todo $Y \in \mathfrak{h}$. Assim, podemos formar o produto semi-direto

$$
\mathfrak{h} \ltimes \mathfrak{g}
$$

(cf. Exemplo 1.2.7), chamado o produto semi-direto associado com $H \ltimes G$.

Lema 1.3.2 Existe um isomorfismo natural da álgebra de Lie de $H \ltimes G$ com a álgebra de Lie (1.24).

Demonstração. Dados $g \in G$ e $h \in H$, denotemos por

$$
g^{\prime}=\left(g, 1_{H}\right) \quad \text { e } \quad h^{\prime}=\left(1_{G}, h\right)
$$

e consideremos

$$
G^{\prime}=G \times\left\{1_{H}\right\} \quad \text { e } \quad H^{\prime}=\left\{1_{G}\right\} \times H .
$$


Para quaisquer $g_{1}, g_{2} \in G$ e $h_{1}, h_{2} \in H$ temos:

$$
\left(g_{1}, h_{1}\right)\left(g_{2}, h_{2}\right)\left(g_{1}, h_{1}\right)^{-1}=\left(g_{1} \rho_{h_{1}}\left(g_{2}\right) \rho_{h_{1} h_{2} h_{1}^{-1}}\left(g_{1}^{-1}\right), h_{1} h_{2} h_{1}^{-1}\right) .
$$

Isso implica que $G^{\prime}$ é um subgrupo normal fechado de $H \ltimes G, H^{\prime}$ é um subgrupo fechado de $H \ltimes G$ e

$$
h^{\prime} g^{\prime}\left(h^{\prime}\right)^{-1}=\rho_{h}(g)^{\prime} .
$$

Denotemos por $\mathfrak{a}$ a álgebra de Lie de $H \ltimes G$, e sejam $\mathfrak{g}^{\prime}, \mathfrak{h}^{\prime}$ as álgebras de Lie de $G^{\prime}$ e $H^{\prime}$, respectivamente; $\mathfrak{g}^{\prime}$ é um ideal de $\mathfrak{a}$ e

$$
\mathfrak{g}^{\prime}+\mathfrak{h}^{\prime}=\mathfrak{a} \quad \text { e } \quad \mathfrak{g}^{\prime} \cap \mathfrak{h}^{\prime}=\{0\} .
$$

Sejam $X \longmapsto X^{\prime}$ (resp. $Y \longmapsto Y^{\prime}$ ) o isomorfismo de $\mathfrak{g}$ sobre $\mathfrak{g}^{\prime}$ (resp. $\mathfrak{h}$ sobre $\mathfrak{h}^{\prime}$ ) correspondente ao isomorfismo $g \longmapsto g^{\prime}$ (resp. $h \longmapsto h^{\prime}$ ) de $G$ sobre $G^{\prime}$ (resp. $H$ sobre $H^{\prime}$ ). De (1.25) segue que

$$
\left(\exp \left(\tau_{h}(X)\right)\right)^{\prime}=h^{\prime} \exp \left(X^{\prime}\right)\left(h^{\prime}\right)^{-1},
$$

para quaisquer $h \in H$ e $X \in \mathfrak{g}$, do qual concluimos que

$$
\tau_{h}(X)^{\prime}=\operatorname{Ad}_{h^{\prime}}\left(X^{\prime}\right) .
$$

Diferenciando a relação (1.26), obtemos:

$$
(\sigma(Y) X)^{\prime}=\left[Y^{\prime}, X^{\prime}\right],
$$

para quaisquer $Y \in \mathfrak{h}$ e $X \in \mathfrak{g}$. A equação (1.27) implica que a aplicação

$$
(X, Y) \longmapsto X^{\prime}+Y^{\prime}
$$

é um isomorfismo de álgebras de Lie de $\mathfrak{h} \ltimes \mathfrak{g}$ sobre $\mathfrak{a}$.

Quando $G$ e $H$ são simplesmente conexos, vale a recíproca do Lema 1.3.2. Mais precisamente, temos o seguinte:

Lema 1.3.3 Sejam $G, H$ grupos de Lie simplesmente conexos com álgebras de Lie $\mathfrak{g}$ e $\mathfrak{h}$, respectivamente, e $\sigma: \mathfrak{h} \rightarrow \mathfrak{g l}(\mathfrak{g})$ uma representação de $\mathfrak{h}$ em $\mathfrak{g}$ tal que $\sigma(Y)$ é uma derivação de $\mathfrak{g}$, para todo $Y \in \mathfrak{h}$. Então, existe um único homomorfismo de grupos de Lie $\rho: H \rightarrow \operatorname{Aut}(G)$ tal que a álgebra de Lie $\mathfrak{h} \ltimes \mathfrak{g}$ é o produto semi-direto associado com $H \ltimes G$. 
Demonstração. Como $H$ é simplesmente conexo, existe uma representação $\tau: H \rightarrow \mathrm{GL}(\mathfrak{g})$ de $H$ em $\mathfrak{g}$ cuja diferencial é $\sigma$. Por outro lado, como cada $\tau_{h}$ é um automorfismo de $\mathfrak{g}$ e $G$ é simplesmente conexo, $\tau_{h}$ é a diferencial de um único automorfismo $\rho_{h}$ de $G$. Além disso, a aplicação $\rho: H \rightarrow \operatorname{Aut}(G)$, assim definida, é um homomorfismo de grupos de Lie. Podemos, assim, formar o produto semi-direto $H \ltimes G$ associado a $\rho$. Claramente $\mathfrak{h} \ltimes \mathfrak{g}$ é o produto semi-direto associado com $H \ltimes G$.

O produto semi-direto $H \ltimes G$, dado no Lema 1.3.3, é chamado o produto semi-direto associado com $\mathfrak{h} \ltimes \mathfrak{g}$. Finalizamos esta subseção com o seguinte:

Teorema 1.3.4 Seja $\mathfrak{g}$ uma álgebra de Lie sobre $\mathbb{R}$ ou $\mathbb{C}$. Então, existe um grupo de Lie simplesmente conexo $G$ cuja álgebra de Lie é isomorfa a $\mathfrak{g}$.

Demonstração. Ver [35, Teorema 3.15.1] ou [36, Teorema 3.28].

\subsubsection{Grupos de Lie nilpotentes}

Uma álgebra de Lie $\mathfrak{g}$ sobre $\mathbb{K}$ é chamada nilpotente se, para cada $X \in \mathfrak{g}$, $\operatorname{ad}_{X}: \mathfrak{g} \rightarrow \mathfrak{g}$ é um endomorfismo nilpotente. Toda álgebra de Lie abeliana é nilpotente. Mais geralmente, sejam $V$ um espaço vetorial de dimensão finita sobre $\mathbb{K}$ e $\mathfrak{g}$ uma subálgebra de $\mathfrak{g l}(V)$ consistindo de elementos nilpotentes. Então, $\mathfrak{g}$ é nilpotente e existe uma base $\left\{e_{1}, \ldots, e_{n}\right\}$ de $V$ em termos da qual todos os endomorfismos $X \in \mathfrak{g}$ são expressos por matrizes com zeros abaixo da diagonal principal.

Seja $\mathfrak{g}$ uma álgebra de Lie nilpotente de dimensão finita. Definimos, indutivamente, uma sequência de subconjuntos $\mathfrak{g}_{i}$ de $\mathfrak{g}$ como segue:

$$
\begin{aligned}
\mathfrak{g}_{0} & =\{0\}, \\
\mathfrak{g}_{i} & =\left\{X \in \mathfrak{g}:[X, \mathfrak{g}] \subseteq \mathfrak{g}_{i-1}\right\},
\end{aligned}
$$

para $i \geq 1$. Cada $\mathfrak{g}_{i}$ é um ideal em $\mathfrak{g}, \mathfrak{g}_{s}=\mathfrak{g}$ para algum inteiro $s$, com $1 \leq s \leq \operatorname{dim}(\mathfrak{g})$ e $\mathfrak{g}_{i} \subseteq \mathfrak{g}_{i+1}, 0 \leq i \leq s-1$ (ver [35, Teorema 3.5.4]).

Definimos também, indutivamente, uma sequência $\mathcal{C}^{k} \mathfrak{g}, k \geq 0$, como segue:

$$
\begin{aligned}
\mathcal{C}^{0} \mathfrak{g} & =\mathfrak{g}, \\
\mathcal{C}^{k} \mathfrak{g} & =\left[\mathfrak{g}, \mathcal{C}^{k-1} \mathfrak{g}\right],
\end{aligned}
$$

para $k \geq 1$. A série

$$
\mathcal{C}^{0} \mathfrak{g} \supset \mathcal{C}^{1} \mathfrak{g} \supset \mathcal{C}^{2} \mathfrak{g} \supset \ldots
$$

é chamada a série decrescente central de $\mathfrak{g}$. 
Proposição 1.3.5 Uma álgebra de Lie $\mathfrak{g}$ de dimensão finita é nilpotente se, e somente se, $\mathcal{C}^{k} \mathfrak{g}=\{0\}$ para algum $k \geq 1$.

Demonstração. Dados $X \in \mathfrak{g}$ e $k \geq 1$, temos que $\left(\operatorname{ad}_{X}\right)^{k}(\mathfrak{g}) \subset \mathcal{C}^{k} \mathfrak{g}$. Assim, se $\mathcal{C}^{k} \mathfrak{g}=\{0\}$, para algum $k \geq 1$, então $\operatorname{ad}_{X}$ é nilpotente para todo $X \in \mathfrak{g}$, mostrando que $\mathfrak{g}$ é nilpotente. Reciprocamente, suponha $\mathfrak{g}$ nilpotente. Seja $\mathfrak{g}_{i}, 1 \leq i \leq s$, os ideais definidos em (1.28). Claramente temos

$$
\mathcal{C}^{1} \mathfrak{g} \subseteq \mathfrak{g}_{s-1}, \quad \mathcal{C}^{2} \mathfrak{g} \subseteq \mathfrak{g}_{s-2}, \ldots,
$$

$\operatorname{assim} \mathcal{C}^{s} \mathfrak{g}=\{0\}$

Corolário 1.3.6 Toda álgebra de Lie nilpotente $\mathfrak{g} \neq\{0\}$ tem centro nãonulo.

Demonstração. Se $\mathcal{C}^{k} \mathfrak{g}=\{0\}$, para algum $k \geq 1$, então $\mathcal{C}^{k-1} \mathfrak{g}$ está contido no centro de $\mathfrak{g}$.

Definição 1.3.7 Um grupo de Lie $G$ é chamado nilpotente se sua álgebra de Lie $\mathfrak{g}$ é nilpotente. O menor inteiro $k$, dado na Proposição 1.3.5, é chamado o indice de nilpotência do grupo $G$.

Teorema 1.3.8 Seja $G$ um grupo de Lie nilpotente simplesmente conexo. Então, exp : $\mathfrak{g} \rightarrow G$ é um difeomorfismo diferenciável. Se $H$ é um subgrupo de Lie de $G$ e h é a correspondente subálgebra de $\mathfrak{g}$, então $H$ é fechado em $G$, é simplesmente conexo e $H=\exp (\mathfrak{h})$.

Demonstração. Ver [35, Teorema 3.6.2].

\subsubsection{Grupos de Lie solúveis}

Seja $\mathfrak{g}$ uma álgebra de Lie sobre $\mathbb{K}$. O espaço vetorial

$$
\mathcal{D} \mathfrak{g}=[\mathfrak{g}, \mathfrak{g}]=\operatorname{span}\{[X, Y]: X, Y \in \mathfrak{g}\}
$$

é uma subálgebra de $\mathfrak{g}$, chamada a álgebra derivada de $\mathfrak{g}$. Definimos $\mathcal{D}^{k} \mathfrak{g}$, $k \geq 0$, indutivamente, como segue:

$$
\begin{aligned}
\mathcal{D}^{0} \mathfrak{g} & =\mathfrak{g}, \\
\mathcal{D}^{k} \mathfrak{g} & =\mathcal{D}\left(\mathcal{D}^{k-1} \mathfrak{g}\right),
\end{aligned}
$$

para $k \geq 1$. Se $\mathfrak{h}$ é uma subálgebra de $\mathfrak{g}, \mathcal{D h}=[\mathfrak{h}, \mathfrak{h}]$ também é uma subálgebra de $\mathfrak{g}$, de modo que (1.30) define uma sequência

$$
\mathfrak{g} \supseteq \mathcal{D}^{1} \mathfrak{g} \supseteq \mathcal{D}^{2} \mathfrak{g} \supseteq \ldots
$$

de subálgebras de $\mathfrak{g} \cdot \mathcal{D}^{k} \mathfrak{g}$ é chamado a $k$-ésima álgebra derivada de $\mathfrak{g}$. 
Proposição 1.3.9 Se $\mathfrak{h}$ é um ideal de $\mathfrak{g}$ então $\mathcal{D}^{k} \mathfrak{h}$ são ideais de $\mathfrak{g}, k \geq 1$. Se $D$ é uma derivação de $\mathfrak{g}$ que deixa $\mathfrak{h}$ invariante, então $D$ deixa cada $\mathcal{D}^{k} \mathfrak{h}$ invariante. Em particular, os $\mathcal{D}^{k} \mathfrak{g}$ são ideais de $\mathfrak{g}$ invariantes sob qualquer derivação de $\mathfrak{g}$. Além disso, as álgebras $\mathcal{D}^{k} \mathfrak{g} / \mathcal{D}^{k+1} \mathfrak{g}$ são abelianas, $k \geq 0$.

Demonstração. Se $X, Y \in \mathfrak{h}$ e $Z \in \mathfrak{g}$, então $[Z,[X, Y]]=-[X,[Y, Z]]-$ $[Y,[Z, X]]$. Assim, $\mathcal{D h}$ é um ideal de $\mathfrak{g}$. Se $D$ é uma derivação de $\mathfrak{g}$ com $D(\mathfrak{h})=\mathfrak{h}$, então $D([X, Y])=[D(X), Y]+[X, D(Y)]$, mostrando que $D(\mathcal{D h})=$ $\mathcal{D h}$. Por indução sobre $k$, segue a afirmação. Finalmente, dados $X, Y \in \mathfrak{g}$, temos $[X, Y] \in \mathcal{D} \mathfrak{g}$, mostrando que $\mathfrak{g} / \mathcal{D} \mathfrak{g}$ é abeliana. Por indução sobre $k$ segue que $\mathcal{D}^{k} \mathfrak{g} / \mathcal{D}^{k+1} \mathfrak{g}$ é abeliana.

Definição 1.3.10 Uma álgebra de Lie $\mathfrak{g}$ é chamada solúvel se $\mathcal{D}^{k} \mathfrak{g}=\{0\}$, para algum inteiro $k \geq 0$. Um grupo de Lie $G$ é chamado solúvel se sua álgebra de Lie $\mathfrak{g}$ é solúvel. O menor inteiro $k$, tal que $\mathcal{D}^{k} \mathfrak{g}=\{0\}$, é chamado o indice de solubilidade de $G$.

Se $\mathfrak{g} \neq\{0\}$ é uma álgebra de Lie solúvel, com índice de solubilidade igual a $k$, então $\mathcal{D}^{k-1} \mathfrak{g}$ é um ideal abeliano não-nulo em $\mathfrak{g}$. Se $\mathfrak{a}$ é um subespaço de $\mathfrak{g}$, com $\mathcal{D} \mathfrak{g} \subseteq \mathfrak{a} \subseteq \mathfrak{g}$, então $[\mathfrak{g}, \mathfrak{a}] \subseteq \mathcal{D} \mathfrak{g} \subseteq \mathfrak{a}$, logo a é um ideal de $\mathfrak{g}$. Em particular, quando $\mathfrak{g}$ é solúvel, podemos escolher ideais $\mathfrak{a}$ em $\mathfrak{g}$ com $\operatorname{dim}(\mathfrak{g} / \mathfrak{a})=1$.

Os lemas seguintes nos dão caracterizações de álgebras de Lie solúveis.

Lema 1.3.11 Uma álgebra de Lie $\mathfrak{g}$ é solúvel se, e somente se, para cada ideal $\mathfrak{h} \neq\{0\}$ em $\mathfrak{g}$ existe um ideal $\mathfrak{h}_{1}$ de $\mathfrak{h}$ de codimensão 1 .

Demonstração. Ver [14, Lema 3.2.1].

Lema 1.3.12 Uma álgebra de Lie $\mathfrak{g}$ é solúvel se, e somente se, existem ideais $\mathfrak{g}_{0}=\mathfrak{g}, \mathfrak{g}_{1}, \ldots, \mathfrak{g}_{s+1}=\{0\}$, tais que $\mathfrak{g}_{k+1} \subseteq \mathfrak{g}_{k}$ e $\mathfrak{g}_{k} / \mathfrak{g}_{k+1}$ é abeliana, para $0 \leq k \leq s$.

Demonstração. Se $\mathfrak{g}$ é solúvel, basta definir $\mathfrak{g}_{k}=\mathcal{D}^{k} \mathfrak{g}, k \geq 0$, como em (1.30). Reciprocamente, sejam $\mathfrak{g}_{0}, \mathfrak{g}_{1}, \ldots, \mathfrak{g}_{s+1}$ como no enunciado. Como $\mathfrak{g}_{k+1} / \mathfrak{g}_{k}$ é abeliano tem-se $\mathcal{D} \mathfrak{g}_{k} \subseteq \mathfrak{g}_{k+1}$. Assim, $\mathcal{D}^{k} \mathfrak{g} \subseteq \mathfrak{g}_{k}, k \geq 0$, mostrando que $\mathcal{D}^{s+1} \mathfrak{g}=\{0\}$, ou seja, $\mathfrak{g}$ é solúvel.

Corolário 1.3.13 Todo grupo de Lie nilpotente $G$ é solúvel. 
Demonstração. Seja $\mathfrak{g}$ a álgebra de Lie de $G$. Consideremos os ideais $\mathfrak{g}_{k}$ de $\mathfrak{g}$ definidos em (1.28). Como $\mathcal{D} \mathfrak{g}_{k} \subseteq\left[\mathfrak{g}, \mathfrak{g}_{k}\right] \subseteq \mathfrak{g}_{k+1}, \mathfrak{g}_{k+1} / \mathfrak{g}_{k}$ é abeliana para $k \geq 0$. Como $\mathfrak{g}_{0}=\{0\}$ e $\mathfrak{g}_{s}=\mathfrak{g}$, para algum $s \geq 1$ suficientemente grande, $\mathfrak{g}$ é solúvel pelo Lema 1.3.12.

Lema 1.3.14 Uma álgebra de Lie $\mathfrak{g}$ é solúvel se, e somente se,

$$
\langle X,[Y, Z]\rangle=0,
$$

para quaisquer $X, Y, Z \in \mathfrak{g}$, onde $\langle\cdot, \cdot\rangle$ denota a forma de Killing de $\mathfrak{g}$ (cf. Definição 1.2.9). Em particular, se a forma de Killing de $\mathfrak{g}$ é identicamente nula, então $\mathfrak{g}$ é solúvel.

Demonstração. Ver [35, Teorema 3.9.1].

\subsection{Grupos de Lie clássicos}

Nesta seção apresentaremos os grupos de Lie clássicos e suas álgebras de Lie que serão utilizados no texto. Tais grupos e álgebras serão constituídos por matrizes reais (ou por operadores lineares sobre $\mathbb{R}$ ). Os espaços vetoriais considerados abaixo serão sempre de dimensão finita.

Exemplo 1.4.1 A reta real $\mathbb{R}$ é um grupo de Lie com a operação de soma de números reais. Os campos vetoriais invariantes à esquerda são simplesmente os campos vetoriais constantes $\lambda\left(\frac{\mathrm{d}}{\mathrm{d} t}\right), \lambda \in \mathbb{R}$. O colchete de quaisquer dois de tais campos vetoriais é nulo.

Exemplo 1.4.2 Dado um espaço vetorial real $V$, denotemos por GL $(V)$ o grupo de todos os isomorfismos lineares (sobre $\mathbb{R}$ ) de $V$. Sua álgebra de Lie, denotada por $\mathfrak{g l}(V)$, coincide com o espaço vetorial $\operatorname{Lin}(V)$ dos endomorfismos lineares de $V$. Dizemos que $\mathrm{GL}(V)$ é o grupo linear geral de $V$. Podemos identificar GL $\left(\mathbb{R}^{n}\right)$ com o grupo das matrizes reais invertíveis $n \times n$ e $\mathfrak{g l}\left(\mathbb{R}^{n}\right)$ com a álgebra de Lie das matrizes reais $n \times n$.

Exemplo 1.4.3 Dado um espaço vetorial real $V$, denotemos por $\mathrm{GL}_{+}(V)$ o subgrupo de $\mathrm{GL}(V)$ formado pelos isomorfismos lineares de $V$ que preservam orientação, i.e., pelos isomorfismos lineares de determinante positivo. Segue que $\mathrm{GL}_{+}(V)$ é aberto em $\mathrm{GL}(V)$ e, assim, sua álgebra de Lie coincide com a álgebra de Lie $\mathfrak{g l}(V)$ de $\mathrm{GL}(V)$. Identificamos $\mathrm{GL}_{+}\left(\mathbb{R}^{n}\right)$ com o grupo das matrizes reais com determinante positivo. O grupo $\operatorname{GL}\left(\mathbb{R}^{n}\right)$ tem duas componentes conexas: $\mathrm{GL}_{+}\left(\mathbb{R}^{n}\right)$ e seu complementar. 
Exemplo 1.4.4 Sejam $V$ um espaço vetorial real, $W \subset V$ um subespaço vetorial e consideremos $\mathrm{GL}(V ; W)$ o sugbrupo de $\mathrm{GL}(V)$ formado pelos isomorfismos lineares $T: V \rightarrow V$ tais que $T(W)=W$. A álgebra de Lie $\mathfrak{g l}(V ; W)$ de $\mathrm{GL}(V ; W)$ é a subálgebra de Lie de $\mathfrak{g l}(V)$ formada por todos os endomorfismos lineares $T: V \rightarrow V$ tais que $T(W) \subset W$.

Exemplo 1.4.5 Dado um espaço vetorial real $V$, denotemos por $\operatorname{SL}(V)$ o subgrupo fechado de $\mathrm{GL}(V)$ formado pelos isomorfismos lineares de $V$ com determinante igual a 1. O grupo $\mathrm{SL}(V)$ é chamado o grupo linear especial de $V$. Sua álgebra de Lie $\mathfrak{s l}(V) \subset \mathfrak{g l}(V)$ é formada pelos endomorfismos lineares de $V$ de traço zero. Denotaremos por $\mathrm{SL}\left(\mathbb{R}^{n}\right)=\mathrm{SL}_{n}(\mathbb{R})$ e $\mathfrak{s l}\left(\mathbb{R}^{n}\right)=\mathfrak{s} l_{n}(\mathbb{R})$. Assim, podemos identificar $\mathrm{SL}_{n}(\mathbb{R})$ com o grupo das matrizes reais $n \times n$ com determinante igual a 1 , e $\mathfrak{s} l_{n}(\mathbb{R})$ com a álgebra de Lie das matrizes reais $n \times n$ de traço nulo. Além disso, $\mathrm{SL}_{n}(\mathbb{R})$ é um grupo de Lie conexo.

Exemplo 1.4.6 Seja $V$ um espaço vetorial real munido de um produto escalar $g$ de índice $r$. Denotemos por $\mathrm{O}_{r}(V, g)$ (ou, simplesmente, por $\mathrm{O}_{r}(V)$, subentendendo o produto escalar $g$ ) o subgrupo fechado de $\mathrm{GL}(V)$ formado pelos isomorfismos lineares de $V$ que são ortogonais em relação a $g \cdot \mathrm{O}_{r}(V)$ é chamado o grupo ortogonal de $V$ relativo a $g$. A álgebra de Lie de $\mathrm{O}_{r}(V)$, denotada por $\mathfrak{o}_{r}(V)$, é a subálgebra de $\mathfrak{g l}(V)$ formada pelos endomorfismos lineares de $V$ que são anti-simétricos em relação a $g$. Se $V=\mathbb{R}^{n}$ e $g=\langle\cdot, \cdot\rangle$ é a forma bilinear de Minkowski de índice $r$, i.e.,

$$
\langle x, y\rangle=\sum_{i=1}^{n-r} x_{i} y_{i}-\sum_{i=n-r+1}^{n} x_{i} y_{i},
$$

para quaisquer $x, y \in \mathbb{R}^{n}$, então $\mathrm{O}_{r}\left(\mathbb{R}^{n}\right)$ é o grupo das matrizes reais $n \times$ $n$ que são ortogonais em relação a $\langle\cdot, \cdot\rangle$, e $\mathfrak{o}_{r}\left(\mathbb{R}^{n}\right)$ é a álgebra de Lie das matrizes reais $n \times n$ que são anti-simétricas em relação a $\langle\cdot, \cdot\rangle$. Além disso, o grupo $\mathrm{O}_{r}\left(\mathbb{R}^{n}\right)$ é compacto pois é fechado e limitado no espaço Euclidiano das matrizes reais $n \times n$. Quando $r=0$, i.e., $g$ é um produto escalar definido positivo, denotaremos por $\mathrm{O}_{r}(V)=\mathrm{O}(V)$ e $\mathfrak{o}_{r}(V)=\mathfrak{o}(V)$.

Exemplo 1.4.7 Seja $V$ um espaço vetorial real munido de um produto escalar $g$ de índice $r$. Denotemos por $\mathrm{SO}_{r}(V)$ o subgrupo de $\mathrm{GL}(V)$ definido por

$$
\mathrm{SO}_{r}(V)=\mathrm{O}_{r}(V) \cap \mathrm{SL}(V)=\mathrm{O}_{r}(V) \cap \mathrm{GL}_{+}(V) .
$$

$\mathrm{SO}_{r}(V)$ é chamado o grupo ortogonal especial. Sua álgebra de Lie, denotada por $\mathfrak{s o}_{r}(V)$, coincide com $\mathfrak{o}_{r}(V)$, pois $\mathrm{SO}_{r}(V)$ é aberto em $\mathrm{O}_{r}(V)$. O grupo 
$\mathrm{SO}_{r}\left(\mathbb{R}^{n}\right)$ é conexo e $\mathrm{O}_{r}\left(\mathbb{R}^{n}\right)$ tem duas componentes conexas: $\mathrm{SO}_{r}\left(\mathbb{R}^{n}\right)$ e seu complementar.

Exemplo 1.4.8 O grupo de Heisenberg de dimensão 3, denotado por $\mathrm{Nil}_{3}$ (ou, simplesmente, por Nil), é o subgrupo do grupo das matrizes reais $3 \times 3$ definido por

$$
\mathrm{Nil}=\left\{\left(\begin{array}{ccc}
1 & x & z \\
0 & 1 & y \\
0 & 0 & 1
\end{array}\right): x, y, z \in \mathbb{R}\right\},
$$

com a multiplicação usual de matrizes. Assim, identificando a matriz (1.31) com a terna $(x, y, z) \in \mathbb{R}^{3}$, temos:

$$
(x, y, z) \cdot\left(x^{\prime}, y^{\prime}, z^{\prime}\right)=\left(x+x^{\prime}, y+y^{\prime}, z+z^{\prime}+x y^{\prime}\right) .
$$

O elemento identidade de Nil é $0=(0,0,0)$ e o elemento inverso de $(x, y, z)$ é $(x, y, z)^{-1}=(-x,-y, x y-z)$. Dados $a, b \in \mathrm{Nil}$, com $a=(x, y, z) \mathrm{e}$ $b=\left(x^{\prime}, y^{\prime}, z^{\prime}\right)$, o comutador $[a, b]$ dos elementos $a$ e $b$ é igual a

$$
[a, b]=a b a^{-1} b^{-1}=\left(0,0, x y^{\prime}-y x^{\prime}\right),
$$

onde $x y^{\prime}-y x^{\prime} \neq 0$, em geral. Por exemplo, se $a=(1,0,0)$ e $b=(0,1,0)$, temos $[a, b]=(0,0,1) \neq 0$. Isso mostra que Nil não é abeliano. Por outro lado, dados $a, b, c \in \mathrm{Nil}$, o duplo comutador de $a, b, c$ é igual a

$$
[[a, b], c]=(0,0,0),
$$

ou seja, Nil é um grupo de Lie nilpotente com índice de nilpotência igual a 2 . Cada ponto $(x, y, z) \in$ Nil pode ser visto como uma translação (à esquerda) da identidade a esse ponto como sendo:

$$
(x, y, z) \cdot(0,0,0)=(x, y, z)
$$

ou seja, $L_{(x, y, z)}(0)=(x, y, z)$. Então, as direções coordenadas Euclidianas são transladadas para:

$$
\begin{aligned}
& (x, y, z) \cdot(s, 0,0)=(x+s, y, z) \\
& (x, y, z) \cdot(0, s, 0)=(x, y+s, z+x s) \\
& (x, y, z) \cdot(0,0, s)=(x, y, z+s)
\end{aligned}
$$


Diferenciando (em relação a $s$ ), obtemos os campos vetoriais:

$$
\begin{aligned}
& E_{1}=\frac{\partial}{\partial x}, \\
& E_{2}=\frac{\partial}{\partial y}+x \frac{\partial}{\partial z}, \\
& E_{3}=\frac{\partial}{\partial z},
\end{aligned}
$$

que são campos vetoriais invariantes à esquerda, por construção. Portanto, a álgebra de Lie de Nil, denotada por nil, é gerada pelos campos vetoriais $E_{1}, E_{2}, E_{3}$, dados em (1.32), cujos colchetes de Lie são dados por:

$$
\left[E_{1}, E_{2}\right]=E_{3} \quad \text { e } \quad\left[E_{3}, E_{2}\right]=\left[E_{3}, E_{1}\right]=0 .
$$

Exemplo 1.4.9 O grupo de $\mathrm{Lie} \mathrm{Sol}_{3}$ (ou, simplesmente, Sol) é o produto semi-direto $\mathbb{R} \ltimes \mathbb{R}^{2}$, onde $z \in \mathbb{R}$ age em $\mathbb{R}^{2}$ através da aplicação $\rho_{z}$ definida por

$$
\rho_{z}(x, y)=\left(e^{z} x, e^{-z} y\right)
$$

para quaisquer $x, y \in \mathbb{R}$. Para cada $z \in \mathbb{R}, \rho_{z}$ é um isomorfismo linear de $\mathbb{R}^{2}$. Identificando Sol com $\mathbb{R}^{3}$, de modo que o plano- $x y$ corresponda ao subgrupo normal $\mathbb{R}^{2}$, a multiplicação do grupo Sol, induzida por (1.33), é dada por

$$
(x, y, z) \cdot\left(x^{\prime}, y^{\prime}, z^{\prime}\right)=\left(x+e^{z} x^{\prime}, y+e^{-z} y^{\prime}, z+z^{\prime}\right),
$$

para quaisquer $(x, y, z),\left(x^{\prime}, y^{\prime}, z^{\prime}\right) \in \mathbb{R}^{3}$ (cf. (1.13)). Claramente, $(0,0,0)$ é o elemento identidade de Sol, e o elemento inverso é

$$
(x, y, z)^{-1}=\left(-e^{-z} x,-e^{z} y,-z\right) .
$$

A ação à esquerda do grupo Sol nas direções coordendas Euclidianas produz:

$$
\begin{aligned}
& (x, y, z) \cdot(s, 0,0)=\left(x+e^{z} s, y, z\right), \\
& (x, y, z) \cdot(0, s, 0)=\left(x, y+e^{-z} s, z\right), \\
& (x, y, z) \cdot(0,0, s)=(x, y, z+s) .
\end{aligned}
$$

Diferenciando em relação a $s$, obtemos os campos vetoriais:

$$
\begin{aligned}
& E_{1}=e^{z} \frac{\partial}{\partial x}, \\
& E_{2}=e^{-z} \frac{\partial}{\partial y}, \\
& E_{3}=\frac{\partial}{\partial z}
\end{aligned}
$$


que são campos invariantes à esquerda, por construção. Portanto, a álgebra de Lie do grupo de Lie Sol, denotada por $\mathfrak{s o l}$, é gerada pelos campos vetoriais $E_{1}, E_{2}, E_{3}$ dados em (1.35), cujos colchetes de Lie são dados por

$$
\left[E_{3}, E_{1}\right]=E_{1}, \quad\left[E_{3}, E_{2}\right]=-E_{2}, \quad\left[E_{1}, E_{2}\right]=0 .
$$

O grupo Sol é um grupo de Lie solúvel. De fato, de (1.36), a álgebra derivada $\mathcal{D} \mathfrak{s o l}$ é dada por

$$
\mathcal{D} \mathfrak{s o l}=[\mathfrak{s o l}, \mathfrak{s o l}]=\operatorname{span}\left\{E_{1}, E_{2}\right\} .
$$

Novamente, usando (1.36), a álgebra derivada $\mathcal{D}^{2} \mathfrak{s o l}$ é igual a

$$
\mathcal{D}^{2} \mathfrak{s o l}=\operatorname{span}\left\{\left[E_{1}, E_{2}\right]\right\}=\{0\} .
$$

Portanto, Sol é um grupo de Lie solúvel, com índice de solubilidade igual a 2.

\subsection{Variedades homogêneas}

Sejam $G$ um grupo e $M$ um conjunto arbitrário. Uma ação à esquerda de $G$ em $M$ é uma aplicação

$$
(g, x) \in G \times M \longmapsto g \cdot x \in M
$$

tal que $g_{1}\left(g_{2} \cdot x\right)=\left(g_{1} g_{2}\right) \cdot x$ e $1 \cdot x=x$, para quaisquer $g_{1}, g_{2} \in G$ e $x \in M$.

Dado uma ação à esquerda de $G$ em $M$, obtemos, para cada elemento $x \in M$, uma aplicação

$$
\beta_{x}: G \rightarrow M
$$

definida pela ação no elemento $x \in M$, i.e., $\beta_{x}(g)=g \cdot x$, para todo $g \in G$ e, para cada $g \in G$, obtemos uma bijeção

$$
\gamma_{g}: M \rightarrow M
$$

correspondente à ação do elemento $g \in G$, i.e., $\gamma_{g}(x)=g \cdot x$, para todo $x \in M$.

Se $\operatorname{Bij}(M)$ denota o grupo das bijeções de $M$, munido com a operação de composição, então a aplicação

$$
g \in G \longmapsto \gamma_{g} \in \operatorname{Bij}(M)
$$

é um homomorfismo de grupos. 
Para cada $x \in M$, definimos a $G$-órbita (ou, simplesmente, a órbita) de $x$ relativo a ação de $G$ por:

$$
G(x)=\{g \cdot x: g \in G\}=\beta_{x}(G) .
$$

As órbitas da ação de $G$ em $M$ constituem uma partição do conjunto $M$. Definimos também o subgrupo de isotropia do elemento $x \in M$ por:

$$
G_{x}=\{g \in G: g \cdot x=x\}=\beta_{x}^{-1}(x) ;
$$

claramente $G_{x}$ é um subgrupo de $G$.

Dizemos que ação de $G$ em $M$ é transitiva quando $G(x)=M$ para algum (e, portanto, para todo) $x \in M$. Dizemos que a ação de $G$ em $M$ é livre quando $G_{x}=\{1\}$, para todo $x \in M$. Dizemos também que a ação de $G$ em $M$ é efetiva quando o homomorfismo (1.40) é bijetor, i.e., quando

$$
\bigcap_{x \in M} G_{x}=\{1\}
$$

Se $H$ é um subgrupo de $G$, denotemos por $G / H$ o conjunto das classes laterais à esquerda de $H$ em $G$ :

$$
G / H=\{g H: g \in G\},
$$

onde

$$
g H=\{g h: h \in H\}
$$

é a classe lateral à esquerda do elemento $g \in G$. Temos uma ação natural de $G$ em $G / H$ dada por:

$$
\left(g_{1}, g_{2}\right) \in G \times G / H \longmapsto\left(g_{1} g_{2}\right) H \in G / H
$$

a ação (1.43) é chamada a ação por translação à esquerda de $G$ nas classes laterais à esquerda de $H$. A ação (1.43) é sempre transitiva.

Se $G$ age à esquerda em $M$ então, para cada $x \in M$, a aplicação $\beta_{x}$, definida em (1.38), passa ao quociente e define uma bijeção

$$
\bar{\beta}_{x}: G / G_{x} \rightarrow G(x)
$$

dada por $\bar{\beta}_{x}\left(g G_{x}\right)=g \cdot x$, onde $G_{x}$ denota o subgrupo de isotropia do elemento $x \in M$. Temos, assim, o seguinte diagrama comutativo:

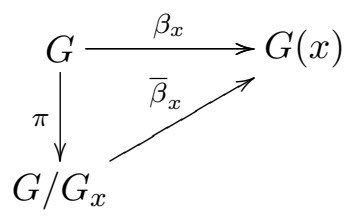

onde $\pi: G \rightarrow G / G_{x}$ denota a aplicação quociente. 
Observação 1.5.1 De forma similar, define-se uma ação à direita de $G$ em $M$ como sendo uma aplicação

$$
(x, g) \in M \times G \longmapsto x \cdot g \in M
$$

satisfazendo $\left(x \cdot g_{1}\right) \cdot g_{2}=x \cdot\left(g_{1} g_{2}\right)$ e $x \cdot 1=x$, para quaisquer $g_{1}, g_{2} \in G$ e $x \in M$. Uma teoria totalmente análoga à de ações à esquerda pode ser desenvolvida para ações à direita; de fato, à uma ação à direita (1.45) pode-se sempre associar uma ação à esquerda definida por

$$
(g, x) \in G \times M \longmapsto x \cdot g^{-1} \in M .
$$

Note que na teoria de ações à direita, para que a bijeção (1.44) esteja bem definida deve-se entender o quociente $\mathrm{G} / \mathrm{H}$ como o conjunto de classes laterais à direita $H g, g \in G$, onde

$$
H g=\{h g: g \in G\}
$$

Sejam agora $G$ um grupo de Lie, $M$ uma variedade diferenciável e suponha que $G$ age à esquerda em $M$; neste contexto suporemos que aplicação (1.37) é diferenciável. Se $H$ é um subgrupo fechado de $G$ então existe uma única estrutura de variedade diferenciável no conjunto $G / H$ tal que a aplicação quociente

$$
\pi: G \rightarrow G / H
$$

é uma submersão diferenciável (ver [36, Teorema 3.58]). O núcleo da diferencial $\mathrm{d} \pi_{1}$ é precisamente a álgebra de Lie $\mathfrak{h}$ de $H$, de modo que o espaço tangente a $G / H$ no elemento identidade $1 H$ pode ser identificado com o espaço quociente $\mathfrak{g} / \mathfrak{h}$. Observe que, como $\pi$ é sobrejetora e aberta (ver [3, Teorema 3.7.12]), segue que $G / H$ possui a topologia quociente da topologia de $G$.

Se um grupo de Lie $G$ age à esquerda numa variedade diferenciável $M$ então, para cada $x \in M$, o subgrupo de isotropia $G_{x}$ é um subgrupo fechado de $G$, donde temos uma estrutura de variedade diferenciável em $G / G_{x}$. Assim, para cada $x \in M$, a órbita $G(x)$ possui uma única estrutura de variedade diferenciável que torna a bijeção $\bar{\beta}_{x}$, definida em (1.44), um difeomorfismo diferenciável ${ }^{1}$. Com essa estrutura, $G(x)$ torna-se uma subvariedade imersa de $M$ e o espaço tangente $T_{x} G(x)$ coincide com a imagem da aplicação

$$
\mathrm{d} \beta_{x}(1): \mathfrak{g} \rightarrow T_{x} M
$$

\footnotetext{
${ }^{1}$ Escolhendo-se outro ponto $y \in G(x)$, com $G(x)=G(y)$, então a estrutura de variedade diferenciável induzida em $G(x)$ por $\bar{\beta}_{y}$ coincide com a estrutura induzida por $\bar{\beta}_{x}$.
} 
(ver [36, Teorema 3.62]). Em particular, se $G$ age transitivamente em $M$ então, para cada $x \in M$, a aplicação (1.44) é um difeomorfismo diferenciável de $G / G_{x}$ sobre $M$. Além disso, a aplicação $\beta_{x}$, dada em (1.38), é uma submersão sobrejetora.

Definição 1.5.2 Variedades diferenciáveis da forma $G / H$, onde $G$ é um grupo de Lie, $H$ é um subgrupo fechado de $G$ e a estrutura diferenciável em $G / H$ é a única que torna a projeção $\pi: G \rightarrow G / H$ uma submersão diferenciável, são chamadas variedades homogêneas.

Se $G$ é um grupo de Lie e $H$ um subgrupo normal fechado de $G$ então a variedade homogênea $G / H$, com sua estrutura natural de grupo, é um grupo de Lie (ver [36, Teorema 3.64]).

O Lema seguinte estabelece a conexão entre subgrupos normais de $G$ e ideais em sua álgebra de Lie $\mathfrak{g}$.

Lema 1.5.3 Sejam $G$ um grupo de Lie com álgebra de Lie $\mathfrak{g}, H$ um subgrupo de Lie de $G$ e h a correspondente subálgebra de $\mathfrak{g}$. Então, $H$ é um subgrupo normal de $G$ se, e somente se, $\mathfrak{h}$ é um ideal de $\mathfrak{g}$.

Demonstração. Ver [35, Teorema 2.13.4].

\subsection{Métricas semi-Riemannianas $G$-invariantes}

Consideremos uma ação à esquerda de um grupo de Lie $G$ em uma variedade diferenciável $M$.

Definição 1.6.1 Dizemos que uma métrica semi-Riemanniana em $M$ é $G$ invariante se, para todo $g \in G$, a aplicação $\gamma_{g}: M \rightarrow M$, definida em (1.39), é uma isometria. Dizemos também que a ação de $G$ preserva a métrica semi-Riemanniana.

Seja $M$ uma variedade semi-Riemanniana e consideremos a ação transitiva de um grupo de Lie $G$ em $M$ que preserva a métrica semi-Riemanniana. Dado um ponto $x \in M$, seja $H$ o subgrupo de isotropia de $x$. Então, o difeomorfismo $\bar{\beta}_{x}: G / H \rightarrow M$, definido em (1.44), define uma única métrica semi-Riemanniana em $G / H$ que o torna uma isometria. A ação de $G$ em $M$ corresponde então à ação de $G$ em $G / H$ por translação à esquerda:

$$
g \cdot h H=(g h) H .
$$


Além disso, a métrica semi-Riemanniana em $G / H$ também é $G$-invariante (em relação à ação de $G$ por translação à esquerda).

Seguindo a Definição 1.5.2, não distinguiremos $M$ de $G / H$ e consideraremos apenas variedades semi-Riemannianas $G / H$, onde $G$ é um grupo de Lie, $H$ é um subgrupo fechado de $G$ e a métrica semi-Riemanniana é $G$ invariante, estando subentendido que a ação considerada de $G \mathrm{em} G / H$ é por translação à esquerda.

Dado $g \in G$, denotemos por

$$
\bar{L}_{g}: G / H \rightarrow G / H
$$

a translação à esquerda dada pela ação de $g$ em $G / H$, i.e., $\bar{L}_{g}(h H)=(g h) H$. Denotemos também por

$$
\operatorname{Ad}_{g}: \mathfrak{g} \rightarrow \mathfrak{g}
$$

a diferencial do automorfismo interno $\mathcal{I}_{g}: G \rightarrow G$, definido em (1.18), no elemento identidade $1 \in G ; \operatorname{Ad}_{g}$ é um isomorfismo de álgebras de Lie para todo $g \in G$. A aplicação

$$
\mathrm{Ad}: G \rightarrow \mathrm{GL}(\mathfrak{g}),
$$

definida por $\operatorname{Ad}(g)=\operatorname{Ad}_{g}$, para todo $g \in G$, é uma representação de $G$ em $\mathfrak{g}$, chamada a representação adjunta de $G$ em $\mathfrak{g}$. Sua diferencial no elemento identidade $1 \in G$ coincide com a representação adjunta de $\mathfrak{g}$ em $\mathfrak{g}$, i.e., $\mathrm{d}(\operatorname{Ad})(1)=$ ad. Mais precisamente, dados $X, Y \in \mathfrak{g}$, temos:

$$
[X, Y]=\lim _{t \rightarrow 0} \frac{1}{t}\left(\operatorname{Ad}_{\rho(t)} Y-Y\right),
$$

onde $\rho(t)=\exp (t X), t \in \mathbb{R}$, é o subgrupo a 1-parâmetro de $X$ (ver [36, Proposição 3.47]).

Definição 1.6.2 Um objeto definido na álgebra de Lie $\mathfrak{g}$ de $G$ é chamado $\operatorname{Ad}(H)$-invariante se ele é preservado por $\operatorname{Ad}_{h}: \mathfrak{g} \rightarrow \mathfrak{g}$, para todo $h \in H$.

Seja $\mathfrak{h} \subset \mathfrak{g}$ a álgebra de Lie de $H$.

Lema 1.6.3 Se uma forma bilinear simétrica $\langle\cdot, \cdot\rangle$ em $\mathfrak{g}$ é $\operatorname{Ad}(H)$-invariante, então

$$
\langle[X, Y], Z\rangle=-\langle Y,[X, Z]\rangle
$$

para todo $X \in \mathfrak{h}$ e para quaisquer $Y, Z \in \mathfrak{g}$. A recíproca vale se $H$ é conexo. 
Demonstração. Pela fórmula de Polarização, a equação (1.50) é equivalente a

$$
\langle[Y, X], X\rangle=0
$$

para quaisquer $Y \in \mathfrak{h}$ e $X \in \mathfrak{g}$. Supondo $\langle\cdot, \cdot\rangle \operatorname{Ad}(H)$-invariante, a função

$$
f(t)=\left\langle\operatorname{Ad}_{\rho(t)} X, \operatorname{Ad}_{\rho(t)} X\right\rangle
$$

é constante, igual a $\langle X, X\rangle$, onde $\rho(t)=\exp (t Y)$ é o subgrupo a 1-parâmetro de $Y$. Por outro lado, usando (1.49), temos:

$$
\langle[Y, X], X\rangle=f^{\prime}(t)=0,
$$

verificando (1.51) e, consequentemente, mostrando a igualdade (1.50). Reciprocamente, suponhamos válido (1.50). Como exp é um difeomorfismo local de uma vizinhança de $0 \in \mathfrak{g}$ sobre uma vizinhança de $1 \in G$, segue que $\langle\cdot, \cdot\rangle$ é $\operatorname{Ad}_{h}$-invariante para todo $h$ em alguma vizinhança $U$ da identidade $1_{H} \in H$. Agora, como $H$ é conexo e $h \in H \longmapsto \operatorname{Ad}_{h} \in \mathrm{GL}(\mathfrak{g})$ é um homeomorfismo, o resultado segue do fato ${ }^{2}$ que todo elemento de $H$ pode ser expresso como um produto finito de elementos de $U$.

Observação 1.6.4 A equação (1.50) afirma que $\operatorname{Ad}_{X}$ é anti-simétrico em relação a $\langle\cdot, \cdot\rangle$, para todo $X \in \mathfrak{h}$.

Exemplo 1.6.5 A forma de Killing $\langle\cdot, \cdot\rangle$ de $\mathfrak{g}$, definida no Exemplo 1.2.9, é $\operatorname{Ad}(G)$-invariante. De fato, a identidade de Jacobi (1.4) mostra que

$$
\operatorname{ad}_{[X, Y]}=\left[\operatorname{ad}_{X}, \operatorname{ad}_{Y}\right]
$$

para quaisquer $X, Y \in \mathfrak{g}$. Assim, dados $X, Y, Z \in \mathfrak{g}$, temos:

$$
\begin{aligned}
\langle[X, Y], Z\rangle & =\operatorname{tr}\left(\operatorname{ad}_{[X, Y]} \operatorname{ad}_{Z}\right)=\operatorname{tr}\left(\left[\operatorname{ad}_{X}, \operatorname{ad}_{Y}\right] \operatorname{ad}_{Z}\right) \\
& =\operatorname{tr}\left(\operatorname{ad}_{X} \operatorname{ad}_{Y} \operatorname{ad}_{Z}\right)-\operatorname{tr}\left(\operatorname{ad}_{Y} \operatorname{ad}_{X} \operatorname{ad}_{Z}\right) \\
& =-\operatorname{tr}\left(\operatorname{ad}_{Y} \operatorname{ad}_{X} \operatorname{ad}_{Z}\right)+\operatorname{tr}\left(\operatorname{ad}_{Y} \operatorname{ad}_{Z} \operatorname{ad}_{X}\right) \\
& =-\operatorname{tr}\left(\operatorname{ad}_{Y}\left(\operatorname{ad}_{X} \operatorname{ad}_{Z}-\operatorname{ad}_{Z} \operatorname{ad}_{X}\right)\right)=-\operatorname{tr}\left(\operatorname{ad}_{Y}\left[\operatorname{ad}_{X}, \operatorname{ad}_{Z}\right]\right) \\
& =-\operatorname{tr}\left(\operatorname{ad}_{Y} \operatorname{ad}_{[X, Z]}\right)=-\langle Y,[X, Z]\rangle .
\end{aligned}
$$

\footnotetext{
${ }^{2}$ Aqui, estamos usando o caso particular de um fato mais geral: Sejam $G$ um grupo de Lie e $G_{0}$ a componente conexa de $G$ que contém o elemento identidade $1 \in G$. Dado qualquer vizinhança $U$ de $1 \in G$, todo elemento de $G_{0}$ pode ser expresso como um produto finito de elementos de $U$ (ver [36, Proposição 3.18]).
} 
Seja $G / H$ um espaço homogêneo. Fixemos um subespaço $\mathfrak{m}$ de $\mathfrak{g}$ com $\mathfrak{g}=\mathfrak{h} \oplus \mathfrak{m}$ e denotemos por $\operatorname{pr}_{\mathfrak{h}}: \mathfrak{g} \rightarrow \mathfrak{h}$ e $\mathrm{pr}_{\mathfrak{m}}: \mathfrak{g} \rightarrow \mathfrak{m}$ as projeções correspondentes. A restrição da diferencial $\mathrm{d} \pi(1)$ a $\mathfrak{m}$ é um isomorfismo linear entre $\mathfrak{m}$ e $T_{1 H}(G / H)$. Assim, identificaremos $T_{1 H}(G / H)$ com $\mathfrak{m}$ através deste isomorfismo.

Dado $h \in H$, temos $\mathcal{I}_{h}(H) \subset H$, donde $\operatorname{Ad}_{h}(\mathfrak{h}) \subset \mathfrak{h}$ e, portanto, temos um isomorfismo induzido

$$
\overline{\operatorname{Ad}}_{h}: \mathfrak{m} \rightarrow \mathfrak{m},
$$

definido por:

$$
\overline{\operatorname{Ad}}_{h}=\left.\operatorname{pr}_{\mathfrak{m}} \circ \operatorname{Ad}_{h}\right|_{\mathfrak{m}} .
$$

A aplicação

$$
\overline{\mathrm{Ad}}: H \rightarrow \mathrm{GL}(\mathfrak{m}),
$$

definida por $\overline{\operatorname{Ad}}(h)=\overline{\operatorname{Ad}}_{h}$, para todo $h \in H$, é uma representação de $H$ em $\mathfrak{m}$, chamada a representação isotrópica de $H \mathrm{em} \mathfrak{m}$. A diferencial de $\overline{\mathrm{Ad}}$ no elemento identidade será denotada por

$$
\overline{\mathrm{ad}}: \mathfrak{h} \rightarrow \mathfrak{g l}(\mathfrak{m}) .
$$

Temos:

$$
\overline{\operatorname{ad}}_{X}(Y)=\operatorname{pr}_{\mathfrak{m}}([X, Y]),
$$

para quaisquer $X \in \mathfrak{h}$ e $Y \in \mathfrak{m}$. Além disso, diferenciando o seguinte diagrama comutativo

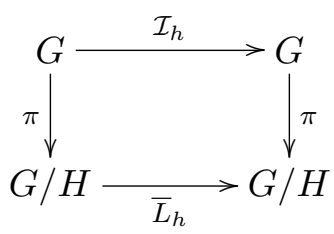

no elemento identidade, obtemos:

$$
\mathrm{d} \bar{L}_{h}(1 H)=\overline{\operatorname{Ad}}_{h},
$$

para todo $h \in H$.

Com a notação fixada acima, temos um Corolário imediato do Lema 1.6.3: 
Corolário 1.6.6 Sejam $G / H$ um espaço homogêneo e $\mathfrak{m}$ um subespaço de $\mathfrak{g}$, com $\mathfrak{g}=\mathfrak{h} \oplus \mathfrak{m}$. Se uma forma bilinear simétrica $\langle\cdot, \cdot\rangle$ em $\mathfrak{m}$ é $\overline{\operatorname{Ad}}(H)$ invariante então

$$
\left\langle\operatorname{pr}_{\mathfrak{m}}([X, Y]), Z\right\rangle=-\left\langle Y, \operatorname{pr}_{\mathfrak{m}}([X, Z])\right\rangle,
$$

para quaisquer $X \in \mathfrak{h}$ e $Y, Z \in \mathfrak{m}$. A recíproca vale se $H$ é conexo.

Lema 1.6.7 Existe uma correspondência biunívoca entre as métricas semiRiemannianas $G$-invariantes em $G / H$ e os produtos escalares $\overline{\operatorname{Ad}}(H)$-invariantes $\langle\cdot, \cdot\rangle$ em $\mathfrak{m}$. A $\overline{\operatorname{Ad}}(H)$-invariância de $\langle\cdot, \cdot\rangle$ implica

$$
\left\langle\operatorname{pr}_{\mathfrak{m}}([X, Y]), Z\right\rangle=-\left\langle Y, \operatorname{pr}_{\mathfrak{m}}([X, Z])\right\rangle,
$$

para quaisquer $X \in \mathfrak{h}$ e $Y, Z \in \mathfrak{m}$. A recíproca vale se $H$ é conexo.

Demonstração. Seja $\langle\cdot, \cdot\rangle$ um produto escalar $\overline{\operatorname{Ad}}(H)$-invariante em $\mathfrak{m}$. Exigindo-se que o isomorfismo $\left.\mathrm{d} \pi(1)\right|_{\mathfrak{m}}: \mathfrak{m} \rightarrow T_{1 H}(G / H)$ seja uma isometria, determinamos um produto escalar $\langle\cdot, \cdot\rangle_{1 H}$ em $T_{1 H}(G / H)$. O fato que $\langle\cdot, \cdot \cdot\rangle$ é $\overline{\operatorname{Ad}}(H)$-invariante significa que o isomorfismo $\overline{\mathrm{Ad}}_{h}$ é uma isometria, para todo $h \in H$. Porém, (1.56) implica que $\mathrm{d} \bar{L}_{h}(1 H)$ é uma isometria linear, para todo $h \in H$. Usaremos este fato para estender $\langle\cdot, \cdot\rangle_{1 H}$ a todo espaço $G / H$ de maneira $G$-invariante. Sejam $g_{1}, g_{2} \in G$ tais que $\bar{L}_{g_{1}}(1 H)=x=\bar{L}_{g_{2}}(1 H)$. Mostremos que o pull-back de $\langle\cdot, \cdot\rangle_{1 H}$ pelos isomorfismos

$$
\mathrm{d} \bar{L}_{g_{1}^{-1}}(1 H), \mathrm{d} \bar{L}_{g_{2}^{-1}}(1 H): T_{x}(G / H) \rightarrow T_{1 H}(G / H)
$$

define o mesmo produto escalar $\langle\cdot, \cdot\rangle_{x}$ em $T_{x}(G / H)$. De fato, $\bar{L}_{g_{1}}(1 H)=$ $\bar{L}_{g_{2}}(1 H)$ significa que $g_{1} H=g_{2} H$, ou seja, $g_{2}^{-1} g_{1}=h \in H$. Temos $\bar{L}_{h}=$ $\bar{L}_{g_{2}^{-1}} \circ \bar{L}_{g_{1}}$. Assim, dados $X, Y \in T_{x}(G / H)$, temos:

$$
\begin{aligned}
\left\langle\mathrm{d} \bar{L}_{g_{1}^{-1}}(1 H) \cdot X\right. & \left.\mathrm{d} \bar{L}_{g_{1}^{-1}}(1 H) \cdot Y\right\rangle \\
& =\left\langle\mathrm{d} \bar{L}_{h}(1 H) \mathrm{d} \bar{L}_{g_{1}^{-1}}(1 H) \cdot X, \mathrm{~d} \bar{L}_{h}(1 H) \mathrm{d} \bar{L}_{g_{1}^{-1}}(1 H) \cdot Y\right\rangle \\
& =\left\langle\mathrm{d} \bar{L}_{g_{2}^{-1}}(1 H) \cdot X, \mathrm{~d} \bar{L}_{g_{2}^{-1}}(1 H) \cdot Y\right\rangle .
\end{aligned}
$$

A métrica $\langle\cdot, \cdot\rangle$ em $G / H$, assim obtida, claramente é $G$-invariante. A diferenciabilidade de $\langle\cdot, \cdot\rangle$ segue da existência de seções locais (ver Observação 1.1.7). Reciprocamente, seja $\langle\cdot, \cdot\rangle$ uma métrica $G$-invariante em $G / H$. Disso decorre que os isomorfismos $\mathrm{d} \bar{L}_{h}(1 H)$ são isometrias lineares, para todo $h \in H$. Exigindo-se que $\left.\mathrm{d} \pi(1)\right|_{\mathfrak{m}}: \mathfrak{m} \rightarrow T_{1 H}(G / H)$ seja uma isometria e usando (1.56), o pull-back de $\langle\cdot, \cdot\rangle_{1 H}$ por $\left.\mathrm{d} \pi(1)\right|_{\mathfrak{m}}$ determina um produto escalar $\overline{\operatorname{Ad}}(H)$-invariante em $\mathfrak{m}$. A segunda parte do Lema segue diretamente do Corolário 1.6.6. 
Observação 1.6.8 O Lema 1.6.7 é um caso particular de uma correspondência biunívoca natural entre os tensores $G$-invariantes do tipo $(r, s)$ em $G / H$ e os tensores $\overline{\operatorname{Ad}}(H)$-invariantes do tipo $(r, s)$ em $\mathfrak{m}$. 


\section{Capítulo 2}

\section{Espaços Fibrados e Conexões}

\subsection{Fibrados principais}

Sejam $M$ uma variedade diferenciável, $G$ um grupo de Lie, $P$ um conjunto e $\Pi: P \rightarrow M$ uma aplicação. Para cada $x \in M$, denotemos por $P_{x}$ o subconjunto $\Pi^{-1}(x)$ de $P$, chamado a fibra de $P$ sobre $x$. Assumimos que, para cada $x \in M$, temos uma ação à direita livre e transitiva do grupo $G$ na fibra $P_{x}$. Note que isso é equivalente a assumir que $\Pi$ é sobrejetora e que é dado uma ação à direita

$$
(p, g) \in P \times G \mapsto p \cdot g \in P
$$

de $G$ em $P$ tal que $\Pi(p \cdot g)=\Pi(p)$, para quaisquer $p \in P$ e $g \in G$, e tal que, para quaisquer $p, q \in P$, com $\Pi(p)=\Pi(q)$, existe um único $g \in G$ tal que $p \cdot g=q$.

Uma seção local de $\Pi$ é uma aplicação $s: U \rightarrow P$, onde $U \subset M$ é um subconjunto aberto, tal que $\Pi \circ s$ é a aplicação de inclusão. Dados duas seções locais $s_{1}: U_{1} \rightarrow P$ e $s_{2}: U_{2} \rightarrow P$, existe uma única aplicação

$$
g: U_{1} \cap U_{2} \rightarrow G
$$

tal que $s_{2}(x)=s_{1}(x) \cdot g(x)$, para todo $x \in U_{1} \cap U_{2}$; a aplicação (2.2) é chamada a aplicação de transição de $s_{1}$ para $s_{2}$. As seções locais $s_{1}$ e $s_{2}$ são chamadas seções locais compativeis se a aplicação (2.2) é diferenciável.

Um atlas de seções locais de $\Pi$ é um conjunto $\mathcal{A}$ de seções locais de $\Pi$, duas a duas compatíveis, cujos domínios formam uma cobertura do conjunto $M$. Qualquer atlas $\mathcal{A}$ de seções locais de $\Pi$ está contido em um único atlas maximal $\mathcal{A}_{\max }$ de seções locais de $\Pi$. 
Definição 2.1.1 Um G-fibrado principal consiste dos seguintes objetos: um conjunto $P$, chamado o espaço total; uma variedade diferenciável $M$, chamada o espaço base; uma aplicação $\Pi: P \rightarrow M$, chamada a projeção; um grupo de Lie $G$, chamado o grupo estrutural; uma ação à direita livre e transitiva do grupo $G$ em cada fibra $P_{x}$ e um atlas maximal $\mathcal{A}_{\max }$ de seções locais de $\Pi$.

Um $G$-fibrado principal será denotado por $P(M, \Pi, G)$. Caso não exista perigo de confusão, denotaremos o $G$-fibrado principal simplesmente por $P$ e diremos apenas fibrado principal, subentendendo todos os dados descritos na Definição 2.1.1.

Seja $P(M, \Pi, G)$ um $G$-fibrado principal. Dado uma seção local $s: U \rightarrow$ $P$ de $P$, a aplicação

$$
\beta_{s}: U \times G \rightarrow \Pi^{-1}(U) \subset P,
$$

definida por

$$
\beta_{s}(x, g)=s(x) \cdot g,
$$

para quaisquer $x \in U$ e $g \in G$, é uma bijeção. Além disso, se $s_{1}: U_{1} \rightarrow P$ e $s_{2}: U_{2} \rightarrow P$ são seções locais de $P$ pertencentes a $\mathcal{A}_{\max }$, então a aplicação de transição de $\beta_{s_{1}}^{-1}$ para $\beta_{s_{2}}^{-1}$,

$$
\beta_{s_{2}}^{-1} \circ \beta_{s_{1}}: \beta_{s_{1}}^{-1}\left(\Pi^{-1}\left(U_{1} \cap U_{2}\right)\right) \rightarrow \beta_{s_{2}}^{-1}\left(\Pi^{-1}\left(U_{1} \cap U_{2}\right)\right),
$$

é dada por

$$
\left(\beta_{s_{2}}^{-1} \circ \beta_{s_{1}}\right)(x, h)=(x, g(x) \cdot h),
$$

para quaisquer $x \in U_{1} \cap U_{2}$ e $h \in G$, onde $g: U_{1} \cap U_{2} \rightarrow G$ é a aplicação de transição de $s_{2}$ para $s_{1}$. De $(2.4)$ segue que $\beta_{s_{2}}^{-1} \circ \beta_{s_{1}}$ é diferenciável e, pelo Lema 1.1.2, existe uma única estrutura diferenciável no conjunto $P$ tal que, para toda seção local $s: U \rightarrow P$ pertencente a $\mathcal{A}_{\max }$, o conjunto $\Pi^{-1}(U)$ é aberto em $P$ e a aplicação $\beta_{s}$, definida em (2.3), é um difeomorfismo diferenciável. O fato de que as topologias de $M$ e $G$ são Hausdorff e satisfazem o segundo axioma da enumerabilidade implicam que a topologia de $P$ é também Hausdorff e segundo enumerável. Portanto, $P$ é uma variedade diferenciável.

Além disso, temos a seguinte: 
Proposição 2.1.2 Seja $P(M, \Pi, G)$ um fibrado principal, onde $P$ está munido da estrutura diferenciável descrita acima. Então, a ação à direita (2.1) é diferenciável; a projeção $\Pi: P \rightarrow M$ é uma submersão diferenciável; para todo $x \in M$, a fibra $P_{x}$ é uma subvariedade mergulhada de $P$; para quaisquer $x \in M$ e $p \in P_{x}$, a aplicação $\beta_{p}: G \rightarrow P_{x}$ (cf. (1.38)) é um difeomorfismo diferenciável; toda seção local $s: U \rightarrow P$ pertencente a $\mathcal{A}_{\text {max }}$ é uma aplicação diferenciável e, reciprocamente, toda seção local diferenciável $s: U \rightarrow P$ pertence a $\mathcal{A}_{\max }$.

Exemplo 2.1.3 Sejam $M$ uma variedade diferenciável, $G$ um grupo de Lie e $P_{0}$ um conjunto. Suponha que $G$ age à direita no conjunto $P_{0}$ de forma livre e transitiva. Definimos $P=M \times P_{0}$. Denotemos por $\Pi: P \rightarrow M$ a projeção sobre o primeiro fator e definimos uma ação à direita de $G$ em $P$ por

$$
(x, p) \cdot g=(x, p \cdot g),
$$

para quaisquer $x \in M, p \in P_{0}$ e $g \in G$. Para todo $p \in P_{0}$, a aplicação $s^{p}: M \rightarrow P$, definida por $s^{p}(x)=(x, p)$, é uma seção global de $\Pi$ e o conjunto $\left\{s^{p}: p \in P_{0}\right\}$ é um atlas de seções de $\Pi$. Assim, $P$ é um $G$-fibrado principal, chamado o fibrado principal trivial. A estrutura diferenciável de $P=M \times P_{0}$, como fibrado principal, coincide com a estrutura diferenciável canônica dada pelo produto de variedades diferenciáveis.

Exemplo 2.1.4 Seja $P(M, \Pi, G)$ um fibrado principal. Dado um subconjunto aberto $U \subset M$, denotemos por

$$
\left.P\right|_{U}=\Pi^{-1}(U) \subset P .
$$

A ação à direita de $G$ em $P$ restringe-se a uma ação à direita de $G$ em $\left.P\right|_{U}$. Além disso, a projeção $\Pi$ restringe-se a uma aplicação (que também denotaremos por $\Pi$ ) de $\left.P\right|_{U}$ em $U$. O conjunto $\left.P\right|_{U}$ é então um $G$-fibrado principal sobre a variedade diferenciável $U$, cujo atlas maximal de seções locais consiste de todas as seções locais diferenciáveis de $P$ com domínio contido em $U ;\left.P\right|_{U}$ é chamado a restrição de $P$ ao aberto $U$. Claramente $\left.P\right|_{U}$ é um subconjunto aberto de $P$ e a estrutura diferenciável de $\left.P\right|_{U}$, como fibrado principal, coincide com a estrutura diferenciável induzida por $P$.

Exemplo 2.1.5 Sejam $G$ um grupo de Lie e $H$ um subgrupo fechado de $G$. Consideremos a aplicação quociente $\Pi: G \rightarrow G / H$ e a ação de $H$ em $G$ por translações à direita. Para cada $x \in G / H$, a fibra $\Pi^{-1}(x)$ é uma classe lateral à esquerda de $H$ em $G$, logo a ação de $H$ em $\Pi^{-1}(x)$ é livre e 
transitiva. Se $s_{1}: U_{1} \rightarrow G$ e $s_{2}: U_{2} \rightarrow G$ são seções locais diferenciáveis de $\Pi$ então a aplicação de transição $h: U_{1} \cap U_{2} \rightarrow H$ é dada por:

$$
h(x)=s_{1}(x)^{-1} \cdot s_{2}(x),
$$

para todo $x \in U_{1} \cap U_{2}$ e, portanto, é diferenciável. Assim, o conjunto de todas as seções locais diferenciáveis de $\Pi$ é um atlas de seções locais de $\Pi$ e $\Pi: G \rightarrow G / H$ é um $H$-fibrado principal munido com o atlas de todas as seções locais diferenciáveis de П. Além disso, a estrutura diferenciável em $G$ induzida por tal atlas coincide com a estrutura diferenciável original de $G$.

\subsubsection{Subfibrados principais}

Seja $P(M, \Pi, G)$ um fibrado principal e consideremos um subgrupo de Lie $H$ de $G$.

Definição 2.1.6 Um $H$-subfibrado principal de $P$ é um suconjunto $Q$ de $P$ tal que, para todo $x \in M, Q_{x}=P_{x} \cap Q$ é uma $H$-órbita e existe uma seção local diferenciável $s: U \rightarrow P$ de $P$, com $x \in U$ e $s(U) \subset Q$.

A ação à direita de $G$ em $P$ restringe-se a uma ação à direita de $H$ em $Q$. Considerando a restrição da projeção $\Pi: P \rightarrow M$ a $Q$ (que também será denotada por $\Pi$ ), segue que $Q$ é um $H$-fibrado principal sobre $M$, cujo atlas maximal consiste de todas as seções locais $s: U \rightarrow Q$ de $Q$ tais que $i \circ s: U \rightarrow P$ é uma seção local diferenciável de $P$, onde $i: Q \rightarrow P$ denota a aplicação de inclusão ${ }^{1}$.

Um $H$-subfibrado principal de $P$ será denotado por $Q(M, \Pi, H)$. Não havendo perigo de confusão, o subfibrado principal será denotado simplesmente por $Q$.

Seja $Q$ um subfibrado principal de $P$. O conjunto $Q$, sendo o espaço total de um fibrado principal, tem uma estrutura diferenciável. Analisemos a relação entre as estruturas diferenciáveis de $Q$ e de $P$. Se $s: U \rightarrow Q$ é uma seção local diferenciável de $Q$ então $i \circ s: U \rightarrow P$ é uma seção local diferenciável de $P$. Temos, assim, o seguinte diagrama comutativo:

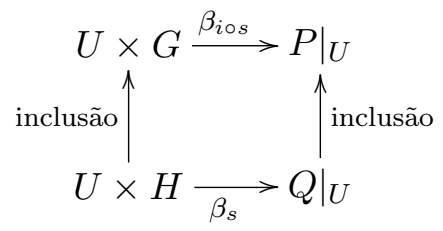

\footnotetext{
${ }^{1}$ A compatibilidade entre seções locais de $Q$ segue do Lema 1.1.3 e da Observação 1.1.5.
} 
Como as flechas horizontais são difeomorfismos diferenciáveis segue que a aplicação inclusão $i: Q \rightarrow P$ é uma imersão ${ }^{2}$ diferenciável e, portanto, $Q$ é uma subvariedade imersa de $P$.

Seja $P(M, \Pi, G)$ um fibrado principal. Dados $x \in M$ e $p \in P_{x}$, o espaço tangente $T_{p} P_{x}$ é um subespaço de $T_{p} P$, chamado o espaço vertical de $P$ no ponto $p$. Denotemos

$$
\operatorname{Ver}_{p}(P)=T_{p} P_{x}
$$

Claramente, $\operatorname{Ver}_{p}(P)=\operatorname{Ker}\left(\mathrm{d} \Pi_{p}\right)$. Como a aplicação $\beta_{p}: G \rightarrow P_{x}$ é um difeomorfismo diferenciável, sua diferencial no elemento identidade $1 \in G$ é um isomorfismo

$$
\mathrm{d} \beta_{p}(1): \mathfrak{g} \rightarrow \operatorname{Ver}_{p}(P) .
$$

O isomorfismo (2.5) é chamado o isomorfismo canônico de $\mathfrak{g}$ em $\operatorname{Ver}_{p}(P)$.

Diferenciando a ação à direita (2.1) em relação a primeira variável, obtemos uma ação à direita de $G$ no fibrado tangente $T P$. De forma mais explícita, para quaisquer $g \in G$ e $v \in T P$, temos:

$$
v \cdot g=d \gamma_{g}(v) \in T P,
$$

onde $\gamma_{g}: P \rightarrow P$ é o difeomorfismo diferenciável dado pela ação de $g$ em $P$ (cf. (1.39)). Como $\gamma_{g}: P \rightarrow P$ transforma fibras em fibras, a ação de $G \mathrm{em}$ $T P$ transforma espaços verticais em espaços verticais, ou seja,

$$
\mathrm{d} \gamma_{g}\left(\operatorname{Ver}_{p}(P)\right)=\operatorname{Ver}_{p \cdot g}(P),
$$

para quaisquer $p \in P$ e $g \in G$. Temos, assim, o seguinte diagrama comutativo:

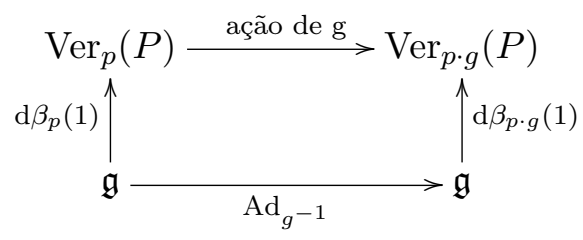

onde $\operatorname{Ad}: G \rightarrow \mathrm{GL}(\mathfrak{g})$ denota a representação adjunta de $G$ em $\mathfrak{g}$ definida em (1.48).

\footnotetext{
${ }^{2} \mathrm{~A}$ aplicação de inclusão $i: Q \rightarrow P$ é um mergulho diferenciável se, e somente se, $H$ é um subgrupo de Lie mergulhado de $G$ (cf. Seção 1.3).
} 


\subsubsection{Morfismos de fibrados principais}

Sejam $P, Q$ fibrados principais sobre uma mesma variedade diferenciável $M$ e com grupos estruturais $G$ e $H$, respectivamente. Um morfismo de fibrados principais é uma aplicação diferenciável $\phi: P \rightarrow Q$, satisfazendo $\phi\left(P_{x}\right) \subset Q_{x}$, para todo $x \in M$, de modo que existe um homomorfismo de grupos $\phi_{0}: G \rightarrow H$ tal que, para todo $x \in M$, a aplicação $\phi_{x}=\left.\phi\right|_{P_{x}}: P_{x} \rightarrow$ $Q_{x}$ satisfaz:

$$
\phi_{x}(p \cdot g)=\phi_{x}(p) \cdot \phi_{0}(g),
$$

para quaisquer $p \in P_{x}$ e $g \in G$.

O homomorfismo $\phi_{0}: G \rightarrow H$ é unicamente determinado pelo morfismo de fibrados principais $\phi: P \rightarrow Q$. Fixados $x \in M$ e $p \in P_{x}$, a comutatividade do diagrama

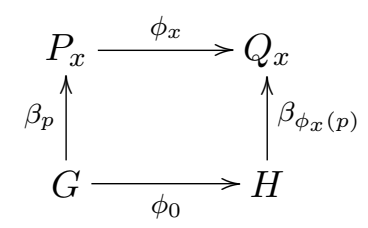

mostra que a diferenciabilidade de $\phi$ implica na diferenciabilidade de $\phi_{0}$. Assim, $\phi_{0}$ é de fato um homomorfismo de grupos de Lie, chamado o homomorfismo subjacente de $\phi$.

A composição $\phi \circ \psi$ dos morfismos de fibrados principais $\phi$ e $\psi$ com homomorfismos subjacentes $\phi_{0}$ e $\psi_{0}$, respectivamente, é um morfismo de fibrados principais com homomorfismo subjacente $\phi_{0} \circ \psi_{0}$. Um morfismo de fibrados principais $\phi$ é bijetor se, e somente se, seu homomorfismo subjacente $\phi_{0}$ é bijetor. Um morfismo bijetor de fibrados principais é chamado um isomorfismo de fibrados principais. Se $\phi$ é um isomorfismo de fibrados principais com homomorfismo subjacente $\phi_{0}$ então $\phi$ é um difeomorfismo diferenciável e $\phi^{-1}$ é também um isomorfismo de fibrados principais com homomorfismo subjacente $\phi_{0}^{-1}$.

Exemplo 2.1.7 Se $Q(M, \Pi, H)$ é um subfibrado principal de $P(M, \Pi, G)$ então a aplicação inclusão $i: Q \rightarrow P$ é um morfismo de fibrados principais cujo homomorfismo subjacente é a aplicação inclusão de $H$ em $G$.

Lema 2.1.8 Sejam $P(M, \Pi, G)$ um fibrado principal, $Q$ um conjunto, $\Pi^{\prime}$ : $Q \rightarrow M$ uma aplicação, $H$ um grupo de Lie e assuma que é dado uma ação diferenciável à direita de $H$ em cada fibra $Q_{x}$ de forma livre e transitiva. Sejam $\phi_{0}: G \rightarrow H$ um homomorfismo de grupos de Lie e $\phi: P \rightarrow Q$ uma 
aplicação tal que $\phi\left(P_{x}\right) \subset Q_{x}$ e $\phi_{x}(p \cdot g)=\phi_{x}(p) \cdot \phi_{0}(g)$, para quaisquer $x \in M, p \in P_{x}$ e $g \in G$. Então, existe um único atlas maximal de seções locais de $\Pi^{\prime}$ que torna $\phi: P \rightarrow Q$ um morfismo de fibrados principais.

Demonstração. Consideremos o seguinte conjunto de seções locais de $\Pi^{\prime}$ :

$$
\mathcal{A}=\{\phi \circ s: s \text { é seção local diferenciável de } P\} .
$$

Mostremos que $\mathcal{A}$ é um atlas de seções locais de $\Pi^{\prime}$. Claramente os domínios das seções locais de $\mathcal{A}$ formam uma cobertura de $M$. Além disso, se $s_{1}$ : $U_{1} \rightarrow P$ e $s_{2}: U_{2} \rightarrow P$ são seções locais diferenciáveis de $P$ com aplicação de transição $g: U_{1} \cap U_{2} \rightarrow G$, então a aplicação de transição de $\phi \circ s_{1}$ para $\phi \circ s_{2}$ é $\phi_{0} \circ g: U_{1} \cap U_{2} \rightarrow H$. Assim, $\phi \circ s_{1}$ e $\phi \circ s_{2}$ são compatíveis e $\mathcal{A}$ é um atlas de seções locais de $\Pi^{\prime}$. Finalmente, observe que um atlas maximal $\mathcal{A}_{\max }$ de seções locais de $\Pi^{\prime}$ torna $\phi: P \rightarrow Q$ um morfismo de fibrados principais se, e somente se, $\mathcal{A}_{\max }$ é o atlas maximal de seções locais de $\Pi^{\prime}$ contendo $\mathcal{A}$.

\subsubsection{Pull-back de fibrados principais}

Sejam $P(M, \Pi, G)$ um fibrado principal, $N$ uma variedade diferenciável e $f: N \rightarrow M$ uma aplicação diferenciável. O pull-back de $P$ por $f$ é o conjunto $f^{*} P$ definido por

$$
f^{*} P=\bigcup_{y \in N}\left(\{y\} \times P_{f(y)}\right) .
$$

A restrição a $f^{*} P$ da projeção sobre o primeiro fator define uma aplicação $\Pi_{1}: f^{*} P \rightarrow N$ e a restrição a $f^{*} P$ da projeção sobre o segundo fator define uma aplicação $\bar{f}: f^{*} P \rightarrow P$, de modo que o diagrama seguinte comuta:

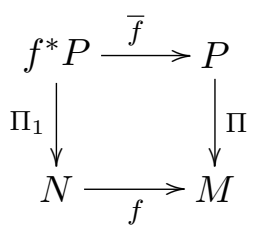

O pull-back $f^{*} P$ é precisamente o subconjunto de $N \times P$ onde as aplicações $\Pi \circ \bar{f}$ e $f \circ \Pi_{1}$ coincidem. Além disso, a aplicação $\left(\Pi_{1}, \bar{f}\right): f^{*} P \rightarrow N \times P$ é simplesmente a aplicação de inclusão. Isso motiva o seguinte:

Lema 2.1.9 Sejam $P(M, \Pi, G)$ um fibrado principal, $N$ uma variedade diferenciável e $f: N \rightarrow M$ uma aplicação diferenciável. Dados um conjunto $X$ e aplicações $\tau_{1}: X \rightarrow N, \tau_{2}: X \rightarrow P$ com $\Pi \circ \tau_{2}=f \circ \tau_{1}$, existe uma única aplicação $\tau: X \rightarrow f^{*} P$ tal que $\Pi_{1} \circ \tau=\tau_{1}$ e $\bar{f} \circ \tau=\tau_{2}$. 
Demonstração. A hipótese $\Pi \circ \tau_{2}=f \circ \tau_{1}$ significa que a imagem da aplicação $\left(\tau_{1}, \tau_{2}\right): X \rightarrow N \times P$ está contida em $f^{*} P$. Como $\left(\Pi_{1}, \bar{f}\right): f^{*} P \rightarrow$ $N \times P$ é a aplicação de inclusão, existe uma única aplicação $\tau: X \rightarrow f^{*} P$ tal que

$$
\left(\Pi_{1}, \bar{f}\right) \circ \tau=\left(\tau_{1}, \tau_{2}\right) .
$$

Porém, a igualdade em (2.8) é equivalente a $\Pi_{1} \circ \tau=\tau_{1}$ e $\bar{f} \circ \tau=\tau_{2}$.

Mostraremos agora que $f^{*} P$ é um $G$-fibrado principal sobre $N$. Para cada $y \in N$, identifiquemos a fibra $\left(f^{*} P\right)_{y}=\{y\} \times P_{f(y)}$ de $f^{*} P$ com a fibra $P_{f(y)}$ de $P$. Sob esta identificação, o grupo $G$ age à direita em cada fibra de $f^{*} P$ de forma livre e transitiva. O próximo passo é definir um atlas de seções locais de $\Pi_{1}: f^{*} P \rightarrow N$.

Definição 2.1.10 Uma seção local de $P$ ao longo de $f$ é uma aplicação $\sigma: V \rightarrow P$, definida em um subconjunto aberto $V$ de $N$, tal que $\Pi \circ \sigma=\left.f\right|_{V}$.

Se $s: U \rightarrow P$ é uma seção local de $P$ então a composta $s \circ f: f^{-1}(U) \rightarrow P$ é uma seção local de $P$ ao longo de $f$. Além disso, se $\bar{\sigma}: V \rightarrow f^{*} P$ é uma seção local de $\Pi_{1}: f^{*} P \rightarrow N$, então $\bar{f} \circ \bar{\sigma}: V \rightarrow P$ é uma seção local de $P$ ao longo de $f$. Por outro lado, se $\sigma: V \rightarrow P$ é uma seção local de $P$ ao longo de $f$, existe uma única seção local $\bar{\sigma}: V \rightarrow f^{*} P$ de $\Pi_{1}: f^{*} P \rightarrow N$ tal que $\bar{f} \circ \bar{\sigma}=\sigma$. De fato, tomando $X=V, \tau_{1}: V \rightarrow N$ a aplicação de inclusão e $\tau_{2}=\sigma$ então $\bar{\sigma}$ é a aplicação $\sigma$ dada pelo Lema 2.1.9. O seguinte diagrama comutativo ilustra a relação entre $\sigma$ e $\bar{\sigma}$.

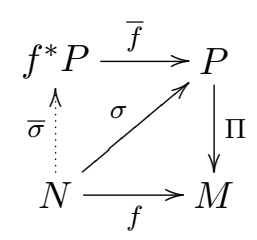

Seja então $s: U \rightarrow P$ uma seção local diferenciável de $P$. Defina

$$
\sigma=s \circ f: f^{-1}(U) \rightarrow P
$$

e considere

$$
\bar{\sigma}: f^{-1}(U) \rightarrow f^{*} P
$$

a única seção local de $P$ tal que $\bar{f} \circ \bar{\sigma}=\sigma$. Mostremos que o conjunto

$$
\mathcal{A}=\{\bar{\sigma}: \sigma=s \circ f \text { e } s \text { é uma seção local diferenciável de } P\}
$$


é um atlas de seções locais de $\Pi_{1}: f^{*} P \rightarrow N$. De fato, sejam $s_{1}: U_{1} \rightarrow P$ e $s_{2}: U_{2} \rightarrow P$ seções locais diferenciáveis de $P$ com aplicação de transição $g: U_{1} \cap U_{2} \rightarrow G$. Defina $\sigma_{i}=s_{i} \circ f, i=1,2$, e considere as seções locais $\bar{\sigma}_{i}: f^{-1}\left(U_{i}\right) \rightarrow f^{*} P$ de $\Pi_{1}: f^{*} P \rightarrow N$ tais que $\bar{f} \circ \bar{\sigma}_{i}=\sigma_{i}, i=1,2$. A aplicação de transição de $\bar{\sigma}_{1}$ para $\bar{\sigma}_{2}$ é a aplicação

$$
g \circ f: f^{-1}\left(U_{1} \cap U_{2}\right) \rightarrow G,
$$

o que mostra que as seções locais $\bar{\sigma}_{1}$ e $\bar{\sigma}_{2}$ são compatíveis. Isso implica que o conjunto (2.12) é um atlas de seções locais de $f^{*} P$. Considerando $f^{*} P$ com o único atlas maximal de seções locais contendo $(2.12), f^{*} P$ torna-se de fato um $G$-fibrado principal sobre $N$.

Exemplo 2.1.11 Sejam $P(M, \Pi, G)$ um fibrado principal, $Q$ um $H$-subfibrado principal de $P$ e $f: N \rightarrow M$ uma aplicação diferenciável. Então, $f^{*} Q$ é um $H$-subfibrado principal de $f^{*} P$. De fato, como cada fibra $\left(f^{*} Q\right)_{y}$ de $f^{*} Q$ está identificada com a fibra $Q_{f(y)}$ de $Q$, segue que $\left(f^{*} Q\right)_{y}$ é uma $H$ órbita. Além disso, dado $y \in N$, seja $s: U \rightarrow P$ uma seção local diferenciável de $P$, com $f(y) \in U$ e $s(U) \subset Q$. Definindo $\bar{\sigma}: f^{-1}(U) \rightarrow f^{*} P$, como em (2.11), temos que $y \in f^{-1}(U)$ e $\bar{\sigma}\left(f^{-1}(U)\right) \subset f^{*} Q$. Portanto, $f^{*} Q$ é um $H$-subfibrado principal de $f^{*} P$.

\section{$2.2 \quad$ Fibrados vetoriais}

Sejam $E$ um conjunto, $M$ uma variedade diferenciável e $\pi: E \rightarrow M$ uma aplicação. Para cada $x \in M$, denotemos por $E_{x}$ o subconjunto $\pi^{-1}(x)$ de $E$, chamado a fibra de $E$ sobre $x$. Assumimos que, para cada $x \in M$, a fibra $E_{x}$ tem uma estrutura de espaço vetorial real isomorfa a $\mathbb{R}^{n}$.

Dado $x \in M$, denotemos por $\mathrm{FR}\left(E_{x}\right)$ o conjunto de todos os isomorfismos lineares de $\mathbb{R}^{n}$ em $E_{x}$ (também chamados de referenciais de $E_{x}$ ). Note que o grupo de Lie $\operatorname{GL}\left(\mathbb{R}^{n}\right)$ age à direita $\operatorname{em~} \mathrm{FR}\left(E_{x}\right)$ de forma livre e transitiva. Definimos

$$
\operatorname{FR}(E)=\bigcup_{x \in M} \operatorname{FR}\left(E_{x}\right)
$$

e consideremos a aplicação $\Pi: \operatorname{FR}(E) \rightarrow M$ que transforma $\operatorname{FR}\left(E_{x}\right)$ sobre $x$, para todo $x \in M$.

Definição 2.2.1 Um fibrado vetorial consiste dos seguintes objetos: um conjunto $E$, chamado o espaço total; uma variedade diferenciável $M$, chamada o espaço base; uma aplicação $\pi: E \rightarrow M$, chamada a projeção; uma 
estrutura de espaço vetorial real em cada fibra $E_{x}$ tal que $E_{x}$ e $\mathbb{R}^{n}$ sejam espaços vetoriais reais isomorfos; um atlas maximal $\mathcal{A}_{\text {max }}$ de seções locais de $\Pi: \operatorname{FR}(E) \rightarrow M$.

O atlas maximal $\mathcal{A}_{\max }$ torna $\Pi: \mathrm{FR}(E) \rightarrow M$ um $\mathrm{GL}\left(\mathbb{R}^{n}\right)$-fibrado principal, chamado o fibrado principal dos referenciais de E. O espaço Euclidiano $\mathbb{R}^{n}$ é chamado a fibra típica do fibrado vetorial. Um fibrado vetorial será denotado por $\left(E, \pi, M, \mathbb{R}^{n}\right)$. Caso não exista perigo de confusão, denotaremos o fibrado vetorial simplesmente por $E$, subentendendo todos os dados descritos na Definição 2.2.1.

Definiremos agora uma estrutura diferenciável no espaço total de um fibrado vetorial $E$. Toda seção local diferenciável $s: U \rightarrow \mathrm{FR}(E)$ define uma bijeção

$$
\beta_{s}: U \times \mathbb{R}^{n} \rightarrow \pi^{-1}(U) \subset E,
$$

dada por

$$
\beta_{s}(x, v)=s(x) \cdot v,
$$

para quaisquer $x \in U$ e $v \in \mathbb{R}^{n}$. A aplicação $\beta_{s}$, definida em (2.13), é chamada a trivialização local de $E$ correspondente à seção local $s$.

Sejam $s_{1}: U_{1} \rightarrow \mathrm{FR}(E)$ e $s_{2}: U_{2} \rightarrow \mathrm{FR}(E)$ seções locais diferenciáveis de $\operatorname{FR}(E)$. Então, a aplicação de transição de $\beta_{s_{1}}^{-1}$ para $\beta_{s_{2}}^{-1}$,

$$
\beta_{s_{2}}^{-1} \circ \beta_{s_{1}}: \beta_{s_{1}}^{-1}\left(\pi^{-1}\left(U_{1} \cap U_{2}\right)\right) \rightarrow \beta_{s_{2}}^{-1}\left(\pi^{-1}\left(U_{1} \cap U_{2}\right)\right)
$$

é dada por

$$
\left(\beta_{s_{2}}^{-1} \circ \beta_{s_{1}}\right)(x, v)=(x, g(x) \cdot v),
$$

para quaisquer $x \in U_{1} \cap U_{2}$ e $v \in \mathbb{R}^{n}$, onde $g: U_{1} \cap U_{2} \rightarrow \mathrm{GL}\left(\mathbb{R}^{n}\right)$ é a aplicação de transição de $s_{2}$ para $s_{1}$. Assim, $\beta_{s_{2}}^{-1} \circ \beta_{s_{1}}$ é diferenciável e, pelo Lema 1.1.2 existe uma única estrutura diferenciável no conjunto $E$ tal que, para toda seção local diferenciável $s: U \rightarrow \mathrm{FR}(E)$, o conjunto $\pi^{-1}(U)$ é aberto em $E$ e a aplicação $\beta_{s}$, dada em (2.13), é um difeomorfismo diferenciável ${ }^{3}$. Além disso, a topologia de $E$ é Hausdorff e satisfaz o segundo axioma da enumerabilidade e, portanto, $E$ é uma variedade diferenciável.

Além disso, temos a seguinte:

\footnotetext{
${ }^{3}$ Aqui, estamos considerando a fibra típica $\mathbb{R}^{n}$ munida de sua estrutura diferenciável canônica, induzida de sua estutura de espaço vetorial.
} 
Proposição 2.2.2 Dado um fibrado vetorial $\left(E, \pi, M, \mathbb{R}^{n}\right)$, valem as seguintes propriedades: a projeção $\pi: E \rightarrow M$ é uma submersão diferenciável; para todo $x \in M$, a fibra $E_{x}$ é uma subvariedade mergulhada de $E$; para todo $x \in M$, a estrutura diferenciável da fibra $E_{x}$, como subvariedade de $E$, coincide com a estrutura diferenciável induzida por sua estrutura de espaço vetorial real de dimensão finita.

Exemplo 2.2.3 Dado uma variedade diferenciável $M$, seja $E=M \times \mathbb{R}^{n} \mathrm{e}$ consideremos a aplicação $\pi: E \rightarrow M$ dada pela projeção sobre o primeiro fator. Para cada $x \in M$, identifiquemos a fibra $E_{x}=\{x\} \times \mathbb{R}^{n}$ com $\mathbb{R}^{n}$, de modo que $E_{x}$ tem a estrutura de espaço vetorial real induzida de $\mathbb{R}^{n} \mathrm{e}$

$$
\mathrm{FR}(E)=M \times \mathrm{GL}\left(\mathbb{R}^{n}\right) .
$$

O conjunto $\mathrm{FR}\left(M \times \mathbb{R}^{n}\right)$ é um GL( $\left.\mathbb{R}^{n}\right)$-fibrado principal (cf. Exemplo 2.1.3) e, portanto, $\left(E, \pi, M, \mathbb{R}^{n}\right)$ é um fibrado vetorial, chamado o fibrado vetorial trivial. Claramente, a estrutura diferenciável de $E=M \times \mathbb{R}^{n}$ coincide com a estrutura diferenciável canônica dada pelo produto de variedades diferenciáveis.

Exemplo 2.2.4 Sejam $\left(E, \pi, M, \mathbb{R}^{n}\right)$ um fibrado vetorial e $U \subset M$ um subconjunto aberto. Consideremos

$$
\left.E\right|_{U}=\pi^{-1}(U)
$$

A projeção $\pi: E \rightarrow M$ restringe-se a uma aplicação de $\left.E\right|_{U}$ em $M$ e, para cada $x \in U$, a fibra $E_{x}$ de $\left.E\right|_{U}$ sobre $x$ tem a estrutura de espaço vetorial real. Além disso, temos:

$$
\operatorname{FR}\left(\left.E\right|_{U}\right)=\left.\operatorname{FR}(E)\right|_{U},
$$

$\operatorname{assim} \operatorname{FR}\left(\left.E\right|_{U}\right)$ é um $\operatorname{GL}\left(\mathbb{R}^{n}\right)$-fibrado principal sobre a variedade diferenciável $U$ (cf. Exemplo 2.1.4). Portanto, $\left(\left.E\right|_{U}, \pi, M, \mathbb{R}^{n}\right)$ é um fibrado vetorial, chamado a restrição do fibrado vetorial $E$ sobre o aberto $U$. A estrutura diferenciável de $\left.E\right|_{U}$, como fibrado vetorial, coincide com a estrutura diferenciável que $\left.E\right|_{U}$ herda de $E$ como subconjunto aberto. 
Exemplo 2.2.5 Dado uma variedade diferenciável $M^{n}$, sejam

$$
T M=\bigcup_{x \in M} T_{x} M
$$

seu fibrado tangente e $\pi: T M \rightarrow M$ a projeção canônica. Para cada $x \in M$, a fibra $T_{x} M$ tem a estrutura de espaço vetorial real isomorfo a $\mathbb{R}^{n}$. Seja $\varphi: U \rightarrow \varphi(U)$ uma carta local de $M$. Para cada $x \in U$, a aplicação $\mathrm{d} \varphi_{x}^{-1}: \mathbb{R}^{n} \rightarrow T_{x} M$ é um isomorfismo linear e a aplicação $s^{\varphi}: U \rightarrow \operatorname{FR}(T M)$, dada por

$$
s_{x}^{\varphi}=d \varphi(x)^{-1},
$$

para todo $x \in U$, é uma seção local de $\Pi: \operatorname{FR}(T M) \rightarrow M$. Se $\psi: V \rightarrow \psi(V)$ é outra carta local de $M$ e se

$$
\psi \circ \varphi^{-1}: \varphi(U \cap V) \rightarrow \psi(U \cap V)
$$

denota a aplicação de transição de $\varphi$ para $\psi$, então a aplicação de transição de $s^{\psi}$ para $s^{\varphi}$ é dada por

$$
x \in U \cap V \mapsto \mathrm{d}\left(\psi \circ \varphi^{-1}\right)(\varphi(x)) \in \mathrm{GL}\left(\mathbb{R}^{n}\right) .
$$

Assim, o conjunto

$$
\mathcal{A}=\left\{s^{\varphi}: \varphi \text { é carta local de } M\right\}
$$

é um atlas de seções locais de $\Pi: \operatorname{FR}(T M) \rightarrow M$. Considerando $\operatorname{FR}(T M)$ com o único atlas maximal de seções locais de $\Pi: \operatorname{FR}(T M) \rightarrow M$ contendo (2.14), segue que $\left(T M, \pi, M, \mathbb{R}^{n}\right)$ é um fibrado vetorial.

Exemplo 2.2.6 Sejam $\left(E^{1}, \pi_{1}, M, \mathbb{R}^{n_{1}}\right)$ e $\left(E^{2}, \pi_{2}, M, \mathbb{R}^{n_{2}}\right)$ fibrados vetoriais sobre a mesma base $M$. Para cada $x \in M$, consideremos a soma direta $E_{x}=E_{x}^{1} \oplus E_{x}^{2}$, munida da estrutura de espaço vetorial real dada pela soma direta de $E_{x}^{1}$ e $E_{x}^{2}$, e denotemos

$$
E=\bigcup_{x \in M} E_{x}^{1} \oplus E_{x}^{2}
$$

Seja $\pi: E \rightarrow M$ a projeção canônica que transforma $E_{x}^{1} \oplus E_{x}^{2}$ sobre $x \in M$. Dado $x \in M$, sejam $s_{1}: U_{1} \rightarrow \mathrm{FR}\left(E^{1}\right)$ e $s_{2}: U_{2} \rightarrow \mathrm{FR}\left(E^{2}\right)$ seções locais diferenciáveis, com $x \in U=U_{1} \cap U_{2}$. Então, a aplicação $\bar{s}: U \rightarrow \operatorname{FR}(E)$, dada por

$$
\bar{s}(x)=\left(s_{1}(x), s_{2}(x)\right)
$$


para todo $x \in U$, é uma seção local de $\operatorname{FR}(E)$. Sejam $s_{1}^{i}: U_{1}^{i} \rightarrow \operatorname{FR}\left(E^{1}\right)$ e $s_{2}^{i}: U_{2}^{i} \rightarrow \mathrm{FR}\left(E^{2}\right)$ seções locais diferenciáveis, $i=1,2$. Denotemos por $g_{i}: U_{1}^{i} \cap U_{2}^{i} \rightarrow \mathrm{GL}\left(\mathbb{R}^{n_{i}}\right)$ a aplicação de transição de $s_{i}^{1}$ para $s_{i}^{2}, i=1,2$. Então, a aplicação de transição ${ }^{4}$ de $\bar{s}_{1}=s_{1}^{1} \oplus s_{2}^{1}$ para $\bar{s}_{2}=s_{1}^{2} \oplus s_{2}^{2}$ é dada por

$$
x \in V_{1} \cap V_{2} \mapsto\left(g_{1}(x), g_{2}(x)\right) \in \mathrm{GL}\left(\mathbb{R}^{n}\right),
$$

onde $V_{i}=U_{i}^{1} \cap U_{i}^{2}, i=1,2$, e $\mathbb{R}^{n}=\mathbb{R}^{n_{1}} \oplus \mathbb{R}^{n_{2}}$. Assim, o conjunto

$$
\mathcal{A}=\left\{\bar{s}=s_{1} \oplus s_{2}: s_{i} \text { é seção local diferenciável de } \operatorname{FR}\left(E^{i}\right)\right\}
$$

é um atlas de seções locais de $\Pi: \operatorname{FR}(E) \rightarrow M$. Considerando $\operatorname{FR}(E)$ com o único atlas maximal de seções locais de $\Pi: \mathrm{FR}(E) \rightarrow M$ contendo (2.15), segue que $\left(E, \pi, M, \mathbb{R}^{n}\right)$ é um fibrado vetorial, chamado a soma de Whitney dos fibrados vetoriais $E^{1}$ e $E^{2}$. Usualmente, $E$ é denotado por $E^{1} \oplus E^{2}$.

\subsubsection{Seções de fibrados vetoriais}

Seja $\left(E, \pi, M, \mathbb{R}^{n}\right)$ um fibrado vetorial.

Definição 2.2.7 Uma seção local de $E$ é uma aplicação $\epsilon: U \rightarrow E$, definida em um subconjunto aberto $U \subset M$, tal que $\pi \circ \epsilon$ é a aplicação de inclusão, ou seja, $\epsilon(x) \in E_{x}$, para todo $x \in U$.

Uma seção local $\epsilon: M \rightarrow E$ de $E$, globalmente definida, é chamada uma seção de $E$. Observe que uma seção local $\epsilon: U \rightarrow E$ de $E$ é o mesmo que uma seção do fibrado vetorial $\left.E\right|_{U}$. Denotaremos por $\boldsymbol{\Gamma}(E)$ o conjunto de todas as seções diferenciáveis de $E$. O conjunto $\boldsymbol{\Gamma}(E)$ é um espaço vetorial real com as operações naturais de soma e multiplicação por escalar. Além disso, $\boldsymbol{\Gamma}(E)$ é um módulo sobre o anel $\mathrm{C}^{\infty}(M)$ das funções reais diferenciáveis definidas em $M$.

Exemplo 2.2.8 Seja $M$ uma variedade diferenciável. Uma seção diferenciável do fibrado tangente $T M$ é o mesmo que um campo vetorial diferenciável em $M$.

Sejam $\epsilon: U \rightarrow E$ uma seção local de $E$ e $s: U \rightarrow \operatorname{FR}(E)$ uma seção local diferenciável de $\operatorname{FR}(E)$. A aplicação $\tilde{\epsilon}: U \rightarrow \mathbb{R}^{n}$, definida por

$$
\tilde{\epsilon}(x)=s(x)^{-1} \cdot \epsilon(x),
$$

\footnotetext{
${ }^{4}$ Aqui, estamos identificando (diagonalmente) o produto $\operatorname{GL}\left(\mathbb{R}^{n_{1}}\right) \times \operatorname{GL}\left(\mathbb{R}^{n_{2}}\right)$ com um subgrupo de Lie de $\operatorname{GL}\left(\mathbb{R}^{n}\right)$.
} 
para todo $x \in U$, é chamada a representação da seção $\epsilon$ em relação a $s$. Se $\beta_{s}$ é a trivialização local de $E$ correspondente a $s$, temos:

$$
\epsilon(x)=\beta_{s}(x, \tilde{\epsilon}(x)),
$$

para todo $x \in U$. Logo, $\epsilon$ é diferenciável se, e somente se, $\tilde{\epsilon}$ é diferenciável.

Lema 2.2.9 Sejam $\left(E, \pi, M, \mathbb{R}^{n}\right)$ um fibrado vetorial e $x \in M$. Então:

(a) Para cada $p \in E_{x}$, existe uma seção $\epsilon \in \boldsymbol{\Gamma}(E)$, com $\epsilon(x)=p$.

(b) Dado uma seção local diferenciável $\epsilon: U \rightarrow E$, com $x \in U$, existe uma seção $\epsilon^{\prime} \in \boldsymbol{\Gamma}(E)$ tal que $\epsilon=\epsilon^{\prime}$ em alguma vizinhança de $x$.

Demonstração. Seja $s: U \rightarrow \operatorname{FR}(E)$ uma seção local diferenciável de $\operatorname{FR}(E)$, com $x \in U$, e consideremos $\beta_{s}$ a trivialização local de $E$ associada a $s$. Temos $\beta_{s}(x, v)=p$, para um único $v \in \mathbb{R}^{n}$. Seja $f \in \mathrm{C}^{\infty}(M)$ tal que $\operatorname{supp}(f) \subset U$ e $f(x)=1$. Definimos uma seção $\epsilon: U \rightarrow E$ de $E$ por:

$$
\epsilon(y)=\left\{\begin{array}{rll}
\beta_{s}(y, f(y) \cdot v), & \text { se } & y \in U \\
0, & \text { se } & y \notin U
\end{array} .\right.
$$

Temos $\epsilon(x)=p$. Como $\beta_{s}$ é um difeomorfismo diferenciável, $\epsilon$ é diferenciável em $U$. Além disso, como $\operatorname{supp}(f) \subset U, \epsilon$ é nula em uma vizinhança de $M \backslash U$ e, portanto, $\epsilon \in \boldsymbol{\Gamma}(E)$. Para provar o item (b), seja $s: U \rightarrow \mathrm{FR}(E)$ uma seção local diferenciável de $\operatorname{FR}(E)$. Consideremos a representação $\tilde{\epsilon}: U \rightarrow \mathbb{R}^{n}$ de $\epsilon$ em relação a $s$, e $\beta_{s}: U \times \mathbb{R}^{n} \rightarrow \pi^{-1}(U)$ a trivialização local de $E \mathrm{em}$ relação a $s$. Seja $f \in \mathrm{C}^{\infty}(M), \operatorname{com} \operatorname{supp}(f) \subset U$, e tal que $f \equiv 1$ em uma vizinhança $V$ de $x$ em $U$. Definimos uma seção $\epsilon^{\prime}: M \rightarrow E$ por

$$
\epsilon^{\prime}(y)=\left\{\begin{array}{rll}
\beta_{s}(y, f(y) \cdot \tilde{\epsilon}(y)), & \text { se } & y \in U \\
0, & \text { se } & y \notin U
\end{array} .\right.
$$

De (2.17) segue que $\left.\epsilon^{\prime}\right|_{V}=\left.\epsilon\right|_{V}$ e, de forma análoga ao item (a), concluimos que $\epsilon^{\prime} \in \boldsymbol{\Gamma}(E)$.

Definição 2.2.10 Seja $\left(E, \pi, M, \mathbb{R}^{n}\right)$ um fibrado vetorial. Um referencial local em $E$ é uma $n$-upla de seções locais diferenciáveis $\left\{\epsilon_{1}, \ldots, \epsilon_{n}\right\}$ de $E$, definidas em um subconjunto aberto $U$ de $M$, tal que $\left\{\epsilon_{1}(x), \ldots, \epsilon_{n}(x)\right\}$ é uma base para $E_{x}$, para todo $x \in U$.

Quando $U=M$, ou seja, $\epsilon_{i} \in \boldsymbol{\Gamma}(E), 1 \leq i \leq n$, diremos simplesmente que $\left\{\epsilon_{1}, \ldots, \epsilon_{n}\right\}$ é um referencial de $E$. O Lema seguinte estabelece que um referencial local em $E$ é equivalente à existência de uma seção local diferenciável do fibrado principal dos referenciais de $E$. 
Lema 2.2.11 Seja $\left(E, \pi, M, \mathbb{R}^{n}\right)$ um fibrado vetorial. Uma seção local diferenciável $s: U \rightarrow \operatorname{FR}(E)$ de $\operatorname{FR}(E)$ é equivalente à existência de um referencial local $\left\{\epsilon_{1}, \ldots, \epsilon_{n}\right\}$ de $E$.

Demonstração. Fixemos uma base $\left\{v_{1}, \ldots v_{n}\right\}$ de $\mathbb{R}^{n}$. Se $s: U \rightarrow \operatorname{FR}(E)$ é uma seção local diferenciável de $\operatorname{FR}(E)$, definimos $\epsilon_{i}: U \rightarrow E$ por

$$
\epsilon_{i}(x)=\beta_{s}\left(x, v_{i}\right),
$$

para quaisquer $x \in U$ e $1 \leq i \leq n$, onde $\beta_{s}$ é a trivialização local de $E$ correspondente a $s$. Como $\beta_{s}$ é um difeomorfismo diferenciável segue que $\epsilon_{i}$ é de fato uma seção local diferenciável de $E$, para todo $1 \leq i \leq n$. Reciprocamente, dado um referencial local $\left\{\epsilon_{1}, \ldots, \epsilon_{n}\right\}$ de $E$, definimos uma aplicação $\beta: U \times\left.\mathbb{R}^{n} \rightarrow E\right|_{U}$ por

$$
\beta(x, v)=\sum_{i=1}^{n} \alpha_{i} \epsilon_{i}(x),
$$

onde $v=\sum \alpha_{i} v_{i}$. Para cada $x \in U$, a aplicação $\beta_{x}: \mathbb{R}^{n} \rightarrow E_{x}$, dada por $\beta_{x}(v)=\beta(x, v)$, para todo $v \in \mathbb{R}^{n}$, é um isomorfismo linear, logo $\beta$ é bijetora. Mostremos que $\beta$ tem posto máximo. Para isso, seja $\gamma: V \times\left.\mathbb{R}^{n} \rightarrow E\right|_{V}$ uma trivialização local de $E$, com $x \in V \subset U$. Temos

$$
\left(\gamma^{-1} \circ \beta\right)(y, v)=\left(y,\left(\gamma_{y}^{-1} \circ \beta_{y}\right)(v)\right),
$$

para quaisquer $y \in V$ e $v \in \mathbb{R}^{n}$. Agora, como $\gamma_{y}, \beta_{y}: \mathbb{R}^{n} \rightarrow E_{y}$ são isomorfismos lineares, para cada $y \in V$, segue que $\gamma^{-1} \circ \beta: V \times \mathbb{R}^{n} \rightarrow V \times \mathbb{R}^{n}$ é um difeomorfismo diferenciável. Assim, $\beta$ tem posto máximo em $V \times \mathbb{R}^{n}$. Logo, como $x \in U$ é arbitrário, segue que $\beta$ é um difeomorfismo diferenciável. Finalmente, definimos $s: U \rightarrow \operatorname{FR}(E)$ por $s(x) \cdot v=\beta(x, v)$, para quaisquer $x \in U$ e $v \in \mathbb{R}^{n}$. Como $\beta$ é um difeomorfismo segue que $s$ é diferenciável.

Definição 2.2.12 Uma métrica semi-Riemanniana em um fibrado vetorial $\left(E, \pi, M, \mathbb{R}^{n}\right)$ é uma seção diferenciável $g$ do fibrado vetorial $\operatorname{Lin}_{2}(E, \mathbb{R})$ tal que, para todo $x \in M, g_{x}: E_{x} \times E_{x} \rightarrow \mathbb{R}$ é um produto escalar semiRiemanniano em $E_{x}$.

Proposição 2.2.13 Sejam $\left(E, \pi, M, \mathbb{R}^{n}\right)$ um fibrado vetorial, $g$ uma métrica Riemanniana em $E$ e assuma que $\mathbb{R}^{n}$ está munido do produto interno canônico $\langle\cdot, \cdot\rangle$. Então, para todo $y \in M$, existe uma seção local diferenciável $s: U \rightarrow \mathrm{FR}(E)$ de $\mathrm{FR}(E)$ tal que $y \in U$ e $s(x): \mathbb{R}^{n} \rightarrow E_{x}$ é uma isometria linear para todo $x \in U$. 
Demonstração. Seja $\left\{\epsilon_{1}, \ldots, \epsilon_{n}\right\}$ um referencial local de $E$ definido em um aberto $U$ de $M$, com $y \in U$. Pelo processo de ortonormalização de Gram-Schmidt, podemos substituir tais seções por seções locais diferenciáveis $\tilde{\epsilon}_{1}, \ldots, \tilde{\epsilon}_{n}: U \rightarrow E$ tais que $\left\{\tilde{\epsilon}_{1}(x), \ldots, \tilde{\epsilon}_{n}(x)\right\}$ é base ortonormal de $E_{x}$, para todo $x \in U$. Consideremos a seção local diferenciável $s: U \rightarrow \mathrm{FR}(E)$ dada pelo Lema 2.2.11, onde a base $\left\{v_{1}, \ldots, v_{n}\right\}$ de $\mathbb{R}^{n}$, neste caso, é ortonormal. Por definição de $s$, segue que $s(x): \mathbb{R}^{n} \rightarrow E_{x}$ é uma isometria linear, para todo $x \in U$.

Lema 2.2.14 Sejam $E, F$ fibrados vetoriais sobre a mesma variedade diferenciável $M$ e $L: \boldsymbol{\Gamma}(E) \rightarrow \boldsymbol{\Gamma}(F)$ uma aplicação $\mathrm{C}^{\infty}(M)$-linear.

(a) Para quaisquer $\epsilon_{1}, \epsilon_{2} \in \boldsymbol{\Gamma}(E)$ tais que $\left.\epsilon_{1}\right|_{U}=\left.\epsilon_{2}\right|_{U}$, para algum subconjunto aberto $U \subset M$, tem-se $L\left(\epsilon_{1}\right)(x)=L\left(\epsilon_{2}\right)(x)$, para todo $x \in V$, onde $V \subset U$ é um subconjunto aberto.

(b) Para cada $x \in M$, existe uma aplicação linear $L_{x}: E_{x} \rightarrow F_{x}$ tal que $L(\epsilon)(x)=L_{x}(\epsilon(x))$, para toda seção $\epsilon \in \boldsymbol{\Gamma}(E)$.

Demonstração. Seja $f \in \mathrm{C}^{\infty}(M)$ tal que $\operatorname{supp}(f) \subset U$ e $f \equiv 1$ em algum subconjunto aberto $V \subset U$. Temos $f\left(\epsilon_{1}-\epsilon_{2}\right)=0$ e, assim, $f L\left(\epsilon_{1}-\epsilon_{2}\right)=0$. Portanto, $L\left(\epsilon_{1}\right)(x)-L\left(\epsilon_{2}\right)(x)=0$, para todo $x \in V$, provando o item (a). Para provar o item (b), seja $x \in M$. Para cada $v \in E_{x}$, segue do Lema 2.2.9 que existe $\epsilon \in \boldsymbol{\Gamma}(E) \operatorname{com} \epsilon(x)=v$. Defina $L_{x}: E_{x} \rightarrow F_{x}$ por

$$
L_{x}(v)=L(\epsilon)(x)
$$

Do item (a) segue que $L_{x}$ está bem definida e $L(\epsilon)(x)=L_{x}(\epsilon(x))$, para todo $\epsilon \in \boldsymbol{\Gamma}(E)$. Claramente $L_{x}$ é linear.

\subsubsection{Morfismos de fibrados vetoriais}

Sejam $E, F$ fibrados vetoriais sobre a mesma base $M$. Um morfismo de fibrados vetoriais é uma aplicação diferenciável $S: E \rightarrow F$ tal que, para todo $x \in M, S\left(E_{x}\right) \subset F_{x}$ e $S_{x}=\left.S\right|_{E_{x}}: E_{x} \rightarrow F_{x}$ é uma aplicação linear. Se, além disso, $S$ é uma aplicação bijetora, $S: E \rightarrow F$ é chamado um isomorfismo de fibrados vetoriais.

Lema 2.2.15 Seja $S: E \rightarrow F$ um morfismo de fibrados vetoriais. Então, $S$ é um isomorfismo de fibrados vetoriais se, e somente se, $S_{x}: E_{x} \rightarrow F_{x}$ é um isomorfismo linear, para todo $x \in M$. Neste caso, $S$ é um difeomorfismo diferenciável e a aplicação $S^{-1}: F \rightarrow E$ também é um isomorfismo de fibrados vetoriais. 
Demonstração. Se $S$ é um isomorfismo de fibrados vetoriais é claro que $S_{x}: E_{x} \rightarrow F_{x}$ é isomorfismo linear para todo $x \in M$. Reciprocamente, como $S$ é uma aplicação bijetora, resta mostrar que $S^{-1}$ é diferenciável. Como isso é um problema local, podemos assumir que $E=F=M \times \mathbb{R}^{n}$. Neste caso, $S: M \times \mathbb{R}^{n} \rightarrow M \times \mathbb{R}^{n}$ tem a forma

$$
S(x, v)=\left(x, T_{x}(v)\right),
$$

onde $T_{x}: \mathbb{R}^{n} \rightarrow \mathbb{R}^{n}$ é um isomorfismo linear. A matriz Jacobiana de $S$ no ponto $(x, v)$ tem a forma

$$
\left(\begin{array}{cc}
\mathrm{Id} & 0 \\
X & T_{x}
\end{array}\right)
$$

onde $T_{x}$ é a matriz de $T_{x}: \mathbb{R}^{n} \rightarrow \mathbb{R}^{n}$. Como $T_{x}$ é um isomorfismo linear, a matriz acima é invertível, e o Lema segue então do Teorema da Aplicação Inversa.

\subsubsection{Pull-back de fibrados vetoriais}

Sejam $\left(E, \pi, M, \mathbb{R}^{n}\right)$ um fibrado vetorial, $N$ uma variedade diferenciável e $f: N \rightarrow M$ uma aplicação diferenciável. O pull-back de $E$ por $f$ é o subconjunto $f^{*} E$ de $N \times E$ definido por

$$
f^{*} E=\bigcup_{y \in N}\left(\{y\} \times E_{f(y)}\right)
$$

A restrição a $f^{*} E$ da projeção sobre o primeiro fator define uma aplicação $\pi_{1}: f^{*} E \rightarrow N$ e a restrição a $f^{*} E$ da projeção sobre os segundo fator define uma aplicação $\bar{f}: f^{*} E \rightarrow E$, de modo que o diagrama seguinte comuta:

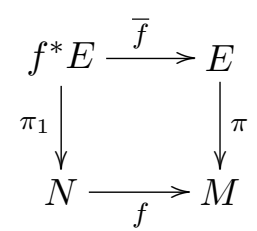

Note que $\left(\pi_{1}, \bar{f}\right): f^{*} E \rightarrow N \times E$ é simplesmente a aplicação inclusão.

Para cada $y \in M$, identifiquemos a fibra $\left(f^{*} E\right)_{y}=\{y\} \times E_{f(y)}$ de $f^{*} E$ com a fibra $E_{f(y)}$ de $E$. Assim, cada fibra de $f^{*} E$ tem a estrutura de espaço vetorial real isomorfo a $\mathbb{R}^{n}$. O conjunto $\operatorname{FR}\left(f^{*} E\right)$ pode ser naturalmente identificado com o pull-back $f^{*} \mathrm{FR}(E)$. Esta identificação torna $\mathrm{FR}\left(f^{*} E\right)$ um $\mathrm{GL}\left(\mathbb{R}^{n}\right)$-fibrado principal e, assim, $\left(f^{*} E, \pi_{1}, N, \mathbb{R}^{n}\right)$ é um fibrado vetorial.

O Lema seguinte permite-nos uma melhor compreensão da estrutura diferenciável do espaço total $f^{*} E$. 
Lema 2.2.16 Sejam $\left(E, \pi, M, \mathbb{R}^{n}\right)$ um fibrado vetorial, $N$ uma variedade diferenciável e $f: N \rightarrow M$ uma aplicação diferenciável. Então, a aplicação $\left(\pi_{1}, \bar{f}\right): f^{*} E \rightarrow N \times E$ é um mergulho diferenciável cuja imagem é o conjunto dos pares $(y, e) \in N \times E$ tais que $f(y)=\pi(e)$.

Demonstração. A comutatividade do diagrama (2.19) implica que a imagem da aplicação $\left(\pi_{1}, \bar{f}\right)$ é o conjunto dos pares $(y, e) \in N \times E$ tais que $f(y)=\pi(e)$. Em virtude da Proposição 1.1.6, a fim de mostrar que $\left(\pi_{1}, \bar{f}\right): f^{*} E \rightarrow N \times E$ é um mergulho diferenciável, é suficiente mostrar que, para toda seção local diferenciável $s: U \rightarrow \mathrm{FR}(E)$ de $\mathrm{FR}(E)$, a restrição da aplicação $\left(\pi_{1}, \bar{f}\right)$ ao conjunto aberto $\left(\pi_{1}, \bar{f}\right)^{-1}\left(f^{-1}(U) \times E\right)=\left.\left(f^{*} E\right)\right|_{f^{-1}(U)}$ é um mergulho diferenciável. Seja $\sigma=s \circ f$ e considere a única seção local $\bar{\sigma}: f^{-1}(U) \rightarrow \operatorname{FR}\left(f^{*} E\right)$ de $\operatorname{FR}\left(f^{*} E\right)$ tal que $\bar{f} \circ \bar{\sigma}=\sigma$. Temos, assim, o seguinte diagrama comutativo:

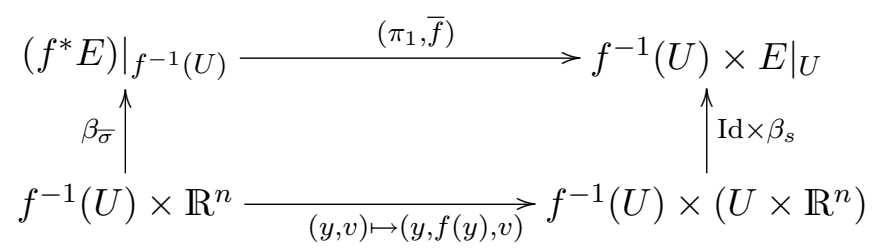

no qual as flechas veticais são difeomorfismos diferenciáveis. Como a flecha horizontal abaixo é um mergulho diferenciável, segue o resultado.

Corolário 2.2.17 Sejam $\left(E, \pi, M, \mathbb{R}^{n}\right)$ um fibrado vetorial, $N$ uma variedade diferenciável e $f: N \rightarrow M$ uma aplicação diferenciável. Sejam também $X$ uma variedade diferenciável e $\phi_{1}: X \rightarrow N, \phi_{2}: X \rightarrow E$ aplicações diferenciáveis tais que $\pi \circ \phi_{2}=f \circ \phi_{1}$. Então, existe uma única aplicação diferenciável $\phi: X \rightarrow f^{*} E$ tal que $\pi_{1} \circ \phi=\phi_{1}$ e $\bar{f} \circ \phi=\phi_{2}$.

Demonstração. A hipótese $\pi \circ \phi_{2}=f \circ \phi_{1}$ significa que a imagem da aplicação $\left(\phi_{1}, \phi_{2}\right): X \rightarrow N \times E$ está contida na imagem da aplicação injetora $\left(\pi_{1}, \bar{f}\right): f^{*} E \rightarrow N \times E$. Assim, existe uma única aplicação $\phi: X \rightarrow f^{*} E$ tal que $\left(\pi_{1}, \bar{f}\right) \circ \phi=\left(\phi_{1}, \phi_{2}\right)$. Como $\left(\pi_{1}, \bar{f}\right)$ é um mergulho e $\left(\phi_{1}, \phi_{2}\right)$ é diferenciável, segue que $\phi$ é diferenciável ${ }^{5}$.

\footnotetext{
${ }^{5}$ Aqui, estamos usando o seguinte resultado de variedades diferenciáveis: Seja $f: M \rightarrow$ $N$ uma imersão diferenciável. Uma aplicação $g: X \rightarrow M$ é diferenciável se, e somente se, $g$ é contínua e $f \circ g: X \rightarrow N$ é diferenciável (cf. Observação 1.1.4).
} 
Exemplo 2.2.18 Sejam $M, \bar{M}$ variedades diferenciáveis e $f: M \rightarrow \bar{M}$ uma aplicação diferenciável. Denote por $\pi: T M \rightarrow M$ e $\bar{\pi}: T \bar{M} \rightarrow \bar{M}$ as projeções canônicas. Aplicando o Corolário 2.2.17 $\operatorname{com} X=T M, \phi_{1}=\pi$, $\phi_{2}=d f: T M \rightarrow T \bar{M}$ e $E=T \bar{M}$, obtemos uma aplicação (que também denotaremos por $\mathrm{d} f) \mathrm{d} f: T M \rightarrow f^{*} T \bar{M}$ tal que $\bar{f} \circ \mathrm{d} f=\mathrm{d} f$ e $\pi_{1} \circ \mathrm{d} f=\pi$. Além disso, $\mathrm{d} f: T M \rightarrow f^{*} T \bar{M}$ é um morfismo de fibrados vetoriais. O diagrama seguinte ilustra a situação.

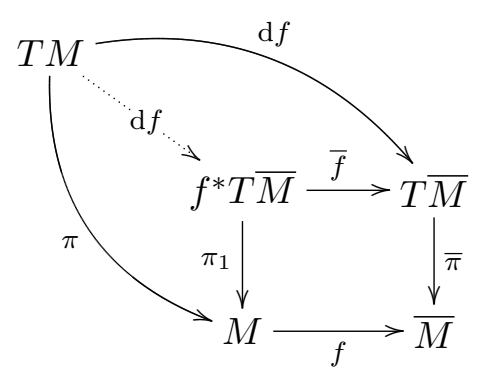

\subsubsection{Subfibrados vetoriais}

Sejam $\left(E, \pi, M, \mathbb{R}^{n}\right)$ um fibrado vetorial, $V$ um subespaço vetorial de $\mathbb{R}^{n}$ e $F$ um subconjunto de $E$ tais que, para todo $x \in M$, o conjunto $F_{x}=$ $F \cap E_{x}$ é um subespaço vetorial da fibra $E_{x}$ com a mesma dimensão de $V$. Dado $x \in M$, dizemos que um referencial $p \in \mathrm{FR}\left(E_{x}\right)$ é adaptado a $(V, F)$ se $p(V)=F_{x}$. Denotaremos por $\mathrm{FR}\left(E_{x} ; V, F_{x}\right)$ o conjunto de todos os referenciais de $E_{x}$ que são adaptados a $(V, F)$.

Denotemos por $\mathrm{GL}\left(\mathbb{R}^{n} ; V\right)$ o subgrupo de Lie de $\mathrm{GL}\left(\mathbb{R}^{n}\right)$ formado por todos os isomorfismos lineares $g: \mathbb{R}^{n} \rightarrow \mathbb{R}^{n}$ tais que $g(V F)=V$. Assim, o conjunto $\operatorname{FR}\left(E_{x} ; V, F_{x}\right)$ é uma $\mathrm{GL}\left(\mathbb{R}^{n} ; V\right)$-órbita, para todo $x \in M$. Definimos o conjunto:

$$
\operatorname{FR}(E ; V, F)=\bigcup_{x \in M} \operatorname{FR}\left(E_{x} ; V, F_{x}\right)
$$

de todos os referenciais de $E$ adaptados a $(V, F)$.

Definição 2.2.19 Seja $\left(E, \pi, M, \mathbb{R}^{n}\right)$ um fibrado vetorial. Um subconjunto $F \subset E$ é chamado um subfibrado vetorial se existe um subespaço vetorial $V$ de $\mathbb{R}^{n}$ tal que, para todo $x \in M$, o conjunto $F_{x}=F \cap E_{x}$ é um subespaço vetorial de $E_{x}$ com mesma dimensão de $V$ e $\mathrm{FR}(E ; V, F)$ é um $\mathrm{GL}\left(\mathbb{R}^{n} ; V\right)$ subfibrado principal de $\operatorname{FR}(E)$. 
A segunda condição da Definição 2.2.19 significa que todo ponto de $M$ pertence ao domínio $U$ de uma seção local diferenciável $s: U \rightarrow \operatorname{FR}(E)$ de $\operatorname{FR}(E)$ tal que $s(x)(V)=F_{x}$, para todo $x \in U$.

Mostraremos agora que todo subfibrado vetorial $(F, \pi, M, V)$ de um fibrado vetorial $\left(E, \pi, M, \mathbb{R}^{n}\right)$ também é um fibrado vetorial. De fato, a projeção $\pi: E \rightarrow M$ restringe-se a uma aplicação $\left.\pi\right|_{F}: F \rightarrow M$ e, para todo $x \in M$, a fibra $F_{x}$ possui a estrutura de espaço vetorial real isomoformo a $V$. Para que $F$ seja um fibrado vetorial sobre $M$ com fibra típica $V$, devemos exibir um atlas maximal de seções locais de $\Pi: \operatorname{FR}(F) \rightarrow M$.

Dado $p \in \operatorname{FR}(E ; V, F)$, temos que $\left.p\right|_{V}$ é um referencial de $F$. Assim, obtemos uma aplicação

$$
\phi: \operatorname{FR}(E ; V, F) \rightarrow \mathrm{FR}(F),
$$

definida por $\phi(p)=\left.p\right|_{V}$ para todo $p \in \mathrm{FR}(E ; V, F)$. A aplicação $\phi$ preserva fibra, isto é, $\phi\left(\operatorname{FR}\left(E_{x} ; V, F_{x}\right)\right) \subset \mathrm{FR}\left(F_{x}\right)$ para todo $x \in M$ e, restrita a cada fibra $\operatorname{FR}\left(E_{x} ; V, F_{x}\right)$, satisfaz:

$$
\phi(p \circ g)=\phi(p) \circ T(g),
$$

para quaisquer $p \in \mathrm{FR}\left(E_{x} ; V, F_{x}\right)$ e $g \in \mathrm{GL}\left(\mathbb{R}^{n} ; V\right)$, onde

$$
T: \operatorname{GL}\left(\mathbb{R}^{n} ; V\right) \rightarrow \mathrm{GL}(V)
$$

é o homomorfismo de grupos de Lie dado por $T(g)=\left.g\right|_{V}$ para todo $g \in$ $\mathrm{GL}\left(\mathbb{R}^{n} ; V\right)$. Consideremos o seguinte conjunto de seções locais de $\Pi: \operatorname{FR}(F) \rightarrow$ $M$ :

$$
\mathcal{A}=\{\phi \circ s: s \text { é seção local diferenciável de } \operatorname{FR}(E ; V, F)\} .
$$

Mostremos que $\mathcal{A}$ é um atlas de seções locais de $\Pi: \operatorname{FR}(F) \rightarrow M$. De fato, os domínios de seções locais de $\mathcal{A}$ formam uma cobertura para $M$. Além disso, se $s_{1}: U_{1} \rightarrow \mathrm{FR}(E ; V, F)$ e $s_{2}: U_{2} \rightarrow \mathrm{FR}(E ; V, F)$ são seções locais diferenciáveis de $\operatorname{FR}(E ; V, F)$, com aplicação de transição $g: U_{1} \cap U_{2} \rightarrow$ $\operatorname{GL}\left(\mathbb{R}^{n} ; V\right)$, então a aplicação de transição de $\phi \circ s_{1}$ para $\phi \circ s_{2}$ é a aplicação

$$
T \circ g: U_{1} \cap U_{2} \rightarrow \mathrm{GL}(V) .
$$

Assim, $\phi \circ s_{1}$ e $\phi \circ s_{2}$ são compatíveis e (2.21) é, de fato, um atlas de seções locais de $\Pi: \operatorname{FR}(F) \rightarrow M$. Considerando então $\mathcal{A}_{\max }$ o único atlas maximal de seções locais de $\operatorname{FR}(F)$ contendo (2.21), concluimos que $\operatorname{FR}(F)$ é um $\mathrm{GL}(V)$-fibrado principal tal que aplicação $\phi$ dada em (2.20) é um morfismo de fibrados principais. Portanto, $\left(F,\left.\pi\right|_{F}, M, V\right)$ é um fibrado vetorial. 
Proposição 2.2.20 Um subfibrado vetorial $F$ de um fibrado vetorial $E$ é uma subvariedade regular de $E$ e a estrutura diferenciável de $F$, como espaço total de um fibrado vetorial, coincide com a estrutura diferenciável induzida de $E$ como subvariedade regular.

Demonstração. Para provar que $F$ é subvariedade regular de $E$, basta mostrar que a aplicação de inclusão i : $F \rightarrow E$ é um mergulho diferenciável. Em virtude da Proposição 1.1.6, a fim de que $\mathfrak{i}: F \rightarrow E$ seja um mergulho diferenciável, basta mostrar que para toda seção local diferenciável $s: U \rightarrow$ $\mathrm{FR}(E ; V, F)$ de $\mathrm{FR}(E ; V, F)$, a restrição da aplicação de inclusão $\mathfrak{i}: F \rightarrow$ $E$ ao conjunto aberto $\mathfrak{i}^{-1}\left(\pi^{-1}(U)\right)=\left(\left.\pi\right|_{F}\right)^{-1}(U)=\left.F\right|_{U}$ é um mergulho diferenciável. Como $\mathrm{FR}(E ; V, F)$ é um $\mathrm{GL}\left(\mathbb{R}^{n} ; V\right)$-subfibrado principal de $\operatorname{FR}(E)$, temos que $\mathfrak{i} \circ s$ é uma seção local diferenciável de $\mathrm{FR}(E)$, onde $\mathfrak{i}: \operatorname{FR}(E ; V, F) \rightarrow \operatorname{FR}(E)$ é a aplicação de inclusão. Além disso, $\phi \circ s$ é uma seção local diferenciável de $\mathrm{FR}(F)$, onde $\phi$ é o morfismo definido em (2.20). Temos, assim, o seguinte diagrama comutativo:

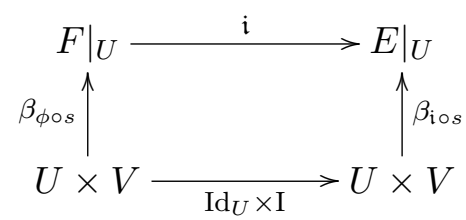

onde I : $V \rightarrow \mathbb{R}^{n}$ é a aplicação de inclusão. Como as flechas verticais são difeomorfismos diferenciáveis e a flecha horizontal abaixo é um mergulho diferenciável, segue que a aplicação de inclusão $i: F \rightarrow E$ é um mergulho diferenciável.

\subsection{Conexões em fibrados principais}

Seja $P(M, \Pi, G)$ um fibrado principal. Uma distribuição $\operatorname{Hor}(P)$ na variedade diferenciável $P$ é chamada horizontal em relação a $\Pi$ se

$$
T_{p} P=\operatorname{Hor}_{p}(P) \oplus \operatorname{Ver}_{p}(P),
$$

para todo $p \in P$.

Definição 2.3.1 Uma conexão em $P$ é uma distribuição horizontal diferenciável $\operatorname{Hor}(P)$ em $P$ que é $G$-invariante, ou seja,

$$
\mathrm{d} \gamma_{g}\left(\operatorname{Hor}_{p}(P)\right)=\operatorname{Hor}_{p \cdot g}(P),
$$

para quaisquer $p \in P$ e $g \in G$, onde $\gamma_{g}: P \rightarrow P$ é o difeomorfismo dado pela ação de $g$ em $P$. 
O conjunto

$$
\operatorname{Ver}(P)=\bigcup_{p \in P} \operatorname{Ver}_{p}(P) \subset T P
$$

é uma distribuição diferenciável em $P$, chamada a distribuição vertical em $P$. De (2.6) segue que $\operatorname{Ver}(P)$ também é uma distribuição $G$-invariante.

Seja $\operatorname{Hor}(P)$ uma conexão em $P$. Associado à conexão $\operatorname{Hor}(P)$, o isomorfismo canônico (2.5) define uma 1-forma $\omega$ em $P$ à valores em $\mathfrak{g}$ tal que $\operatorname{ker}\left(\omega_{p}\right)=\operatorname{Hor}_{p}(P)$, para todo $p \in P$. Mais precisamente, definimos:

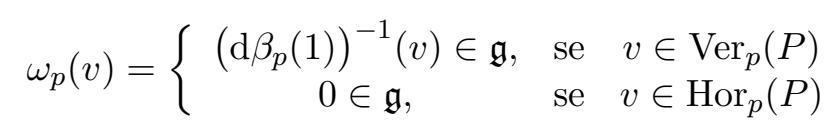

para quaisquer $p \in P$ e $v \in T_{p} P$, onde $\left(\mathrm{d} \beta_{p}(1)\right)^{-1}: \operatorname{Ver}_{p}(P) \rightarrow \mathfrak{g}$ é o inverso do isomorfismo (2.5).

Lema 2.3.2 A 1-forma $\omega$, definida em (2.23), é diferenciável.

Demonstração. Consideremos a aplicação $L_{\omega}: T P \rightarrow P \times \mathfrak{g}$, cuja restrição a $T_{p} P$ é dada por

$$
L_{\omega}(v)=\left(p, \omega_{p}(v)\right)
$$

para quaisquer $p \in P$ e $v \in T_{p} P$. Mostremos que $L_{\omega}$ é um morfismo de fibrados vetoriais. Para isso, consideremos a aplicação $L_{\beta}: P \times \mathfrak{g} \rightarrow \operatorname{Ver}(P)$ definida por

$$
L_{\beta}(p, X)=\mathrm{d} \beta_{p}(1) \cdot X,
$$

para quaisquer $p \in P$ e $X \in \mathfrak{g}$. A aplicação $L_{\beta}$ é diferenciável, pois é a restrição a $P \times \mathfrak{g} \subset T P \times T G$ da diferencial da ação diferenciável à direita $P \times G \rightarrow P$. Assim, $L_{\beta}$ é um isomorfismo de fibrados vetoriais. Como $\operatorname{Hor}(P)$ é uma distribuição diferenciável, a projeção $\pi_{\text {ver }}: T P \rightarrow \operatorname{Ver}(P)$, cuja restrição a $T_{p} P$ é a projeção sobre $\operatorname{Ver}_{p}(P)$, é um morfismo de fibrados vetoriais. Portanto, $L_{\omega}=L_{\beta}^{-1} \circ \pi_{\mathrm{ver}}: T P \rightarrow P \times \mathfrak{g}$ é um morfismo de fibrados vetoriais, o que implica que $\omega$ é diferenciável.

Seja $\operatorname{Hor}(P)$ uma conexão em $P$. Considerando a 1-forma diferenciável $\omega$, definida em (2.23), temos o seguinte diagrama comutativo:

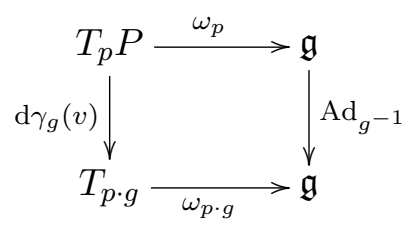


Basta verificar que $\operatorname{Ad}_{g^{-1}} \circ \omega_{p}$ e $\omega_{p \cdot g} \circ \mathrm{d} \gamma_{g}(p)$ coincidem em $\operatorname{Hor}_{p}(P)$ e $\operatorname{Ver}_{p}(P)$, usando a comutatividade do diagrama (2.7). A comutatividade do diagrama (2.26) é equivalente a

$$
\gamma_{g}^{*} \omega=\operatorname{Ad}_{g^{-1}} \circ \omega
$$

para todo $g \in G$.

Definição 2.3.3 Uma 1-forma $\omega$ em $P$ à valores em $\mathfrak{g}$ que satisfaz a relação (2.27) é chamada Ad-pseudo G-invariante.

Definição 2.3.4 Seja $P(M, \Pi, G)$ um fibrado principal. Uma 1-forma diferenciável Ad-pseudo $G$-invariante $\omega$ definida em $P$ à valores em $\mathfrak{g}$ que satisfaz a condição:

$$
\left.\omega_{p}\right|_{\operatorname{Ver}_{p}(P)}=\left(\mathrm{d} \beta_{p}(1)\right)^{-1},
$$

para todo $p \in P$, é chamada uma forma de conexão em $P$.

Toda forma de conexão $\omega$ em $P$ define uma conexão $\operatorname{Hor}(P)$ em $P$. Mais precisamente, temos a seguinte:

Proposição 2.3.5 Existe uma correspondência biunívoca entre conexões $\operatorname{Hor}(P)$ em $P$ e formas de conexão $\omega$ em $P$.

Demonstração. Se Hor $(P)$ é uma conexão em $P$ então a forma de conexão $\omega$ em $P$ é a 1-forma $\omega$ definida em (2.23). Reciprovamente, seja $\omega$ uma forma de conexão em $P$. Definimos a distribuição $\operatorname{Hor}(P)$ por

$$
\operatorname{Hor}_{p}(P)=\operatorname{ker}\left(\omega_{p}\right)
$$

para todo $p \in P$. Como $\left.\omega_{p}\right|_{\operatorname{Ver}_{p}(P)}$ é um isomorfismo linear segue que $\operatorname{Hor}(P)$ é horizontal. Mostremos que Hor $(P)$ é diferenciável. Como $\omega$ é diferenciável, a aplicação $L_{\omega}$, definida em (2.24), é diferenciável e, portanto, é um morfismo de fibrados vetoriais. Como $L_{\omega}$ é sobrejetora, seu núcleo $\operatorname{ker}\left(L_{\omega}\right)=\operatorname{Hor}(P)$ é um subfibrado vetorial de $T P$. Assim, $\operatorname{Hor}(P)$ é uma distribuição diferenciável em $P$. Finalmente, mostremos que $\operatorname{Hor}(P)$ é $G$-invariante. Como $\omega$ satisfaz (2.27), o diagrama (2.26) comuta, para quaisquer $p \in P$ e $g \in G$. Assim,

$$
\mathrm{d} \gamma_{g}\left(\operatorname{Hor}_{p}(P)\right) \subset \operatorname{Hor}_{p \cdot g}(P),
$$

para quaisquer $p \in P$ e $g \in G$. Trocando $p$ por $p \cdot g$ e $g$ por $g^{-1}$ em (2.30), obtemos a inclusão contrária $\operatorname{Hor}_{p \cdot g}(P) \subset \mathrm{d} \gamma_{g}\left(\operatorname{Hor}_{p}(P)\right)$. Portanto, $\operatorname{Hor}(P)$ é $G$-invariante. 
Exemplo 2.3.6 Sejam $P(M, \Pi, G)$ um fibrado principal e $\operatorname{Hor}(P)$ uma conexão em $P$. Se $U$ é um subconjunto aberto de $M$, então a interseção $\operatorname{Hor}(P) \cap T\left(\left.P\right|_{U}\right)$ é uma conexão em $\left.P\right|_{U}$. A forma de conexão associada a $\operatorname{Hor}(P) \cap T\left(\left.P\right|_{U}\right)$ é a restrição da forma de conexão associada a Hor $(P)$.

Lema 2.3.7 Sejam $P(M, \Pi, G)$ um fibrado principal e $s: U \rightarrow P$ uma seção local diferenciável de $P$. Se $\bar{\omega}$ é uma 1-forma em $U$ à valores em $\mathfrak{g}$ então existe uma única 1-forma $\omega$ em $\left.P\right|_{U}$ à valores em $\mathfrak{g}$ Ad-pseudo $G$-invariante satisfazendo (2.28) $\operatorname{com} s^{*} \omega=\bar{\omega}$. Além disso, $\omega$ é diferenciável se, e somente se, $\bar{\omega}$ é diferenciável.

Demonstração. Mostremos inicialmente que o Lema é válido no caso em que $P=M \times G$ é o $G$-fibrado principal trivial. Seja $\omega$ uma 1-forma em $P$ à valores em $\mathfrak{g}$. Diferenciando a ação à direita de $G$ em $P$, obtemos uma ação à direita de $G$ em $T P$ dada por:

$$
(v, X) \cdot g=\mathrm{d} \gamma_{g}(v, X)=\left(v, \mathrm{~d} R_{g}(X)\right),
$$

para quaisquer $v \in T M, X \in T G$ e $g \in G$. Observe que $\omega$ é Ad-pseudo $G$-invariante se, e somente se, a igualdade (2.27) é válida nos pontos de $M \times\{1\} \subset P$, i.e., se, e somente se:

$$
\omega_{(x, g)}\left(v, \mathrm{~d} R_{g}(X)\right)=\operatorname{Ad}_{g^{-1}}\left(\omega_{(x, 1)}(v, X)\right),
$$

para quaisquer $x \in M, v \in T_{x} M, g \in G$ e $X \in \mathfrak{g}$. Seja $\bar{\omega}$ a 1-forma em $M$ à valores em $\mathfrak{g}$ que é o pull-back de $\omega$ pela seção diferenciável $s_{1}: M \rightarrow P$, dada por $s_{1}(x)=(x, 1)$. A igualdade $s_{1}^{*} \omega=\bar{\omega}$ significa que:

$$
\omega_{(x, 1)}(v, 0)=\bar{\omega}_{x}(v),
$$

para quaisquer $x \in M$ e $v \in T_{x} M$. Supondo $\omega$ Ad-pseudo $G$-invariante, a condição (2.28) é válida para todo $p \in P$ se, e somente se, vale para todo $p \in M \times\{1\} \subset P$, i.e., se, e somente se:

$$
\omega_{(x, 1)}(0, X)=X
$$

para quaisquer $x \in M$ e $X \in \mathfrak{g}$. As condições (2.31), (2.32) e (2.33) são equivalentes a

$$
\omega_{(x, g)}\left(v, \mathrm{~d} R_{g}(X)\right)=\operatorname{Ad}_{g^{-1}}\left(\bar{\omega}_{x}(v)+X\right),
$$

para quaisquer $x \in M, v \in T_{x} M, g \in G$ e $X \in \mathfrak{g}$. A igualdade (2.34) mostra que, dado uma 1-forma $\bar{\omega}$ em $M$ à valores em $\mathfrak{g}$, existe uma única 1-forma $\omega$ 
em $P=M \times G$ à valores em $\mathfrak{g}$ que é Ad-pseudo $G$-invariante satisfazendo a condição (2.28) para todo $p \in P$, com $s_{1}^{*} \omega=\bar{\omega}$. Note que a igualdade (2.34) implica que $\omega$ é diferenciável se, e somente se, $\bar{\omega}$ é diferenciável. Para provar o caso geral, consideremos o seguinte diagrama comutativo (cf. (2.3)):

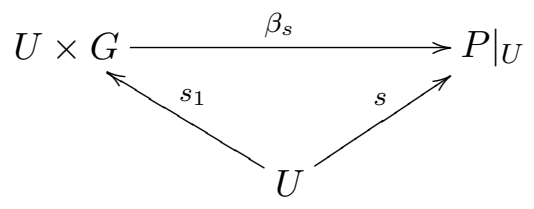

A aplicação $\beta_{s}$ é um isomorfismo de fibrados principais, cujo homomorfismo subjacente de grupos de Lie é a aplicação identidade. Dado uma 1-forma $\omega$ em $\left.P\right|_{U}$ à valores em $\mathfrak{g}$, segue que $\omega$ é Ad-pseudo $G$-invariante e satisfaz (2.28) para todo $\left.p \in P\right|_{U}$ se, e somente se, $\beta_{s}^{*} \omega$ é Ad-pseudo $G$-invariante e satisfaz (2.28) para todo $p \in U \times G$. Além disso, $s^{*} \omega=\bar{\omega}$ se, e somente se, $s_{1}^{*}\left(\beta_{s}^{*} \omega\right)=\bar{\omega}$. Isso prova o caso geral.

Se $\omega$ é a forma de conexão em $P$, correspondente a uma conexão $\operatorname{Hor}(P)$ em $P$, e $s: U \rightarrow P$ é uma seção local diferenciável então a 1-forma $\bar{\omega}=s^{*} \omega$ em $U$ à valores em $\mathfrak{g}$ é chamada a representação de $\omega$ em relação a $s$. O Lema 2.3.7 implica que a forma de conexão $\omega$ em $\left.P\right|_{U}$ é unicamente determinada por sua representação $\bar{\omega}$ em relação a uma seção local diferenciável $s: U \rightarrow P$ fixada.

Sejam $P(M, \Pi, G)$ um fibrado principal, $N$ uma variedade diferenciável e $f: N \rightarrow M$ uma aplicação diferenciável. Toda conexão $\operatorname{Hor}(P)$ em $P$ induz uma conexão no fibrado $f^{*} P$. Mais precisamente, temos o seguite:

Lema 2.3.8 Se $\omega$ é uma forma de conexão em $P$ então $\bar{f}^{*} \omega$ é uma forma de conexão em $f^{*} P$, onde $\bar{f}: f^{*} P \rightarrow P$ é a aplicação canônica do pull-back $f^{*} P$.

Demonstração. Para todo $y \in N$, a restrição de $\bar{f}$ à fibra $\left(f^{*} P\right)_{y}=P_{f(y)}$ é simplesmente a aplicação identidade de $P_{f(y)}$. Assim, desse fato e de (2.28), temos:

$$
\left.\left(\bar{f}^{*} \omega\right)_{p}\right|_{\operatorname{Ver}_{p}\left(f^{*} P\right)}=\left(\mathrm{d} \beta_{p}(1)\right)^{-1},
$$

para todo $p \in f^{*} P$. Para cada $g \in G$, denotemos por $\gamma_{g}^{P}: P \rightarrow P$ a ação de $g$ em $P$ e por $\gamma_{g}^{f^{*} P}: f^{*} P \rightarrow f^{*} P$ a ação de $g$ em $f^{*} P$. Devemos mostrar que

$$
\left(\gamma_{g}^{f^{*} P}\right)^{*}\left(\bar{f}^{*} \omega\right)=\operatorname{Ad}_{g^{-1}} \circ\left(\bar{f}^{*} \omega\right)
$$


De fato, para cada $g \in G$, temos $\gamma_{g}^{P} \circ \bar{f}=\bar{f} \circ \gamma_{g}^{f^{*} P}$. Assim,

$$
\begin{aligned}
\left(\gamma_{g}^{f^{*} P}\right)^{*}\left(\bar{f}^{*} \omega\right) & \left.=\left(\bar{f} \circ \gamma_{g}^{f^{*} P}\right)\right)^{*} \omega=\left(\gamma_{g}^{P} \circ \bar{f}\right)^{*} \omega=\bar{f}^{*}\left(\left(\gamma_{g}^{P}\right)^{*} \omega\right) \\
& =\bar{f}^{*}\left(\operatorname{Ad}_{g^{-1}} \circ \omega\right)=\operatorname{Ad}_{g^{-1}} \circ\left(\bar{f}^{*} \omega\right),
\end{aligned}
$$

provando o Lema.

A correspondência biunívoca entre conexões e formas de conexão em um fibrado principal, dada pela Proposição 2.3.5, motiva a seguinte:

Definição 2.3.9 Sejam $P(M, \Pi, G)$ um fibrado principal, $N$ uma variedade diferenciável e $f: N \rightarrow M$ uma aplicação diferenciável. Dado uma conexão $\operatorname{Hor}(P)$ em $P$, o pull-back de $\operatorname{Hor}(P)$ por $f$ é a conexão $\operatorname{Hor}\left(f^{*} P\right)$ em $f^{*} P$ correspondente à forma de conexão $\bar{f}^{*} \omega$, onde $\omega$ é a forma de conexão em $P$ correspondente à conexão $\operatorname{Hor}(P)$.

\subsection{Conexões em fibrados vetoriais}

Seja $\left(E, \pi, M, \mathbb{R}^{n}\right)$ um fibrado vetorial. Dados $X \in \boldsymbol{\Gamma}(T M)$ e $f \in$ $\mathrm{C}^{\infty}(M)$, denotemos por $X(f)$ a aplicação definida por

$$
X(f)(x)=\mathrm{d} f_{x}(X(x)),
$$

para todo $x \in M$.

Definição 2.4.1 Uma conexão em $E$ é uma aplicação $\mathbb{R}$-bilinear

$$
\nabla: \boldsymbol{\Gamma}(T M) \times \boldsymbol{\Gamma}(E) \rightarrow \boldsymbol{\Gamma}(E),
$$

denotada por $(X, \epsilon) \longmapsto \nabla_{X} \epsilon$, que é $\mathrm{C}^{\infty}(M)$-linear na variável $X$ e que satisfaz a regra de Leibnitz:

$$
\nabla_{X}(f \epsilon)=X(f) \epsilon+f \nabla_{X} \epsilon
$$

para quaisquer $X \in \boldsymbol{\Gamma}(T M), f \in \mathrm{C}^{\infty}(M)$ e $\epsilon \in \boldsymbol{\Gamma}(E)$.

Definição 2.4.2 Uma conexão em uma variedade diferenciável $M$ é uma conexão em seu fibrado tangente $T M$. Uma variedade afim é um par $(M, \nabla)$, onde $M$ é uma variedade diferenciável e $\nabla$ é uma conexão em $M$. 
Observação 2.4.3 Para toda seção $\epsilon \in \boldsymbol{\Gamma}(E)$ e todo ponto $x \in M$, a seção $\nabla_{X} \epsilon$ depende somente em $X(x)$. De fato, como a aplicação

$$
X \in \boldsymbol{\Gamma}(T M) \longmapsto \nabla_{X} \epsilon \in \boldsymbol{\Gamma}(E)
$$

é $\mathrm{C}^{\infty}(M)$-linear, segue do Lema 2.2.14 que, para cada $x \in M$, existe uma aplicação linear

$$
v \in T_{x} M \longmapsto \nabla_{v} \epsilon \in E_{x}
$$

tal que

$$
\nabla_{v} \epsilon=\nabla_{X} \epsilon(x)
$$

para todo $X \in \boldsymbol{\Gamma}(T M)$, com $X(x)=v$. De (2.35) segue que

$$
\nabla_{v}(f \epsilon)=\mathrm{d} f_{x}(v) \cdot \epsilon(x)+f(x) \nabla_{v} \epsilon,
$$

para quaisquer $\epsilon \in \boldsymbol{\Gamma}(E), f \in \mathrm{C}^{\infty}(M), x \in M$ e $v \in T_{x} M$.

O Lema seguinte faz uso da regra de Leibnitz para mostrar que $\nabla_{X} \epsilon(x)$ depende somente na restrição de $\epsilon$ em uma vizinhança de $x$.

Lema 2.4.4 Seja $\nabla$ uma conexão em $E$ e sejam dados um ponto $x \in M$ e seções $\epsilon_{1}, \epsilon_{2} \in \boldsymbol{\Gamma}(E)$ tais que $\epsilon_{1}=\epsilon_{2}$ em uma vizinhança $U$ de $x$. Então:

$$
\nabla_{X} \epsilon_{1}(x)=\nabla_{X} \epsilon_{2}(x)
$$

para todo $X \in \boldsymbol{\Gamma}(T M)$.

Demonstração. Seja $f \in \mathrm{C}^{\infty}(M)$ tal que $\operatorname{supp}(f) \subset U$ e $f \equiv 1$ em uma vizinhança $V \subset U$ de $x$. Temos $f \cdot \epsilon_{1}=f \cdot \epsilon_{2} \mathrm{em} M$, assim

$$
\nabla_{X}\left(f \epsilon_{1}\right)=\nabla_{X}\left(f \epsilon_{2}\right) .
$$

Por outro lado, de (2.35) e a escolha de $f$, temos:

$$
\begin{aligned}
\nabla_{X}\left(f \epsilon_{i}\right)(x) & =\mathrm{d} f_{x}(X(x)) \cdot \epsilon_{i}(x)+f(x) \nabla_{X} \epsilon_{i}(x) \\
& =0 \cdot \epsilon_{i}(x)+1 \cdot \nabla_{X} \epsilon_{i}(x)=\nabla_{X} \epsilon_{i}(x),
\end{aligned}
$$

para $i=1,2$, provando a afirmação.

Definição 2.4.5 Sejam $E, E^{\prime}$ fibrados vetoriais sobre a mesma variedade diferenciável $M$ munidos de conexões $\nabla$ e $\nabla^{\prime}$, respectivamente. Dizemos que um morfismo de fibrados vetoriais $L: E \rightarrow E^{\prime}$ preserva conexão se

$$
\nabla_{v}^{\prime}(L \circ \epsilon)=L\left(\nabla_{v} \epsilon\right)
$$

para quaisquer $v \in T M$ e $\epsilon \in \boldsymbol{\Gamma}(E)$. 
Definição 2.4.6 Sejam $M, M^{\prime}$ variedades afins. Uma aplicação diferenciável $f: M \rightarrow M^{\prime}$ é chamada uma aplicação afim se o morfismo de fibrados vetoriais $\mathrm{d} f: T M \rightarrow f^{*} T M^{\prime}$, dado no Exemplo 2.2.18, preserva conexão, i.e.,

$$
\left(f^{*} \nabla^{\prime}\right)_{v}(\mathrm{~d} f \circ X)=\mathrm{d} f\left(\nabla_{v} X\right),
$$

para quaisquer $v \in T M$ e $X \in \boldsymbol{\Gamma}(T M)$.

Uma conexão $\nabla$ em $E$ induz uma conexão em $\left.E\right|_{U}$, para qualquer subconjunto aberto $U$ de $M$. Mais precisamente, temos o seguinte:

Lema 2.4.7 Seja $\nabla$ uma conexão em $E$. Dado um subconjunto aberto $U \subset M$, existe uma única conexão $\nabla^{U}$ no fibrado vetorial $\left.E\right|_{U}$ tal que:

$$
\left.\nabla_{X}^{U} \epsilon\right|_{U}(x)=\nabla_{X} \epsilon(x)
$$

para quaisquer $x \in U, \epsilon \in \boldsymbol{\Gamma}(E)$ e $X \in \boldsymbol{\Gamma}\left(\left.T M\right|_{U}\right)$.

Demonstração. Sejam $\epsilon^{\prime} \in \boldsymbol{\Gamma}\left(\left.E\right|_{U}\right)$ e $x \in U$. Pelo Lema 2.2.9, existe $\epsilon \in \boldsymbol{\Gamma}(E)$ tal que $\epsilon^{\prime}=\epsilon$ em uma vizinhança de $x$ em $U$. Assim, se $\nabla^{U}$ é uma conexão em $\left.E\right|_{U}$ satisfazendo (2.36), o Lema 2.4.4 implica que

$$
\nabla_{X}^{U} \epsilon^{\prime}(x)=\nabla_{X} \epsilon(x)
$$

para todo $X \in \boldsymbol{\Gamma}\left(\left.T M\right|_{U}\right)$, o que prova a unicidade de $\nabla^{U}$. O Lema 2.4.4 implica também que o lado direito de (2.37) não depende da escolha de $\epsilon \in \boldsymbol{\Gamma}(E)$, que é igual a $\epsilon^{\prime}$ em uma vizinhança de $x$ em $U$. Definimos então $\nabla^{U}$ como em (2.37). Claramente $\nabla^{U}$ é uma conexão em $\left.E\right|_{U}$.

A conexão $\nabla^{U}$, definida pelo Lema 2.4.7, é chamada a conexão induzida em $\left.E\right|_{U}$. Convencionaremos em denotar a conexão $\nabla^{U}$ pelo mesmo símbolo $\nabla$ usado para denotar a conexão de $E$.

Exemplo 2.4.8 Sejam $\left(E, \pi, M, \mathbb{R}^{n}\right)$ um fibrado vetorial e $s: U \rightarrow \mathrm{FR}(E)$ uma seção local diferenciável. Definimos uma conexão $\mathrm{dI}^{s}$ associada a $s$ no fibrado vetorial $\left.E\right|_{U}$ por:

$$
\mathrm{dI}_{v}^{s} \epsilon(x)=s(x) \cdot \mathrm{d} \tilde{\epsilon}_{x}(v),
$$

para quaisquer $x \in U, v \in \boldsymbol{\Gamma}\left(\left.T M\right|_{U}\right)$ e $\epsilon \in \boldsymbol{\Gamma}\left(\left.E\right|_{U}\right)$, onde $\tilde{\epsilon}: U \rightarrow \mathbb{R}^{n}$ é a representação de $\epsilon$ em relação a $s$. 
Definição 2.4.9 Sejam $\nabla$ uma conexão em $E$ e $s: U \rightarrow \mathrm{FR}(E)$ uma seção local diferenciável. O tensor de Christoffel da conexão $\nabla$ relativo a $s$ é a aplicação $\mathrm{C}^{\infty}(M)$-bilinear

$$
\Gamma: \boldsymbol{\Gamma}\left(\left.T M\right|_{U}\right) \times \boldsymbol{\Gamma}\left(\left.E\right|_{U}\right) \rightarrow \boldsymbol{\Gamma}\left(\left.E\right|_{U}\right)
$$

definida por

$$
\Gamma=\nabla-\mathrm{dI}^{s},
$$

onde $\mathrm{dI}^{s}$ é a conexão definida em (2.38).

Como $\Gamma$ é uma aplicação $\mathrm{C}^{\infty}(M)$-bilinear, segue do Lema 2.2.14 que, para cada $x \in U$, existe uma aplicação bilinear

$$
\Gamma_{x}: T_{x} M \times E_{x} \rightarrow E_{x}
$$

tal que

$$
\Gamma_{x}(X(x), \epsilon(x))=\Gamma(X, \epsilon)(x),
$$

para quaisquer $X \in \boldsymbol{\Gamma}\left(\left.T M\right|_{U}\right)$ e $\epsilon \in \boldsymbol{\Gamma}\left(\left.E\right|_{U}\right)$. Assim, podemos identificar $\Gamma$ com a seção diferenciável $x \longmapsto \Gamma_{x}$ do fibrado vetorial $\operatorname{Lin}\left(\left.T M\right|_{U},\left.E\right|_{U} ;\left.E\right|_{U}\right)$. Por outro lado, podemos identificar o fibrado $\operatorname{Lin}\left(\left.T M\right|_{U},\left.E\right|_{U} ;\left.E\right|_{U}\right)$ com o fibrado vetorial $\operatorname{Lin}\left(\left.T M\right|_{U}, \operatorname{Lin}\left(\left.E\right|_{U}\right)\right)$, $\operatorname{logo} \Gamma$ pode ser identificado com um campo 1-tensorial diferenciável em $U$ à valores em $\operatorname{Lin}\left(\left.E\right|_{U}\right)$. Ou seja, para cada $x \in U$ e $v \in T_{x} M$, escrevemos

$$
\Gamma_{x}(v)=\Gamma_{x}(v, \cdot) \in \mathfrak{g l}\left(E_{x}\right),
$$

de modo que $\Gamma_{x}: T_{x} M \rightarrow \mathfrak{g l}\left(E_{x}\right)$ é uma aplicação linear.

Explicitamente, o tensor de Christoffel da conexão $\nabla$ em relação à seção local diferenciável $s: U \rightarrow \operatorname{FR}(E)$ é a aplicação $\mathrm{C}^{\infty}(M)$-bilinear tal que:

$$
\nabla_{v} \epsilon=s(x) \cdot \mathrm{d} \tilde{\epsilon}_{x}(v)+\Gamma_{x}(v) \cdot \epsilon(x),
$$

para quaisquer $x \in U, v \in T_{x} M$ e $\epsilon \in \boldsymbol{\Gamma}\left(\left.E\right|_{U}\right)$, onde $\tilde{\epsilon}: U \rightarrow \mathbb{R}^{n}$ denota a representação de $\epsilon$ em relação a $s$.

Teorema 2.4.10 Seja $\nabla$ uma conexão em $E$ e $s: U \rightarrow \operatorname{FR}(E)$ uma seção local diferenciável de $\operatorname{FR}(E)$. Então, existe uma única conexão $\operatorname{Hor}(\operatorname{FR}(E))$ em $\operatorname{FR}(E)$ tal que

$$
\Gamma_{x}(v)=\operatorname{Ad}_{s(x)}\left(\bar{\omega}_{x}(v)\right),
$$

para quaisquer $x \in U$ e $v \in T_{x} M$, onde $\bar{\omega}=s^{*} \omega$ e $\omega$ é a forma de conexão associada à conexão $\operatorname{Hor}(\operatorname{FR}(E))$. 
A prova do Teorema 2.4.10 passa pela construção do fibrado associado do $\operatorname{GL}\left(\mathbb{R}^{n}\right)$-fibrado principal $\mathrm{FR}(E)$. Como nosso objetivo não se estende a tais construções, reportamos o leitor a [27, Seção 2.5] ou [17, Seção 3.3]. A conexão $\operatorname{Hor}(\mathrm{FR}(E))$, dada pelo Teorema 2.4.10, é chamada a conexão correspondente à conexão $\nabla$.

Sejam $\left(E, \pi, M, \mathbb{R}^{n}\right)$ um fibrado vetorial e $\nabla$ uma conexão em $E$.

Definição 2.4.11 O tensor de curvatura da conexão $\nabla$ é a aplicação

$$
R: \boldsymbol{\Gamma}(T M) \times \boldsymbol{\Gamma}(T M) \times \boldsymbol{\Gamma}(E) \rightarrow \boldsymbol{\Gamma}(E)
$$

definida por

$$
R(X, Y) \epsilon=\nabla_{X} \nabla_{Y} \epsilon-\nabla_{Y} \nabla_{X} \epsilon-\nabla_{[X, Y]} \epsilon
$$

para quaisquer $X, Y \in \boldsymbol{\Gamma}(T M)$ e $\epsilon \in \boldsymbol{\Gamma}(E)$, onde $[X, Y] \in \boldsymbol{\Gamma}(T M)$ denota o colchete de Lie de $X$ e $Y$.

O tensor de curvatura $R$ é uma aplicação $\mathrm{C}^{\infty}(M)$-trilinear e, assim, para cada $x \in M, R$ define uma aplicação trilinear

$$
R_{x}: T_{x} M \times T_{x} M \times E_{x} \rightarrow E_{x} .
$$

Além disso, $R$ é anti-simétrico em relação às duas primeiras variáveis.

Definição 2.4.12 Seja $\iota: T M \rightarrow E$ um morfismo de fibrados vetoriais. O $\iota$-tensor de torção da conexão $\nabla$ é a aplicação

$$
T^{\iota}: \boldsymbol{\Gamma}(T M) \times \boldsymbol{\Gamma}(T M) \rightarrow \boldsymbol{\Gamma}(E)
$$

definida por

$$
T^{\iota}(X, Y)=\nabla_{X} \iota(Y)-\nabla_{Y} \iota(X)-\iota([X, Y]),
$$

para quaisquer $X, Y \in \boldsymbol{\Gamma}(T M)$.

Quando $E=T M$ e $\iota$ é a aplicação identidade então $T=T^{\iota}$ coincide com o tensor de torção usual de $\nabla$. O $\iota$-tensor de torção $T^{\iota}$ é uma aplicação $\mathrm{C}^{\infty}(M)$-bilinear e, assim, para cada $x \in M, T^{\iota}$ define uma aplicação bilinear

$$
T_{x}^{\iota}: T_{x} M \times T_{x} M \rightarrow E_{x} .
$$

Além disso, $T^{\iota}$ é anti-simétrico. 


\subsubsection{Conexão no fibrado vetorial quociente}

Sejam $\left(E, \pi, M, \mathbb{R}^{n}\right)$ um fibrado vetorial e $(F, \pi, M, V)$ um subfibrado vetorial. Para cada $x \in M$, o espaço vetorial $E_{x} / F_{x}$ é isomorfo a $\mathbb{R}^{n} / V$. Denotemos por

$$
E / F=\bigcup_{x \in M} E_{x} / F_{x}
$$

e por $\pi$ a aplicação que transforma $E_{x} / F_{x}$ sobre $x$, para todo $x \in M$. Mostremos que $\left(E / F, \pi, M, \mathbb{R}^{n} / V\right)$ é um fibrado vetorial. Seja $s: U \rightarrow$ $\operatorname{FR}(E)$ uma seção local diferenciável. Para cada $x \in U$, denotemos por $\bar{s}(x): \mathbb{R}^{n} / V \rightarrow E_{x} / F_{x}$ a (única) aplicação linear que torna o diagrama

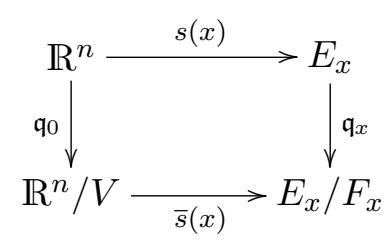

comutativo, onde $\mathfrak{q}_{0}$ e $\mathfrak{q}_{x}$ denotam as respectivas aplicações quocientes. O diagrama (2.41) define, portanto, uma seção local $\bar{s}: U \rightarrow \mathrm{FR}(E / F)$ de $\operatorname{FR}(E / F)$. Sejam agora $s_{1}: U_{1} \rightarrow \operatorname{FR}(E)$ e $s_{2}: U_{2} \rightarrow \mathrm{FR}(E)$ seções locais diferenciáveis com aplicação de transição $g: U_{1} \cap U_{2} \rightarrow \mathrm{GL}\left(\mathbb{R}^{n}\right)$. Então, a aplicação de transição

$$
\bar{g}: U_{1} \cap U_{2} \rightarrow \mathrm{GL}\left(\mathbb{R}^{n} / V\right)
$$

de $\bar{s}_{1}: U_{1} \rightarrow \mathrm{FR}(E / F)$ para $\bar{s}_{2}: U_{2} \rightarrow \mathrm{FR}(E / F)$ é dada por $\bar{g}=\overline{\mathfrak{q}}_{0} \circ g$, onde $\overline{\mathfrak{q}}_{0}: \mathrm{GL}\left(\mathbb{R}^{n}\right) \rightarrow \mathrm{GL}\left(\mathbb{R}^{n} / V\right)$ denota a aplicação quociente. Como $\overline{\mathfrak{q}}_{0}$ e $g$ são diferenciáveis segue que $\bar{g}$ é uma aplicação diferenciável. Assim, o conjunto

$$
\mathcal{A}=\{\bar{s}: s \in \boldsymbol{\Gamma}(\mathrm{FR}(E))\}
$$

define um atlas de seções locais de $\operatorname{FR}(E / F)$. Considerando $\mathcal{A}_{\max }$ o único atlas maximal de seções locais de $\mathrm{FR}(E / F) \rightarrow M$ contendo (2.42), segue que $\left(E / F, \pi, M, \mathbb{R}^{n} / V\right)$ é um fibrado vetorial, chamado o fibrado vetorial quociente de $E$ por $F$.

Exemplo 2.4.13 Sejam $\left(E, \pi, M, \mathbb{R}^{n}\right)$ um fibrado vetorial, $\nabla$ uma conexão em $E$ e $F$ um subfibrado vetorial de $E$. Denotando por $\mathfrak{q}: E \rightarrow E / F$ a aplicação quociente, segue que a aplicação

$$
(X, \epsilon) \in \boldsymbol{\Gamma}(T M) \times \boldsymbol{\Gamma}(F) \longmapsto \mathfrak{q} \circ \nabla_{X} \epsilon \in \boldsymbol{\Gamma}(E / F)
$$


é $\mathrm{C}^{\infty}(M)$-bilinear. Assim, pelo Lema 2.2.14, existe uma seção diferenciável $\alpha^{F}$ de $\operatorname{Lin}(T M, F ; E / F)$ tal que

$$
\nabla_{v} \epsilon+F_{x}=\alpha_{x}^{F}(v, \epsilon(x)) \in E_{x} / F_{x}
$$

para quaisquer $x \in M$ e $v \in T_{x} M$. A seção $\alpha^{F}$ é chamada a segunda forma fundamental do subfibrado vetorial $F$.

\subsection{A forma de curvatura}

O objetivo desta seção é explicitar a relação existente entre o tensor de curvatura $R$ de uma conexão $\nabla$ em um fibrado vetorial $E$ e a forma de conexão $\Omega$, que é uma 2-forma diferenciável associada à forma de conexão $\omega$ de $\nabla$. Faremos isso sem demonstrações, apenas enunciando o resultado principal.

Seja $P(M, \Pi, G)$ um fibrado principal munido de uma conexão $\operatorname{Hor}(P)$. Denotemos por $\omega$ a forma de conexão de $\operatorname{Hor}(P)$. Dado uma $k$-forma diferencial $\lambda$ em $P$, denotemos por $\mathrm{d} \lambda$ sua diferencial exterior ${ }^{6}$, que é uma $(k+1)$-forma diferencial em $P$.

Definição 2.5.1 Seja $\lambda$ uma $k$-forma diferencial em $P$. A diferencial exterior covariante de $\lambda$ é a $(k+1)$-forma $D \lambda$ em $P$ definida por:

$$
D \lambda_{p}\left(\zeta_{1}, \ldots, \zeta_{k+1}\right)=\mathrm{d} \lambda_{p}\left(\pi_{\mathrm{hor}}\left(\zeta_{1}\right), \ldots, \pi_{\mathrm{hor}}\left(\zeta_{k+1}\right)\right),
$$

para quaisquer $p \in P$ e $\zeta_{1}, \ldots, \zeta_{k+1} \in T_{p} P$.

Claramente a diferencial exterior covariante de uma $k$-forma diferenciável é uma $(k+1)$-forma diferenciável em $P$.

Definição 2.5.2 A forma de curvatura da conexão $\operatorname{Hor}(P)$ é a 2-forma diferenciável em $P$ à valores em $\mathfrak{g}$ definida por:

$$
\Omega=D \omega .
$$

Proposição 2.5.3 A forma de curvatura $\Omega$ é dada por:

$$
\Omega=\mathrm{d} \omega+\frac{1}{2} \omega \wedge \omega
$$

\footnotetext{
${ }^{6}$ Aqui estamos supondo que $\lambda$ é diferenciável.
} 
onde o produto wedge é considerado em relação ao colchete de Lie de $\mathfrak{g}$. De forma mais explícita, (2.43) siginifica:

$$
\Omega_{p}\left(\zeta_{1}, \zeta_{2}\right)=\mathrm{d} \omega_{p}\left(\zeta_{1}, \zeta_{2}\right)+\left[\omega_{p}\left(\zeta_{1}\right), \omega_{p}\left(\zeta_{2}\right)\right],
$$

para quaisquer $p \in P$ e $\zeta_{1}, \zeta_{2} \in T_{p} P$.

Demonstração. Ver [17, Teorema 2.5.2].

Seja ( $\left.E, \pi, M, \mathbb{R}^{n}\right)$ um fibrado vetorial munido de uma conexão $\nabla$. O tensor de curvatura $R$ de $\nabla$ pode ser identificado com uma 2-forma diferenciável em $M$ à valores em $\mathfrak{g l}\left(\mathbb{R}^{n}\right)$. Mais precisamente, temos o seguinte:

Lema 2.5.4 Seja $\left(E, \pi, M, \mathbb{R}^{n}\right)$ um fibrado vetorial munido de uma conexão $\nabla$. A forma de curvatura $\Omega$ correspondente à conexão no $\mathrm{GL}\left(\mathbb{R}^{n}\right)$-fibrado principal $\mathrm{FR}(E)$ está associada ao tensor de curvatura $R$ de $\nabla$; de forma mais explícita:

$$
p \circ \Omega_{p}\left(\zeta_{1}, \zeta_{2}\right) \circ p^{-1}=R_{x}\left(\mathrm{~d} \Pi_{p}\left(\zeta_{1}\right), \mathrm{d} \Pi_{p}\left(\zeta_{2}\right)\right) \in \operatorname{Lin}\left(E_{x}\right),
$$

para quaisquer $x \in M, p \in \operatorname{FR}\left(E_{x}\right)$ e $\zeta_{1}, \zeta_{2} \in T_{p} \operatorname{FR}(E)$, onde

$$
\Pi: \operatorname{FR}(E) \rightarrow M
$$

denota a projeção.

Demonstração. Ver Seção 3.5 de [17].

\subsection{Conexões lineares $G$-invariantes em espaços ho- mogêneos}

Seja $G / H$ um espaço homogêneo, onde $G$ é um grupo de Lie e $H$ é um subgrupo fechado de $G$. Uma conexão linear $\nabla$ em $G / H$ é chamada $G$ invariante se, para todo $g \in G$, o difeomorfismo $\bar{L}_{g}: G / H \rightarrow G / H$, definido em (1.46), é um difeomorfismo afim. Por exemplo, seja $\langle\cdot, \cdot\rangle$ uma métrica semi-Riemanniana $G$-invariante em $G / H$. Então, a conexão de Levi-Civita de $(G / H,\langle\cdot, \cdot\rangle)$ é $G$-invariante.

Nesta seção discutiremos uma caracterização das conexões $G$-invariantes em $G / H$ em termos de aplicações lineares $\lambda: \mathfrak{m} \rightarrow \mathfrak{g l}(\mathfrak{m})$, onde $\mathfrak{m}$ é um subespaço de $\mathfrak{g}$ tal que $\mathfrak{g}=\mathfrak{h} \oplus \mathfrak{m}$. Identificaremos, assim, o espaço tangente $T_{1 H}(G / H)$ com $\mathfrak{m}$ de maneira natural.

Denotemos por $T(G / H)$ o fibrado tangente de $G / H$ e consideremos o $\mathrm{GL}(\mathfrak{m})$-fibrado principal $\mathrm{FR}_{\mathfrak{m}}(T(G / H))$. 
Observação 2.6.1 Aqui, estamos considerando o fibrado tangente $T(G / H)$ com fibra típica $\mathfrak{m}$ ao invés de $\mathbb{R}^{n}$. Isso é feito como segue: escolha um isomorfismo linear $\mathfrak{i}: \mathfrak{m} \rightarrow \mathbb{R}^{n}$ e considere a aplicação

$$
\gamma_{\mathfrak{i}}: \operatorname{FR}(T(G / H)) \rightarrow \mathrm{FR}_{\mathfrak{m}}(T(G / H))
$$

definida por $\gamma_{\mathfrak{i}}(p)=p \circ \mathfrak{i}$, para todo $p \in \operatorname{FR}(T(G / H))$. Usando o Lema 2.1.8, é possível mostrar que existe um único atlas maximal de seções locais de $\mathrm{FR}_{\mathfrak{m}}(T(G / H)) \rightarrow M$ que torna $\gamma_{\mathfrak{i}}$ um isomorfismo de fibrados principais, cujo homomorfismo subjacente é

$$
\mathcal{I}_{\mathfrak{i}}: \mathrm{GL}(\mathfrak{m}) \rightarrow \mathrm{GL}\left(\mathbb{R}^{n}\right),
$$

definido por $\mathcal{I}_{\mathfrak{i}}(T)=\mathfrak{i} \circ T \circ \mathfrak{i}^{-1}$, para todo $T \in \mathrm{GL}(\mathfrak{m})$. Tal atlas maximal não depende da escolha do isomorfismo linear $\mathfrak{i}: \mathfrak{m} \rightarrow \mathbb{R}^{n}$. Além disso, a estrutura diferenciável de $T(G / H)$ não muda quando mudamos a fibra típica de $\mathbb{R}^{n}$ para $\mathfrak{m}$.

Temos uma ação diferenciável à esquerda de $G$ em $\operatorname{FR}_{\mathfrak{m}}(T(G / H))$ definida por:

$$
g \cdot p=\mathrm{d} \bar{L}_{g}(x) \circ p
$$

para quaisquer $x \in G / H, p \in \mathrm{FR}_{\mathfrak{m}}\left(T_{x}(G / H)\right)$ e $g \in G$. Podemos, assim, definir uma ação diferenciável à esquerda de $G \times \mathrm{GL}(\mathfrak{m})$ em $\mathrm{FR}_{\mathfrak{m}}(T(G / H))$ como sendo:

$$
(g, \tau) \cdot p=(g \cdot p) \circ \tau^{-1}=g \cdot\left(p \circ \tau^{-1}\right),
$$

para quaisquer $p \in \mathrm{FR}_{\mathfrak{m}}(T(G / H)), g \in G$ e $\tau \in \mathrm{GL}(\mathfrak{m})$. Denotemos por

$$
\operatorname{Id}_{\mathfrak{m}} \in \mathrm{FR}_{\mathfrak{m}}\left(T_{1 H}(G / H)\right)
$$

a aplicação identidade de $\mathfrak{m}$ e seja $S \subset G \times \mathrm{GL}(\mathfrak{m})$ o subgrupo de isotropia de $\operatorname{Id}_{\mathfrak{m}}$. A ação (2.46) é transitiva e, portanto, temos um difeomorfismo diferenciável:

$$
\Upsilon:(G \times \mathrm{GL}(\mathfrak{m})) / S \rightarrow \mathrm{FR}_{\mathfrak{m}}(T(G / H))
$$

dado por

$$
\Upsilon((g, \tau) S)=\mathrm{d} \bar{L}_{g}(1 H) \circ \tau^{-1}
$$


O difeomorfismo (2.47) define, portanto, um diagrama comutativo:

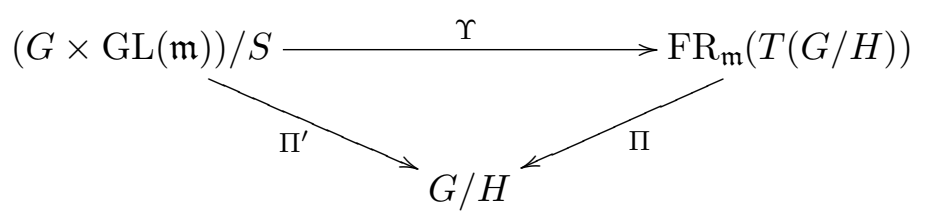

onde $\Pi^{\prime}$ é definida por

$$
\Pi^{\prime}((g, \tau) S)=g H
$$

Usando (1.56), obtemos:

$$
S=\operatorname{Gr}(\overline{\mathrm{Ad}})=\left\{\left(h, \overline{\operatorname{Ad}}_{h}\right): h \in H\right\} \subset H \times \mathrm{GL}(\mathfrak{m}) .
$$

A álgebra de Lie de $S$ é portanto dada por:

$$
\mathfrak{s}=\operatorname{Gr}(\overline{\operatorname{ad}})=\left\{\left(X, \overline{\operatorname{ad}}_{X}\right): X \in \mathfrak{h}\right\} \subset \mathfrak{h} \oplus \mathfrak{g l}(\mathfrak{m}) .
$$

Uma conexão em $G / H$ é unicamente determinada por uma conexão $\operatorname{Hor}\left(\mathrm{FR}_{\mathfrak{m}}(T(G / H))\right)$ no fibrado principal $\mathrm{FR}_{\mathfrak{m}}(T(G / H))$. Denotemos por $\mathcal{D}$ a distribuição diferenciável no quociente $(G \times \mathrm{GL}(\mathfrak{m})) / S$ que corresponde a $\operatorname{Hor}\left(\mathrm{FR}_{\mathfrak{m}}(T(G / H))\right)$ através do difeomorfismo $\Upsilon$. Uma conexão em $G / H$ é $G$-invariante se, e somente se, a conexão correspondente em $\mathrm{FR}_{\mathfrak{m}}(T(G / H))$ é $G$-invariante. Assim, as conexões $G$-invariantes em $G / H$ estão em correspondência biunívoca com as distribuições diferenciáveis $\mathrm{GL}(\mathfrak{m})$-invariantes $\mathcal{D}$ em $(G \times \mathrm{GL}(\mathfrak{m})) / S$ que são horizontais em relação a $\Pi^{\prime}$.

De (2.49) segue que $\mathfrak{m} \oplus \mathfrak{g l}(\mathfrak{m})$ é um complemento de $\mathfrak{s}$ no espaço $\mathfrak{g} \oplus \mathfrak{g l}(\mathfrak{m})$. Assim, a diferencial da aplicação quociente

$$
(g, \tau) \in G \times \mathrm{GL}(\mathfrak{m}) \longmapsto(g, \tau) S \in(G \times \mathrm{GL}(\mathfrak{m})) / S
$$

no ponto $\left(1, \operatorname{Id}_{\mathfrak{m}}\right)$ restringe-se a um isomorfismo de $\mathfrak{m} \oplus \mathfrak{g l}(\mathfrak{m})$ ao espaço tangente de $(G \times \mathrm{GL}(\mathfrak{m})) / S$ no ponto $\left(1, \operatorname{Id}_{\mathfrak{m}}\right) S$; identificaremos então este espaço tangente com $\mathfrak{m} \oplus \mathfrak{g l}(\mathfrak{m})$ através deste isomorfismo. Consideremos a representação isotrópica

$$
\overline{\mathrm{Ad}}: S \rightarrow \mathrm{GL}(\mathfrak{m} \oplus \mathfrak{g l}(\mathfrak{m}))
$$

de $S$ em $\mathfrak{m} \oplus \mathfrak{g l}(\mathfrak{m})$. Uma distribuição $(G \times \mathrm{GL}(\mathfrak{m}))$-invariante $\mathcal{D}$ em $(G \times$ $\mathrm{GL}(\mathfrak{m})) / S$ é unicamente determinada ${ }^{7}$ por um subespaço $\mathfrak{d}=\mathcal{D}_{\left(1, \operatorname{Id}_{\mathfrak{m}}\right) S}$ de

\footnotetext{
${ }^{7}$ Esta afirmação é a versão mais geral do Lema 1.6.7; ver [17] para maiores detalhes.
} 
$\mathfrak{m} \oplus \mathfrak{g l}(\mathfrak{m})$ que é invariante sob a representação isotrópica (2.50). A representação isotrópica (2.50) é dada por:

$$
\overline{\operatorname{Ad}}_{s}(X, \kappa)=\left(\overline{\operatorname{Ad}}_{h}(X), \overline{\operatorname{Ad}}_{h} \circ \kappa \circ \overline{\operatorname{Ad}}_{h}^{-1}-\overline{\operatorname{ad}}_{\mathrm{pr}_{\mathfrak{h}} \operatorname{Ad}_{h}(X)}\right),
$$

para quaisquer $X \in \mathfrak{m}, \kappa \in \mathfrak{g l}(\mathfrak{m}), h \in H$, onde $s=\left(h, \overline{\operatorname{Ad}}_{h}\right) \in S$. A diferencial

$$
\overline{\mathrm{ad}}: \mathfrak{s} \rightarrow \mathfrak{g l}(\mathfrak{m} \oplus \mathfrak{g l}(\mathfrak{m}))
$$

de (2.50) é dada por:

$$
\overline{\operatorname{ad}}_{\sigma}(X, \kappa)=\left(\overline{\operatorname{ad}}_{Y}(X),\left[\overline{\operatorname{ad}}_{Y}, \kappa\right]-\overline{\operatorname{ad}}_{\mathrm{pr}_{\mathfrak{h}}[Y, X]}\right),
$$

para quaisquer $X \in \mathfrak{m}, \kappa \in \mathfrak{g l}(\mathfrak{m}), Y \in \mathfrak{h}$, onde $\sigma=\left(Y, \overline{\operatorname{ad}}_{Y}\right) \in \mathfrak{s}$. A diferencial da aplicação $\Pi^{\prime}:(G \times \mathrm{GL}(\mathfrak{m})) / S \rightarrow G / H$ no ponto $\left(1, \operatorname{Id}_{\mathfrak{m}}\right) S$ é dada por:

$$
(X, \kappa) \in \mathfrak{m} \oplus \mathfrak{g l}(\mathfrak{m}) \longmapsto X \in \mathfrak{m} .
$$

Assim, $\mathcal{D}$ é horizontal em relação a $\Pi^{\prime}$ se, e somente se, $\mathfrak{d}$ é um complemento de $\mathfrak{g l}(\mathfrak{m})$ em $\mathfrak{m} \oplus \mathfrak{g l}(\mathfrak{m})$. Temos, assim, o seguinte:

Lema 2.6.2 Existe uma correspondência biunívoca entre as conexões $G$ invariantes em $G / H$ e as aplicações lineares $\lambda: \mathfrak{m} \rightarrow \mathfrak{g l}(\mathfrak{m})$ que satisfazem a seguinte condição:

$$
\overline{\operatorname{Ad}}_{h} \circ \lambda(X) \circ \overline{\operatorname{Ad}}_{h}^{-1}-\overline{\operatorname{ad}}_{\operatorname{pr}_{\mathfrak{h}} \operatorname{Ad}_{h}(X)}=\lambda\left(\overline{\operatorname{Ad}}_{h}(X)\right),
$$

para quaisquer $h \in H$ e $X \in \mathfrak{m}$. A condição (2.54) implica:

$$
\left[\overline{\operatorname{ad}}_{Y}, \lambda(X)\right]-\overline{\operatorname{ad}}_{\operatorname{pr}_{\mathfrak{h}}[Y, X]}=\lambda\left(\overline{\operatorname{ad}}_{Y}(X)\right),
$$

para quaisquer $X \in \mathfrak{m}$ e $Y \in \mathfrak{h}$. Se $H$ é conexo então a condição (2.54) é equivalente à condição $(2.55)$.

Demonstração. Um subespaço $\mathfrak{d}$ de $\mathfrak{m} \oplus \mathfrak{g l}(\mathfrak{m})$ é um complemento de $\mathfrak{g l}(\mathfrak{m})$ se, e somente se, $\mathfrak{d}$ é o gráfico de uma aplicação linear $\lambda: \mathfrak{m} \rightarrow \mathfrak{g l}(\mathfrak{m})$. Usando (2.51), segue que $(G r)(\lambda)$ é invariante sob a representação isotrópica (2.50) se, e somente se, a condição (2.54) é satisfeita. O restante da prova segue diretamente de (2.52) e do Lema 1.3.1.

Explicitaremos agora o tensor de curvatura $R$ de uma conexão $G$-invariante $\nabla$ em $G / H$ em termos da correspondente aplicação linear $\lambda$. O tensor 
de torção $T$ de $\nabla$ pode ser determinado de maneira similar; no entanto, como estamos interessados no caso em que $\nabla$ é a conexão de Levi-Civita de alguma métrica semi-Riemanniana $G$-invariante em $G / H$, explicitaremos somente o tensor de curvatura.

Seja $\nabla$ uma conexão $G$-invariante em $G / H$. Como $\mathfrak{q}: G \rightarrow G / H$ é um $H$-fibrado principal (cf. Exemplo 2.1.5), consideremos o morfismo de fibrados principais:

$$
\phi: G \rightarrow \mathrm{FR}_{\mathfrak{m}}(T(G / H))
$$

definido por:

$$
\phi(g)=\mathrm{d} \bar{L}_{g}(1 H)
$$

para todo $g \in G$, cujo homomorfismo subjacente é a representação isotrópica $\overline{\mathrm{Ad}}: H \rightarrow \mathrm{GL}(\mathfrak{m})$. Seja $\omega$ a forma de conexão de $\nabla$ em $\mathrm{FR}_{\mathfrak{m}}(T(G / H))$. Como o difeomorfismo $\bar{L}_{g}: G / H \rightarrow G / H$ é uma aplicação afim, para cada $g \in G, \omega$ é invariante pela ação de $G$ em $\mathrm{FR}_{\mathfrak{m}}(T(G / H)$ ) (cf. (2.45)). Segue então que a forma diferencial $\phi^{*} \omega$ em $G$ é invariante à esquerda. De (2.43), temos:

$$
\begin{aligned}
\left(\phi^{*} \Omega\right)_{1}(X, Y) & =\mathrm{d}\left(\phi^{*} \omega\right)_{1}(X, Y)+\frac{1}{2}\left(\left(\phi^{*} \omega\right)_{1} \wedge\left(\phi^{*} \omega\right)_{1}\right)(X, Y) \\
& =-\left(\phi^{*} \omega\right)_{1}([X, Y])+\left[\left(\phi^{*} \omega\right)_{1}(X),\left(\phi^{*} \omega\right)_{1}(Y)\right]
\end{aligned}
$$

para quaisquer $X, Y \in \mathfrak{g}$.

Lema 2.6.3 A forma invariante à esquerda $\phi^{*} \omega$ é dada por:

$$
\left(\phi^{*} \omega\right)_{1}(X)=\lambda\left(\operatorname{pr}_{\mathfrak{m}}(X)\right)+\overline{\operatorname{ad}}_{\mathrm{pr}_{\mathfrak{h}}(X)},
$$

para todo $X \in \mathfrak{g}$, onde $\lambda: \mathfrak{m} \rightarrow \mathfrak{g l}(\mathfrak{m})$ é a aplicação linear correspondente à conexão $\nabla$ dada no Lema 2.6.2.

Demonstração. Inicialmente, calcularemos $\Upsilon^{*} \omega$, onde $\Upsilon$ é o difeomorfismo dado em (2.47). Consideremos o difeomorfismo:

$$
\beta_{\mathrm{Id}_{\mathfrak{m}}}: \mathrm{GL}(\mathfrak{m}) \rightarrow \mathrm{FR}_{\mathfrak{m}}\left(T_{1 H}(G / H)\right)
$$

dado pela ação no elemento $\operatorname{Id}_{\mathfrak{m}}$. A diferencial de $\beta_{\operatorname{Id}_{\mathfrak{m}}}$ em $\operatorname{Id}_{\mathfrak{m}} \in \mathrm{GL}(\mathfrak{m})$ é um isomorfismo:

$$
\mathrm{d} \beta_{\operatorname{Id}_{\mathfrak{m}}}\left(\operatorname{Id}_{\mathfrak{m}}\right): \mathfrak{g l}(\mathfrak{m}) \rightarrow \operatorname{Ver}_{\operatorname{Id}_{\mathfrak{m}}}\left(\operatorname{FR}_{\mathfrak{m}}\left(T_{1 H}(G / H)\right)\right) .
$$


A restrição de $\omega$ a $\operatorname{Ver}_{\operatorname{Id}_{\mathfrak{m}}}\left(\mathrm{FR}_{\mathfrak{m}}\left(T_{1 H}(G / H)\right)\right)$ é a inversa de (2.59). O isomorfismo

$$
\mathrm{d} \Upsilon\left(\left(1, \operatorname{Id}_{\mathfrak{m}}\right) S\right): \mathfrak{m} \oplus \mathfrak{g l}(\mathfrak{m}) \rightarrow T_{\operatorname{Id}_{\mathfrak{m}}} \operatorname{FR}_{\mathfrak{m}}\left(T_{1 H}(G / H)\right)
$$

transforma $\mathfrak{g l}(\mathfrak{m})$ (que é o núcleo de $(2.53)$ ) sobre $\operatorname{Ver}_{\operatorname{Id}_{\mathfrak{m}}}\left(\operatorname{FR}_{\mathfrak{m}}\left(T_{1 H}(G / H)\right)\right.$ ). A restrição de $\Upsilon^{*} \omega$ a $\mathfrak{g l}(\mathfrak{m})$ é igual a composição da restrição de $(2.60)$ a $\mathfrak{g l}(\mathfrak{m})$ com a inversa de (2.59). Tal composição é a diferencial no ponto $\left(1, \operatorname{Id}_{\mathfrak{m}}\right) S$ da aplicação

$$
\beta_{\mathrm{Id}_{\mathfrak{m}}}^{-1} \circ \Upsilon: \Pi^{\prime-1}(1 H)=(H \times \mathrm{GL}(\mathfrak{m})) / S \rightarrow \mathrm{GL}(\mathfrak{m})
$$

dada por

$$
(h, \tau) S \longmapsto \overline{\operatorname{Ad}}_{h} \circ \tau^{-1} .
$$

Isso é calculado facilmente como:

$$
\left(\Upsilon^{*} \omega\right)_{(1, \mathrm{Id}) S}(\kappa)=-\kappa \in \mathfrak{g l}(\mathfrak{m}),
$$

para todo $\kappa \in \mathfrak{g l}(\mathfrak{m})$. A aplicação (2.60) transforma o gráfico de $\lambda, \operatorname{Gr}(\lambda)$, sobre $\operatorname{Hor}_{I_{\mathfrak{m}}}\left(\mathrm{FR}_{\mathfrak{m}}\left(T_{1 H}(G / H)\right)\right)$ e, assim, $\Upsilon^{*} \omega$ anula-se em $\operatorname{Gr}(\lambda)$. Assim, temos:

$$
\left(\Upsilon^{*} \omega\right)_{(1, \mathrm{Id}) S}(X, \kappa)=\lambda(X)-\kappa \in \mathfrak{g l}(\mathfrak{m}),
$$

para quaisquer $X \in \mathfrak{m}$ e $\kappa \in \mathfrak{g l}(\mathfrak{m})$. Calculemos agora $\phi^{*} \omega$. Temos:

$$
\phi^{*} \omega=\left(\Upsilon^{-1} \circ \phi\right)^{*} \Upsilon^{*} \omega,
$$

onde

$$
\Upsilon^{-1} \circ \phi: G \rightarrow(G \times \mathrm{GL}(\mathfrak{m})) / S
$$

é dada por $\left(\Upsilon^{-1} \circ \phi\right)(g)=\left(g, \operatorname{Id}_{\mathfrak{m}}\right) S$, para todo $g \in G$. A diferencial da aplicação $\Upsilon^{-1} \circ \phi$ no elemento identidade $1 \in G$ é dada por:

$$
X \in \mathfrak{g} \longmapsto\left(\operatorname{pr}_{\mathfrak{m}}(X),-\overline{\operatorname{ad}}_{\operatorname{pr}(X)}\right) \in \mathfrak{m} \oplus \mathfrak{g l}(\mathfrak{m}) .
$$

A conclusão segue agora de (2.61), (2.62) e (2.63).

Corolário 2.6.4 A forma invariante à esquerda $\phi^{*} \Omega$ é dada por:

$$
\begin{aligned}
\left(\phi^{*} \Omega\right)_{1}(X, Y)= & -\left(\lambda \circ \operatorname{pr}_{\mathfrak{m}}\right)([X, Y])-\overline{\operatorname{ad}}_{\operatorname{pr}_{\mathfrak{h}}[X, Y]} \\
& +\left[\lambda\left(\operatorname{pr}_{\mathfrak{m}}(X)\right)+\overline{\operatorname{ad}}_{p_{\mathfrak{h}}(X)}, \lambda\left(\operatorname{pr}_{\mathfrak{m}}(Y)\right)+\overline{\operatorname{ad}}_{\operatorname{pr}_{\mathfrak{h}}(Y)}\right],
\end{aligned}
$$

para quaisquer $X, Y \in \mathfrak{g}$. 
Demonstração. Segue diretamente de (2.57) e do Lema 2.6.3.

Proposição 2.6.5 Seja $\nabla$ uma conexão $G$-invariante em $G / H$ correspondendo à uma aplicação linear $\lambda: \mathfrak{m} \rightarrow \mathfrak{g l}(\mathfrak{m})$, como no Lema 2.6.2. Então, o tensor de curvatura $R$ de $\nabla$ no ponto $1 H \in G / H$ é dado por:

$$
R_{1 H}(X, Y)=[\lambda(X), \lambda(Y)]-\overline{\operatorname{ad}}_{\operatorname{pr}_{\mathfrak{h}}[X, Y]}-\left(\lambda \circ \operatorname{pr}_{\mathfrak{m}}\right)([X, Y]),
$$

para quaisquer $X, Y \in \mathfrak{m}$, onde identificamos $\mathfrak{m} \operatorname{com} T_{1 H}(G / H)$.

Demonstração. Pelo Lema 2.5.4, temos:

$$
R_{1 H}(X, Y)=\Omega\left(\zeta_{1}, \zeta_{2}\right),
$$

onde $\zeta_{1}, \zeta_{2} \in T_{\operatorname{Id}_{\mathfrak{m}}} \mathrm{FR}_{\mathfrak{m}}\left(T_{1 H}(G / H)\right)$ são tais que:

$$
\mathrm{d} \Pi\left(\zeta_{1}\right)=X \quad \text { e } \quad \mathrm{d} \Pi\left(\zeta_{2}\right)=Y .
$$

Se $\phi$ denota o morfismo de fibrados principais definido em (2.56), então $\zeta_{1}=\mathrm{d} \phi_{1}(X)$ e $\zeta_{2}=\mathrm{d} \phi_{1}(Y)$ satisfazem (2.66). Assim,

$$
R_{1 H}(X, Y)=\left(\phi^{*} \Omega\right)_{1}(X, Y),
$$

para quaisquer $X, Y \in \mathfrak{m}$. A conclusão segue então do Corolário 2.6.4.

\subsubsection{A conexão de Levi-Civita}

Seja $\langle\cdot, \cdot\rangle$ uma métrica semi-Riemanniana $G$-invariante em $G / H$ e seja $\nabla$ a conexão de Levi-Civita de $(G / H,\langle\cdot, \cdot\rangle)$. A conexão $\nabla$, por ser $G$-invariante, corresponde à uma aplicação linear $\lambda: \mathfrak{m} \rightarrow \mathfrak{g l}(\mathfrak{m})$, como no Lema 2.6.2. Determinaremos uma expressão para $\lambda$ em termos da métrica $\langle\cdot, \cdot\rangle$.

Observação 2.6.6 Considerando a decomposição $\mathfrak{g}=\mathfrak{h} \oplus \mathfrak{m}$, podemos considerar uma seção local diferenciável $s: U \subset G / H \rightarrow G$ da aplicação quociente $\pi: G \rightarrow G / H, \operatorname{com} 1 H \in U, s(1 H)=1$ e tal que $\mathrm{d} s(1 H): \mathfrak{m} \rightarrow \mathfrak{g}$ seja a aplicação inclusão de $\mathfrak{m}$ em $\mathfrak{g}$. Basta, por exemplo, considerar a inversa da restrição de $\pi$ à imagem pela aplicação exponencial de uma vizinhança suficientemente pequena de $0 \mathrm{em} \mathfrak{m}$.

Dado $X \in \mathfrak{g}$, definimos um campo vetorial diferenciável $\bar{X}$ em $U$ fazendo:

$$
\bar{X}(g H)=\mathrm{d} \bar{L}_{s(g H)}(1 H)\left(\mathrm{d} \pi_{1}(X)\right)
$$


para todo $g H \in U$. Como estamos identificando $T_{1 H}(G / H)$ com $\mathfrak{g} / \mathfrak{h}$, temos $\mathrm{d} \pi_{1}(X)=X+\mathfrak{h}$, para todo $X \in \mathfrak{g}$. Assim,

$$
\bar{X}(g H)=\mathrm{d} \bar{L}_{s(g H)}(1 H)(X+\mathfrak{h}) .
$$

Um cálculo simples mostra que, se $X, Y \in \mathfrak{m}$, então:

$$
[\bar{X}, \bar{Y}]_{1 H}=\overline{[X, Y]}_{1 H} \text {. }
$$

Como os campos vetoriais $\bar{X}$ são invariantes pela ação de $G$, segue da fórmula de Koszul que:

$$
\left\langle\nabla_{\bar{X}} \bar{Y}, \bar{Z}\right\rangle=\frac{1}{2}(\langle[\bar{X}, \bar{Y}], \bar{Z}\rangle-\langle[\bar{X}, \bar{Z}], \bar{Y}\rangle-\langle[\bar{Y}, \bar{Z}], \bar{X}\rangle) .
$$

Teorema 2.6.7 Sejam $\langle\cdot, \cdot\rangle$ uma métrica semi-Riemanniana $G$-invariante em $G / H$ e $\nabla$ a conexão de Levi-Civita de $(G / H,\langle\cdot, \cdot\rangle)$. Se $\lambda: \mathfrak{m} \rightarrow \mathfrak{g l}(\mathfrak{m})$ denota a aplicação linear dada no Lema 2.6.2, então $\lambda$ satisfaz:

$$
\begin{aligned}
\langle\lambda(X) Y, Z\rangle= & \frac{1}{2}\left(\left\langle\operatorname{pr}_{\mathfrak{m}}([X, Y]), Z\right\rangle-\left\langle\operatorname{pr}_{\mathfrak{m}}([X, Z]), Y\right\rangle\right. \\
& \left.-\left\langle\operatorname{pr}_{\mathfrak{m}}([Y, Z]), X\right\rangle\right)
\end{aligned}
$$

para quaisquer $X, Y, Z \in \mathfrak{m}$, onde $\langle\cdot, \cdot\rangle$ dado em (2.69) denota o produto escalar em $\mathfrak{m}$ correspondente à métrica $\langle\cdot, \cdot\rangle$, dado no Lema 1.6.7.

Demonstração. Seja $s_{0}: U \rightarrow G$ uma seção local diferenciável de $\pi$ como na Observação 2.6.6. Então,

$$
s=\phi \circ s_{0}: U \rightarrow \mathrm{FR}_{\mathfrak{m}}(T(G / H))
$$

é uma seção local diferenciável de $\mathrm{FR}_{\mathfrak{m}}(T(G / H))$, onde $\phi$ é o morfismo de fibrados principais dado em (2.56). Denotemos por $\Gamma$ o tensor de Christoffel da conexão $\nabla$ em relação a $s$. Dado um campo vetorial $\bar{X}$ como em (2.67), denotemos por $\widetilde{X}: U \rightarrow \mathfrak{m}$ a representação de $\bar{X}$ em relação a $s$ (cf. (2.16)). Temos:

$$
\begin{aligned}
\widetilde{X}(g H) & =s(g H)^{-1} \cdot \bar{X}(g H) \\
& =\left(\mathrm{d} \bar{L}_{s_{0}(g H)}(1 H)\right)^{-1} \cdot \mathrm{d} \bar{L}_{s_{0}(g H)}(1 H)(X+\mathfrak{h}) \\
& =X+\mathfrak{h} .
\end{aligned}
$$

Isso mostra que a representação $\widetilde{X}$ de $\bar{X}$ é constante e, portanto:

$$
\nabla_{v} \bar{Y}=\mathrm{dI}_{v}^{s} \bar{Y}+\Gamma_{g H}(v) \cdot \bar{Y}(g H)=\Gamma_{g H}(v) \cdot \bar{Y}(g H) .
$$


Em particular, se $X, Y \in \mathfrak{m}$, temos:

$$
\nabla_{X} \bar{Y}=\Gamma(X) Y
$$

Por outro lado, seja $\omega$ a forma de conexão de $\nabla$ em $\operatorname{FR}_{\mathfrak{m}}(T(G / H))$ e consideremos $\bar{\omega}=s^{*} \omega$ a representação de $\omega$ em relação a $s$. Temos $\bar{\omega}=s_{0}^{*}\left(\phi^{*} \omega\right)$ e, portanto:

$$
\bar{\omega}_{1 H}=\left(\phi^{*} \omega\right)_{1} \circ \mathrm{d} s(1 H) .
$$

Pela escolha da seção $s$ e do Lema 2.6.3, temos:

$$
\bar{\omega}_{1 H}(X)=\lambda(X)
$$

para todo $X \in \mathfrak{m}$. De (2.40), temos:

$$
\begin{aligned}
\Gamma_{g H}(v) & =\operatorname{Ad}_{s(g H)}\left(\bar{\omega}_{g H}(v)\right) \\
& =s(g H) \circ \bar{\omega}_{g H}(v) \circ s(g H)^{-1} .
\end{aligned}
$$

Como estamos identificando $T_{1 H}(G / H)$ com $\mathfrak{m}$, temos:

$$
\Gamma_{1 H}(X)=\bar{\omega}_{1 H}(X)
$$

para todo $X \in \mathfrak{m}$. A conclusão segue de (2.68), (2.70), (2.71) e (2.72). 


\section{Capítulo 3}

\section{$G$-estruturas e a Inner Torsion}

\subsection{Introdução}

Neste Capítulo estudaremos a inner torsion de uma $G$-estrutura. A noção de $G$-estrutura é um tanto quanto conhecida nos livros de Geometria Diferencial (ver, por exemplo, [6], [16], [27], [32]). Dar uma G-estrutura em uma variedade diferenciável $M^{n}$ significa escolher um subconjunto dos referenciais do fibrado tangente de $M$ no qual o grupo $G$ age de forma livre e transitiva. Por exemplo, dar uma $\mathrm{O}\left(\mathbb{R}^{n}\right)$-estrutura em $M$ é equivalente a dar uma métrica Riemanniana na variedade $M$.

A inner torsion de uma $G$-estrutura pode ser vista como um tipo de derivada covariante da $G$-estrutura dada. Mais precisamente, dado uma $G$ estrutura $P$, a inner torsion de $P$ é um campo tensorial definido pela derivada covariante de $P$ em relação a uma conexão dada. Por exemplo, consideremos o caso em que $\left(M^{n}, g\right)$ é uma variedade Riemanniana munida de sua conexão de Levi-Civita $\nabla, G$ é o grupo ortogonal $\mathrm{O}\left(\mathbb{R}^{n}\right)$ e a $G$-estrutura é dada pelo conjunto dos referenciais ortonormais. Como $\nabla$ é compatível com $g$, a inner torsion dessa $G$-estrutura é nula.

A inner torsion tem um papel fundamental na teoria das variedades infinitesimalmente homogêneas onde, no Capítulo 4, estudamos um teorema de imersões isométricas em variedades semi-Riemannianas infinitesimalmente homogêneas munidas com uma $G$-estrutura. As principais referências são [26] e [27]. 


\section{$3.2 \quad G$-estruturas em fibrados vetoriais}

Sejam $\left(E, \pi, M, \mathbb{R}^{n}\right)$ um fibrado vetorial, $\operatorname{FR}(E)$ o $\mathrm{GL}\left(\mathbb{R}^{n}\right)$-fibrado principal dos referenciais de $E$ e $G$ um subgrupo de Lie de $\operatorname{GL}\left(\mathbb{R}^{n}\right)$.

Definição 3.2.1 Uma $G$-estrutura no fibrado vetorial $E$ é um $G$-subfibrado principal $P$ de $\operatorname{FR}(E)$.

Exemplo 3.2.2 Sejam $\left(E, \pi, M, \mathbb{R}^{n}\right)$ um fibrado vetorial, $P \subset \mathrm{FR}(E)$ uma $G$-estrutura em $E$ e $f: N \rightarrow M$ uma aplicação diferenciável. Como $f^{*} P$ é um $G$-subfibrado principal de $f^{*} \mathrm{FR}(E)$ (cf. Exemplo 2.1.11), segue que $f^{*} P$ é uma $G$-estrutura de $f^{*} E$.

Exemplo 3.2.3 Sejam $\left(E, \pi, M, \mathbb{R}^{n}\right)$ um fibrado vetorial munido de uma métrica semi-Riemanniana $g$ de índice $r$. Assuma que a fibra típica $\mathbb{R}^{n}$ está munida da forma bilinear de Minkowski $\langle\cdot, \cdot\rangle$ de índice $r$. Para cada $x \in M$, denotemos por $\mathrm{FR}^{\circ}\left(E_{x}\right)$ o conjunto de todas as isometrias lineares de $\mathbb{R}^{n}$ em $E_{x}$. Então, o conjunto

$$
\operatorname{FR}^{\mathrm{o}}(E)=\bigcup_{x \in M} \mathrm{FR}^{\mathrm{o}}\left(E_{x}\right)
$$

de todos os referenciais ortonormais de $E$ é um $\mathrm{O}_{r}\left(\mathbb{R}^{n}\right)$-subfibrado principal de $\operatorname{FR}(E)$. De fato, para cada $x \in M, \mathrm{FR}^{\mathrm{o}}\left(E_{x}\right)$ é uma $\mathrm{O}_{r}\left(\mathbb{R}^{n}\right)$-órbita. Além disso, pela Proposição 2.2.13, existe uma seção local diferenciável $s$ : $U \rightarrow \mathrm{FR}(E)$ de $\mathrm{FR}(E)$, com $x \in U$ e $s(U) \subset \mathrm{FR}^{\circ}(E)$. Isso mostra que $\mathrm{FR}^{\mathrm{o}}(E)$ é um $\mathrm{O}_{r}\left(\mathbb{R}^{n}\right)$-subfibrado principal de $\mathrm{FR}(E)$. Portanto, $\mathrm{FR}^{\mathrm{o}}(E)$ é uma $\mathrm{O}_{r}\left(\mathbb{R}^{n}\right)$-estrutura em $E$.

Exemplo 3.2.4 Seja $(F, \pi, M, V)$ um subfibrado vetorial de $\left(E, \pi, M, \mathbb{R}^{n}\right)$. Então, o conjunto

$$
\operatorname{FR}(E ; V, F)
$$

de todos os referenciais de $E$ adaptados a $(V, F)$ é um $\operatorname{GL}\left(\mathbb{R}^{n} ; V\right)$-subfibrado principal de $\mathrm{FR}(E)$. Assim, $\mathrm{FR}(E ; V, F)$ é uma $\mathrm{GL}\left(\mathbb{R}^{n} ; V\right)$-estrutura no fibrado vetorial $E$. Suponha, além disso, que $E$ está munido de uma métrica semi-Riemanniana $g$ de índice $r$ e $\mathbb{R}^{n}$ está munido da forma bilinear de Minkowski $\langle\cdot, \cdot\rangle$ de índice $r$. Então, o conjunto

$$
\operatorname{FR}^{\mathrm{o}}(E ; V, F)=\mathrm{FR}(E ; V, F) \cap \mathrm{FR}^{\mathrm{o}}(E)
$$

é uma $\mathrm{O}_{r}\left(\mathbb{R}^{n} ; V\right)$-estrutura em $E$ se $\mathrm{FR}^{\mathrm{o}}\left(E_{x} ; \mathbb{R}^{k}, F_{x}\right) \neq \emptyset$, para todo $x \in M$. 
Exemplo 3.2.5 Sejam $\left(E, \pi, M, \mathbb{R}^{n}\right)$ um fibrado vetorial e $\epsilon \in \boldsymbol{\Gamma}(E)$ com $\epsilon(x) \neq 0$, para todo $x \in M$. Se $e_{0} \in \mathbb{R}^{n}$ é um vetor não-nulo, então o conjunto

$$
\operatorname{FR}\left(E ; e_{0}, \epsilon\right)=\bigcup_{x \in M} \operatorname{FR}\left(E_{x} ; e_{0}, \epsilon(x)\right)
$$

de todos os referenciais de $E$ que são adaptados a $\left(e_{0}, \epsilon\right)$ é uma $\operatorname{GL}\left(\mathbb{R}^{n} ; e_{0}\right)$ estrutura em $E$. Se $g$ é uma métrica semi-Riemanniana de índice $r$ em $E$ e $\langle\cdot, \cdot\rangle$ denota a forma bilinear de Minkowski de índice $r$ em $\mathbb{R}^{n}$, então o conjunto

$$
\operatorname{FR}^{\circ}\left(E ; e_{0}, \epsilon\right)=\operatorname{FR}\left(E ; e_{0}, \epsilon\right) \cap \mathrm{FR}^{\circ}(E)
$$

é uma $\mathrm{O}_{r}\left(\mathbb{R}^{n} ; e_{0}\right)$-estrutura em $E$ se $\mathrm{FR}^{\mathrm{o}}\left(E_{x} ; e_{0}, \epsilon(x)\right) \neq 0$, para todo $x \in M$.

Sejam $E, F$ fibrados vetoriais sobre a mesma variedade diferenciável $M$ e com mesma fibra típica $\mathbb{R}^{n}$. Seja $G$ um subgrupo de Lie de $\mathrm{GL}\left(\mathbb{R}^{n}\right)$ e sejam $P, Q G$-estruturas em $E$ e $F$, respectivamente. Dizemos que um morfismo de fibrados vetoriais $L: E \rightarrow F$ preserva $G$-estrutura se:

$$
L_{x} \circ p \in Q_{x}
$$

para quaisquer $x \in M$ e $p \in P_{x}$. A condição (3.1) implica que $L_{x}$ é um isomorfismo linear, para todo $x \in M$. Logo, pelo Lema 2.2.15, todo morfismo de fibrados vetoriais que preserva $G$-estrutura é um isomorfismo de fibrados vetoriais.

Definição 3.2.6 Sejam $M^{n}$ uma variedade diferenciável e $G$ um subgrupo de Lie de $\operatorname{GL}\left(\mathbb{R}^{n}\right)$. Uma $G$-estrutura em $M$ é uma $G$-estrutura $P \subset \operatorname{FR}(T M)$ no fibrado tangente $T M$.

Definição 3.2.7 Sejam $M^{n}, N^{n}$ variedades diferenciáveis, $G$ um subgrupo de Lie de $G L\left(\mathbb{R}^{n}\right)$ e $P, Q G$-estruturas em $M$ e $N$, respectivamente. Dizemos que uma aplicação diferenciável $f: M \rightarrow N$ preserva $G$-estrutura se o morfismo de fibrados vetoriais $\mathrm{d} f: T M \rightarrow f^{*} T N$ preserva $G$-estrutura (cf. Exemplo 2.2.18), onde $f^{*} T N$ está munido com a $G$-estrutura $f^{*} Q$.

Toda aplicação diferenciável $f: M \rightarrow N$ que preserva $G$-estrutura é um difeomorfismo local. Além disso, composição de aplicações que preservam $G$-estrutura é uma aplicação que preserva $G$-estrutura e se $f$ é um difeomorfismo que preserva $G$-estrutura então $f^{-1}$ também é um difeomorfismo que preserva $G$-estrutura. 


\subsection{A inner torsion de uma $G$-estrutura}

Sejam $\left(E, \pi, M, \mathbb{R}^{n}\right)$ um fibrado vetorial, $\nabla$ uma conexão em $E$ e denotemos por $\operatorname{Hor}(\mathrm{FR}(E))$ a conexão em $\mathrm{FR}(E)$ associada a $\nabla$. Consideremos um subgrupo de Lie $G$ de $\operatorname{GL}\left(\mathbb{R}^{n}\right)$ e $P$ uma $G$-estrutura em $E$.

Definição 3.3.1 Dizemos que a conexão $\nabla$ é compatível com a $G$-estrutura $P$ se

$$
\operatorname{Hor}_{p}(\mathrm{FR}(E)) \subset T_{p} P
$$

para todo $p \in P$.

A condição (3.2) significa que a distribuição $\operatorname{Hor}(\mathrm{FR}(E))$ é tangente à subvariedade $P$ de $\operatorname{FR}(E)$. Neste caso, a restrição de $\operatorname{Hor}(\operatorname{FR}(E))$ a $P$ é uma conexão no $G$-fibrado principal $P$. No caso geral, existe um tensor que "mede a falta de compatibilidade"de $\nabla$ com $P$, chamado a inner torsion da $G$-estrutura $P$ em relação a $\nabla$, que passaremos a descrevê-lo.

Para cada $x \in M$, denotemos por $G_{x}$ o subgrupo de $\operatorname{GL}\left(E_{x}\right)$ formado por todas as aplicações $\sigma: E_{x} \rightarrow E_{x}$ que preservam $G$-estrutura, ou seja,

$$
G_{x}=\left\{\sigma \in \mathrm{GL}\left(E_{x}\right): \sigma \circ p \in P_{x} \text {, para algum } p \in P_{x}\right\} .
$$

Lema 3.3.2 Para todo $x \in M, G_{x}$ é um subgrupo de Lie de $\operatorname{GL}\left(E_{x}\right)$.

Demonstração. Mostremos que

$$
\mathcal{I}_{p}(G)=G_{x}
$$

para todo $p \in P_{x}$, onde $\mathcal{I}_{p}: \mathrm{GL}\left(\mathbb{R}^{n}\right) \rightarrow \mathrm{GL}\left(E_{x}\right)$ denota o isomorfismo dado pela conjugação com $p$, i.e., $\mathcal{I}_{p}(q)=p \circ q \circ p^{-1}$, para todo $q \in \operatorname{GL}\left(\mathbb{R}^{n}\right)$. De fato, se $T \in \mathcal{I}_{p}(G)$ então $T=p \circ g \circ p^{-1}$, para algum $g \in G$. Assim, $T \circ p=p \circ g \in P_{x}$ e, portanto, $T \in G_{x}$. Reciprocamente, dado $\sigma \in G_{x}$ temos $\sigma \circ p=p \circ g$, para algum $g \in G$. Assim, $\sigma=p \circ g \circ p^{-1} \in \mathcal{I}_{p}(G)$.

Para cada $x \in M$, denotemos por $\mathfrak{g}_{x} \subset \mathfrak{g} \mathfrak{g}\left(E_{x}\right)$ a álgebra de Lie de $G_{x}$. Assim, de (3.3), temos:

$$
\operatorname{Ad}_{p}(\mathfrak{g})=\mathfrak{g}_{x},
$$

para todo $p \in P_{x}$, onde $\mathfrak{g} \subset \mathfrak{g l}\left(\mathbb{R}^{n}\right)$ denota a álgebra de Lie de $G$. 
Seja $\omega$ a forma de conexão de $\operatorname{Hor}(\operatorname{FR}(E))$. Para cada $x \in M$ e para cada $p \in \operatorname{FR}\left(E_{x}\right)$, a aplicação

$$
\left(\mathrm{d} \Pi_{p}, \omega_{p}\right): T_{p} \mathrm{FR}(E) \rightarrow T_{x} M \oplus \mathfrak{g l}\left(\mathbb{R}^{n}\right),
$$

definida por

$$
\left(\mathrm{d} \Pi_{p}, \omega_{p}\right)(v)=\left(\mathrm{d} \Pi_{p}(v), \omega_{p}(v)\right),
$$

para todo $v \in T_{p} \mathrm{FR}(E)$, é um isomorfismo, onde $\Pi: \mathrm{FR}(E) \rightarrow M$ denota a projeção. De fato, temos uma decomposição em soma direta:

$$
T_{p} \operatorname{FR}(E)=\operatorname{Hor}_{p}(\operatorname{FR}(E)) \oplus \operatorname{Ver}_{p}(\operatorname{FR}(E)) ;
$$

a aplicação $\mathrm{d} \Pi_{p}$ transforma $\operatorname{Hor}_{p}(\operatorname{FR}(E))$ isomorficamente sobre $T_{x} M$ e a aplicação $\omega_{p}$ transforma $\operatorname{Ver}_{p}\left(\operatorname{FR}(E)\right.$ ) isomorficamente sobre $\mathfrak{g l}\left(\mathbb{R}^{n}\right)$ (cf. (2.23)).

Dados $x \in M$ e $p \in P_{x}$, denotemos por

$$
\mathrm{V}_{p}=\left(\mathrm{d} \Pi_{p}, \omega_{p}\right)\left(T_{p} P\right) \subset T_{x} M \oplus \mathfrak{g l}\left(\mathbb{R}^{n}\right)
$$

a imagem de $T_{p} P$ pelo isomorfismo (3.5). Se $\nabla$ é compatível com a $G$ estrutura $P$ então $\mathrm{V}_{p}=T_{x} M \oplus \mathfrak{g}$. Para o caso geral, defineremos um tensor que "mede"o quanto $\mathrm{V}_{p}$ se aproxima de $T_{x} M \oplus \mathfrak{g}$.

Lema 3.3.3 Dados $x \in M$ e $p \in P_{x}$, existe uma única aplicação linear $\mathcal{L}_{p}: T_{x} M \rightarrow \mathfrak{g l}\left(\mathbb{R}^{n}\right) / \mathfrak{g}$ tal que

$$
\mathrm{V}_{p}=\left\{(v, X) \in T_{x} M \oplus \mathfrak{g l}\left(\mathbb{R}^{n}\right): \mathcal{L}_{p}(v)=X+\mathfrak{g}\right\} .
$$

Além disso, se $s: U \rightarrow P$ é uma seção local diferenciável de $P$, $\operatorname{com} s(x)=p$, e $\bar{\omega}=s^{*} \omega$ é a representação de $\omega$ em relação a $s$, então $\mathcal{L}_{p}$ é a composição

$$
T_{x} M \stackrel{\bar{\omega}_{x}}{\longrightarrow} \mathfrak{g l}\left(\mathbb{R}^{n}\right) \stackrel{\text { quociente }}{\longrightarrow} \mathfrak{g l}\left(\mathbb{R}^{n}\right) / \mathfrak{g}
$$

Demonstração. A existência e unicidade da aplicação $\mathcal{L}_{p}$ segue do fato que a restrição a $\mathrm{V}_{p}$ da projeção $T_{x} M \oplus \mathfrak{g l}\left(\mathbb{R}^{n}\right) \rightarrow T_{x} M$ é sobrejetora e $\mathrm{V}_{p} \cap\left(\{0\} \oplus \mathfrak{g l}\left(\mathbb{R}^{n}\right)\right)=\{0\} \oplus \mathfrak{g}$. Seja agora $s: U \rightarrow P$ uma seção local diferenciável de $P$, com $s(x)=p$, e seja $\bar{\omega}=s^{*} \omega$. A imagem de $\mathrm{d} s_{x}$ está contida em $T_{p} P$. Assim, dado $v \in T_{x} M$, temos $\mathrm{d} s_{x}(v) \in T_{p} P$ e a imagem de $\mathrm{d} s_{x}(v)$ pelo isomorfismo (3.5) é igual a

$$
\left(v, \omega_{p}\left(\mathrm{~d} s_{x}(v)\right)\right)=\left(v, \bar{\omega}_{x}(v)\right) .
$$

Assim, o gráfico de $\bar{\omega}$ está contido em $\mathrm{V}_{p}$ e a conclusão segue. 
Observação 3.3.4 A aplicação linear $\bar{\omega}_{x}: T_{x} M \rightarrow \mathfrak{g l}\left(\mathbb{R}^{n}\right)$ depende da escolha da seção local diferenciável $s: U \rightarrow P$, com $s(x)=p$. No entanto, do Lema 3.3.3, a composição (3.7) depende somente da escolha do ponto $p \in P_{x}$.

Sejam $x \in M$ e $p \in P_{x}$. De (3.4) segue que o isomorfismo linear $\operatorname{Ad}_{p}$ : $\mathfrak{g l}\left(\mathbb{R}^{n}\right) \rightarrow \mathfrak{g l}\left(E_{x}\right)$ induz um isomorfismo linear

$$
\overline{\operatorname{Ad}}_{p}: \mathfrak{g l}\left(\mathbb{R}^{n}\right) / \mathfrak{g} \rightarrow \mathfrak{g l}\left(E_{x}\right) / \mathfrak{g}_{x}
$$

por passagem ao quociente.

Lema 3.3.5 A aplicação linear

$$
\mathfrak{I}_{x}^{P}: T_{x} M \rightarrow \mathfrak{g l}\left(E_{x}\right) / \mathfrak{g}_{x}
$$

definida pelo diagrama

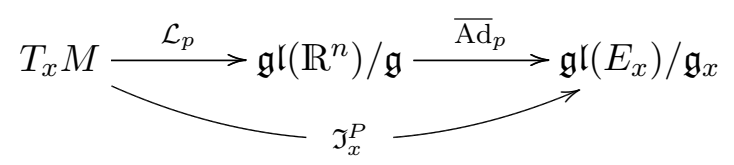

não depende da escolha do ponto $p \in P_{x}$.

Demonstração. Pela Observação 3.3.4, a aplicação linear $\mathcal{L}_{p}$ depende somente em $p$. Consideremos então $p, q \in P_{x}$ e seja $g \in G$ tal que $q=p \cdot g$. Consideremos a seção local diferenciável $s^{\prime}=\gamma_{g} \circ s: U \rightarrow P$ de $P$, onde $\gamma_{g}: P \rightarrow P$ denota a ação de $g$ em $P$. Temos $s^{\prime}(x)=q$. Denotando por $\bar{\omega}^{\prime}=s^{\prime *} \omega$, segue de (2.27) que $\bar{\omega}^{\prime}=\operatorname{Ad}_{g^{-1}} \circ \bar{\omega}$. Temos, assim, o seguinte diagrama comutativo:

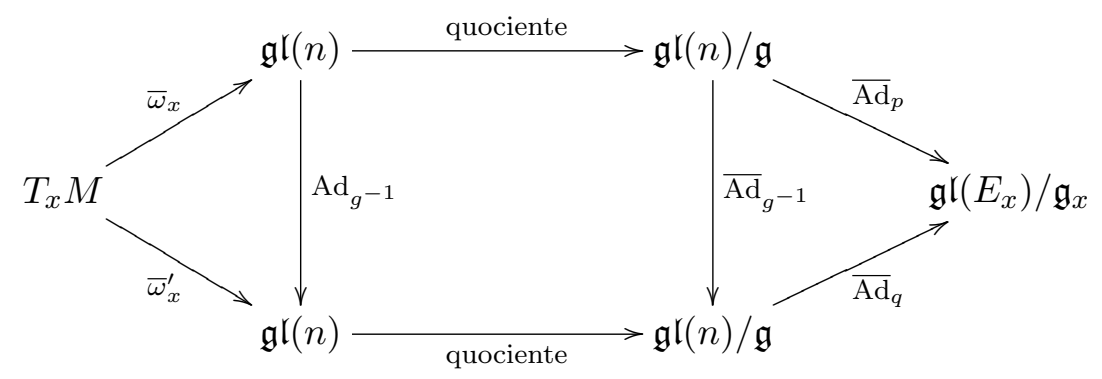

onde $\overline{\operatorname{Ad}}_{g^{-1}}$ é obtida de $\operatorname{Ad}_{g^{-1}}$ por passagem ao quociente, concluindo a prova. 
Definição 3.3.6 A aplicação linear (3.9) é chamada a inner torsion da $G$ estrutura $P$ no ponto $x$ em relação à conexão $\nabla$.

O Lema seguinte proporciona um método mais simples para o cálculo da inner torsion.

Lema 3.3.7 Sejam $s: U \rightarrow P$ uma seção local diferenciável de $P$, com $x \in U$, e $\Gamma$ o tensor de Christoffel da conexão $\nabla$ em relação a $s$. Então, a inner torsion $\mathfrak{I}_{x}^{P}$ é precisamente a composição da aplicação $\Gamma_{x}: T_{x} M \rightarrow \mathfrak{g l}\left(E_{x}\right)$ com a aplicação quociente $\mathfrak{g l}\left(E_{x}\right) \rightarrow \mathfrak{g l}\left(E_{x}\right) / \mathfrak{g}_{x}$

Demonstração. Seja $\omega$ a forma de conexão de $\operatorname{Hor}(\operatorname{FR}(E))$ e seja $\bar{\omega}=s^{*} \omega$. De (2.40) temos $\Gamma_{x}=\operatorname{Ad}_{p} \circ \bar{\omega}_{x}$, onde $p=s(x)$. Temos assim o seguinte diagrama comutativo:

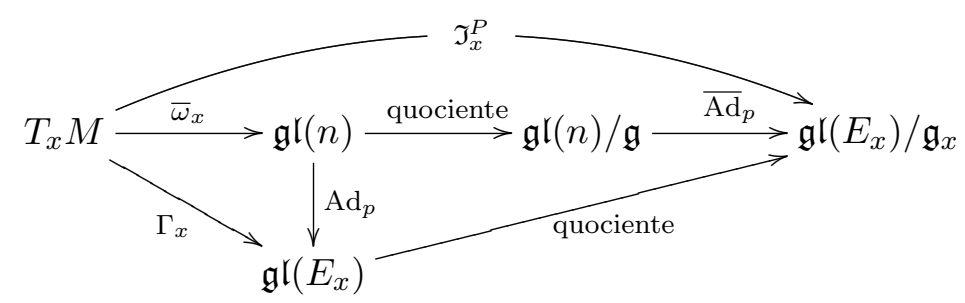

Isso conclui a prova.

Veremos a seguir alguns exemplos do cálculo da inner torsion em situações específicas.

Exemplo 3.3.8 Sejam $\left(E, \pi, M, \mathbb{R}^{n}\right)$ um fibrado vetorial e $s: M \rightarrow \operatorname{FR}(E)$ uma seção global diferenciável de $\operatorname{FR}(E)$. Então, $P=s(M)$ é uma $G$ estrutura em $E$, com $G=\left\{\operatorname{Id}_{\mathbb{R}^{n}}\right\}$. Para cada $x \in M$, temos $G_{x}=\left\{\operatorname{Id}_{E_{x}}\right\}$ e, assim, $\mathfrak{g}_{x}=\{0\}$. Assim, se $\nabla$ é uma conexão em $E$, segue do Lema 3.3.7 que a inner torsion $\mathfrak{I}_{x}^{P}: T_{x} M \rightarrow \mathfrak{g l}\left(E_{x}\right)$ é igual ao tensor de Christoffel $\Gamma_{x}: T_{x} M \rightarrow \mathfrak{g l}\left(E_{x}\right)$ correspondente a $s$.

Exemplo 3.3.9 Seja $\left(E, \pi, M, \mathbb{R}^{n}\right)$ um fibrado vetorial munido de uma métrica semi-Riemanniana $g$ de índice $r$, e seja $\langle\cdot, \cdot\rangle$ a forma bilinear de Minkowski de índice $r$ em $\mathbb{R}^{n}$. Então, $P=\mathrm{FR}^{\mathrm{o}}(E)$ é uma $\mathrm{O}_{r}\left(\mathbb{R}^{n}\right)$-estrutura em $E$, (cf. Exemplo 3.2.3). Dado $x \in M$, temos $G_{x}=\mathrm{O}_{r}\left(E_{x}\right)$, assim $\mathfrak{g}_{x}$ é o espaço dos endomorfismos lineares de $E_{x}$ que são anti-simétricos em relação a $g_{x}$. Podemos identificar $\mathfrak{g l}\left(E_{x}\right) / \mathfrak{g}_{x}$ com o espaço $\operatorname{sym}\left(E_{x}\right)$ dos endomorfismos lineares de $E_{x}$ que são simétricos (em relação a $g_{x}$ ), através do isomorfismo:

$$
T+\mathfrak{g}_{x} \in \mathfrak{g l}\left(E_{x}\right) / \mathfrak{g}_{x} \longmapsto \frac{1}{2}\left(T+T^{*}\right) \in \operatorname{sym}\left(E_{x}\right),
$$


onde $T^{*}: E_{x} \rightarrow E_{x}$ denota o operador transposto de $T$ em relação a $g_{x}$. Sejam $\nabla$ uma conexão em $E$ e $x \in M$. A inner torsion $\mathfrak{I}_{x}^{P}$ pode, então, ser identificada com uma aplicação linear de $T_{x} M$ em $\operatorname{sym}\left(E_{x}\right)$. Sejam $s$ : $U \rightarrow P$ uma seção local diferenciável de $P$, com $x \in U$, e $e, e^{\prime} \in E_{x}$ fixados. Consideremos as seções locais diferenciáveis $\epsilon, \epsilon^{\prime}: U \rightarrow E$ de $E$ definidas por

$$
\begin{aligned}
\epsilon(y) & =\left(s(y) \circ s(x)^{-1}\right) \cdot e \\
\epsilon^{\prime}(y) & =\left(s(y) \circ s(x)^{-1}\right) \cdot e^{\prime},
\end{aligned}
$$

para todo $y \in U$. Como as representações $\widetilde{\epsilon}, \widetilde{\epsilon}$ de $\epsilon$ e $\epsilon^{\prime}$, respectivamente, são constantes, temos:

$$
\begin{aligned}
\nabla_{v} \epsilon & =\Gamma_{x}(v) \cdot \epsilon(x), \\
\nabla_{v} \epsilon^{\prime} & =\Gamma_{x}(v) \cdot \epsilon^{\prime}(x),
\end{aligned}
$$

para todo $v \in T_{x} M$. Como $s(y) \in \mathrm{FR}^{\mathrm{o}}\left(E_{y}\right)$ para todo $y \in U$, temos:

$$
g_{y}\left(\epsilon(y), \epsilon^{\prime}(y)\right)=\left\langle s(x)^{-1} \cdot e, s(x)^{-1} \cdot e^{\prime}\right\rangle,
$$

para todo $y \in U$. Isso implica que a aplicação $g\left(\epsilon, \epsilon^{\prime}\right)$ é constante. Assim:

$$
\begin{aligned}
0 & =v\left(g\left(\epsilon, \epsilon^{\prime}\right)\right)=\nabla_{v} g\left(e, e^{\prime}\right)+g_{x}\left(\nabla_{v} \epsilon, e^{\prime}\right)+g_{x}\left(e, \nabla_{v} \epsilon^{\prime}\right) \\
& =\nabla_{v} g\left(e, e^{\prime}\right)+g_{x}\left(\Gamma_{x}(v) \cdot e, e^{\prime}\right)+g_{x}\left(e, \Gamma_{x}(v) \cdot e^{\prime}\right),
\end{aligned}
$$

para todo $v \in T_{x} M$. Temos:

$$
g_{x}\left(\left(\Gamma_{x}(v)+\Gamma_{x}(v)^{*}\right) \cdot e, e^{\prime}\right)=-\nabla_{v} g\left(e, e^{\prime}\right) .
$$

De (3.11) e do Lema 3.3.7, temos:

$$
g_{x}\left(\mathfrak{I}_{x}^{P}(v), \cdot\right)=\frac{1}{2} g_{x}\left(\left(\Gamma_{x}(v)+\Gamma_{x}(v)^{*}\right), \cdot\right)=-\frac{1}{2} \nabla_{v} g,
$$

para quaisquer $x \in M$ e $v \in T_{x} M$. Usando $g_{x}$ para identificar $\nabla_{v} g: E_{x} \times$ $E_{x} \rightarrow \mathbb{R}$ com um endomorfismo linear de $E_{x}$, obtemos:

$$
\mathfrak{I}_{x}^{P}(v)=-\frac{1}{2} \nabla_{v} g .
$$

Assim, a inner torsion da $G$-estrutura $P$ é a derivada covariante de $g$. Em particular, $\mathfrak{I}^{P}=0$ se, e somente se, $\nabla g=0$. 
Exemplo 3.3.10 Seja $(F, \pi, M, V)$ um subfibrado vetorial de um fibrado vetorial $\left(E, \pi, M, \mathbb{R}^{n}\right)$. Então, $P=\operatorname{FR}(E ; V, F)$ é uma $G=\operatorname{GL}\left(\mathbb{R}^{n} ; V\right)$ estrutura em $E$ (cf. Exemplo 3.2.4). Sejam $\nabla$ uma conexão em $E$ e $x \in M$ um ponto fixado. Temos $G_{x}=\operatorname{GL}\left(E_{x} ; F_{x}\right)$ e $\mathfrak{g}_{x}$ é o espaço vetorial dos endomorfismos lineares $T: E_{x} \rightarrow E_{x}$ que satisfazem $T\left(F_{x}\right) \subset F_{x}$. O quociente $\mathfrak{g l}\left(E_{x}\right) / \mathfrak{g}_{x}$ pode ser identificado com o espaço $\operatorname{Lin}\left(F_{x}, E_{x} / F_{x}\right)$ através do isomorfismo:

$$
T+\mathfrak{g}_{x} \in \mathfrak{g r}\left(E_{x}\right) /\left.\mathfrak{g}_{x} \longmapsto \mathfrak{q} \circ T\right|_{F_{x}} \in \operatorname{Lin}\left(F_{x}, E_{x} / F_{x}\right),
$$

onde $\mathfrak{q}: E_{x} \rightarrow E_{x} / F_{x}$ denota a aplicação quociente. Assim, a inner torsion $\mathfrak{I}_{x}^{P}$ está identificada com uma aplicação linear de $T_{x} M$ em $\operatorname{Lin}\left(F_{x}, E_{x} / F_{x}\right)$. Seja $s: U \rightarrow P$ uma seção local diferenciável, com $x \in U$. Dado $e \in F_{x}$, defina uma seção local $\epsilon: U \rightarrow E$ de $E$ por

$$
\epsilon(y)=s(y) \cdot s(x)^{-1} \cdot e,
$$

para todo $y \in U$. Como a representação $\widetilde{\epsilon}$ de $\epsilon$ em relação a $s$ é constante, obtemos:

$$
\nabla_{v} \epsilon=\Gamma_{x}(v) \cdot \epsilon(x)
$$

para todo $v \in T_{x} M$. Como $s$ toma valores em $\operatorname{FR}(E ; V, F)$, temos $\epsilon(U) \subset F$ e, assim,

$$
\nabla_{v} \epsilon+F_{x}=\alpha_{x}^{F}(v, e) \in E_{x} / F_{x}
$$

onde $\alpha^{F}$ denota a segunda forma fundamental de $F$ (cf. Exemplo 2.4.13). Então:

$$
\Gamma_{x}(v) \cdot e+F_{x}=\alpha_{x}^{F}(v, e)
$$

e

$$
\mathfrak{I}_{x}^{P}(v)=\alpha_{x}^{F}(v, \cdot) \in \operatorname{Lin}\left(F_{x}, E_{x} / F_{x}\right),
$$

para quaisquer $x \in M$ e $v \in T_{x} M$. Em particular, $\mathfrak{I}^{P}=0$ se, e somente se, $\alpha^{F}=0$, i.e., a derivada covariante de qualquer seção diferenciável de $F$ é uma seção diferenciável de $F$.

Exemplo 3.3.11 Seja $(F, \pi, M, V)$ um subfibrado vetorial de um fibrado vetorial $\left(E, \pi, M, \mathbb{R}^{n}\right), g$ uma métrica semi-Riemanniana de índice $r$ em $E$ e $\langle\cdot, \cdot\rangle$ a forma bilinear de Minkowski de índice $r$ em $\mathbb{R}^{n}$. Assumimos que $\mathrm{FR}^{\mathrm{o}}\left(E_{x} ; V, F\right) \neq \emptyset$, para todo $x \in M$, de modo que $P=\mathrm{FR}^{\mathrm{o}}(E ; V, F)$ é uma $\mathrm{O}_{r}\left(\mathbb{R}^{n} ; V\right.$ )-estrutura em $E$ (cf. Exemplo 3.2.4) e que a restrição de $g_{x}$ a $F_{x} \times F_{x}$ é não-degenerada, para todo $x \in M$; assim, $E=F \oplus F^{\perp}$. Denotemos por $\mathfrak{q}: E \rightarrow F^{\perp}$ a projeção. Sejam $\nabla$ uma conexão em $E$ e 
$x \in M$ um ponto fixado. Temos $G_{x}=\mathrm{O}_{r}\left(E_{x} ; F_{x}\right)$ e $\mathfrak{g}_{x}$ é a álgebra de Lie dos endomorfismos lineares $T: E_{x} \rightarrow E_{x}$ que são anti-simétricos (em relação a $\left.g_{x}\right)$ e satisfazem $T\left(F_{x}\right) \subset F_{x}$. O quociente $\mathfrak{g l}\left(E_{x}\right) / \mathfrak{g}_{x}$ pode ser identificado com o espaço $\operatorname{sym}\left(E_{x}\right) \oplus \operatorname{Lin}\left(F_{x}, F_{x}^{\perp}\right)$ através do isomorfismo:

$$
\begin{aligned}
\mathfrak{g l}\left(E_{x}\right) / \mathfrak{g}_{x} & \longrightarrow \operatorname{sym}\left(E_{x}\right) \oplus \operatorname{Lin}\left(F_{x}, F_{x}^{\perp}\right) \\
T+\mathfrak{g}_{x} & \longmapsto\left(\frac{1}{2}\left(T+T^{*}\right),\left.\frac{1}{2} \mathfrak{q}_{x} \circ\left(T-T^{*}\right)\right|_{F_{x}}\right) .
\end{aligned}
$$

Assim, a inner torsion $\mathfrak{I}_{x}^{P}$ está identificada com uma aplicação linear de $T_{x} M$ $\operatorname{em} \operatorname{sym}\left(E_{x}\right) \oplus \operatorname{Lin}\left(F_{x}, F_{x}^{\perp}\right)$. Consideremos a componente

$$
\alpha \in \boldsymbol{\Gamma}\left(\operatorname{Lin}\left(T M, F ; F^{\perp}\right)\right)
$$

de $\nabla$ em relação à decomposição $E=F \oplus F^{\perp}$. Seja $s: U \rightarrow P$ uma seção local diferenciável, com $x \in U$. Como no Exemplo 3.3.9, temos:

$$
\frac{1}{2}\left(\Gamma_{x}(v)+\Gamma_{x}(v)^{*}\right)=-\frac{1}{2} \nabla_{v} g
$$

para todo $v \in T_{x} M$. Além disso, seguindo o raciocínio do Exemplo 3.3.10, obtemos:

$$
\mathfrak{q}\left(\Gamma_{x}(v) \cdot e\right)=\alpha_{x}^{F}(v, e),
$$

para quaisquer $v \in T_{x} M$ e $e \in E_{x}$. Então:

$$
\begin{aligned}
\frac{1}{2}\left(\Gamma_{x}(v)-\Gamma_{x}(v)^{*}\right) & =\Gamma_{x}(v)-\frac{1}{2}\left(\Gamma_{x}(v)+\Gamma_{x}(v)^{*}\right) \\
& =\Gamma_{x}(v)+\frac{1}{2} \nabla_{v} g
\end{aligned}
$$

e

$$
\mathfrak{I}_{x}^{P}(v)=\left(-\frac{1}{2} \nabla_{v} g, \alpha_{x}^{F}(v, \cdot)+\left.\frac{1}{2} \mathfrak{q} \circ \nabla_{v} g\right|_{F_{x}}\right),
$$

para quaisquer $x \in M$ e $v \in T_{x} M$, onde $\nabla_{v} g$ está identificado com um endomorfismo linear de $E_{x}$. Em particular, $\mathfrak{I}^{P}=0$ se, e somente se, $\nabla g=0$ e $\alpha=0$. i.e., se, e somente se, $\nabla$ é compatível com $g$ e a derivada covariante de toda seção diferenciável de $F$ é uma seção diferenciável de $F$.

Exemplo 3.3.12 Sejam $\left(E, \pi, M, \mathbb{R}^{n}\right)$ um fibrado vetorial, $\epsilon \in \boldsymbol{\Gamma}(E)$ tal que $\epsilon(x) \neq 0$, para todo $x \in M$, e $e_{0} \in \mathbb{R}^{n}$ um vetor não-nulo. Então, $P=$ $\operatorname{FR}\left(E ; e_{0}, \epsilon\right)$ é uma $\operatorname{GL}\left(\mathbb{R}^{n} ; e_{0}\right)$-estrutura em $E$ (cf. Exemplo 3.2.5). Seja $\nabla$ uma conexão em $E$. Dado $x \in M$, temos $G_{x}=\mathrm{GL}\left(E_{x} ; \epsilon(x)\right)$ e $\mathfrak{g}_{x}$ é o espaço 
vetorial dos endomorfismos lineares $T: E_{x} \rightarrow E_{x}$ que satisfazem $T(\epsilon(x))=0$. Identifiquemos o quociente $\mathfrak{g l}\left(E_{x}\right) / \mathfrak{g}_{x} \operatorname{com} E_{x}$ através do isomorfismo linear:

$$
T+\mathfrak{g}_{x} \in \mathfrak{g l}\left(E_{x}\right) / g_{x} \longmapsto T(\epsilon(x)) \in E_{x} .
$$

Assim, a inner torsion está identificada com uma aplicação linear de $T_{x} M$ em $E_{x}$. Seja $s: U \rightarrow \operatorname{FR}\left(E ; e_{0}, \epsilon\right)$ uma seção local diferenciável, com $x \in U$. Como a representação $\widetilde{\epsilon}$ de $\epsilon$ satisfaz

$$
\widetilde{\epsilon}(y)=s(y)^{-1} \cdot \epsilon(y)=e_{0},
$$

para todo $y \in U$, segue que

$$
\nabla_{v} \epsilon=\Gamma_{x}(v) \cdot \epsilon(x)
$$

para todo $v \in T_{x} M$. Assim, temos que $\mathfrak{I}_{x}^{P}(v)=\nabla_{v} \epsilon$, para todo $v \in T_{x} M$ e, portanto,

$$
\mathfrak{I}_{x}^{P}=\nabla \epsilon(x),
$$

para todo $x \in M$. Em particular, $\mathfrak{I}^{P}=0$ se, e somente se, $\nabla \epsilon=0$.

Exemplo 3.3.13 Sejam $\left(E, \pi, M, \mathbb{R}^{n}\right)$ um fibrado vetorial, $\epsilon \in \boldsymbol{\Gamma}(E)$ com $\epsilon(x) \neq 0$, para todo $x \in M$, e $e_{0} \in \mathbb{R}^{n}$ um vetor não-nulo. Sejam $g$ uma métrica semi-Riemanniana de índice $r$ em $E$, e $\langle\cdot, \cdot\rangle$ a forma bilinear de Minkowski de índice $r$ em $\mathbb{R}^{n}$ tal que $\mathrm{FR}^{\circ}\left(E_{x} ; e_{0}, \epsilon(x)\right) \neq \emptyset$, para todo $x \in M$. Então, $P=\mathrm{FR}^{\circ}\left(E ; e_{0}, \epsilon\right)$ é uma $\mathrm{O}_{r}\left(\mathbb{R}^{n} ; e_{0}\right)$-estrutura em $E$ (cf. Exemplo 3.2.5). Dado $x \in M$, temos $G_{x}=\mathrm{O}_{r}\left(E_{x} ; \epsilon(x)\right)$ e $\mathfrak{g}_{x}$ é a álgebra de Lie dos endomorfismos lineares anti-simétricos $T: E_{x} \rightarrow E_{x}$ tais que $T(\epsilon(x))=0$. Temos o seguinte isomorfismo linear:

$T+\mathfrak{g}_{x} \in \mathfrak{g l}\left(E_{x}\right) / \mathfrak{g}_{x} \longmapsto\left(\frac{1}{2}\left(T+T^{*}\right), \frac{1}{2}\left(T-T^{*}\right) \cdot \epsilon(x)\right) \in \operatorname{sym}\left(E_{x}\right) \oplus \epsilon(x)^{\perp}$,

onde $\epsilon(x)^{\perp}$ denota o núcleo de $g_{x}(\epsilon(x), \cdot)$. Seja $s: U \rightarrow P$ uma seção local diferenciável, com $x \in U$. Como no Exemplo 3.3.9, temos:

$$
\frac{1}{2}\left(\Gamma_{x}(v)+\Gamma_{x}(v)^{*}\right)=-\frac{1}{2} \nabla_{v} g,
$$

e, como em (3.12), temos:

$$
\frac{1}{2}\left(\Gamma_{x}(v)-\Gamma_{x}(v)^{*}\right)=\Gamma_{x}(v)+\frac{1}{2} \nabla_{v} g,
$$


para todo $x \in M$. Além disso, a igualdade (3.13) está satisfeita. Então,

$$
\frac{1}{2}\left(\Gamma_{x}(v)-\Gamma_{x}(v)^{*}\right) \cdot \epsilon(x)=\nabla_{v} \epsilon+\frac{1}{2}\left(\nabla_{v} g\right)(\epsilon(x)) .
$$

Assim,

$$
\mathfrak{I}_{x}^{P}(v)=\left(-\frac{1}{2} \nabla_{v} g, \nabla_{v} \epsilon+\frac{1}{2}\left(\nabla_{v} g\right)(\epsilon(x))\right),
$$

para quaisquer $x \in M$ e $v \in T_{x} M$. Em particular, $\mathfrak{I}^{P}=0$ se, e somente se, $\nabla g=0$ e $\nabla \epsilon=0$.

\subsection{A inner torsion de $G$-estruturas em espaços ho- mogêneos}

A fim de evitar confusão de notação, um espaço homogêneo será denotado por $K / H$, onde $K$ é um grupo de Lie e $H$ é um subgrupo fechado de $K$. Denotemos por $\mathfrak{k}$ e $\mathfrak{h}$ as álgebras de Lie de $K$ e $H$, respectivamente, e fixemos um subespaço $\mathfrak{m}$ de $\mathfrak{k}, \operatorname{com} \mathfrak{k}=\mathfrak{h} \oplus \mathfrak{m}$.

Fixemos um isomorfismo linear $\mathfrak{i}: \mathbb{R}^{n} \rightarrow \mathfrak{m}$ e consideremos o isomorfismo de grupos de Lie

$$
\mathcal{I}_{\mathfrak{i}}: \operatorname{GL}\left(\mathbb{R}^{n}\right) \rightarrow \mathrm{GL}(\mathfrak{m})
$$

definido por $\mathcal{I}_{\mathfrak{i}}(\tau)=\mathfrak{i} \circ \tau \circ \mathfrak{i}^{-1}$, para todo $\tau \in \mathrm{GL}\left(\mathbb{R}^{n}\right)$. Consideremos o grupo de Lie

$$
G^{\mathfrak{i}}=\mathcal{I}_{\mathfrak{i}}^{-1}(\overline{\operatorname{Ad}}(H)) \subset \mathrm{GL}\left(\mathbb{R}^{n}\right),
$$

onde $\overline{\mathrm{Ad}}: H \rightarrow \mathrm{GL}(\mathfrak{m})$ denota a representação isotrópica de $H$ em $\mathfrak{m}$, definida em (1.53). Consideremos a ação diferenciável à esquerda de $K$ em $\operatorname{FR}(T(K / H))$ definida em $(2.45)$ e seja

$$
P^{\mathfrak{i}} \subset \operatorname{FR}(T(K / H))
$$

a $K$-órbita de $\mathfrak{i}: \mathbb{R}^{n} \rightarrow \mathfrak{m}$, i.e.,

$$
P^{\mathfrak{i}}=\left\{\mathrm{d} \bar{L}_{g}(1 H) \circ \mathfrak{i}: g \in K\right\} .
$$

O conjunto $P^{\mathfrak{i}}$ é uma $G^{\mathfrak{i}}$-estrutura em $K / H$; mais precisamente, dado $g H \in$ $K / H$, a órbita $P_{g H}^{\mathrm{i}}$ é o conjunto

$$
P_{g H}^{\mathfrak{i}}=\left\{\mathrm{d} \bar{L}_{g}(1 H) \circ \mathrm{d} \bar{L}_{h}(1 H) \circ \mathfrak{i}: h \in H\right\} .
$$


O grupo $G_{1 H}^{\mathrm{i}}$ de todos os endomorfismos de $T_{1 H}(K / H)=\mathfrak{m}$ que preservam a $G^{\mathrm{i}}$-estrutura é igual a $\overline{\operatorname{Ad}}(H)$, ou seja,

$$
G_{1 H}^{\mathrm{i}}=\left\{\sigma \in \mathrm{GL}(\mathfrak{m}): \sigma \circ p \in P_{1 H}^{\mathrm{i}}, p \in P_{1 H}^{\mathfrak{i}}\right\}=\overline{\operatorname{Ad}}(H)
$$

(cf. (1.56)). Sua álgebra de Lie $\mathfrak{g}_{1 H}^{\mathfrak{i}}$ é, então, igual a $\overline{\operatorname{ad}}(\mathfrak{h})$.

Para determinar a inner torsion

$$
\mathfrak{I}_{1 H}^{P^{\mathfrak{i}}}: \mathfrak{m} \rightarrow \mathfrak{g l}(\mathfrak{m}) / \overline{\operatorname{ad}}(\mathfrak{h})
$$

da $G^{\mathfrak{i}}$-estrutura $P^{\mathfrak{i}}$ em $K / H$, basta calcular a inner torsion da $G$-estrutura:

$$
P=\gamma_{\mathfrak{i}}^{-1}\left(P^{\mathfrak{i}}\right)=\left\{\mathrm{d} \bar{L}_{g}: g \in K\right\} \subset \mathrm{FR}_{\mathfrak{m}}(T(K / H)),
$$

onde $G=\mathcal{I}_{\mathfrak{i}}\left(G^{\mathfrak{i}}\right)=\overline{\operatorname{Ad}}(H)$ e $\gamma_{\mathfrak{i}}$ é o isomorfismo de fibrados principais definido em (2.44). Note que $P$ é justamente a imagem do morfismo de fibrados principais $\phi$ definido em (2.56).

Proposição 3.4.1 Seja $\nabla$ uma conexão $K$-invariante em $K / H$ correspondente a uma aplicação linear $\lambda: \mathfrak{m} \rightarrow \mathfrak{g l}(\mathfrak{m})$, como no Lema 2.6.2. A inner torsion (3.16) da $\overline{\operatorname{Ad}}(H)$-estrutura (3.17) em $K / H$ é igual a composição de $\lambda: \mathfrak{m} \rightarrow \mathfrak{g l}(\mathfrak{m})$ com a aplicação quociente $\mathfrak{q}: \mathfrak{g l}(\mathfrak{m}) \rightarrow \mathfrak{g l}(\mathfrak{m}) / \overline{\operatorname{ad}}(\mathfrak{h})$.

Demonstração. Seja $s: U \subset K / H \rightarrow K$ uma seção local diferenciável da aplicação quociente $\pi: K \rightarrow K / H$, com $1 H \in U, s(1 H)=1$ e tal que $\mathrm{d} s(1 H): \mathfrak{m} \rightarrow \mathfrak{k}$ seja a aplicação inclusão de $\mathfrak{m}$ em $\mathfrak{k}$ (cf. Observação 2.6.6). Note que $\phi \circ s$ é uma seção local diferenciável de $P \rightarrow K / H$, onde $\phi$ é dado em (2.56). Seja $\bar{\omega}=(\phi \circ s)^{*} \omega$, onde $\omega$ denota a forma de conexão de $\nabla$ em $\operatorname{FR}_{\mathfrak{m}}(T(K / H))$. Pelo diagrama (3.10), a fim de concluir a prova, é suficiente mostrar que $\bar{\omega}_{1 H}$ é igual a $\lambda$. Temos $\bar{\omega}=s^{*}\left(\phi^{*} \omega\right)$ e, portanto,:

$$
\bar{\omega}_{1 H}=\left(\phi^{*} \omega\right)_{1} \circ \mathrm{d} s(1 H) .
$$

A conclusão segue então diretamente de (2.62). 


\section{Capítulo 4}

\section{Imersões Isométricas}

\subsection{Introdução}

O objetivo principal deste Capítulo é estudarmos um teorema de imersões isométricas em variedades semi-Riemannianas infinitesimalmente homogêneas munidas com uma $G$-estrutura (cf. Teorema 4.5.2). Infinitesimalmente homogênea significa que a curvatura e a torção da conexão, bem como a inner torsion da $G$-estrutura, podem ser descritos unicamente em termos da $G$-estrutura, ou seja, são constantes nos referenciais que pertencem a $G$-estrutura.

Por exemplo, consideremos o caso em que $\left(M^{n}, g\right)$ é uma variedade Riemanniana munida de sua conexão de Levi-Civita $\nabla, G$ é o grupo ortogonal $\mathrm{O}\left(\mathbb{R}^{n}\right)$ e a $G$-estrutura é dada pelo conjunto dos referenciais ortonormais. Como $\nabla$ é compatível com $g$, o tensor de torção e a inner torsion dessa $G$-estrutura são nulos. A condição para que o tensor de curvatura seja constante nos referenciais ortonormais é equivalente à condição de que $M$ tenha curvatura seccional constante. Neste caso, o Teorema 4.5.2 reproduz o clássico Teorema Fundamental de Imersões Isométricas em espaços de curvatura seccional constante.

O Teorema 4.5.2, o qual está provado em [26], vale em um contexto mais geral. Mais precisamente, em [26], os autores provam um teorema de imersões afins que preservam $G$-estrutura entre variedades afins. A prova (da versão local) do Teorema consiste, na sua essência, de uma aplicação do Teorema de Frobenius na linguagem de formas diferenciais (ver [26, Teorema 7.4]). Como nosso interesse é o caso semi-Riemanniano, o Teorema 4.5.2, apresentado na Seção 4.5, é a versão do Teorema 7.4 de [26] para o caso 
semi-Riemanniano.

\subsection{As componentes de uma conexão}

Sejam $\left(E, \pi, M, \mathbb{R}^{n}\right)$ um fibrado vetorial e $E^{1}, E^{2}$ subfibrados vetoriais de $E$ tais que $E=E^{1} \oplus E^{2}$. Denotemos por $\operatorname{pr}_{i}: E \rightarrow E^{i}, i=1,2$, as projeções correspondentes. Dado uma conexão $\nabla$ em $E$, definimos:

$$
\begin{aligned}
\nabla_{X}^{1} \epsilon_{1} & =\operatorname{pr}_{1}\left(\nabla_{X} \epsilon_{1}\right) \in \boldsymbol{\Gamma}\left(E^{1}\right), \\
\nabla_{X}^{2} \epsilon_{2} & =\operatorname{pr}_{2}\left(\nabla_{X} \epsilon_{2}\right) \in \boldsymbol{\Gamma}\left(E^{2}\right), \\
\alpha^{1}\left(X, \epsilon_{2}\right) & =\operatorname{pr}_{1}\left(\nabla_{X} \epsilon_{2}\right) \in \boldsymbol{\Gamma}\left(E^{1}\right), \\
\alpha^{2}\left(X, \epsilon_{1}\right) & =\operatorname{pr}_{2}\left(\nabla_{X} \epsilon_{1}\right) \in \boldsymbol{\Gamma}\left(E^{2}\right),
\end{aligned}
$$

para quaisquer $X \in \boldsymbol{\Gamma}(T M), \epsilon_{1} \in \boldsymbol{\Gamma}\left(E^{1}\right)$ e $\epsilon_{2} \in \boldsymbol{\Gamma}\left(E^{2}\right)$. Temos que $\nabla^{1}$, $\nabla^{2}$ são conexões em $E^{1}$ e $E^{2}$, respectivamente. Além disso, $\alpha^{1}$ e $\alpha^{2}$ são aplicações $\mathrm{C}^{\infty}(M)$-bilineares e, portanto, podem ser identificadas com seções diferenciáveis

$$
\begin{gathered}
\alpha^{1} \in \boldsymbol{\Gamma}\left(\operatorname{Lin}\left(T M, E^{2} ; E^{1}\right)\right), \\
\alpha^{2} \in \boldsymbol{\Gamma}\left(\operatorname{Lin}\left(T M, E^{1} ; E^{2}\right)\right) .
\end{gathered}
$$

Definição 4.2.1 As aplicações $\nabla^{1}, \nabla^{2}, \alpha^{1}$ e $\alpha^{2}$, definidas em (4.1), são chamadas as componentes da conexão $\nabla$ em relação à decomposição $E=$ $E^{1} \oplus E^{2}$.

Reciprocamente, dados uma conexão $\nabla^{1}$ em $E^{1}$, uma conexão $\nabla^{2}$ em $E^{2}$ e seções diferenciáveis $\alpha^{1} \in \boldsymbol{\Gamma}\left(\operatorname{Lin}\left(T M, E^{2} ; E^{1}\right)\right), \alpha^{2} \in \boldsymbol{\Gamma}\left(\operatorname{Lin}\left(T M, E^{1} ; E^{2}\right)\right)$, existe uma única conexão $\nabla$ em $E$ cujas componentes são $\nabla^{1}, \nabla^{2}, \alpha^{1}$ e $\alpha^{2}$. Mais precisamente, definimos $\nabla$ por:

$$
\nabla_{X} \epsilon=\nabla_{X}^{1}\left(\operatorname{pr}_{1} \circ \epsilon\right)+\alpha^{1}\left(X, \operatorname{pr}_{2} \circ \epsilon\right)+\nabla_{X}^{2}\left(\operatorname{pr}_{2} \circ \epsilon\right)+\alpha^{2}\left(X, \operatorname{pr}_{1} \circ \epsilon\right),
$$

para quaisquer $X \in \boldsymbol{\Gamma}(T M)$ e $\epsilon \in \boldsymbol{\Gamma}(E)$.

Exemplo 4.2.2 Seja $f: M \rightarrow \bar{M}$ uma imersão isométrica. Então, as componentes da conexão de Levi-Civita $\bar{\nabla}$ de $\bar{M}$, relativo à decomposição $\left.T \bar{M}\right|_{f(M)}=T M \oplus T M^{\perp}$, são a conexão de Levi-Civita $\nabla$ de $M$, a conexão normal $\nabla^{\perp}$ de $f$, a segunda forma fundamental $\alpha$ de $f$ e a forma de Weingarten $A$ da imersão $f$, a qual é dada por

$$
\langle\alpha(X, Y), \eta\rangle=-\langle A(X, \eta), Y\rangle,
$$

para quaisquer $X, Y \in \boldsymbol{\Gamma}(T M)$ e $\eta \in \boldsymbol{\Gamma}\left(T M^{\perp}\right)$. 
Observação 4.2.3 Sejam $g^{1}, g^{2}$ métricas semi-Riemannianas em $E^{1}$ e $E^{2}$, respectivamente, e $g$ a métrica semi-Riemanniana em $E$ dada pela soma direta ortogonal de $g^{1}$ e $g^{2}$. Então, uma conexão $\nabla$ em $E$ com componentes $\nabla^{1}, \nabla^{2}, \alpha^{1}$ e $\alpha^{2}$ é compatível com $g$ (i.e., $\nabla g=0$ ) se, e somente se, $\nabla^{i}$ é compatível com $g^{i}, i=1,2$, e vale a seguinte relação entre $\alpha^{1}$ e $\alpha^{2}$ :

$$
g^{2}\left(\alpha^{2}\left(X, \epsilon_{1}\right), \epsilon_{2}\right)+g^{1}\left(\epsilon_{1}, \alpha^{1}\left(X, \epsilon_{2}\right)\right)=0,
$$

para quaisquer $X \in \boldsymbol{\Gamma}(T M), \epsilon_{1} \in \boldsymbol{\Gamma}\left(E^{1}\right)$ e $\epsilon_{2} \in \boldsymbol{\Gamma}\left(E^{2}\right)$. Essa equivalência segue de (4.1), (4.2) e do fato de que

$$
\nabla g=0 \quad \Longleftrightarrow \quad X\left(g\left(\epsilon_{1}, \epsilon_{2}\right)\right)=g\left(\nabla_{X} \epsilon_{1}, \epsilon_{2}\right)+g\left(\epsilon_{1}, \nabla_{X} \epsilon_{2}\right),
$$

para quaisquer $X \in \boldsymbol{\Gamma}(T M)$ e $\epsilon_{1}, \epsilon_{2} \in \boldsymbol{\Gamma}(E)$. Assim, no contexto de conexões compatíveis com métricas semi-Riemannianas, diremos que $\nabla^{1}, \nabla^{2}$ e $\alpha^{2}$ são as componentes da conexão $\nabla$ em relação à decomposição $E=E^{1} \oplus E^{2}$, entendendo implicitamente que $\alpha^{1}$ é determinada pela relação (4.3).

Consideremos $g^{1}, g^{2}$ métricas semi-Riemannianas em $E^{1}$ e $E^{2}$, respectivamente, com conexões compatíveis $\nabla^{1}$ e $\nabla^{2}$. Denotemos por $g$ a métrica Riemanniana em $E$ dada pela soma direta ortogonal de $g^{1}$ e $g^{2}$, e seja $\nabla$ a conexão em $E$ cujas componentes são $\nabla^{1}, \nabla^{2}, \alpha^{2}$.

Lema 4.2.4 (Equações generalizadas) Se $R, R^{1}, R^{2}$ denotam os tensores de curvatura de $\nabla, \nabla^{1}$ e $\nabla^{2}$, respectivamente, temos:

$$
\begin{aligned}
g\left(R(X, Y) \epsilon_{1}, \eta_{1}\right)= & g^{1}\left(R^{1}(X, Y) \epsilon_{1}, \eta_{1}\right)+g^{2}\left(\alpha^{2}\left(X, \epsilon_{1}\right), \alpha^{2}\left(Y, \eta_{1}\right)\right) \\
& -g^{2}\left(\alpha^{2}\left(X, \eta_{1}\right), \alpha^{2}\left(Y, \epsilon_{1}\right)\right) \\
g\left(R(X, Y) \epsilon_{2}, \eta_{2}\right)= & g^{2}\left(R^{2}(X, Y) \epsilon_{2}, \eta_{2}\right)+g^{1}\left(\alpha^{1}\left(X, \epsilon_{2}\right), \alpha^{1}\left(Y, \eta_{2}\right)\right) \\
& -g^{1}\left(\alpha^{1}\left(X, \eta_{2}\right), \alpha^{1}\left(Y, \epsilon_{2}\right)\right)
\end{aligned}
$$

para quaisquer $X, Y \in \boldsymbol{\Gamma}(T M), \epsilon_{1}, \eta_{1} \in \boldsymbol{\Gamma}\left(E^{1}\right)$ e $\epsilon_{2}, \eta_{2} \in \boldsymbol{\Gamma}\left(E^{2}\right)$. Além disso, dado uma conexão $\nabla^{M}$ em $M$ com torção $T$, temos:

$$
\begin{aligned}
g\left(R(X, Y) \epsilon_{1}, \eta_{2}\right)= & g^{2}\left(\left(\nabla_{X}^{2} \alpha^{2}\right)\left(Y, \epsilon_{1}\right), \eta_{2}\right)-g^{2}\left(\left(\nabla_{Y}^{2} \alpha^{2}\right)\left(X, \epsilon_{1}\right), \eta_{2}\right) \\
& +g^{2}\left(\alpha^{2}\left(T(X, Y), \epsilon_{1}\right), \eta_{2}\right), \\
g\left(R(X, Y) \epsilon_{2}, \eta_{1}\right)= & g^{1}\left(\left(\nabla_{X}^{1} \alpha^{1}\right)\left(Y, \epsilon_{2}\right), \eta_{1}\right)-g^{1}\left(\left(\nabla_{Y}^{1} \alpha^{1}\right)\left(X, \epsilon_{2}\right), \eta_{1}\right) \\
& +g^{1}\left(\alpha^{1}\left(T(X, Y), \epsilon_{2}\right), \eta_{1}\right) .
\end{aligned}
$$


Se $\iota=\left(\iota_{1}, \iota_{2}\right): T M \rightarrow E$ é um morfismo de fibrados vetoriais, então a $\iota$-torção de $\nabla$ satisfaz:

$$
\begin{aligned}
g\left(T^{\iota}(X, Y), \epsilon_{1}\right)= & g^{1}\left(T^{\iota_{1}}(X, Y), \epsilon_{1}\right)+g^{1}\left(\alpha^{1}\left(X, \iota_{2}(Y)\right), \epsilon_{1}\right) \\
& -g^{1}\left(\alpha^{1}\left(Y, \iota_{2}(X)\right), \epsilon_{1}\right), \\
g\left(T^{\iota}(X, Y), \epsilon_{2}\right)= & g^{2}\left(T^{\iota 2}(X, Y), \epsilon_{2}\right)+g^{2}\left(\alpha^{2}\left(X, \iota_{1}(Y)\right), \epsilon_{2}\right) \\
& -g^{2}\left(\alpha^{2}\left(Y, \iota_{1}(X)\right), \epsilon_{2}\right),
\end{aligned}
$$

onde $T^{\iota_{1}}, T^{\iota_{2}}$ denotam a $\iota_{1}$-torção de $\nabla^{1}$ e a $\iota_{2}$-torção de $\nabla^{2}$, respectivamente.

Demonstração. Usando (4.2), temos:

$$
\begin{aligned}
g\left(R(X, Y) \epsilon_{1}, \eta_{1}\right)= & g\left(\nabla_{X} \nabla_{Y} \epsilon_{1}-\nabla_{Y} \nabla_{X} \epsilon_{1}-\nabla_{[X, Y]} \epsilon_{1}, \eta_{1}\right) \\
= & g\left(\nabla_{X}^{1} \nabla_{Y}^{1} \epsilon_{1}+\alpha^{2}\left(X, \nabla_{Y}^{1} \epsilon_{1}\right)+\alpha^{1}\left(X, \alpha^{2}\left(Y, \epsilon_{1}\right)\right)\right. \\
& +\nabla_{X}^{2} \alpha^{2}\left(Y, \epsilon_{1}\right)-\nabla_{Y}^{1} \nabla_{X}^{1} \epsilon_{1}-\alpha^{2}\left(Y, \nabla_{X}^{1} \epsilon_{1}\right) \\
& -\alpha^{1}\left(Y, \alpha^{2}\left(X, \epsilon_{1}\right)\right)-\nabla_{Y}^{2} \alpha^{2}\left(X, \epsilon_{1}\right) \\
& \left.-\nabla_{[X, Y]}^{1} \epsilon_{1}-\alpha^{2}\left([X, Y], \epsilon_{1}\right), \eta_{1}\right) \\
= & g^{1}\left(R^{1}(X, Y) \epsilon_{1}, \eta_{1}\right)+g^{2}\left(\alpha^{2}\left(X, \epsilon_{1}\right), \alpha^{2}\left(Y, \eta_{1}\right)\right) \\
& -g^{2}\left(\alpha^{2}\left(X, \eta_{1}\right), \alpha^{2}\left(Y, \epsilon_{1}\right)\right),
\end{aligned}
$$

que é a equação (4.4). A equação (4.5) segue de forma análoga. Quanto à equação (4.6), temos:

$$
\begin{aligned}
g\left(R(X, Y) \epsilon_{1}, \eta_{2}\right)= & g\left(\alpha^{2}\left(X, \nabla_{Y}^{1} \epsilon_{1}\right)+\nabla_{X}^{2} \alpha^{2}\left(Y, \epsilon_{1}\right)-\alpha^{2}\left(Y, \nabla_{X}^{1} \epsilon_{1}\right)\right. \\
& \left.-\nabla_{Y}^{2} \alpha^{2}\left(X, \epsilon_{1}\right)-\alpha^{2}\left([X, Y], \epsilon_{1}\right), \eta_{2}\right) \\
= & g\left(\alpha^{2}\left(X, \nabla_{Y}^{1} \epsilon_{1}\right)+\nabla_{X}^{2} \alpha^{2}\left(Y, \epsilon_{1}\right)-\alpha^{2}\left(Y, \nabla_{X}^{1} \epsilon_{1}\right)\right. \\
& -\nabla_{Y}^{2} \alpha^{2}\left(X, \epsilon_{1}\right)+\alpha^{2}\left(\nabla_{Y}^{M} X, \epsilon_{1}\right)-\alpha^{2}\left(\nabla_{X}^{M} Y, \epsilon_{1}\right) \\
& \left.+\alpha^{2}\left(T(X, Y), \epsilon_{1}\right), \eta_{2}\right) \\
= & g^{2}\left(\left(\nabla_{X}^{2} \alpha^{2}\right)\left(Y, \epsilon_{1}\right), \eta_{2}\right)-g^{2}\left(\left(\nabla_{Y}^{2} \alpha^{2}\right)\left(X, \epsilon_{1}\right), \eta_{2}\right) \\
& +g^{2}\left(\alpha^{2}\left(T(X, Y), \epsilon_{1}\right), \eta_{2}\right) .
\end{aligned}
$$


A equação (4.7) segue de forma análoga. Finalmente, quanto à equação (4.8), temos:

$$
\begin{aligned}
g\left(T^{\iota}(X, Y), \epsilon_{1}\right)= & g\left(\nabla_{X} \iota(Y)-\nabla_{Y} \iota(X)-\iota([X, Y]), \epsilon_{1}\right) \\
= & g\left(\nabla_{X}^{1} \iota_{1}(Y)+\alpha^{1}\left(X, \iota_{2}(Y)\right)+\nabla_{X}^{2} \iota_{2}(Y)+\alpha^{2}\left(X, \iota_{1}(Y)\right)\right. \\
& -\nabla_{Y}^{1} \iota_{1}(X)-\alpha^{1}\left(Y, \iota_{2}(X)\right)-\nabla_{Y}^{2} \iota_{2}(X)-\alpha^{2}\left(Y, \iota_{1}(X)\right) \\
& \left.-\iota([X, Y]), \epsilon_{1}\right) \\
= & g^{1}\left(T^{\iota}(X, Y), \epsilon_{1}\right)+g^{1}\left(\alpha^{1}\left(X, \iota_{2}(Y)\right), \epsilon_{1}\right) \\
& -g^{1}\left(\alpha^{1}\left(Y, \iota_{2}(X)\right), \epsilon_{1}\right) .
\end{aligned}
$$

A equação (4.9) segue de forma inteiramente análoga.

A equação (4.4) é chamada a equação generalizada de Gauss, (4.5) é chamada a equação generalizada de Ricci, (4.6) e (4.7) são as equações generalizadas de Codazzi e, (4.8) e (4.9) são as equações generalizadas de torção.

\subsection{As equações fundamentais}

Sejam $\left(M^{n}, g\right),\left(\bar{M}^{n+k}, \bar{g}\right)$ variedades semi-Riemannianas com suas respectivas conexões de Levi-Civita $\nabla$ e $\bar{\nabla}, E$ um fibrado vetorial sobre $M$ munido de uma métrica semi-Riemanniana $g^{E}, \nabla^{E}$ uma conexão em $E$ compatível com $g^{E}$ e $\alpha$ uma seção diferenciável simétrica de $\operatorname{Lin}_{2}(T M ; E)$.

Definição 4.3.1 Uma imersão isométrica de $M$ em $\bar{M}$ é um par $(f, S)$, onde $f: M \rightarrow \bar{M}$ é uma imersão isométrica e $S: E \rightarrow T M_{f}^{\perp}$ é uma isometria de fibrados vetoriais tal que

$$
S(\alpha(X, Y))=\alpha^{f}(X, Y) \text { e } S\left(\nabla_{X}^{E} \epsilon\right)=\nabla_{X}^{\perp} S(\epsilon),
$$

para quaisquer $X, Y \in \boldsymbol{\Gamma}(T M)$ e $\epsilon \in \boldsymbol{\Gamma}(E)$, onde $T M_{f}^{\perp}, \nabla^{\perp}, \alpha^{f}$ denotam o fibrado normal, a conexão normal e a segunda forma fundamental de $f$, respectivamente.

Observação 4.3.2 Quando nos referirmos a uma imersão isométrica $(f, S)$ de $M$ em $\bar{M}$, estaremos levando em consideração todos os dados prescritos na Definição 4.3.1. 
Analisaremos agora as equações generalizadas no contexto de imersões isométricas. Mais precisamente, seja $(f, S)$ uma imersão isométrica de $(M, g)$ em $(\bar{M}, \bar{g})$. Então, como $\nabla, \nabla^{\perp}$ e $\alpha^{f}$ são as componentes da conexão $\bar{\nabla}$, relativo à decomposição $\left.T \bar{M}\right|_{f(M)}=T M \oplus T M_{f}^{\perp}$, a equação (4.4) fornece:

$$
\begin{array}{r}
\bar{g}\left(\bar{R}_{f(x)}\left(\mathrm{d} f_{x}(X), \mathrm{d} f_{x}(Y)\right) \mathrm{d} f_{x}(Z), \mathrm{d} f_{x}(W)\right)=g\left(R_{x}(X, Y) Z, W\right) \\
+g^{\perp}\left(\alpha^{f}(X, Z), \alpha^{f}(Y, W)\right)-g^{\perp}\left(\alpha^{f}(X, W), \alpha^{f}(Y, Z)\right),
\end{array}
$$

para quaisquer $x \in M$ e $X, Y, Z, W \in T_{x} M$, onde $R$ e $\bar{R}$ denotam os tensores de curvatura de $\nabla$ e $\bar{\nabla}$, respectivamente. A equação (4.10) é chamada a equação de Gauss da imersão isométrica $f$. No caso de imersões isométricas, fazendo $\nabla^{M}=\nabla$, as equações (4.6) e (4.7) são equivalentes. Assim, a equação (4.6) fornece:

$$
\begin{aligned}
\bar{g}\left(\bar{R}_{f(x)}\left(\mathrm{d} f_{x}(X), \mathrm{d} f_{x}(Y)\right) \mathrm{d} f_{x}(Z), \eta\right)= & g^{\perp}\left(\left(\nabla_{X}^{\perp} \alpha^{f}\right)(Y, Z), \eta\right) \\
& -g^{\perp}\left(\left(\nabla_{Y}^{\perp} \alpha^{f}\right)(X, Z), \eta\right),
\end{aligned}
$$

para quaisquer $x \in M, X, Y, Z \in T_{x} M$ e $\eta \in\left(T_{x} M\right)^{\perp}$. A equação (4.11) é chamada a equação de Codazzi da imersão isométrica $f$. A equação (4.5) fornece:

$$
\begin{array}{r}
\bar{g}\left(\bar{R}_{f(x)}\left(\mathrm{d} f_{x}(X), \mathrm{d} f_{x}(Y)\right) \eta, \xi\right)=g^{\perp}\left(R_{x}^{\perp}(X, Y) \eta, \xi\right) \\
+g(A(X, \eta), A(Y, \xi))-g(A(X, \xi), A(Y, \eta)),
\end{array}
$$

para quaisquer $x \in M, X, Y \in T_{x} M$ e $\eta, \xi \in\left(T_{x} M\right)^{\perp}$, onde $R^{\perp}$ denota o tensor de curvatura da conexão normal $\nabla^{\perp}$. A equação (4.12) é chamada a equação de Ricci da imersão isométrica $f$. As equações (4.8) e (4.9), neste caso, são trivialmente satisfeitas.

Observação 4.3.3 Seja $(f, S)$ uma imersão isométrica de $M$ em $\bar{M}$. Para cada $x \in M$, existe uma vizinhança $U$ de $x$ em $M$ tal que $\left.f\right|_{U}$ é um mergulho. Assim, podemos identificar $U$ com $f(U)$ e, consequentemente, identificar $\mathrm{d} f_{x}(X)$ com $X$, para quaisquer $x \in U$ e $X \in T_{x} M$. Dessa forma, podemos considerar $T_{x} M$ como um subespaço de $T_{x} \bar{M}$ e escrever

$$
T_{x} \bar{M}=T_{x} M \oplus\left(T_{x} M\right)^{\perp},
$$

onde $\left(T_{x} M\right)^{\perp}$ denota o complemento ortogonal de $T_{x} M$ em $T_{x} \bar{M}$. 
Exemplo 4.3.4 Suponha que o espaço ambiente $\bar{M}$ tenha curvatura seccional constante igual a $c$. Neste caso, o tensor de curvatura $\bar{R}$ de $\bar{M}$ é dado por

$$
\bar{R}(X, Y)=c X \wedge Y,
$$

para quaisquer $X, Y \in \boldsymbol{\Gamma}(T M)$ (ver [4, Lema 4.3.4]) onde, para todo $Z \in$ $\Gamma(T M), X \wedge Y$ é dado por:

$$
(X \wedge Y) Z=g(X, Z) Y-g(Y, Z) X .
$$

Então, para $X, Y, Z, W \in \boldsymbol{\Gamma}(T M)$ e $\eta, \xi \in \boldsymbol{\Gamma}\left(T M_{f}^{\perp}\right)$, as equações de Gauss, Codazzi e Ricci são:

$$
\begin{aligned}
g(R(X, Y) Z, W)= & c g((X \wedge Y) Z, W)+g^{\perp}\left(\alpha^{f}(X, W), \alpha^{f}(Y, Z)\right) \\
& -g^{\perp}\left(\alpha^{f}(X, Z), \alpha^{f}(Y, W)\right) \\
& \left(\nabla_{X}^{\perp} \alpha^{f}\right)(Y, Z)=\left(\nabla_{Y}^{\perp} \alpha^{f}\right)(X, Z), \\
g^{\perp}\left(R^{\perp}(X, Y) \eta, \xi\right)= & g(A(X, \xi), A(Y, \eta))-g(A(X, \eta), A(Y, \xi)),
\end{aligned}
$$

respectivamente.

Exemplo 4.3.5 Suponha que a codimensão da imersão seja 1. Assim, $\nabla_{X}^{\perp} \eta=0$, para quaisquer $X \in \boldsymbol{\Gamma}(T M)$ e $\eta \in \boldsymbol{\Gamma}\left(T M_{f}^{\perp}\right)$. De fato, neste caso, podemos escolher, localmente, um campo normal unitário para $M$ : em uma vizinhança $U$ de um ponto $x \in M$, escolha $N$ tal que $\bar{g}(N, N)=1 \mathrm{e}$ $N(y) \in\left(T_{y} M\right)^{\perp}$, para todo $y \in U$. Isso é possível pois existem somente duas escolhas para $N$. Assim, para todo $X \in \boldsymbol{\Gamma}(T M)$, temos:

$$
0=X \bar{g}(N, N)=2 \bar{g}\left(\nabla_{X}^{\perp} N, N\right),
$$

$\operatorname{logo} \nabla_{X}^{\perp} N \in \boldsymbol{\Gamma}(T M)$, pois $\operatorname{rank}\left(T M_{f}^{\perp}\right)=1$. Associado ao campo normal unitário $N$, definimos o operador de Weingarten $A: \boldsymbol{\Gamma}(T M) \rightarrow \boldsymbol{\Gamma}(T M)$ dado por

$$
g(A X, Y)=g^{\perp}\left(\alpha^{f}(X, Y), N\right),
$$

para quaisquer $X, Y \in \boldsymbol{\Gamma}(T M)$. Assim, neste caso, as equações de Gauss e Codazzi são, respectivamente:

$$
\begin{gathered}
\bar{R}(X, Y) Z=R(X, Y) Z+(A X \wedge A Y) Z, \\
\bar{R}(X, Y) N=\left(\nabla_{X} A\right) Y-\left(\nabla_{Y} A\right) X .
\end{gathered}
$$

A equação de Ricci é trivialmente satisfeita. 


\subsection{Variedades Infinitesimalmente homogêneas}

Sejam $M^{n}$ uma variedade diferenciável, $G$ um subgrupo de Lie de GL $\left(\mathbb{R}^{n}\right)$ e assuma que $M$ está munida de uma conexão $\nabla$ e uma $G$-estrutura $P \subset$ $\operatorname{FR}(T M)$. Consideremos, para cada $x \in M$, o subgrupo de Lie $G_{x}$ de $\mathrm{GL}\left(T_{x} M\right)$ dos endomorfismos de $T_{x} M$ que preservam $G$-estrutura, e $\mathfrak{g}_{x} \subset$ $\mathfrak{g l}\left(T_{x} M\right)$ a álgebra de Lie de $G_{x}$. Dados $x, y \in M$ e $\sigma: T_{x} M \rightarrow T_{y} M$ uma aplicação que preserva $G$-estrutura, o isomorfismo de grupos de Lie

$$
\mathcal{I}_{\sigma}: \mathrm{GL}\left(T_{x} M\right) \rightarrow \mathrm{GL}\left(T_{y} M\right)
$$

definido por $\mathcal{I}_{\sigma}(T)=\sigma \circ T \circ \sigma^{-1}$, para todo $T \in \operatorname{GL}\left(T_{x} M\right)$, transforma $G_{x}$ sobre $G_{y}$. Sua diferencial no elemento identidade:

$$
\operatorname{Ad}_{\sigma}: \mathfrak{g l}\left(T_{x} M\right) \rightarrow \mathfrak{g l}\left(T_{y} M\right)
$$

transforma $\mathfrak{g}_{x}$ sobre $\mathfrak{g}_{y}$ e, portanto, induz um isomorfismo linear

$$
\overline{\operatorname{Ad}}_{\sigma}: \mathfrak{g l}\left(T_{x} M\right) / \mathfrak{g}_{x} \rightarrow \mathfrak{g l}\left(T_{y} M\right) / \mathfrak{g}_{y} .
$$

Denotemos por $R$ e $T$ os tensores de curvatura e torção da conexão $\nabla$, respectivamente, e seja $\mathfrak{I}_{x}^{P}: T_{x} M \rightarrow \mathfrak{g l}\left(T_{x} M\right) / \mathfrak{g}_{x}$ a inner torsion da $G$ estrutura $P$, com $x \in M$.

Definição 4.4.1 Dizemos que a terna $(M, \nabla, P)$ é uma variedade infinitesimalmente homogênea se, para quaisquer $x, y \in M$, toda aplicação $\sigma$ : $T_{x} M \rightarrow T_{y} M$ que preserva $G$-estrutura relaciona $R_{x} \operatorname{com} R_{y}, T_{x}$ com $T_{y} \mathrm{e}$ $\mathfrak{I}_{x}^{P} \operatorname{com} \mathfrak{I}_{y}^{P}$, ou seja,

$$
\begin{aligned}
R_{y}(\sigma \cdot, \sigma \cdot) & =\sigma \circ R_{x}(\cdot, \cdot) \circ \sigma^{-1}, \\
T_{y}(\sigma \cdot, \sigma \cdot) & =\sigma \circ T_{x}, \\
\mathfrak{I}_{y}^{P} \circ \sigma & =\overline{\operatorname{Ad}}_{\sigma} \circ \mathfrak{I}_{x}^{P} .
\end{aligned}
$$

Observação 4.4.2 A condição de homogeneidade infinitesimal significa que a curvatura, a torção e a inner torsion são constantes nos referenciais que pertencem a $G$-estrutura $P$.

O Lema seguinte estabelece, precisamente, a Observação 4.4.2. 
Lema 4.4.3 Seja $(M, \nabla)$ uma variedade afim munida de uma $G$-estrutura $P \subset \operatorname{FR}(T M)$, onde $G$ é um subgrupo de Lie de $\operatorname{GL}\left(\mathbb{R}^{n}\right)$. Então, $(M, \nabla, P)$ é infinitesimalmente homogênea se, e somente se, existem aplicações bilineares $R_{\mathrm{O}}: \mathbb{R}^{n} \times \mathbb{R}^{n} \rightarrow \mathfrak{g l}\left(\mathbb{R}^{n}\right), T_{\mathrm{o}}: \mathbb{R}^{n} \times \mathbb{R}^{n} \rightarrow \mathbb{R}^{n}$, e uma aplicação linear $\mathfrak{I}_{\mathrm{O}}: \mathbb{R}^{n} \rightarrow \mathfrak{g l}\left(\mathbb{R}^{n}\right) / \mathfrak{g}$ tais que:

$$
\begin{array}{r}
p^{-1} \circ R_{x}(p \cdot, p \cdot) \circ p=R_{\mathrm{o}}, \\
p^{-1} \circ T_{x}(p \cdot, p \cdot)=T_{\mathrm{o}}, \\
\overline{\operatorname{Ad}}_{p} \circ \mathfrak{I}_{\mathrm{o}}=\mathfrak{I}_{x}^{P} \circ p,
\end{array}
$$

para todo $x \in M$ e para todo $p \in P_{x}$.

Demonstração. Assuma a existência de $R_{\mathrm{o}}, T_{\mathrm{o}}, \mathfrak{I}_{\mathrm{o}}$ tais que (4.18) vale para todo $x \in M$ e para todo $P \in P_{x}$. Sejam $x, y \in M$ e $\sigma: T_{x} M \rightarrow T_{y} M$ uma aplicação que preserva $G$-estrutura fixados. Dado $p \in P_{x}$, seja $q=$ $\sigma \circ p \in P_{y}$. Então:

$$
p^{*} R_{x}=R_{\mathrm{o}}=q^{*} R_{y}=p^{*} \sigma^{*} R_{x}
$$

e, assim, $R_{x}=\sigma^{*} R_{y}$, i.e., $\sigma$ relaciona $R_{x} \operatorname{com} R_{y}$. De forma análoga se mostra que $\sigma$ relaciona $T_{x} \operatorname{com} T_{y}$. Além disso, $\overline{\operatorname{Ad}}_{p} \circ \mathfrak{I}_{\mathrm{o}}=\mathfrak{I}_{x}^{P} \circ p, \overline{\operatorname{Ad}}_{q} \circ \mathfrak{I}_{\mathrm{o}}=$ $\mathfrak{I}_{y}^{P} \circ q$ e, portanto:

$$
\mathfrak{I}_{y}^{P} \circ \sigma \circ p=\mathfrak{I}_{y}^{P} \circ q=\overline{\operatorname{Ad}}_{q} \circ \mathfrak{I}_{\mathrm{o}}=\overline{\operatorname{Ad}}_{\sigma} \circ \overline{\operatorname{Ad}}_{p} \circ \mathfrak{I}_{\mathrm{o}}=\overline{\operatorname{Ad}}_{\sigma} \circ \mathfrak{I}_{x}^{P} \circ p,
$$

provando $\overline{\operatorname{Ad}}_{\sigma} \circ \mathfrak{I}_{x}^{P}=\mathfrak{I}_{y}^{P} \circ \sigma$. Reciprocamente, assuma que $(M, \nabla, P)$ é infinitesimalmente homogênea. Sejam $x \in M$ e $p \in P_{x}$ arbitrários e defina:

$$
R_{\mathrm{o}}=p^{*} R_{x}, \quad T_{\mathrm{o}}=p^{*} T_{x}, \quad \mathfrak{I}_{\mathrm{o}}=\left(\overline{\operatorname{Ad}}_{p}\right)^{-1} \circ \mathfrak{I}_{x}^{P} \circ p .
$$

Dados $y \in M$ e $q \in P_{y}$, a aplicação $\sigma=q \circ p^{-1}: T_{x} M \rightarrow T_{y} M$ é uma aplicação que preserva $G$-estrutura e, portanto, $\sigma^{*} R_{y}=R_{x}, \sigma^{*} T_{y}=T_{x}$ e $\overline{\operatorname{Ad}}_{\sigma} \circ \mathfrak{I}_{x}^{P}=\mathfrak{I}_{y}^{P} \circ \sigma$. Assim:

$$
q^{*} R_{y}=p^{*} \sigma^{*} R_{y}=p^{*} R_{x}=R_{\mathrm{o}}, \quad q^{*} T_{y}=p^{*} \sigma^{*} T_{y}=p^{*} T_{x}=T_{\mathrm{o}} ;
$$

além disso, temos:

$$
\overline{\operatorname{Ad}}_{q} \circ \mathfrak{I}_{\mathrm{o}}=\overline{\operatorname{Ad}}_{q} \circ\left(\overline{\operatorname{Ad}}_{p}\right)^{-1} \circ \mathfrak{I}_{x}^{P} \circ p=\overline{\operatorname{Ad}}_{\sigma} \circ \mathfrak{I}_{x}^{P} \circ p=\mathfrak{I}_{y}^{P} \circ \sigma \circ p=\mathfrak{I}_{y}^{P} \circ q,
$$

concluindo a prova.

O Lema 4.4.3 afirma que uma variedade afim com $G$-estrutura $(M, \nabla, P)$ é infinitesimalmente homogênea se podemos descrever a inner torsion $\mathfrak{I}^{P}$, o 
tensor de curvatura $R$ e o tensor de torção $T$ por fórmulas que envolvem somente os elementos da $G$-estrutura. As aplicações $R_{\mathrm{o}}, T_{\mathrm{o}}$ e $\mathfrak{I}_{\mathrm{o}}$, dadas no Lema 4.4.3, são chamadas os tensores característicos da variedade infinitesimalmente homogênea $(M, \nabla, P)$.

Observação 4.4.4 Seja $(M, \nabla, P)$ uma variedade infinitesimalmente homogênea, com tensores característicos $R_{\mathrm{o}}, T_{\mathrm{o}}, \mathfrak{I}_{\mathrm{o}}$. Claramente, os tensores $R_{\mathrm{o}}, T_{\mathrm{o}}$ e $\mathfrak{I}_{\mathrm{o}}$ são invariantes pela ação do grupo $G$. Esse fato implica que podemos induzir "versões"dos tensores $R_{\mathrm{o}}, T_{\mathrm{o}}, \mathfrak{I}_{\mathrm{o}}$ em qualquer espaço vetorial real munido de uma $G$-estrutura. Mais precisamente, seja $V$ um espaço vetorial real de dimensão $n$ munido de uma $G$-estrutura $P_{V} \subset \mathrm{FR}(V)$. Denotemos por $G_{V} \subset \mathrm{GL}(V)$ o grupo de Lie dos automorfismos de $V$ que preservam $G$-estrutura e por $\mathfrak{g}_{V} \subset \mathfrak{g l}(V)$ sua álgebra de Lie. Dado um referencial arbitrário $p \in P_{V}$, existe uma única terna de tensores $R_{V}: V \times V \rightarrow \mathfrak{g l}(V)$, $T_{V}: V \times V \rightarrow V, \mathfrak{I}_{V}: V \rightarrow \mathfrak{g l}(V) / \mathfrak{g}_{V}$ que são relacionados a $R_{\mathrm{o}}, T_{\mathrm{o}}$ e $\mathfrak{I}_{\mathrm{o}}$, respectivamente, por $p$. De fato, defina $R_{V}, T_{V}, \mathfrak{I}_{V}$ pelo push-forward de $R_{\mathrm{o}}, T_{\mathrm{o}}$ e $\mathfrak{I}_{\mathrm{o}}$, respectivamente, i.e.,

$$
R_{V}=p_{*} R_{\mathrm{o}}, \quad T_{V}=p_{*} T_{V}, \quad \Im_{V}=p^{-1} \circ \overline{\mathrm{Ad}}_{p} \circ \Im_{\mathrm{o}} .
$$

A $G$-invariância de $R_{\mathrm{o}}, T_{\mathrm{o}}, \mathfrak{I}_{\mathrm{o}}$ implica que $R_{V}, T_{V}, \mathfrak{I}_{V}$ não dependem da escolha do referencial $p \in P_{V}$.

Seja $(M, \nabla)$ uma variedade afim munida de uma $G$-estrutura $P$, onde $G$ é um subgrupo de Lie de $\operatorname{GL}\left(\mathbb{R}^{n}\right)$.

Definição 4.4.5 Dizemos que a terna $(M, \nabla, P)$ é uma variedade localmente $G$-homogênea se, para quaisquer $x, y \in M$ e para toda aplicação $\sigma: T_{x} M \rightarrow$ $T_{y} M$ que preserva $G$-estrutura, existem uma vizinhança $U$ de $x$ em $M$, uma vizinhança $V$ de $y$ em $M$ e um difeomorfismo afim $f: U \rightarrow V$ que preserva $G$-estrutura tal que $f(x)=y$ e $\mathrm{d} f_{x}=\sigma$. Se $U=V=M$, dizemos que a terna $(M, \nabla, P)$ é uma variedade $G$-homogênea.

Lema 4.4.6 Toda variedade (localmente) $G$-homogênea é infinitesimalmente homogênea.

Demonstração. Isso segue do fato de que os tensores de curvatura e torção, bem como a inner torsion, são invariantes por difeomorfismos afins que preservam $G$-estrutura.

A recíproca do Lema 4.4.6 também é verdadeira. Mais precisamente, temos o seguinte: 
Lema 4.4.7 Seja $(M, \nabla, P)$ uma variedade infinitesimalmente homogênea. Então $(M, \nabla, P)$ é localmente $G$-homogênea. Se, além disso, $(M, \nabla)$ é geodesicamente completa e $M$ é conexa e simplesmente conexa, então $(M, \nabla, P)$ é $G$-homogênea.

A prova do Lema 4.4.7 consiste em usar o Teorema de Cartan-AmbroseHicks $^{1}$ para exibir, para quaisquer $x, y \in M$ e para cada aplicação que preserva $G$-estrutura $\sigma: T_{x} M \rightarrow T_{y} M$, um difeomorfismo afim $f: U \rightarrow V$ que preserva $G$-estrutura tal que $f(x)=y$ e $\mathrm{d} f_{x}=\sigma$, onde $U$ é uma vizinhança de $x$ e $V$ é uma vizinhança de $y$. Para maiores detalhes, ver [26, Proposição 6.4].

Veremos a seguir alguns exemplos de variedades infinitesimalmente homogêneas.

Exemplo 4.4.8 Sejam $\left(M^{n}, g\right)$ uma variedade semi-Riemanniana de índice $r$ e de curvatura seccional constante igual a $c$ e $\nabla$ a conexão de Levi-Civita de $(M, g)$. Então, o tensor de curvatura $R$ de $\nabla$ é dado por:

$$
R_{x}(X, Y) Z=c(X \wedge Y) Z,
$$

para quaisquer $x \in M$ e $X, Y, Z \in T_{x} M$. Se $P=\mathrm{FR}^{\mathrm{o}}(T M)$ é a $\mathrm{O}_{r}\left(\mathbb{R}^{n}\right)$ estrutura em $M$ consistindo de todos os referenciais ortonormais então a terna $(M, \nabla, P)$ é infinitesimalmente homogênea. De fato, como $\nabla$ é a conexão de Levi-Civita de $(M, g)$, o tensor de torção $T$ de $\nabla$ é nulo. Além disso, do Exemplo 3.3.9, temos $\mathfrak{I}^{P}=0$. A expressão (4.19) implica que $R$ é constante nos referenciais que pertencem a $\mathrm{O}_{r}\left(\mathbb{R}^{n}\right)$-estrutura (o tensor de curvatura $R$ pode ser descrito usando-se somente a $\mathrm{O}_{r}\left(\mathbb{R}^{n}\right)$-estrutura $P$, que pode ser identificado com o tensor métrico $g$ ). Assim, neste caso, os tensores característicos $R_{\mathrm{o}}, T_{\mathrm{o}}, \mathfrak{I}_{\mathrm{o}}$ são dados por $T_{\mathrm{o}}=0, \mathfrak{I}_{\mathrm{o}}=0 \mathrm{e}$

$$
R_{\mathrm{o}}(X, Y) Z=c(\langle X, Z\rangle Y-\langle Y, Z\rangle X),
$$

para quaisquer $X, Y, Z \in \mathbb{R}^{n}$, onde $\langle\cdot, \cdot\rangle$ denota a forma bilinear de Minkowski em $\mathbb{R}^{n}$ de índice $r$. Finalmente, se $V$ é um espaço vetorial real de dimensão $n$ munido de um produto escalar $\langle\cdot, \cdot\rangle$ de índice $r$, então as "versões" $R_{V}, T_{V}$, $\mathfrak{I}_{V}$ dos tensores característicos de $(M, \nabla, P)$ são dados por $T_{V}=0, \mathfrak{I}_{V}=0$ e

$$
R_{V}(X, Y) Z=c(\langle X, Z\rangle Y-\langle Y, Z\rangle X)
$$

para quaisquer $X, Y, Z \in V$.

\footnotetext{
${ }^{1}$ Para maiores detalhes sobre o Teorema de Cartan-Ambrose-Hicks, ver [37, Teorema 1.9.1].
} 
Exemplo 4.4.9 Sejam $\left(M_{1}^{n_{1}}, \nabla^{1}, P^{1}\right)$ e $\left(M_{2}^{n_{2}}, \nabla^{2}, P^{2}\right)$ variedades infinitesimalmente homogêneas, onde o grupo $G_{i}$, que determina a $G_{i}$-estrutura $P^{i}$ na variedade $M_{i}$, é um subgrupo de Lie de $\operatorname{GL}\left(\mathbb{R}^{n_{i}}\right), i=1,2$. Consideremos a variedade produto $M=M_{1} \times M_{2}$. Seja $\nabla$ a conexão em $M$ induzida por $\nabla^{1}$ e $\nabla^{2}$, ou seja, $\nabla$ é a única conexão em $M$ tal que, para quaisquer $X_{1}, Y_{1} \in \boldsymbol{\Gamma}\left(T M_{1}\right)$ e $X_{2}, Y_{2} \in \boldsymbol{\Gamma}\left(T M_{2}\right)$, tem-se:

$$
\nabla_{X_{1}+X_{2}} Y_{1}+Y_{2}=\nabla_{X_{1}}^{1} Y_{1}+\nabla_{X_{2}}^{2} Y_{2} .
$$

O produto $G=G_{1} \times G_{2}$ pode ser identificado, diagonalmente, com um subgrupo de Lie de $\operatorname{GL}\left(\mathbb{R}^{n_{1}+n_{2}}\right)$. Consideremos o produto $P=P^{1} \times P^{2}$. Mais precisamente, dado $x=\left(x_{1}, x_{2}\right) \in M$, a fibra $P_{x}$ é o conjunto de todos os referenciais $p: \mathbb{R}^{n} \rightarrow T_{x} M$ da forma $p=p_{1} \oplus p_{2}$, onde $p_{i}: \mathbb{R}^{n_{i}} \rightarrow T_{x_{i}} M_{i}$ é um referencial da fibra $P_{x_{i}}, i=1,2$ e $n=n_{1}+n_{2}$. Segue, então, que $P$ é uma $G$-estrutura em $M$ e a terna $(M, \nabla, P)$ é infinitesimalmente homogênea. De fato, os tensores característicos de $(M, \nabla, P)$ são dados pelas somas diretas ortogonais dos tensores característicos de $\left(M_{1}, \nabla^{1}, P^{1}\right)$ e $\left(M_{2}, \nabla^{2}, P^{2}\right)$. Além disso, se $V$ é um espaço vetorial real de dimensão $n$, então uma $G$-estrutura em $V$ é determinada por uma decomposição em soma direta $V=V_{1} \oplus V_{2}$, com $\operatorname{dim}\left(V_{i}\right)=n_{i}, i=1,2$, por uma $G_{1}$-estrutura em $V_{1}$ e por uma $G_{2}$-estrutura em $V_{2}$.

Exemplo 4.4.10 Um caso particular do Exemplo 4.4.9 é quando $\left(M_{1}, g^{1}\right)$, $\left(M_{2}, g^{2}\right)$ são variedades semi-Riemannianas de curvatura seccional constante iguais a $c_{1}$ e $c_{2}$, respectivamente. Consideremos a variedade produto $M=$ $M_{1} \times M_{2}$ munida da métrica semi-Riemanniana $g$ dada pela soma direta ortogonal de $g^{1}$ e $g^{2}$, i.e.:

$$
g_{\left(x_{1}, x_{2}\right)}\left(\left(X_{1}, X_{2}\right),\left(Y_{1}, Y_{2}\right)\right)=g_{x_{1}}^{1}\left(X_{1}, X_{2}\right)+g_{x_{2}}^{2}\left(X_{2}, Y_{2}\right),
$$

para quaisquer $x_{i} \in M_{i}$ e $X_{i}, Y_{i} \in T_{x_{i}} M_{i}, i=1,2$. O tensor de curvatura $R$ da conexão de Levi-Civita $\nabla$ de $(M, g)$ é dado por:

$$
R_{x}\left(\left(X_{1}, X_{2}\right),\left(Y_{1}, Y_{2}\right)\right)\left(Z_{1}, Z_{2}\right)=R_{x_{1}}^{1}\left(X_{1}, Y_{1}\right) Z_{1}+R_{x_{2}}^{2}\left(X_{2}, Y_{2}\right) Z_{2},
$$

para quaisquer $x=\left(x_{1}, x_{2}\right) \in M$ e $X_{i}, Y_{i}, Z_{i} \in T_{x_{i}} M_{i}, i=1,2$, onde $R^{1}$, $R^{2}$ denotam os tensores de curvatura das conexões de Levi-Citiva $\nabla^{1}$ e $\nabla^{2}$, respectivamente. Consideremos o conjunto

$$
P=\mathrm{FR}^{\mathrm{o}}\left(T M ; \mathbb{R}^{n_{1}} \oplus\{0\}^{n_{2}}, \operatorname{pr}_{1}^{*}\left(T M_{1}\right)\right),
$$

onde $\operatorname{pr}_{1}: M \rightarrow M_{1}$ denota a projeção sobre o primeiro fator e $\mathbb{R}^{n}=$ $\mathbb{R}^{n_{1}} \oplus \mathbb{R}^{n_{2}}, n=n_{1}+n_{2}$, está munido da soma ortogonal das formas bilineares 
de Minkowski de mesmos índices de $g^{1}$ e $g^{2}$. De forma mais explícita, para todo $x=\left(x_{1}, x_{2}\right) \in M$, temos:

$$
P_{x}=\left\{p \in \mathrm{FR}^{\mathrm{o}}\left(T_{x} M\right): p\left(\mathbb{R}^{n_{1}} \oplus\{0\}^{n_{2}}\right)=T_{x_{1}} M_{1} \oplus\{0\}\right\} .
$$

Automaticamente, tem-se:

$$
p\left(\{0\}^{n_{1}} \oplus \mathbb{R}^{n_{2}}\right)=\{0\} \oplus T_{x_{2}} M_{2},
$$

para todo $p \in P_{x}$. Então, $P$ é uma $G$-estrutura em $M$ com

$$
G=\mathrm{O}_{r}\left(\mathbb{R}^{n} ; \mathbb{R}^{n_{1}}\right) \simeq \mathrm{O}_{r_{1}}\left(\mathbb{R}^{n_{1}}\right) \times \mathrm{O}_{r_{2}}\left(\mathbb{R}^{n_{2}}\right) .
$$

A terna $(M, \nabla, P)$ é infinitesimalmente homogênea. De fato, como $\nabla$ é compatível com $g$ e a derivada covariante de seções de $\operatorname{pr}_{1}^{*}\left(T M_{1}\right)$ são seções de $\operatorname{pr}_{1}^{*}\left(T M_{1}\right)$, segue do Exemplo 3.3.10 que a inner torsion $\mathfrak{I}^{P}$ é nula. Além disso, o tensor de torção $T$ de $\nabla$ é nulo e a fórmula (4.20) implica que o tensor de curvatura $R$ é constante nos referenciais que pertencem a $G$-estrutura $P$.

Exemplo 4.4.11 Sejam $K$ um grupo de Lie de dimensão $n, \mathfrak{k}$ sua álgebra de Lie e $\nabla$ uma conexão invariante à esquerda em $K$, i.e., as translações à esquerda de $K$ são aplicações afins. Fixemos um isomorfismo linear $p_{0}$ : $\mathbb{R}^{n} \rightarrow \mathfrak{k}$ e definimos uma seção global diferenciável $s: K \rightarrow \mathrm{FR}(T K)$ por:

$$
s(g)=\mathrm{d} L_{g}(1) \circ p_{0} \in \mathrm{FR}\left(T_{g} K\right),
$$

para todo $g \in K$. Então, $P=s(K)$ é uma $G$-estrutura em $K$, com $G=\left\{\operatorname{Id}_{\mathbb{R}^{n}}\right\}$. Como as translações à esquerda são difeomorfismos afins que preservam $G$-estrutura, segue que $(K, \nabla, P)$ é $G$-homogênea e, pelo Lema 4.4.6, $(K, \nabla, P)$ é infinitesimalmente homogênea. A fim de explicitar os tensores característicos de $(K, \nabla, P)$, denotemos por $\lambda: \mathfrak{k} \rightarrow \mathfrak{g l}(\mathfrak{k})$ a aplicação linear correspondente à conexão $\nabla$, dada pelo Lema 2.6.2. Pelo Exemplo 3.3.8, a inner torsion $\mathfrak{I}_{g}^{P}$ coincide com o tensor de Christoffel $\Gamma_{g}: T_{g} K \rightarrow \mathfrak{g l}\left(T_{g} K\right)$. Observe que, no elemento identidade $1 \in K$, o tensor de Christoffel $\Gamma_{1}$ coincide com a aplicação linear $\lambda$. Assim, $\mathfrak{I}_{1}^{P}=\lambda$. Além disso, os tensores de curvatura e torção da conexão $\nabla$ no elemento identidade $1 \in K$ são dados por:

$$
R_{1}(X, Y)=[\lambda(X), \lambda(Y)]-\lambda([X, Y])
$$

e

$$
T_{1}(X, Y)=\lambda(X) Y-\lambda(Y) X-[X, Y],
$$


respectivamente, para quaisquer $X, Y \in \mathfrak{k}$. Assim, os tensores característicos $R_{\mathrm{o}}, T_{\mathrm{o}}, \mathfrak{I}_{\mathrm{o}}$ de $(K, \nabla, P)$ são dados por:

$$
\begin{aligned}
R_{\mathrm{o}}(X, Y) & =p_{0}^{-1} \circ\left(\left[\lambda\left(p_{0}(X)\right), \lambda\left(p_{0}(Y)\right)\right]-\lambda\left(\left[p_{0}(X), p_{0}(Y)\right]\right)\right) \circ p_{0}, \\
T_{\mathrm{o}}(X, Y) & =p_{0}^{-1} \circ\left(\lambda\left(p_{0}(X)\right) p_{0}(Y)-\lambda\left(p_{0}(Y)\right) p_{0}(X)-\left[p_{0}(X), p_{0}(Y)\right]\right), \\
\mathfrak{I}_{\mathrm{o}}(X) & =p_{0}^{-1} \circ \lambda\left(p_{0}(X)\right) \circ p_{0},
\end{aligned}
$$

para quaisquer $X, Y \in \mathbb{R}^{n}$.

Exemplo 4.4.12 Sejam $\left(M^{n}, g\right)$ uma variedade semi-Riemanniana, onde $g$ tem índice $r$, e $\xi \in \boldsymbol{\Gamma}(T M)$ um campo unitário em $M$, i.e., $g_{x}(\xi(x), \xi(x))=1$, para todo $x \in M$. Consideremos $\mathbb{R}^{n}$ munido da forma bilinear de Minkowski $\langle\cdot, \cdot\rangle$ de índice $r$ e seja $\left\{e_{1}, \ldots, e_{n}\right\}$ a base canônica de $\mathbb{R}^{n}$. Assumimos que existem uma aplicação bilinear $R_{\mathrm{o}}: \mathbb{R}^{n} \times \mathbb{R}^{n} \rightarrow \mathfrak{g l}\left(\mathbb{R}^{n}\right)$ e uma aplicação linear $L_{\mathrm{o}}: \mathbb{R}^{n} \rightarrow \mathbb{R}^{n}$ tais que, para todo $x \in M$ e para toda isometria linear $p: \mathbb{R}^{n} \rightarrow T_{x} M$ com $p\left(e_{1}\right)=\xi(x)$, valem as seguintes condições:

(a) $p^{-1} \circ R_{x}(p \cdot, p \cdot) \circ p=R_{\mathrm{o}}$;

(b) $p \circ L_{\mathrm{o}}=(\nabla \xi)_{x} \circ p$,

onde $\nabla$ é a conexão de Levi-Civita de $(M, g)$. Então, o conjunto

$$
P=\mathrm{FR}^{\mathrm{o}}\left(T M ; e_{1}, \xi\right)
$$

é uma $G$-estrutura em $M$, onde $G=\mathrm{O}_{r}\left(\mathbb{R}^{n} ; e_{1}\right)$ (cf. Exemplo 3.3.13), e a terna $(M, \nabla, P)$ é infinitesimalmente homogênea. De fato, como $\nabla$ é compatível com $g$, o tensor de torção $T$ de $\nabla$ é nulo e a inner torsion $\mathfrak{I}^{P}$ pode ser identificada com a derivada covariante $\nabla \xi$ (cf. Exemplo 3.3.13). Portanto, as condições (a) e (b) acima satisfazem as hipóteses do Lema 4.4.3, e a conlusão segue.

Exemplos de variedades que satisfazem as hipóteses do Exemplo 4.4.12 são as variedades Riemannianas tridimensionais, homogêneas e orientadas, cujo grupo de isometrias tem dimensão 4. Em [10], o autor considera tais variedades, que são fibrações Riemannianas sobre uma forma espacial de dimensão 2; as fibras são geodésicas e existe uma família a 1-parâmetro de translações ao longo das fibras gerada por um campo unitário de Killing $\xi$. Essas variedades são classificadas, a menos de isometrias, pela curvatura $\kappa$ da base da fibração e pela curvatura das fibras $\tau$, onde $\kappa$ e $\tau$ são números reais satisfazendo $\kappa \neq 4 \tau^{2}$. A curvatura das fibras é o número real $\tau$ tal que

$$
\nabla_{X} \xi=\tau X \times \xi(x),
$$


para quaisquer $x \in M$ e $X \in \boldsymbol{\Gamma}(T M)$, onde $\times$ denota o produto vetorial em $T_{x} M$ (determinado pelo produto interno $g_{x}$ e a orientação dada). Neste caso, consideremos a $G$-estrutura

$$
P=\mathrm{FR}_{+}^{\mathrm{o}}\left(T M ; e_{1}, \xi\right),
$$

onde $G=\mathrm{SO}\left(\mathbb{R}^{3} ; e_{1}\right) \simeq \mathrm{SO}\left(\mathbb{R}^{2}\right)$. Mais precisamente, a fibra $P_{x}$ consiste de todas as isometrias lineares $p: \mathbb{R}^{3} \rightarrow T_{x} M$ que preservam orientação tais que $p\left(e_{1}\right)=\xi(x)$. Pelo Exemplo 3.3.13, a inner torsion $\mathfrak{I}_{x}^{P}: T_{x} M \rightarrow$ $\operatorname{sym}\left(T_{x} M\right) \oplus \xi(x)^{\perp}$ é dada por:

$$
\mathfrak{I}_{x}^{P}(v)=(0, \tau v \times \xi(x)),
$$

para quaisquer $x \in M$ e $v \in T_{x} M$. O tensor de curvatura $R$ de $\nabla$ é dado por uma fórmula que depende somente de $\kappa, \tau, \xi$ e a métrica $g$ (ver [10, Proposição 3.1]).

Observação 4.4.13 A classificação das variedades Riemannianas homogêneas, simplesmente conexas, tridimensionais, é bem conhecida (ver [25], [30]). O grupo de isometrias de tais variedades tem dimensão 3, 4 ou 6. Quando a dimensão do grupo de isometrias é 6, tem-se uma forma espacial. Quando a dimensão do grupo de isometrias é 3 , a variedade tem a geometria do grupo de Lie $\mathrm{Sol}_{3}$. O caso de dimensão 4 é considerado em [10]. Quando a curvatura das fibras $\tau$ anula-se (e então $\kappa \neq 0$ ), obtemos uma variedade produto $M^{2}(\kappa) \times \mathbb{R}$, onde $M^{2}(\kappa)$ é uma variedade simplesmente conexa de curvatura seccional constante igual a $\kappa$. Seu grupo de isometrias tem 4 componentes conexas e o campo vertical $\xi$ é simplesmente o vetor correspondente ao fator $\mathbb{R}$. Tais variedades são consideradas em [9]. Quando $\tau \neq 0$, essas variedades são de três tipos: possuem o grupo de isometrias das esferas de Berger para $\kappa>0$, do grupo de Heisenberg $\mathrm{Nil}_{3}$ para $\kappa=0$, e do recobrimento universal $\widehat{\mathrm{PSL}_{2}(\mathbb{R})}$ para $\kappa<0$.

No Capítulo 5 veremos outros exemplos de variedades infinitesimalmente homogêneas. O grupo de Lie $\mathrm{Sol}_{3}$, que no Exemplo 4.4.12 está munido de uma $\mathrm{SO}\left(\mathbb{R}^{2}\right)$-estrutura, será considerado com uma $\overline{\mathrm{Ad}}(H)$-estrutura.

Outros exemplos de variedades infinitesimalmente homogêneas são as variedades de Kähler semi-Riemannianas $\left(M^{n}, g, J\right)$ que têm curvatura holomorfa constante, munidas de uma $\mathrm{U}(n)$-estrutura. Para maiores detalhes, ver [26, Exemplo 6.2]. 


\subsection{Existência de imersões isométricas que preser- vam $G$-estrutura}

Nesta seção discutiremos o teorema, provado em [26], que garante existência de imersões isométricas que preservam $G$-estrutura entre variedades semi-Riemannianas.

Para isso, consideremos os seguintes objetos: uma variedade semi-Riemanniana $(\bar{M}, \bar{g})$ de dimensão $n+k$, onde $\bar{g}$ tem índice $\bar{r}$, uma variedade semi-Riemanniana $(M, g)$ de dimensão $n$, onde $g$ tem índice $r$, um fibrado vetorial $E$ de posto $k$ sobre $M$ munido de uma métrica semi-Riemanniana $g^{E}$ de índice $s$, onde $\bar{r}=r+s$. Consideremos também uma conexão $\nabla^{E}$ em $E$ compatível com $g^{E}$ e uma seção diferenciável simétrica $\alpha$ de $\operatorname{Lin}_{2}(T M ; E)$. Denotemos por $\nabla$ e $\bar{\nabla}$ as conexões de Levi-Civita de $(M, g)$ e $(\bar{M}, \bar{g})$, respectivamente.

Definimos o fibrado vetorial

$$
\widehat{E}=T M \oplus E,
$$

de modo que $\widehat{E}$ é um fibrado vetorial sobre $M$ com fibra típica $\mathbb{R}^{n+k}$. Denotemos por $\widehat{g}$ a métrica semi-Riemanniana de índice $\bar{r}$ em $\widehat{E}$ dada pela soma direta ortogonal de $g$ e $g^{E}$. Consideremos a conexão $\widehat{\nabla}$ em $\widehat{E}$ cujas componentes são $\nabla, \nabla^{E}$ e $\alpha$. Note que $\widehat{\nabla}$ é compatível com $\widehat{g}$.

Suponha a existência de uma imersão isométrica $(f, S)$ de $M$ em $\bar{M}$ (cf. Definição 4.3.1). Definimos um isomorfismo de fibrados vetoriais

$$
L: \widehat{E} \rightarrow T \bar{M}
$$

por

$$
\left.L\right|_{T M}=\mathrm{d} f: T M \rightarrow \mathrm{d} f(T M) \quad \text { e }\left.\quad L\right|_{E}=S: E \rightarrow T M^{\perp} .
$$

O isomorfismo (4.21) é uma isometria entre os fibrados vetoriais $(\widehat{E}, \widehat{g})$ e $(T \bar{M}, \bar{g})$. Reciprocamente, dados uma aplicação diferenciável $f: M \rightarrow \bar{M} \mathrm{e}$ uma isomotria de fibrados vetoriais $L: \widehat{E} \rightarrow T \bar{M}$ então, definindo $S=\left.L\right|_{E}$, o par $(f, S)$ é uma imersão isométrica de $M$ em $\bar{M}$.

Consideremos o espaço Euclidiano $\mathbb{R}^{n+k}$ munido da forma bilinear de Minkowski $\langle\cdot, \cdot\rangle$ de índice $\bar{r}$, e fixemos um subgrupo de Lie $G$ de $\mathrm{O}_{\bar{r}}\left(\mathbb{R}^{n+k}\right)$ com álgebra de Lie $\mathfrak{g} \subset \mathfrak{g l}\left(\mathbb{R}^{n+k}\right)$. Sejam $\widehat{P} \subset \operatorname{FR}^{\mathrm{o}}(\widehat{E})$ e $\bar{P} \subset \mathrm{FR}^{\mathrm{o}}(T \bar{M})$ $G$-estruturas em $\widehat{E}$ e $\bar{M}$, respectivamente.

Definição 4.5.1 Dizemos que uma imersão isométrica $(f, S)$ de $M$ em $\bar{M}$ preserva $G$-estrutura se o isomorfismo de fibrados vetoriais (4.21) preserva 
G-estrutura, ou seja, se

$$
L_{x} \circ p \in \bar{P}_{f(x)},
$$

para quaisquer $x \in M$ e $p \in \widehat{P}_{x}$.

Seja $(f, S)$ uma imersão isométrica de $M$ em $\bar{M}$ que preserva $G$-estrutura. Suponhamos que $(\bar{M}, \bar{\nabla}, \bar{P})$ seja infinitesimalmente homogênea com tensores característicos $\bar{R}_{\mathrm{o}}, \bar{T}_{\mathrm{o}}$ e $\overline{\mathfrak{I}}_{\mathrm{o}}$. Como $\bar{\nabla}$ é a conexão de Levi-Civita de $\bar{M}$, temos $\bar{T}_{\mathrm{o}}=0$ e a aplicação linear $\overline{\mathfrak{I}}_{\mathrm{o}}$ toma valores em $\mathfrak{s o}\left(\mathbb{R}^{n+k}\right) / \mathfrak{g}$. Além disso, como $\widehat{E}_{x}$ é um espaço vetorial com $G$-estrutura, $\widehat{E}_{x}$ herda "versões"

$$
\bar{R}_{\widehat{E}_{x}}: \widehat{E}_{x} \times \widehat{E}_{x} \rightarrow \mathfrak{g l}\left(\widehat{E}_{x}\right) \quad \text { e } \quad \overline{\mathfrak{I}}_{\widehat{E}_{x}}: \widehat{E}_{x} \rightarrow \mathfrak{s o} \mathfrak{o}_{\bar{r}}\left(\widehat{E}_{x}\right) / \mathfrak{g}_{\widehat{E}_{x}}
$$

dos tensores característicos $\bar{R}_{\mathrm{O}}$ e $\overline{\mathfrak{I}}_{\mathrm{o}}$, respectivamente (cf. Observação 4.4.4). Assim, as equações (4.10), (4.11) e (4.12) podem ser escritas, respectivamente, como:

$$
\begin{aligned}
\widehat{g}\left(\bar{R}_{\widehat{E}_{x}}(X, Y) Z, W\right)= & g\left(R_{x}(X, Y) Z, W\right)+g^{\perp}\left(\alpha_{x}^{f}(X, Z), \alpha_{x}^{f}(Y, W)\right) \\
& -g^{\perp}\left(\alpha_{x}^{f}(X, W), \alpha_{x}^{f}(Y, Z)\right) \\
\widehat{g}\left(\bar{R}_{\widehat{E}_{x}}(X, Y) Z, \eta\right)=g^{\perp}\left(\left(\nabla_{X}^{\perp} \alpha^{f}\right)(Y, Z), \eta\right)-g^{\perp}\left(\left(\nabla_{Y}^{\perp} \alpha^{f}\right)(X, Z), \eta\right), & \\
\widehat{g}\left(\bar{R}_{\widehat{E}_{x}}(X, Y) \eta, \xi\right)= & g^{\perp}\left(R_{x}^{\perp}(X, Y) \eta, \xi\right)+g(A(X, \eta), A(Y, \xi)) \\
& -g(A(X, \xi), A(Y, \eta)) .
\end{aligned}
$$

Com a notação e os dados fixados acima, temos o seguinte:

Teorema 4.5.2 (i) Assuma que $(\bar{M}, \bar{\nabla}, \bar{P})$ é infinitesimalmente homogênea com tensores característicos $\bar{R}_{\mathrm{o}}, \bar{T}_{\mathrm{o}}$ e $\overline{\mathfrak{I}}_{\mathrm{O}}$, e que sejam válidas as seguintes equações:

$$
\begin{aligned}
& \widehat{g}\left(\bar{R}_{\widehat{E}_{x}}(X, Y) Z, W\right)= g\left(R_{x}(X, Y) Z, W\right)+g^{E}(\alpha(X, Z), \alpha(Y, W)) \\
&-g^{E}(\alpha(X, W), \alpha(Y, Z)) \\
& \widehat{g}\left(\bar{R}_{\widehat{E}_{x}}(X, Y) Z, \eta\right)=g^{E}\left(\left(\nabla_{X}^{E} \alpha\right)(Y, Z), \eta\right)-g^{E}\left(\left(\nabla_{Y}^{E} \alpha\right)(X, Z), \eta\right), \\
& \widehat{g}\left(\bar{R}_{\widehat{E}_{x}}(X, Y) \eta, \xi\right)=g^{E}\left(R_{x}^{E}(X, Y) \eta, \xi\right)+g(A(X, \eta), A(Y, \xi)) \\
&- g(A(X, \xi), A(Y, \eta)) \\
& \overline{\mathfrak{I}}_{\widehat{E}_{x}}(X)=\mathfrak{I}_{x}^{\widehat{P}}(X),
\end{aligned}
$$


para quaisquer $x \in M, X, Y, Z, W \in T_{x} M$ e $\eta, \xi \in E_{x}$, onde $A$ é definido por

$$
g^{E}(\alpha(X, Y), \eta)=-g(A(X, \eta), Y) .
$$

Então, para quaisquer $x_{0} \in M$ e $y_{0} \in \bar{M}$, e para toda aplicação linear $\sigma_{0}: \widehat{E}_{x_{0}} \rightarrow T_{y_{0}} \bar{M}$ que preserva $G$-estrutura, existe uma imersão isométrica $(f, S)$, cujo dominio é uma vizinhança $U$ de $x_{0}$ em $M$, tal que $f\left(x_{0}\right)=y_{0}$ e $\sigma_{0}=L_{x_{0}}$, onde $L$ é como em (4.21). Além disso, se $M$ é simplesmente conexa e $(\bar{M}, \bar{\nabla})$ é geodesicamente completa então existe uma única imersão isométrica global $(f, S)$ tal que $f\left(x_{0}\right)=y_{0}$ e $\sigma_{0}=L_{x_{0}}$.

(ii) Assuma que $M$ é conexa e sejam $\left(f^{1}, S^{1}\right),\left(f^{2}, S^{2}\right)$ imersões isométricas de $M$ em $\bar{M}$ com os mesmos dados prescritos $\nabla^{E}, \alpha, g^{E}$. Suponha que exista um ponto $x_{0} \in M$ tal que

$$
f^{1}\left(x_{0}\right)=f^{2}\left(x_{0}\right), \quad \mathrm{d} f_{x_{0}}^{1}=\mathrm{d} f_{x_{0}}^{2}, \quad S_{x_{0}}^{1}=S_{x_{0}}^{2} .
$$

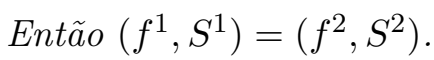

Observação 4.5.3 O Teorema 4.5.2 vale em um contexto mais geral, no contexto de variedades afins. Mais precisamente, se $(M, \nabla)$ e $(\bar{M}, \bar{\nabla})$ são variedades afins munidas de $G$-estruturas e valem as hipóteses do Teorema 4.5.2 (com as devidas adaptações), podemos obter o mesmo resultado para variedades afins (ver [26, Teorema 7.4)].

Veremos a seguir alguns exemplos explícitos de aplicações do Teorema 4.5.2.

Exemplo 4.5.4 Consideremos o caso em que a variedade semi-Riemanniana $(\bar{M}, \bar{g})$ tem curvatura seccional constante igual a $c$. Sejam $G=\mathrm{O}_{\bar{r}}\left(\mathbb{R}^{n+k}\right)$ e $\bar{P}=\mathrm{FR}^{\mathrm{o}}(T \bar{M})$, de modo que $(\bar{M}, \bar{\nabla}, \bar{P})$ é infinitesimalmente homogênea (cf. Exemplo 4.4.8). Considerando $\widehat{P}=\mathrm{FR}^{\mathrm{o}}(\widehat{E})$, temos $\mathfrak{I}^{\widehat{P}}=0$ e $\overline{\mathfrak{I}}_{\widehat{E}}=0$ pois as conexões $\widehat{\nabla}$ e $\bar{\nabla}$ são compatíveis com as métricas semi-Riemannianas $\widehat{g}$ e $\bar{g}$, respectivamente (cf. Exemplo 3.3.9). Assim, a hipótese (4.28) do Teorema 4.5.2 é automaticamente satisfeita. Do Exemplo 4.4.8, o lado esquerdo da equação de Gauss torna-se:

$$
c\left(g_{x}(X, Z) g_{x}(Y, W)-g_{x}(Y, Z) g_{x}(X, W)\right),
$$

para quaisquer $x \in M$ e $X, Y, Z, W \in T_{x} M$. O lado esquerdo das equações de Codazzi e Ricci são nulos. Assim, neste caso, o Teorema 4.5.2 reproduz o clássico Teorema Fundamental de Imersões Isométricas (ver, por exemplo, 
[7], [33]). De forma mais explícita, para quaisquer $x_{0} \in M$ e $y_{0} \in \bar{M}$, e para toda isometria linear $\sigma_{0}: \widehat{E}_{x_{0}} \rightarrow T_{y_{0}} \bar{M}$, existe uma imersão isométrica $(f, S)$ que preserva $G$-estrutura, cujo domínio é uma vizinhança $U$ de $x_{0}$ em $M$, tal que $f\left(x_{0}\right)=y_{0}$ e $\sigma_{0}=\mathrm{d} f_{x_{0}} \oplus S_{x_{0}}$.

Exemplo 4.5.5 Sejam $\left(M_{1}^{n_{1}}, g^{1}\right),\left(M_{2}^{n_{2}}, g^{2}\right)$ variedades semi-Riemannianas tais que o índice de $g^{i}$ é $r_{i}$ e a curvatura seccional de $\left(M_{i}, g^{i}\right)$ é constante e igual a $c_{i}, i=1,2$. Assuma que $(\bar{M}, \bar{g})$ é o produto cartesiano ortogonal de $\left(M_{1}^{n_{1}}, g^{1}\right)$ e $\left(M_{2}^{n_{2}}, g^{2}\right)$, de modo que $n+k=n_{1}+n_{2}$ e $\bar{r}=r_{1}+r_{2}$. Seja $\mathbb{R}^{n+k}=\mathbb{R}^{n_{1}} \oplus \mathbb{R}^{n_{2}}$ munido com a soma ortogonal das formas bilineares de Minkowski de índices $r_{1}$ e $r_{2}$. Então, a $G$-estrutura

$$
\bar{P}=\mathrm{FR}^{\mathrm{o}}\left(T \bar{M} ; \mathbb{R}^{n_{1}} \oplus\{0\}^{n_{2}}, \operatorname{pr}_{1}^{*}\left(T M_{1}\right)\right),
$$

torna $(\bar{M}, \bar{\nabla}, \bar{P})$ infinitesimalmente homogênea (cf. Exemplo 4.4.9), onde

$$
G=\mathrm{O}_{\bar{r}}\left(\mathbb{R}^{n+k} ; \mathbb{R}^{n_{1}} \oplus\{0\}^{n_{2}}\right) \cong \mathrm{O}_{r_{1}}\left(\mathbb{R}^{n_{1}}\right) \times \mathrm{O}_{r_{2}}\left(\mathbb{R}^{n_{2}}\right)
$$

e $\operatorname{pr}_{1}: \bar{M} \rightarrow M_{1}$ denota a projeção sobre o primeiro fator. Seja $F$ um subfibrado vetorial de $\widehat{E}$, com $\operatorname{rank}(F)=n_{1}$ e $\widehat{E}=F \oplus F^{\perp}$. Consideremos

$$
\widehat{P}=\mathrm{FR}^{\mathrm{o}}\left(\widehat{E} ; \mathbb{R}^{n_{1}} \oplus\{0\}^{n_{2}}, F\right) .
$$

Assumindo que $\widehat{P}_{x} \neq \emptyset$, para todo $x \in M$, segue do Exemplo 3.3.11 que $\widehat{P}$ é uma $G$-estrutura em $\widehat{E}$. Como $\bar{\nabla}$ é compatível com $\bar{g}$, temos $\mathfrak{I}^{\bar{P}}=0$ e, portanto, $\overline{\mathfrak{I}}_{\widehat{E}}=0$. Assim, a hipótese (4.28) do Teorema 4.5.2 significa que $\mathfrak{I}^{\widehat{P}}=0$, ou seja, a derivada covariante $\widehat{\nabla}$ de seções de $F$ são seções de $F$ (cf. Exemplo 3.3.11). Denotemos por $\pi^{F}: \widehat{E} \rightarrow F$ e $\pi^{F^{\perp}}: \widehat{E} \rightarrow F^{\perp}$ as projeções correspondentes à decomposição em soma direta $\widehat{E}=F \oplus F^{\perp}$. O lado esquerdo das equações (4.25), (4.26) e (4.27) torna-se, respectivamente:

$$
\begin{gathered}
\left.c_{2}\left[\widehat{g}\left(\pi^{F}(X), \pi^{F}(Z)\right) \widehat{g}\left(\pi^{F}(Y) \pi^{F}(W)\right)-\widehat{g}\left(\pi^{F}(X), \pi^{F}(W)\right) \widehat{g}\left(\pi^{F}(Y), \pi^{F}(Z)\right)\right]\right] \\
+c_{1}\left[\widehat{g}\left(\pi^{F^{\perp}}(X), \pi^{F^{\perp}}(Z)\right) \widehat{g}\left(\pi^{F^{\perp}}(Y) \pi^{F^{\perp}}(W)\right)\right. \\
\left.-\widehat{g}\left(\pi^{F^{\perp}}(X), \pi^{F^{\perp}}(W)\right) \widehat{g}\left(\pi^{F^{\perp}}(Y), \pi^{F^{\perp}}(Z)\right)\right] \\
c_{2}\left[\widehat{g}\left(\pi^{F}(X), \pi^{F}(Z)\right) \widehat{g}\left(\pi^{F}(Y) \pi^{F}(\eta)\right)-\widehat{g}\left(\pi^{F}(X), \pi^{F}(\eta)\right) \widehat{g}\left(\pi^{F}(Y), \pi^{F}(Z)\right)\right] \\
+c_{1}\left[\widehat{g}\left(\pi^{F^{\perp}}(X), \pi^{F^{\perp}}(Z)\right) \widehat{g}\left(\pi^{F^{\perp}}(Y) \pi^{F^{\perp}}(\eta)\right)\right. \\
\left.-\widehat{g}\left(\pi^{F^{\perp}}(X), \pi^{F^{\perp}}(\eta)\right) \widehat{g}\left(\pi^{F^{\perp}}(Y), \pi^{F^{\perp}}(Z)\right)\right]
\end{gathered}
$$




$$
\begin{array}{r}
c_{2}\left[\widehat{g}\left(\pi^{F}(X), \pi^{F}(\eta)\right) \widehat{g}\left(\pi^{F}(Y) \pi^{F}(\xi)\right)-\widehat{g}\left(\pi^{F}(X), \pi^{F}(\xi)\right) \widehat{g}\left(\pi^{F}(Y), \pi^{F}(\eta)\right)\right] \\
+c_{1}\left[\widehat{g}\left(\pi^{F^{\perp}}(X), \pi^{F^{\perp}}(\eta)\right) \widehat{g}\left(\pi^{F^{\perp}}(Y) \pi^{F^{\perp}}(\xi)\right)\right. \\
\left.\quad-\widehat{g}\left(\pi^{F^{\perp}}(X), \pi^{F^{\perp}}(\xi)\right) \widehat{g}\left(\pi^{F^{\perp}}(Y), \pi^{F^{\perp}}(\eta)\right)\right]
\end{array}
$$

A tese do Teorema 4.5.2 torna-se, então: para quaisquer $x_{0} \in M$ e $y_{0}=$ $\left(y_{0}^{1}, y_{0}^{2}\right) \in \bar{M}$, e para toda isometria linear $\sigma_{0}: \widehat{E}_{x_{0}} \rightarrow T_{y_{0}} \bar{M}$ que preserva $G$-estrutura, i.e., que satisfaz

$$
\sigma_{0}\left(F_{x_{0}}\right)=T_{y_{0}^{1}} M_{1} \oplus\{0\}
$$

existe uma imersão isométrica $(f, S)$ que preserva $G$-estrutura, cujo domínio é uma vizinhança $U$ de $x_{0}$ em $M$, tal que

$$
f\left(x_{0}\right)=y_{0} \quad \text { e } \quad L_{x_{0}}=\sigma_{0} .
$$

Exemplo 4.5.6 Um caso particular do Exemplo 4.5.5 é quando a variedade Riemanniana $\bar{M}$ é um produto ortogonal da forma $\bar{M}=Q_{c}^{n} \times \mathbb{R}$, onde $Q_{c}^{n}$ é uma variedade Riemanniana de curvatura seccional constante igual a c. Consideremos $\mathbb{R}^{n+1}$ munido do produto interno Euclidiano $\langle\cdot, \cdot\rangle$ e seja $\left\{e_{1}, \ldots, e_{n+1}\right\}$ a base canônica de $\mathbb{R}^{n+1}$. Então, a $G$-estrutura

$$
\bar{P}=\operatorname{FR}^{\mathrm{o}}\left(T \bar{M} ;\{0\}^{n} \oplus \mathbb{R}, \operatorname{pr}_{2}^{*}(T \mathbb{R})\right)
$$

torna $(\bar{M}, \bar{\nabla}, \bar{P})$ infinitesimalmente homogênea, onde

$$
G=\mathrm{O}\left(\mathbb{R}^{n+1} ;\{0\}^{n} \oplus \mathbb{R}\right) \simeq \mathrm{O}\left(\mathbb{R}^{n}\right) \times \mathrm{O}(\mathbb{R})
$$

e $\operatorname{pr}_{2}: \bar{M} \rightarrow \mathbb{R}$ denota a projeção sobre o segundo fator. Assumimos que $E=M \times \mathbb{R}$ é o fibrado vetorial trivial sobre $M$, e consideremos $T \in \boldsymbol{\Gamma}(T M)$ e $\nu \in \mathrm{C}^{\infty}(M)$ tais que $\|T\|^{2}+\nu^{2}=1$. O par $(T, \nu)$ é uma seção diferenciável de $\widehat{E}$ e, portanto, o subfibrado vetorial $F$ de $\widehat{E}$, definido por

$$
F_{x}=\operatorname{span}\{(T(x), \nu(x))\},
$$

para todo $x \in M$, é tal que $\widehat{E}=F \oplus F^{\perp}$. Além disso,

$$
\widehat{P}=\operatorname{FR}^{\mathrm{o}}\left(\widehat{E} ;\{0\}^{n} \oplus \mathbb{R}, F\right)
$$

é uma $G$-estrutura em $\widehat{E}$. Mais precisamente, dado $x \in M$, a fibra $\widehat{P}_{x}$ é o conjunto de todas as isometrias lineares $p: \mathbb{R}^{n+1} \rightarrow \widehat{E}_{x}$ tais que $p\left(e_{n+1}\right)=$ $(T(x), \nu(x))$. A $G$-estrutura $\widehat{P}$ será denotada simplesmente por

$$
\widehat{P}=\operatorname{FR}^{\mathrm{o}}\left(\widehat{E} ; e_{n+1},(T, \nu)\right) .
$$


Fixado uma seção unitária $\epsilon \in \boldsymbol{\Gamma}(E)$, temos um operador $A: \boldsymbol{\Gamma}(T M) \rightarrow$ $\boldsymbol{\Gamma}(T M)$ associado a $\epsilon$ dado por

$$
g(A X, Y)=g^{E}(\alpha(X, Y), \epsilon)
$$

para quaisquer $X, Y \in \boldsymbol{\Gamma}(T M)$. Neste caso, as equações de Gauss e Codazzi podem ser escritas como

$$
R(X, Y)+A X \wedge A Y=c(X \wedge Y+g(X, T) Y \wedge T-g(Y, T) X \wedge T)
$$

e

$$
\left(\nabla_{X} A\right) Y-\left(\nabla_{Y} A\right) X=c \nu(Y \wedge X) T
$$

respectivamente, para quaisquer $X, Y \in \Gamma(T M)$. De fato, neste caso, a equação de Gauss (4.25) pode ser escrita como:

$$
\pi^{M}\left(\bar{R}_{\widehat{E}}(X, Y) Z\right)=(A X \wedge A Y) Z+R(X, Y) Z,
$$

para quaisquer $X, Y, Z \in \boldsymbol{\Gamma}(T M)$, onde $\pi^{M}: \widehat{E} \rightarrow T M$ denota a projeção canônica (cf. Exemplo 4.3.5). Pelo Exemplo 4.5.5, o lado esquerdo de (4.31) torna-se:

$$
c\left[\widehat{g}\left(\pi^{F^{\perp}}(X), \pi^{F^{\perp}}(Z)\right) \pi^{F^{\perp}}(Y)-\widehat{g}\left(\pi^{F^{\perp}}(Y), \pi^{F^{\perp}}(Z)\right) \pi^{F^{\perp}}(X)\right] .
$$

Por outro lado, para todo $X \in \boldsymbol{\Gamma}(T M)$, temos:

$$
\pi^{F^{\perp}}(X)=X-g(X, T) \widehat{T} .
$$

Substituindo em (4.32) o valor de $\pi^{F^{\perp}}(X)$ e expressões similares para $\pi^{F^{\perp}}(Y)$ e $\pi^{F^{\perp}}(Z)$, o lado esquerdo de (4.31) torna-se:

$$
c[(X \wedge Y) Z+g(X, T)(Y \wedge T) Z-g(Y, T)(X \wedge T) Z] .
$$

Isso fornce a equação (4.29). A equação de Codazzi (4.26) pode ser escrita, neste caso, como:

$$
\pi^{M}\left(\bar{R}_{\widehat{E}}(X, Y) N\right)=\left(\nabla_{X} A\right) Y-\left(\nabla_{Y} A\right) X,
$$

para quaisquer $X, Y \in \boldsymbol{\Gamma}(T M)$ (cf. Exemplo 4.3.5). Pelo Exemplo 4.5.5, o lado esquerdo de (4.33) torna-se:

$$
c\left[\widehat{g}\left(\pi^{F^{\perp}}(X), \pi^{F^{\perp}}(N)\right) \pi^{F^{\perp}(Y)}-\widehat{g}\left(\pi^{F^{\perp}}(Y), \pi^{F^{\perp}}(N)\right) \pi^{F^{\perp}}(X)\right] .
$$


Finalmente, temos $\pi^{F^{\perp}}(N)=N-\nu \widehat{T}$. Substituindo o valor de $\pi^{F^{\perp}}(N)$ em (4.34) e expressões similares para $\pi^{F^{\perp}}(X)$ e $\pi^{F^{\perp}}(Y)$, o lado esquerdo de (4.33) torna-se

$$
c \nu(X \wedge Y) T,
$$

fornecendo, assim, a equação (4.30). A hipótese (4.28) do Teorema 4.5.2 é análoga a do Exemplo 4.5.5. Neste caso, o Teorema 4.5.2 reproduz o teorema de imersão isométrica em $Q_{c}^{n} \times \mathbb{R}$ de [9].

Exemplo 4.5.7 Consideremos o caso em que $\bar{M}=K$ é um grupo de Lie, com álgebra de Lie $\mathfrak{k}$, e $\bar{g}$ é uma métrica semi-Riemanniana invariante à esquerda em $\bar{M}$. Fixemos uma isometria linear $p_{0}: \mathbb{R}^{n+k} \rightarrow \mathfrak{k}$ e consideremos a seção global diferenciável $s: \bar{M} \rightarrow \mathrm{FR}^{\mathrm{o}}(T \bar{M})$ definida por $s(g)=\mathrm{d} L_{g}(1) \circ$ $p_{0}$, para todo $g \in K$. Então, a $G$-estrutura

$$
\bar{P}=s(\bar{M})
$$

torna $(\bar{M}, \bar{\nabla}, \bar{P})$ infinitesimalmente homogênea, onde $G=\left\{\operatorname{Id}_{\mathbb{R}^{n+k}}\right\}$ (cf. Exemplo 4.4.11). A inner torsion $\mathfrak{I}^{P}$, no elemento identidade $1 \in K$, coincide com a aplicação linear $\lambda: \mathfrak{k} \rightarrow \mathfrak{g l}(\mathfrak{k})$, onde $\lambda$ é dada por

$$
\left.\bar{g}(\lambda(X) Y, Z)=\frac{1}{2}(\bar{g}([X, Y], Z])-\bar{g}([X, Z], Y)-\bar{g}([Y, Z], X)\right),
$$

para quaisquer $X, Y, Z \in \mathfrak{k}$ (cf. Teorema 2.6.7). Seja $\hat{s}: M \rightarrow \mathrm{FR}^{\mathrm{o}}(\widehat{E})$ uma seção global diferenciável de $\widehat{E}$, de modo que

$$
\widehat{P}=\hat{s}(M)
$$

é uma $G$-estrutura em $\widehat{E}$. A hipótese (4.28) do Teorema 4.5.2 significa que, para todo $x \in M$, a igualdade

$$
\widehat{\Gamma}_{x}=\operatorname{Ad}_{\hat{s}(x)} \circ \overline{\mathfrak{I}}_{\mathrm{O}} \circ \hat{s}(x)^{-1}
$$

é satisfeita, onde $\widehat{\Gamma}$ denota o tensor de Christoffel de $\widehat{\nabla}$ em relação a $\hat{s}$ e $\overline{\mathfrak{I}}_{\mathrm{O}}$ é o tensor característico correspondente à inner torsion $\mathfrak{I}^{\bar{P}}$ (cf. Exemplo 4.4.11). A tese do Teorema 4.5.2 torna-se: para quaisquer $x_{0} \in M$ e $y_{0} \in \bar{M}$, existe uma imersão isométrica $(f, S)$, cujo domínio é uma vizinhança $U$ de $x_{0}$ em $M$, tal que $f\left(x_{0}\right)=y_{0}$ e

$$
\left(\mathrm{d} f_{x} \oplus S_{x}\right) \circ \hat{s}(x)=s(f(x))
$$

para todo $x \in U$. 
Exemplo 4.5.8 Assumimos que a variedade semi-Riemanniana $(\bar{M}, \bar{g})$ está munida de um campo vetorial unitário $\xi \in \Gamma(T \bar{M})$ tal que as condições descritas no Exemplo 4.4.12 estejam satisfeitas. A $G$-estrutura

$$
\bar{P}=\mathrm{FR}^{\mathrm{o}}\left(T \bar{M} ; e_{1}, \xi\right)
$$

torna $(\bar{M}, \bar{\nabla}, \bar{P})$ infinitesimalmente homogênea, onde $G=\mathrm{O}_{\bar{r}}\left(\mathbb{R}^{n+k} ; e_{1}\right)$ (cf. Exemplo 4.4.12). Consideremos uma seção $\epsilon \in \boldsymbol{\Gamma}(\widehat{E})$ ( $\epsilon$ é determinada por um campo vetorial diferenciável em $M$ e uma seção global diferenciável de $E)$ tal que $\hat{g}(\epsilon, \epsilon) \equiv 1$. Seja

$$
\widehat{P}=\mathrm{FR}^{\mathrm{o}}\left(\widehat{E} ; e_{1}, \epsilon\right)
$$

de modo que $\widehat{P}$ é uma $G$-estrutura em $\widehat{E}$. A hipótese (4.28) do Teorema 4.5.2 significa que, para todo $x \in M$ e para todo $p \in \widehat{P}_{x}$, tem-se

$$
\left.p \circ L_{\mathrm{o}}\right|_{p^{-1}\left(T_{x} M\right)}=\left.(\widehat{\nabla} \epsilon)_{x} \circ p\right|_{p^{-1}\left(T_{x} M\right)} .
$$

A tese do Teorema 4.5.2 torna-se: para quaisquer $x_{0} \in M$ e $y_{0} \in \bar{M}$, e para toda isometria linear $\sigma_{0}: \widehat{E}_{x_{0}} \rightarrow T_{y_{0}} \bar{M}$ com $\sigma_{0}\left(\epsilon\left(x_{0}\right)\right)=\xi\left(y_{0}\right)$, existe uma imersão isométrica $(f, S)$, cujo domínio é uma vizinhança $U$ de $x_{0}$ em $M$, tal que $f\left(x_{0}\right)=y_{0}$ e

$$
\left(\mathrm{d} f_{x} \oplus S_{x}\right)=\xi(f(x)),
$$

para todo $x \in U$. No caso particular em que $(\bar{M}, \bar{g})$ é uma variedade Riemanniana homogênea orientável de dimensão 3 , cujo grupo de isometrias tem dimensão 4, o Teorema 4.5.2 reproduz o teorema de existência de imersões isométricas considerado em [10]. Neste caso, as $G$-estruturas $\bar{P}$ e $\widehat{P}$ são dadas por

$$
\bar{P}=\mathrm{FR}_{+}^{\mathrm{o}}\left(T \bar{M} ; e_{1}, \xi\right)
$$

e

$$
\widehat{P}=\mathrm{FR}_{+}^{\mathrm{o}}\left(\widehat{E} ; e_{1},(T, \nu)\right),
$$

onde $T \in \boldsymbol{\Gamma}(T M)$ e $\nu \in \mathrm{C}^{\infty}(M)$ são tais que $\|T\|^{2}+\nu^{2}=1$.

Outro exemplo, que pode ser visto como aplicação do Teorema 4.5.2, é o caso em que $(\bar{M}, \bar{g})$ é uma variedade semi-Riemanniana de Kähler com curvatura seccional holomorfa constante. Para maiores detalhes, ver [26, Exemplo 8.2]. 


\section{Capítulo 5}

\section{Imersões Isométricas em 3-Variedades Lorentzianas}

\subsection{Introdução}

A teoria de classificação das 3-variedades Riemannianas foi estabelecida por Thurston (ver [34]), onde o autor afirma que, no caso de dimensão 3, existem apenas oito geometrias Riemannianas maximais. Em [30], Scott descreve cada uma dessas oito geometrias e suas propriedades básicas, dando uma descrição completa das variedades fechadas tridimensionais que admitem uma estrutura geométrica, além daquelas que admitem uma estrutura hiperbólica.

O caso Lorentziano, em analogia ao caso Riemanniano, tem sido estudado recentemente. Em [11], os autores provam que existem somente quatro geometrias Lorentzianas maximais.

\subsubsection{O Teorema de Classificação de Thurston}

Dizemos que uma variedade diferenciável $M^{n}$ admite uma estrutura geométrica se existe uma métrica localmente homogênea completa em $M$. Isso significa que o recobrimento universal $X$ de $M$ possui uma métrica homogênea completa (ver Seção 3 de [31]), de modo que o grupo de isometrias de $X$ age transitivamente. Dizemos também que $M$ admite uma estrutura geométrica modelada em $X$.

Uma geometria é um par $(G, X)$, onde $G$ é um grupo que age transitivamente sobre uma variedade diferenciável $X$, com subgrupos de isotropia compacto. Dizemos que duas geometrias $(G, X)$ e $(H, Y)$ são equivalentes 
se existem um homomorfismo $\phi_{\mathrm{o}}: G \rightarrow H$ e um difeomorfismo $\varphi: X \rightarrow Y$ tais que $\varphi(g \cdot x)=\phi_{\mathrm{o}}(g) \cdot \varphi(x)$, para quaisquer $g \in G$ e $x \in X$. Segue, em particular, que $G$ e $H$ são grupos de Lie isomorfos.

Dado uma geometria $(G, X)$, existe uma geometria natural $(\widetilde{G}, \widetilde{X})$ associada, onde $\widetilde{X}$ denota o recobrimento universal de $X$ e $\widetilde{G}$ consiste de todos os difeomorfismos de $\widetilde{X}$ que são levantamentos dos elementos de $G$. Assim, não há perda de generalidade em considerar somente geometrias $(G, X)$ onde $X$ é simplesmente conexo. Uma restrição final no caso de dimensão 3 é que existe um subgrupo $H$ de $G$ que age em $X$ como grupo de recobrimento e tem quociente compacto. Dizemos, neste caso, que a geometria admite um quociente compacto.

Teorema 5.1.1 (Thurston) Qualquer geometria tridimensional maximal, simplesmente conexa e que admite um quociente compacto é equivalente a uma das geometrias ( $\operatorname{Iso}(X), X)$, onde $X$ é uma das seguintes variedades: $\mathbb{R}^{3}, \mathbb{H}^{3}, \mathbb{S}^{3}, \mathbb{S}^{2} \times \mathbb{R}, \mathbb{H}^{2} \times \mathbb{R}, \widetilde{\mathrm{SL}_{2}(\mathbb{R})}$, Nil ou Sol.

Para uma apresentação da prova de Thurston, ver [30, Teorema 5.1]. Além disso, se $M$ é uma variedade fechada tridimensional que admite uma estrutura geométrica modelada em uma das oito geometrias do Teorema 5.1.1, então a geometria envolvida é única (ver [30, Teorema 5.2]).

\subsubsection{O Teorema de Classificação Lorentziana}

Consideremos um espaço homogêneo simplesmente conexo $G / H$, onde $G$ é um grupo de Lie e $H$ é um subgrupo fechado de $G$. Dizemos que a geometria $(G, G / H)$ é Lorentziana se a ação canônica de $G$ sobre $G / H$ preserva alguma métrica Lorentziana (cf. Definição 1.6.1) ou, de forma equivalente, se a ação adjunta de $H$ sobre $\mathfrak{g} / \mathfrak{h}$ preserva uma forma bilinear simétrica nãodegenerada de índice 1 (cf. Lema 1.6.7).

Dizemos que uma variedade diferenciável $M$ admite uma $(G, G / H)$-estrutura se existe um atlas

$$
\left\{\phi_{i}: U_{i} \rightarrow \phi_{i}\left(U_{i}\right) \subset G / H\right\}
$$

de $M$, onde as aplicações de transição $\gamma_{i j}$, que satisfazem $\gamma_{i j} \circ \phi_{i}=\phi_{j}$, são dadas por restrição dos elementos de $G$. Mais precisamente, as aplicações $\gamma_{i j}$ são determinadas por aplicações localmente constantes de $U_{i} \cap U_{j}$ em $G$. Dizemos também, neste caso, que $M$ é localmente modelada sobre o espaço homogêneo $G / H$ ou, também, que $M$ é uma $(G, G / H)$-variedade. 
Uma geometria Lorentziana $(G, G / H)$ é chamada maximal se o grupo de Lie $G$ é de dimensão máxima sobre todos os grupos de Lie que agem transitivamente sobre $G / H$ e preservam uma métrica Lorentziana.

Uma $(G, G / H)$-estrutura sobre $M$ define uma aplicação desenvolvível

$$
D: \widetilde{M} \rightarrow G / H
$$

que é um difeomorfismo local entre o recobrimento unviversal $\widetilde{M}$ de $M$ e o espaço modelo $G / H^{1}$. Além disso, $D$ é única a menos de composição com elementos de $G$.

Uma $(G, G / H)$-variedade $M$ é dita ser completa se sua aplicação desenvolvível (5.1) é um difeomorfismo. Por exemplo, seja $(G, G / H)$ uma geometria tal que, para todo $x \in G / H$, o subgrupo de isotropia $G_{x}$ é compacto. Então, toda $(G, G / H)$-variedade fechada $M$ é completa (ver [34, Proposição 3.6]). Como $G / H$ é simplesmente conexo, qualquer $(G, G / H)$ variedade completa $M$ pode ser reconstruída da imagem $\Gamma=H\left(\pi_{1}(M)\right)$ da holonomia, como o espaço quociente $(G / H) / \Gamma$.

Quando a ação de $G$ sobre $G / H$ preserva uma métrica Lorentziana ou, mais geralmente, uma conexão linear, temos a noção de completude geodésica. A completude geodésica é mais forte que a completude acima definida. De fato, seja $M$ uma $(G, G / H)$-variedade tal que a ação de $G$ sobre $G / H$ preserva uma conexão linear $\nabla$. Se a conexão induzida sobre $M$ por $\nabla$ é geodesicamente completa então $M$ é completa (ver [11, Lema 2.2]).

O Lema seguinte garante a unicidade do modelo $G / H$. Mais precisamente,

Lema 5.1.2 Toda variedade Lorentziana $M$ de dimensão 3 , compacta e localmente homogênea, é localmente modelada sobre uma única geometria maximal $(G, G / H)$, no caso em que $G$ é conexo. A dimensão de $G$ é $\leq 6$ e a igualdade ocorre se, e somente se, $M$ é de curvatura seccional constante. Além $\operatorname{disso}, \operatorname{dim}(G) \neq 5$ (isso exclui a possibilidade $\operatorname{de} \operatorname{dim}(H)=2$ ).

Disso decorre que as geometrias Lorentzianas de curvatura seccional constante são maximais, pois elas realizam a dimensão máxima do grupo de isometrias. Tais geometrias são a gemetria de Minkowski (curvatura nula), a geometria de Sitter (curvatura positiva) e a geometria anti-de Sitter (curvatura negativa). Para a prova do Lema 5.1.2, ver a demonstração da Proposição 3.1 de [11].

\footnotetext{
${ }^{1}$ Para maiores detalhes sobre a aplicação desenvolvível (5.1), ver Seção 3.5 de [34].
} 
O teorena de classificação das 3-variedades Lorentzianas, cuja demonstração está ao longo de [11], é o seguinte:

Teorema 5.1.3 Seja $M$ uma variedade Lorentziana compacta conexa tridimensional, localmente modelada sobre uma geometria Lorentziana $(G, G / H)$. Então, o modelo $G / H$ é isométrico a um dos seguintes grupos de Lie, munidos de uma métrica Lorentziana invariante à esquerda: $\mathbb{R}^{3}, \widetilde{\mathrm{SL}_{2}(\mathbb{R})}$, Nil ou Sol. Se a geometria $(G, G / H)$ é maximal, então $(G, G / H)$ é uma das seguintes geometrias: Minkowski, anti-de Sitter, Heisenberg-Lorentz ou SolLorentz. Além disso, a geometria $(G, G / H)$ é completa, e a variedade $M$ é geodesicamente completa, exceto no caso em que ela é localmente modelada sobre a geometria Sol-Lorentz.

Um modelo da geometria de Minkowski é o espaço Euclidiano $\mathbb{R}^{3}$ munido da métrica Lorentziana $g=d x^{2}+d y^{2}-d z^{2}$. O grupo $G$ dessa geometria é $G=\mathrm{O}(2,1) \ltimes \mathbb{R}^{3}$, com a ação canônica de $\mathrm{O}(2,1)$ sobre $\mathbb{R}^{3}$. A isotropia $H$ da geometria de Minkowski é $\mathrm{O}(2,1)$.

Um modelo da geometria anti-de Sitter é o recobrimento universal $\widetilde{\mathrm{SL}_{2}(\mathbb{R})}$ de $\mathrm{SL}_{2}(\mathbb{R})$, munido da métrica Lorentziana invariante à esquerda que coincide, no elemento identidade, com a forma de Killing sobre a álgebra de Lie $\mathfrak{s l}_{2}(\mathbb{R})$ de $\mathrm{SL}_{2}(\mathbb{R})$. O grupo $G$ dessa geometria é $G=\widetilde{\mathrm{SL}_{2}(\mathbb{R})} \times \widetilde{\mathrm{SL}_{2}(\mathbb{R})}$.

A geometria Heisenberg-Lorentz tem como modelo o grupo de Heisenberg Nil munido da métrica Lorentziana invariante à esquerda

$$
g=-\mathrm{d} x^{2}+\mathrm{d} y^{2}+(\mathrm{d} z-x \mathrm{~d} y)^{2} .
$$

O grupo $G$ dessa geometria é $G=\mathrm{Iso}(\mathrm{Nil})$.

A geometria Sol-Lorentz é obtida a partir do grupo de Lie Sol munido de uma métrica Lorentziana invariante à esquerda definida na álgebra de Lie sol, que é gerada por $T, X, Z$. A métrica é definida exigindo-se que $T$ e $X$ são isotrópicos, $Z$ é ortogonal a $\mathbb{R} X \oplus \mathbb{R} T$ e $g(X, T)=g(Z, Z)=1$. O grupo $G$ dessa geometria é $G=\mathrm{Iso}(\mathrm{Sol})$.

Estamos interessados, neste capítulo, em obter um teorema de imersão isométrica nos grupos de Lie $\mathbb{R}^{3}, \mathrm{SL}_{2}(\mathbb{R})$, Nil e Sol. A geometria de Minkowski, por ter curvatura seccional constante, pode ser vista como um caso particular do Exemplo 4.5.4. Ou seja, a $G$-estrutura considerada em $\mathbb{R}^{3}$ é a $G$-estrutura $P$ do Exemplo 4.5.4 que torna $\left(\mathbb{R}^{3}, \nabla, P\right)$ infinitesimalmente homogênea. $\mathrm{O}$ caso de $\mathrm{SL}_{2}(\mathbb{R})$, embora também tenha curvatura seccional 
constante, tem uma $G$-estrutura mais interessante que $\mathbb{R}^{3}$. Assim, $\mathrm{SL}_{2}(\mathbb{R})$ será estudada separadamente do Exemplo 4.5.4.

Os casos mais interessantes são os grupos Nil e Sol, que não possuem curvatura seccional constante. Obteremos um teorema de imersão isométrica para cada um desses grupos.

\section{$5.2 \quad$ O grupo Nil}

\subsubsection{Preliminares}

Consideremos o grupo de Heisenberg Nil munido da métrica Lorentziana invariante à esquerda

$$
\bar{g}=-\mathrm{d} x^{2}+\mathrm{d} y^{2}+(\mathrm{d} z-x \mathrm{~d} y)^{2},
$$

que também será denotada por $\langle\cdot, \cdot\rangle$. Os campos vetoriais

$$
\begin{aligned}
& E_{1}=\frac{\partial}{\partial z} \\
& E_{2}=\frac{\partial}{\partial y}+x \frac{\partial}{\partial z}, \\
& E_{3}=\frac{\partial}{\partial x},
\end{aligned}
$$

constituem uma base para a álgebra de Lie nil de Nil (cf. Exemplo 1.4.8), com

$$
\left\langle E_{1}, E_{1}\right\rangle=\left\langle E_{2}, E_{2}\right\rangle=-\left\langle E_{3}, E_{3}\right\rangle=1 .
$$

Além disso, se $\bar{\nabla}$ denota a conexão de Levi-Civita da métrica $\bar{g}$ dada em (5.2), temos:

$$
\bar{\nabla}=\frac{1}{2}\left(\begin{array}{ccc}
0 & -E_{3} & -E_{2} \\
-E_{3} & 0 & -E_{1} \\
-E_{2} & E_{1} & 0
\end{array}\right)
$$

onde o $(i, j)$-ésimo elemento da matriz acima é $\bar{\nabla}_{E_{i}} E_{j}$ em relação à base ortonormal $\left\{E_{1}, E_{2}, E_{3}\right\}$. De fato, como os colchetes de Lie são dados por

$$
\left[E_{1}, E_{2}\right]=\left[E_{1}, E_{3}\right]=0 \quad \text { e } \quad\left[E_{3}, E_{2}\right]=E_{1},
$$

a matriz (5.5) segue da fórmula de Koszul. 
Observação 5.2.1 A métrica $\bar{g}$, dada em (5.2), é geodesicamente completa. Este fato está demonstrado na Seção 4 de [28], onde as geodésicas são apresentadas explicitamente. Mais geralmente, toda métrica Lorentziana invariante à esquerda em Nil é geodesicamente completa (ver [12]).

Denotando por $\bar{R}$ o tensor de curvatura de Nil, temos a seguinte:

Proposição 5.2.2 Para quaisquer $X, Y, Z \in \boldsymbol{\Gamma}(T$ Nil $)$, temos:

$$
\bar{R}(X, Y) Z=\frac{3}{4} R_{\mathrm{o}}(X, Y) Z+R_{1}(X, Y) Z,
$$

onde

$$
R_{\mathrm{o}}(X, Y) Z=(X \wedge Y) Z
$$

e

$$
R_{1}(X, Y) Z=\left(\left\langle Y, E_{1}\right\rangle X \wedge E_{1}-\left\langle X, E_{1}\right\rangle Y \wedge E_{1}\right) Z
$$

Demonstração. Dado $X \in \boldsymbol{\Gamma}(T \mathrm{Nil})$, escrevemos

$$
X=\widetilde{X}+x E_{1},
$$

onde $\widetilde{X}$ é horizontal ${ }^{2}$ e $x=\left\langle X, E_{1}\right\rangle$. Analogamente escrevemos $Y=\widetilde{Y}+y E_{1}$ e $Z=\widetilde{Z}+z E_{1}$. Mostremos inicialmente que

$$
\bar{R}(\widetilde{X}, \widetilde{Y}) \widetilde{Z}=\frac{3}{4}\left(R_{\mathrm{O}}(X, Y) Z+R_{1}(X, Y) Z\right),
$$

para quaisquer $X, Y, Z \in \boldsymbol{\Gamma}(T \mathrm{Nil})$. De (5.5), temos:

$$
\bar{R}\left(E_{2}, E_{3}\right) E_{2}=\frac{3}{4} E_{3} \quad \text { e } \bar{R}\left(E_{2}, E_{3}\right) E_{3}=\frac{3}{4} E_{2} .
$$

Escrevendo

$$
X=\sum_{i=1}^{3} x_{i} E_{i}, \quad Y=\sum_{j=1}^{3} y_{j} E_{j}, \quad Z=\sum_{k=1}^{3} z_{k} E_{k},
$$

temos:

$$
\bar{R}(\widetilde{X}, \widetilde{Y}) \widetilde{Z}=\frac{3}{4}\left(x_{2} y_{3} z_{2} E_{3}+x_{2} y_{3} z_{3} E_{2}-x_{3} y_{2} z_{2} E_{3}-x_{3} y_{2} z_{3} E_{2}\right) .
$$

\footnotetext{
${ }^{2}$ Aqui estamos considerando Nil como uma fibração sobre o plano de Minkowski; o campo $E_{1}$ é o campo vertical da fibração.
} 
Assim, denotando $\epsilon_{1}=\epsilon_{2}=1$ e $\epsilon_{3}=-1$, temos:

$$
\begin{aligned}
R_{\mathrm{o}}(X, Y) Z= & (X \wedge Y) Z=\sum_{i=1}^{3}\left(\epsilon_{i} x_{i} z_{i} Y-\epsilon_{i} y_{i} z_{i} X\right) \\
= & \sum_{i, j=1}^{3}\left(y_{j} \epsilon_{i} x_{i} z_{i}-x_{j} \epsilon_{i} y_{i} z_{i}\right) E_{j} \\
= & \left(\left\langle Y, E_{1}\right\rangle\langle X, Z\rangle-\left\langle X, E_{1}\right\rangle\langle Y, Z\rangle\right) E_{1}+\left(x_{1} z_{1} y_{2}-y_{1} z_{1} x_{2}\right) E_{2} \\
& +\left(x_{1} z_{1} y_{3}-y_{1} z_{1} x_{3}\right) E_{3}+\left(x_{2} y_{3} z_{3}-x_{3} y_{2} z_{3}\right) E_{2} \\
& +\left(x_{2} y_{3} z_{2}-x_{3} y_{2} z_{2}\right) E_{3} \\
= & \left\langle Y, E_{1}\right\rangle\langle X, Z\rangle E_{1}-\left\langle X, E_{1}\right\rangle\langle Y, Z\rangle E_{1}+\frac{4}{3} \bar{R}(\widetilde{X}, \widetilde{Y}) \widetilde{Z} \\
& +x_{1} z_{1}\left(y_{2} E_{2}+y_{3} E_{3}\right)-y_{1} z_{1}\left(x_{2} E_{2}+x_{3} E_{3}\right) \\
= & \frac{4}{3} \bar{R}(\widetilde{X}, \widetilde{Y}) \widetilde{Z}+\left\langle Y, E_{1}\right\rangle\langle X, Z\rangle E_{1}-\left\langle X, E_{1}\right\rangle\langle Y, Z\rangle E_{1} \\
& +\left\langle X, E_{1}\right\rangle\left\langle Z, E_{1}\right\rangle Y-\left\langle Y, E_{1}\right\rangle\left\langle Z, E_{1}\right\rangle X
\end{aligned}
$$

o que mostra a igualdade (5.8). Provemos agora (5.7). Sejam $X, Y, Z, W \in$ $\boldsymbol{\Gamma}(T \mathrm{Nil})$. Usando a linearidade de $\bar{R}$, o produto $\langle\bar{R}(X, Y) Z, W\rangle$ torna-se uma soma de 16 termos. Os termos onde $E_{1}$ aparece três ou quatro vezes, ou duas vezes nas posições 1,2 ou 3,4 anulam-se pela anti-simetria de $\bar{R}$. Os termos onde $E_{1}$ aparece uma única vez anulam-se pois

$$
\bar{R}\left(E_{1}, E_{i}\right) E_{j}=\bar{R}\left(E_{i}, E_{1}\right) E_{j}=\bar{R}\left(E_{i}, E_{j}\right) E_{1}=0,
$$

para quaisquer $1 \leq i, j \leq 3$. Assim, temos:

$$
\begin{aligned}
\langle\bar{R}(X, Y) Z, W\rangle= & \langle\bar{R}(\widetilde{X}, \widetilde{Y}) \widetilde{Z}, \widetilde{W}\rangle+y w\left\langle\bar{R}\left(\widetilde{X}, E_{1}\right) \widetilde{Z}, E_{1}\right\rangle \\
& +y z\left\langle\bar{R}\left(\widetilde{X}, E_{1}\right) E_{1}, \widetilde{W}\right\rangle+x w\left\langle\bar{R}\left(E_{1}, \widetilde{Y}\right) \widetilde{Z}, E_{1}\right\rangle \\
& +x z\left\langle\bar{R}\left(E_{1}, \widetilde{Y}\right) E_{1}, \widetilde{W}\right\rangle \\
= & \frac{3}{4}\left\langle R_{\mathrm{o}}(X, Y) Z+R_{1}(X, Y) Z, \widetilde{W}\right\rangle \\
& +\frac{1}{4}(-y w\langle\widetilde{X}, \widetilde{Z}\rangle+y z\langle\widetilde{X}, \widetilde{W}\rangle+x w\langle\widetilde{Y}, \widetilde{Z}\rangle-x z\langle\widetilde{Y}, \widetilde{W}\rangle) .
\end{aligned}
$$

Observe agora que:

$$
\left\langle R_{\mathrm{o}}(X, Y) Z+R_{1}(X, Y) Z, E_{1}\right\rangle=0
$$

e

$$
-y w\langle\widetilde{X}, \widetilde{Z}\rangle+y z\langle\widetilde{X}, \widetilde{W}\rangle+x w\langle\widetilde{Y}, \widetilde{Z}\rangle-x z\langle\widetilde{Y}, \widetilde{W}\rangle=\left\langle R_{1}(X, Y) Z, W\right\rangle
$$


Assim,

$$
\begin{aligned}
\langle\bar{R}(X, Y) Z, W\rangle & =\frac{3}{4}\left\langle R_{\mathrm{o}}(X, Y) Z+R_{1}(X, Y) Z, W\right\rangle+\frac{1}{4}\left\langle R_{1}(X, Y) Z, W\right\rangle \\
& =\frac{3}{4}\left\langle R_{\mathrm{o}}(X, Y) Z, W\right\rangle+\left\langle R_{1}(X, Y) Z, W\right\rangle
\end{aligned}
$$

como queríamos.

\subsubsection{As equações de compatibilidade para superfícies em Nil}

Seja $\left\{e_{1}, e_{2}, e_{3}\right\}$ a base canônica do espaço de Minkowski $\mathbb{R}_{1}^{3}$, com $e_{3}$ tipo-tempo. O conjunto

$$
\bar{P}=\mathrm{FR}_{+}^{\mathrm{o}}\left(T \mathrm{Nil} ; e_{1}, E_{1}\right)
$$

é uma $G$-estrutura em Nil, onde $G=\mathrm{SO}\left(\mathbb{R}_{1}^{3} ; e_{1}\right)$ (cf. Exemplo 3.2.5). Mais precisamente, dado $x \in \mathrm{Nil}$, a fibra $\bar{P}_{x}$ é o conjunto das isometrias lineares $p: \mathbb{R}_{1}^{3} \rightarrow T_{x}$ Nil que preservam orientação tais que $p\left(e_{1}\right)=E_{1}(x)$. Por outro lado, sabemos que

$$
\mathrm{O}\left(\mathbb{R}_{1}^{3}\right)=\mathrm{O}(1,2)=\left\{X \in \mathrm{GL}\left(\mathbb{R}^{3}\right): X^{t} \epsilon X=\epsilon\right\},
$$

onde $\epsilon$ é a matriz diagonal $\epsilon=\left(\delta_{i j} \epsilon_{i}\right)$, com $\epsilon_{1}=\epsilon_{2}=1$ e $\epsilon_{3}=-1$. Assim,

$$
G=\left\{X \in \mathrm{GL}\left(\mathbb{R}^{3}\right): X=\left(\begin{array}{cc}
1 & 0 \\
0 & T
\end{array}\right), \quad T \in \mathrm{SO}(1,1)\right\} .
$$

Fazendo $T=\left(\begin{array}{ll}a & b \\ c & d\end{array}\right)$, com $a, b, c, d \in \mathbb{R}$, segue que $T \in \mathrm{SO}(1,1)$ se, e somente se, $a=d, b=c$ e $a^{2}-b^{2}=1$. Portanto, $X \in G$ é da forma

$$
X=\left(\begin{array}{ccc}
1 & 0 & 0 \\
0 & a & b \\
0 & b & a
\end{array}\right) \text {. }
$$

$\mathrm{O}$ referencial $p_{\mathrm{o}} \in \mathrm{FR}^{\mathrm{o}}(T \mathrm{Nil})$, dado por $p_{\mathrm{o}}\left(e_{i}\right)=E_{i}, 1 \leq i \leq 3$, pertence a $G$-estrutura $\bar{P}$. Assim, como a ação é livre e transitiva, segue que, para todo $p \in \bar{P}$, existe um único $g \in G$ tal que $p=p_{\mathrm{o}} \circ g$. Portanto, temos:

$$
\begin{aligned}
& p\left(e_{1}\right)=E_{1}, \\
& p\left(e_{2}\right)=a E_{2}+b E_{3}, \\
& p\left(e_{3}\right)=b E_{2}+a E_{3},
\end{aligned}
$$

com $a, b \in \mathbb{R}$ tais que $a^{2}-b^{2}=1$. Com a notação fixada acima, temos a seguinte 
Proposição 5.2.3 (Nil, $\bar{\nabla}, \bar{P})$ é infinitesimalmente homogênea.

Demonstração. A igualdade (5.7) mostra que o tensor de curvatura $\bar{R}$ de Nil depende somente da métrica, portanto é constante nos referenciais que pertencem a $G$-estrutura $\bar{P}$. Como $\bar{\nabla}$ é compatível com a métrica, a inner torsion é dada por

$$
\mathfrak{I}_{x}^{\bar{P}}(X)=\bar{\nabla}_{X} E_{1},
$$

para quaisquer $x \in$ Nil e $X \in T_{x}$ Nil (cf. Exemplo 3.3.12). Neste caso, a condição da inner torsion é equivalente a existência de uma aplicação linear $L_{\mathrm{o}}: \mathbb{R}^{3} \rightarrow \mathbb{R}^{3}$ tal que

$$
p \circ L_{\mathrm{o}}=\mathfrak{I}_{x}^{\bar{P}} \circ p
$$

para quaisquer $x \in$ Nil e $p \in \bar{P}_{x}$ (cf. Exemplo 4.5.8); basta definir $L_{\mathrm{O}}: \mathbb{R}^{3} \rightarrow$ $\mathbb{R}^{3}$ por:

$$
\begin{aligned}
& L_{\mathrm{o}}\left(e_{1}\right)=0 \\
& L_{\mathrm{o}}\left(e_{2}\right)=-\frac{1}{2} e_{3}, \\
& L_{\mathrm{o}}\left(e_{3}\right)=-\frac{1}{2} e_{2} .
\end{aligned}
$$

A relação (5.12) segue agora de (5.10) e (5.11). Assim, a condição da inner torsion está satisfeita. A condição da torção é trivialmente satisfeita pois $\bar{T}=0$.

Consideremos agora uma variedade Riemanniana $(M, g)$ de dimensão 2 , $E=M \times \mathbb{R}$ o fibrado vetorial trivial sobre $M$ munido de uma métrica semiRiemanniana $g^{E}$ de índice $1, \nabla^{E}$ uma conexão em $E$ compatível com $g^{E}$ e $\alpha$ uma seção diferenciável simétrica de $\operatorname{Lin}_{2}(T M ; E)$. No fibrado vetorial $\widehat{E}=T M \oplus E$, consideremos a métrica semi-Riemanniana $\widehat{g}$ dada pela soma direta ortogonal de $g$ e $g^{E}$, e $\widehat{\nabla}$ a conexão em $\widehat{E}$ cujas componentes são $\nabla$, $\nabla^{E}$ e $\alpha$. Além disso, consideremos a $G$-estrutura

$$
\widehat{P}=\mathrm{FR}_{+}^{\mathrm{o}}\left(\widehat{E} ; e_{1},(T, \nu)\right)
$$

onde $T \in \boldsymbol{\Gamma}(T M)$ e $\nu \in \mathrm{C}^{\infty}(M)$ são tais $\|T\|^{2}+\nu^{2}=1$.

Suponha a existência de uma imersão isométrica $(f, S)$ de $M$ em Nil que preserva $G$-estrutura. 
Proposição 5.2.4 Para quaisquer $X, Y, Z, W \in \boldsymbol{\Gamma}(T \mathrm{Nil})$, temos:

$$
\langle\bar{R}(X, Y) Z, W\rangle=\frac{3}{4}\left\langle R_{\mathrm{o}}(X, Y) Z, W\right\rangle+\left\langle R_{1}(X, Y) Z, W\right\rangle
$$

e

$$
\bar{R}(X, Y) N=\nu(Y \wedge X) T
$$

onde $N$ é o campo normal unitário a $M$ e $R_{0}, R_{1}$ são como na Proposição 5.2 .2 .

Demonstração. Segue da Proposição 5.2.2, usando o fato que $X, Y, Z$ são tangentes à variedade $M, N$ é normal à variedade $M$ e $\nu$ é a componente normal de $T$, i.e., $\nu=\langle T, N\rangle$.

Corolário 5.2.5 As equações de Gauss e Codazzi em Nil são

$$
K=\nu^{2}-\operatorname{det}(A)-\frac{1}{4}
$$

e

$$
\nabla_{X} A Y-\nabla_{Y} A X-A([X, Y])=\nu(Y \wedge X) T
$$

respectivamente, onde $K$ é a curvatura Gaussiana de $M$ e $A: \boldsymbol{\Gamma}(T M) \rightarrow$ $\boldsymbol{\Gamma}(T M)$ é o operador de Weingarten associado a $N$.

Demonstração. Como a codimensão é 1, as equações de Gauss (equação (4.25)) e Codazzi (equação (4.26)) tornam-se

$$
\langle\bar{R}(X, Y) Z, W\rangle=\langle R(X, Y) Z, W\rangle+\langle(A X \wedge A Y) Z, W\rangle
$$

e

$$
\bar{R}(X, Y) N=\nabla_{X} A Y-\nabla_{Y} A X-A([X, Y]),
$$

respectivamente (cf. Exemplo 4.3.5). Dado $x \in M$, fixemos uma base ortonormal $\{X, Y\}$ de $T_{x} M$. De (5.14), temos $\langle\bar{R}(X, Y) X, Y\rangle=\nu^{2}-\frac{1}{4}$. Além disso, temos:

$$
\langle R(X, Y) X, Y\rangle=K \quad \text { e } \quad\langle(A X \wedge A Y) X, Y\rangle=\operatorname{det}(A),
$$

obtendo, assim, a equação (5.16). A equação de Codazzi (5.17) segue diretamente de (5.15). 


\subsubsection{Imersões isométricas em Nil}

Consideremos uma variedade Riemanniana $(M, g)$ de dimensão $2, \nabla$ sua conexão de Levi-Civita, $R$ o tensor de curvatura e $K$ sua curvatura Gaussiana. Seja $E=T M \times \mathbb{R}$ o fibrado vetorial trivial sobre $M$ munido de uma métrica semi-Riemanniana $g^{E}$ e denotemos por $\nabla^{E}$ uma conexão em $E$ compatível com $g^{E}$. Dado uma seção diferenciável simétrica $\alpha$ do fibrado vetorial $\operatorname{Lin}_{2}(T M ; E)$, denotemos por $\widehat{g}$ a métrica semi-Riemanniana no fibrado $\widehat{E}=T M \oplus E$ dada pela soma direta ortogonal de $g$ e $g^{E}$, e consideremos a conexão $\widehat{\nabla}$ em $\widehat{E}$ cujas componentes são $\nabla, \nabla^{E}$ e $\alpha$.

Sejam $T \in \boldsymbol{\Gamma}(T M)$ e $\nu \in C^{\infty}(M)$ tais que $\|T\|^{2}+\nu^{2}=1$. As equações de compatibilidade para superfícies no grupo de Heisenberg Lorentziano estabelecidas na Seção 5.2.2 sugerem a seguinte

Definição 5.2.6 Dizemos que a terna $(M, \alpha,(T, \nu))$ satisfaz as equações de compatibilidade para $(\mathrm{Nil}, \bar{g})$ se as seguintes condições são satisfeitas:

(a) Para todo $x \in M$, existe uma isometria linear $p_{\mathrm{o}}: \mathbb{R}_{1}^{3} \rightarrow \widehat{E}_{x}$, com $p_{\mathrm{o}}\left(e_{1}\right)=(T(x), \nu(x))$, tal que:

$$
\left.p_{\mathrm{o}} \circ L_{\mathrm{o}}\right|_{p_{\mathrm{o}}^{-1}\left(T_{x} M\right)}=\left.(\widehat{\nabla}(T, \nu))_{x} \circ p_{\mathrm{o}}\right|_{p_{\mathrm{o}}^{-1}\left(T_{x} M\right)},
$$

onde $L_{\mathrm{o}}: \mathbb{R}_{1}^{3} \rightarrow \mathbb{R}_{1}^{3}$ é a aplicação linear definida por

$$
\begin{aligned}
& L_{\mathrm{o}}\left(e_{1}\right)=0, \\
& L_{\mathrm{o}}\left(e_{2}\right)=-\frac{1}{2} e_{3}, \\
& L_{\mathrm{o}}\left(e_{3}\right)=-\frac{1}{2} e_{2} .
\end{aligned}
$$

(b) Vale a equação de Gauss:

$$
K=\nu^{2}-\operatorname{det}(A)-\frac{1}{4},
$$

onde $A_{x}: T_{x} M \rightarrow T_{x} M$ é definido por $g(A X, Y)=g^{E}(\alpha(X, Y), \eta)$, para alguma seção unitária $\eta$ de $E$.

(c) Vale a equação de Codazzi:

$$
\nabla_{X} A Y-\nabla_{Y} A X-A([X, Y])=\nu(X \wedge Y) T .
$$

Seguindo a notação acima, temos o seguinte 
Teorema 5.2.7 Suponha que a terna $(M, \alpha,(T, \nu))$ satisfaça as equações de compatibilidade da Definição 5.2.6. Então, para quaisquer $x_{\mathrm{o}} \in M e$ $y_{\mathrm{o}} \in \mathrm{Nil}$, existem uma vizinhança $U$ de $x_{\mathrm{o}}$ em $M$ e uma imersão isométrica $f: U \rightarrow$ Nil tal que o operador de Weingarten A de $f$ em relação ao normal $N$ é dado por

$$
\mathrm{d} f \circ A \circ \mathrm{d} f^{-1}
$$

e tal que

$$
E_{1}=\mathrm{d} f(T)+\nu N
$$

Além disso, se $M$ é conexa e simplesmente conexa, existe uma imersão isométrica global $f: M \rightarrow$ Nil satisfazendo (5.21) e (5.22).

Demonstração. Denotando por $\epsilon=(T, \nu) \in \boldsymbol{\Gamma}(\widehat{E})$, consideremos a $G$ estrutura

$$
\widehat{P}=\mathrm{FR}_{+}^{\mathrm{o}}\left(\widehat{E} ; e_{1}, \epsilon\right)
$$

em $\widehat{E}$, onde $G=\mathrm{SO}\left(\mathbb{R}_{1}^{3} ; e_{1}\right)$. A hipótese (4.28) do Teorema 4.5.2 significa, neste caso, que

$$
\left.p \circ L_{\mathrm{o}}\right|_{p^{-1}\left(T_{x} M\right)}=\left.(\widehat{\nabla} \epsilon)_{x} \circ p\right|_{p^{-1}\left(T_{x} M\right)},
$$

para quaisquer $x \in M$ e $p \in \widehat{P}_{x}$. Como $(M, \alpha,(T, \nu))$ satisfaz as equações de compatibilidade, existe $p_{\mathrm{o}}: \mathbb{R}_{1}^{3} \rightarrow T_{x} \mathrm{Nil}$, com $p_{\mathrm{o}}\left(e_{1}\right)=\epsilon(x)$, tal que vale (5.23). Segue, em particular, que $p_{\mathrm{o}} \in \widehat{P}$. Por outro lado, todo referencial $p \in \widehat{P}$ se escreve como $p=p_{\text {o }} \circ g$, para algum $g \in G$. Representando matricialmente

$$
g=\left(\begin{array}{ccc}
1 & 0 & 0 \\
0 & a & b \\
0 & b & a
\end{array}\right), \quad L_{\mathrm{o}}=\left(\begin{array}{ccc}
0 & 0 & 0 \\
0 & 0 & -\frac{1}{2} \\
0 & -\frac{1}{2} & 0
\end{array}\right),
$$

com $a^{2}-b^{2}=1$, um cálculo simples mostra que $g \circ L_{\mathrm{o}} \circ g^{-1}=L_{\mathrm{o}}$. Assim,

$$
\begin{aligned}
\left.p \circ L_{\mathrm{o}} \circ p^{-1}\right|_{T_{x} M} & =\left.p_{\mathrm{o}} \circ g \circ L_{\mathrm{o}} \circ g^{-1} \circ p_{\mathrm{o}}^{-1}\right|_{T_{x} M}=\left.p_{\mathrm{o}} \circ L_{\mathrm{o}} \circ p_{\mathrm{o}}^{-1}\right|_{T_{x} M} \\
& =(\nabla \epsilon)_{x},
\end{aligned}
$$

mostrando que a condição (5.23) vale, de fato, para todo $p \in \widehat{P}_{x}$. Finalmente, as equações de compatibilidade (5.19) e (5.20) claramente implicam as equações (4.25) e (4.26), respectivamente. A versão global segue diretamente do Teorema 4.5.2, pois a métrica $\bar{g}$ de Nil é geodesicamente completa (cf. Observação 5.2.1). 


\subsection{O grupo $\mathrm{SL}_{2}(\mathbb{R})$}

\subsubsection{Preliminares}

Consideremos o grupo de Lie $\mathrm{SL}_{2}(\mathbb{R})$, cuja álgebra de Lie $\mathfrak{s l}_{2}(\mathbb{R})$ consiste das matrizes reais $2 \times 2$ de traço zero (cf. Exemplo 1.4.5). A forma de Killing, definida em (1.14), na álgebra de Lie $\mathfrak{s l}_{2}(\mathbb{R})$ é dada por

$$
\bar{g}(X, Y)=\frac{1}{2} \operatorname{tr}(X Y)
$$

para quaisquer $X, Y \in \mathfrak{s l}_{2}(\mathbb{R})$ (ver [13, Seção 4]). As matrizes

$$
\left(\begin{array}{cc}
1 & 0 \\
0 & -1
\end{array}\right),\left(\begin{array}{cc}
0 & 1 \\
1 & 0
\end{array}\right),\left(\begin{array}{cc}
0 & -1 \\
1 & 0
\end{array}\right)
$$

são ortogonais e têm comprimentos iguais a 1,1 e -1 , respectivamente. Assim, (5.24) é um produto escalar Lorentziano em $\mathfrak{s l}_{2}(\mathbb{R})$. Dado $X \in$ $\mathfrak{s l}_{2}(\mathbb{R}), \mathrm{com}$

$$
X=\left(\begin{array}{cc}
x_{1} & x_{2} \\
x_{3} & -x_{1}
\end{array}\right)
$$

$x_{1}, x_{2}, x_{3} \in \mathbb{R}$, temos

$$
g(X, X)=x_{1}^{2}+x_{2} x_{3} .
$$

Identificando a matriz $X$ com o vetor $\left(x_{1}, x_{2}, x_{3}\right) \in \mathbb{R}^{3}$, a métrica (5.24) pode ser escrita como

$$
\bar{g}=d x_{1}^{2}+d x_{2} d x_{3} .
$$

Além disso, as matrizes em (5.25) podem ser identificadas com os campos vetoriais

$$
\begin{aligned}
& X_{1}=\frac{\partial}{\partial x} \\
& X_{2}=\frac{\partial}{\partial y}+\frac{\partial}{\partial z} \\
& X_{3}=-\frac{\partial}{\partial y}+\frac{\partial}{\partial z}
\end{aligned}
$$

que constituem uma base ortonormal para $\mathfrak{s l}_{2}(\mathbb{R})$, com

$$
\bar{g}\left(X_{1}, X_{1}\right)=\bar{g}\left(X_{2}, X_{2}\right)=-\bar{g}\left(X_{3}, X_{3}\right)=1 .
$$

Se $\bar{\nabla}$ denota a conexão de Levi-Civita da métrica (5.24), temos:

$$
\bar{\nabla}=\left(\begin{array}{ccc}
0 & X_{3} & X_{2} \\
-X_{3} & 0 & -X_{1} \\
-X_{2} & X_{1} & 0
\end{array}\right),
$$


onde o $(i, j)$-ésimo elemento da matriz (5.26) é $\bar{\nabla}_{E_{i}} E_{j}$ em relação à base ortonormal $\left\{X_{1}, X_{2}, X_{3}\right\}$. De fato, um cálculo simples mostra que os colchetes de Lie dos campos $X_{1}, X_{2}, X_{3}$ são dados por

$$
\left[X_{1}, X_{2}\right]=2 X_{3}, \quad\left[X_{1}, X_{3}\right]=2 X_{2} \quad \text { e } \quad\left[X_{2}, X_{3}\right]=-2 X_{1} .
$$

A matriz (5.26) segue agora da fórmula de Koszul.

Observação 5.3.1 Seja $G$ um grupo de Lie com álgebra de Lie $\mathfrak{g}$. A condição (??), que a forma de Killing $\langle\cdot, \cdot\rangle$ de $\mathfrak{g}$ satisfaz, é equivalente a $\langle\cdot, \cdot\rangle$ ser $\operatorname{Ad}(G)$-invariante. Esse fato, por sua vez, é equivalente às geodésicas de $G$ partindo de $1 \in G$ serem os subgrupos a 1-parâmetro de $G$ (ver [23, Proposição 11.9]). Segue, em particular, que o grupo $G$, munido da forma de Killing, é geodesicamente completo.

Proposição 5.3.2 O tensor de curvatura $\bar{R}$ de $\mathrm{SL}_{2}(\mathbb{R})$ é dado por

$$
\bar{R}(X, Y) Z=-(X \wedge Y) Z,
$$

para quaisquer $X, Y, Z \in \mathrm{SL}_{2}(\mathbb{R})$, ou seja, $\mathrm{SL}_{2}(\mathbb{R})$ tem curvatura seccional constante igual a -1 .

Demonstração. Um cálculo simples, usando as derivadas covariantes (5.26), mostra que a igualdade (5.27) vale na álgebra de Lie $\mathfrak{s l}_{2}(\mathbb{R})$. Como a métrica em $\mathrm{SL}_{2}(\mathbb{R})$ é obtida a partir da métrica da álgebra de Lie $\mathfrak{s l}_{2}(\mathbb{R})$ por translações à esquerda, obtemos então a expressão (5.27). Note que, como a forma de Killing (5.24) é bi-invariante, já sabíamos que o tensor de curvatura $\bar{R}$ é dado por

$$
\bar{R}(X, Y) Z=[[X, Y], Z],
$$

para quaisquer $X, Y, Z \in \mathfrak{s l}_{2}(\mathbb{R})$ (ver [23, Corolário 11.10]).

Observação 5.3.3 Como $\mathrm{SL}_{2}(\mathbb{R})$ tem curvatura seccional constante, obtemos de imediato, pelo Exemplo 4.5.4, um teorema de imersão isométrica em $\mathrm{SL}_{2}(\mathbb{R})$. No entanto, usando a estrutura da álgebra de Lie $\mathfrak{s l}_{2}(\mathbb{R})$, podemos definir uma $G$-estrutura $\bar{P}$ em $\mathrm{SL}_{2}(\mathbb{R})$ tal que $\left(\mathrm{SL}_{2}(\mathbb{R}), \bar{\nabla}, \bar{P}\right)$ seja infinitesimalmente homogênea, porém com a inner torsion não-nula. Tal $G$-estrutura é semelhante à $G$-estrutura considerada no grupo de Heisenberg. 


\subsubsection{As equações de compatibilidade para superfícies em $\mathrm{SL}_{2}(\mathbb{R})$}

Seja $\left\{e_{1}, e_{2}, e_{3}\right\}$ a base canônica do espaço de Minkowski $\mathbb{R}_{1}^{3}$, com $e_{3}$ tipo-tempo. O conjunto

$$
\bar{P}=\mathrm{FR}_{+}^{\mathrm{o}}\left(T \mathrm{SL}_{2}(\mathbb{R}) ; e_{1}, X_{1}\right)
$$

é uma $G$-estrutura em $\mathrm{SL}_{2}(\mathbb{R})$, onde $G=\mathrm{SO}\left(\mathbb{R}_{1}^{3} ; e_{1}\right)$ (cf. Exemplo 3.2.5). Como visto na Subseção 5.2.2, para todo $p \in \bar{P}$, existe um único $g \in G$ tal que $p=p_{0} \circ g$, onde $p_{0} \in \bar{P}$ é dado por $p_{0}\left(e_{i}\right)=X_{i}, 1 \leq i \leq 3$. Assim,

$$
\begin{aligned}
& p\left(e_{1}\right)=X_{1}, \\
& p\left(e_{2}\right)=a X_{2}+b X_{3}, \\
& p\left(e_{3}\right)=b X_{2}+a X_{3},
\end{aligned}
$$

com $a, b \in \mathbb{R}$ tais que $a^{2}-b^{2}=1$.

Proposição 5.3.4 $\left(\mathrm{SL}_{2}(\mathbb{R}), \bar{\nabla}, \bar{P}\right)$ é infinitesimalmente homogênea.

Demonstração. A igualdade (5.27) mostra que $\bar{R}$ é constante nos referenciais que pertencem a $G$-estrutura (5.28). A condição da inner torsion segue de forma inteiramente análoga ao caso do grupo de Heisenberg. De fato, neste caso temos:

$$
\mathfrak{I}_{x}^{\bar{P}}(X)=\bar{\nabla}_{X} X_{1},
$$

para quaisquer $x \in \mathrm{SL}_{2}(\mathbb{R})$ e $X \in T_{x} \mathrm{SL}_{2}(\mathbb{R})$. Finalmente, a condição da torção é trivialmente satisfeita pois $\bar{T}=0$.

Consideremos agora uma variedade Riemanniana $(M, g)$ de dimensão 2, $E=M \times \mathbb{R}$ o fibrado vetorial trivial sobre $M$ munido de uma métrica semiRiemanniana $g^{E}$ de índice $1, \nabla^{E}$ uma conexão em $E$ compatível com $g^{E}$ e $\alpha$ uma seção diferenciável simétrica de $\operatorname{Lin}_{2}(T M ; E)$. No fibrado vetorial $\widehat{E}=T M \oplus E$, consideremos a métrica semi-Riemanniana $\widehat{g}$ dada pela soma direta ortogonal de $g$ e $g^{E}$, e $\widehat{\nabla}$ a conexão em $\widehat{E}$ cujas componentes são $\nabla$, $\nabla^{E}$ e $\alpha$. Além disso, consideremos a $G$-estrutura

$$
\widehat{P}=\mathrm{FR}_{+}^{\mathrm{o}}\left(\widehat{E} ; e_{1},(T, \nu)\right)
$$

onde $T \in \boldsymbol{\Gamma}(T M)$ e $\nu \in \mathrm{C}^{\infty}(M)$ são tais $\|T\|^{2}+\nu^{2}=1$.

Suponha a existência de uma imersão isométrica $(f, S)$ de $M$ em $\mathrm{SL}_{2}(\mathbb{R})$ que preserva $G$-estrutura. 
Proposição 5.3.5 As equações de Gauss e Codazzi em $\mathrm{SL}_{2}(\mathbb{R})$ são dadas por

$$
K=-\operatorname{det}(A)-1
$$

e

$$
\nabla_{X} A Y-\nabla_{Y} A X=A([X, Y])
$$

respectivamente, para quaisquer $x \in M$ e $X, Y \in T_{x} M$, onde $K$ denota a curvatura Gaussiana de $M$ e $A: T_{x} M \rightarrow T_{x} M$ denota o operador de Weingarten associado ao campo normal $N$.

Demonstração. Segue diretamente de (5.27).

\subsubsection{Imersões isométricas em $\mathrm{SL}_{2}(\mathbb{R})$}

Consideremos uma variedade Riemanniana $(M, g)$ de dimensão $2, \nabla$ sua conexão de Levi-Civita, $R$ o tensor de curvatura e $K$ sua curvatura Gaussiana. Seja $E=T M \times \mathbb{R}$ o fibrado vetorial trivial sobre $M$ munido de uma métrica semi-Riemanniana $g^{E}$ e denotemos por $\nabla^{E}$ uma conexão em $E$ compatível com $g^{E}$. Dado uma seção diferenciável simétrica $\alpha$ do fibrado vetorial $\operatorname{Lin}_{2}(T M ; E)$, denotemos por $\widehat{g}$ a métrica semi-Riemanniana no fibrado $\widehat{E}=T M \oplus E$ dada pela soma direta ortogonal de $g$ e $g^{E}$, e consideremos a conexão $\widehat{\nabla}$ em $\widehat{E}$ cujas componentes são $\nabla, \nabla^{E}$ e $\alpha$.

Sejam $T \in \boldsymbol{\Gamma}(T M)$ e $\nu \in C^{\infty}(M)$ tais que $\|T\|^{2}+\nu^{2}=1$. Como no caso Lorentziano, consideremos a seguinte

Definição 5.3.6 Dizemos que a terna $(M, \alpha,(T, \nu))$ satisfaz as equações de compatibilidade para $\left(\mathrm{SL}_{2}(R), \bar{g}\right)$ se as seguintes condições são satisfeitas:

(a) Para todo $x \in M$, existe uma isometria linear $p_{\mathrm{o}}: \mathbb{R}_{1}^{3} \rightarrow \widehat{E}_{x}$, com $p_{\mathrm{o}}\left(e_{1}\right)=(T(x), \nu(x))$, tal que:

$$
\left.p_{\mathrm{o}} \circ L_{\mathrm{o}}\right|_{p_{\mathrm{o}}^{-1}\left(T_{x} M\right)}=\left.(\widehat{\nabla}(T, \nu))_{x} \circ p_{\mathrm{o}}\right|_{p_{\mathrm{o}}^{-1}\left(T_{x} M\right)},
$$

onde $L_{\mathrm{o}}: \mathbb{R}_{1}^{3} \rightarrow \mathbb{R}_{1}^{3}$ é a aplicação linear definida por

$$
\begin{aligned}
& L_{\mathrm{o}}\left(e_{1}\right)=0, \\
& L_{\mathrm{o}}\left(e_{2}\right)=-\frac{1}{2} e_{3}, \\
& L_{\mathrm{o}}\left(e_{3}\right)=-\frac{1}{2} e_{2} .
\end{aligned}
$$

(b) Valem as equações de Gauss e Codazzi dadas em (5.29) e (5.30), respectivamente. 
Seguindo a notação acima, temos o seguinte

Teorema 5.3.7 Suponha que a terna $(M, \alpha,(T, \nu))$ satisfaça as equações de compatibilidade da Definição 5.3.6. Então, para quaisquer $x_{\mathrm{o}} \in M$ e $y_{\mathrm{o}} \in$ $\mathrm{SL}_{2}(\mathbb{R})$, existem uma vizinhança $U$ de $x_{\mathrm{o}}$ em $M$ e uma imersão isométrica $f: U \rightarrow \mathrm{SL}_{2}(\mathbb{R})$ tal que o operador de Weingarten $A$ de $f$ em relação ao normal $N$ é dado por

$$
\mathrm{d} f \circ A \circ \mathrm{d} f^{-1}
$$

e tal que

$$
E_{1}=\mathrm{d} f(T)+\nu N
$$

Além disso, se $M$ é conexa e simplesmente conexa, existe uma imersão isométrica global $f: M \rightarrow \mathrm{SL}_{2}(\mathbb{R})$ satisfazendo (5.32) e (5.33).

Demonstração. A prova é inteiramente análoga à demonstração do Teorema 5.2.7.

\subsection{O grupo Sol}

\subsubsection{Preliminares}

Consideremos o grupo de Lie Sol, cuja álgebra de Lie $\mathfrak{s o l}$ é gerada pelos campos invariantes à esquerda $T, X, Z$ que satisfazem

$$
[T, X]=X, \quad[T, Z]=-Z \quad \text { e } \quad[X, Z]=0
$$

(cf. Exemplo 1.4.9). Denotemos por $\bar{g}$ a métrica Lorentziana invariante à esquerda em Sol considerada na classificação das 3-variedades Lorentzianas. Mais precisamente, $\bar{g}$ é definida na álgebra de Lie $\mathfrak{s o l}$ exigindo-se que $T$ e $X$ são isotrópicos, $Z$ é ortogonal ao plano gerado por $T$ e $X$, e $\bar{g}(T, X)=$ $\bar{g}(Z, Z)=1$. Isso de fato define $\bar{g}$ e, matricialmente, $\bar{g}$ é dada por

$$
\bar{g}=\left(\begin{array}{lll}
0 & 1 & 0 \\
1 & 0 & 0 \\
0 & 0 & 1
\end{array}\right) .
$$

Por outro lado, a álgebra de Lie nil do grupo de Heisenberg Nil é gerada pelos campos invariantes à esquerda $X, Z, Y$, cujos colchetes de Lie são dados por

$$
[Y, Z]=X \quad \text { e } \quad[X, Y]=[X, Z]=0
$$


(cf. Exemplo 1.4.8). Denotemos por a a álgebra de Lie de dimensão 1 gerada pelo campo $T$. Consideremos uma representação

$$
\sigma: \mathfrak{a} \rightarrow \mathfrak{g l}(\mathfrak{n i l})
$$

de $\mathfrak{a}$ em $\mathfrak{n i l}$, onde $\sigma(T)$ é definido sobre a base $\{X, Z, Y\}$ de $\mathfrak{n i l}$ por:

$$
\sigma(T)=\operatorname{ad}_{T}=\left(\begin{array}{ccc}
1 & 0 & 0 \\
0 & -1 & 1 \\
0 & 0 & 2
\end{array}\right),
$$

e estendendo por linearidade. De (5.36), temos

$$
[T, Y]=Z+2 Y \text {. }
$$

Mostremos que $\sigma(W)$ é uma derivação de nil para todo $W \in \mathfrak{a}$. Para isso, basta mostrar que $\sigma(T)$ é uma derivação de nil. Dados $W_{1}, W_{2} \in \mathfrak{n i l}$, temos:

$$
W_{1}=\alpha_{1} X+\alpha_{2} Z+\alpha_{3} Y \text { e } W_{2}=\beta_{1} X+\beta_{2} Z+\beta_{3} Y .
$$

Tomando o produto com $T$, obtemos:

$$
\begin{aligned}
{\left[\left[T, W_{1}\right], W_{2}\right] } & =\left[\left[T, \alpha_{1} X+\alpha_{2} Z+\alpha_{3} Y\right], W_{2}\right] \\
& =\left[\alpha_{1} X-\alpha_{2} Z+\alpha_{3}(Z+2 Y), \beta_{1} X+\beta_{2} Z+\beta_{3} Y\right] \\
& =\left(\alpha_{2} \beta_{3}-\alpha_{3} \beta_{3}+2 \alpha_{3} \beta_{2}\right) X \\
{\left[W_{1},\left[T, W_{2}\right]\right] } & =\left[W_{1},\left[T, \beta_{1} X+\beta_{2} Z+\beta_{3} Y\right]\right] \\
& =\left[\alpha_{1} X+\alpha_{2} Z+\alpha_{3} Y, \beta_{1} X-\beta_{2} Z+\beta_{3}(Z+2 Y)\right] \\
& =\left(-2 \alpha_{2} \beta_{3}-\alpha_{3} \beta_{2}+\alpha_{3} \beta_{3}\right) X
\end{aligned}
$$

e

$$
\begin{aligned}
{\left[\left[T, W_{1}\right], W_{2}\right]+\left[W_{1},\left[T, W_{2}\right]\right] } & =\left(\alpha_{3} \beta_{2}-\alpha_{2} \beta_{3}\right) X \\
& =\sigma(T)\left(\left[W_{1}, W_{2}\right]\right)
\end{aligned}
$$

como queríamos. Podemos, assim, formar o produto semi-direto

$$
\mathfrak{n i l} \rtimes \mathfrak{a}
$$

relativo à representação (5.35) (cf. Exemplo 1.2.7). Pelo Lema 1.3.3, existe um único homomorfismo de grupos de Lie

$$
\rho: \mathbb{R} \rightarrow \operatorname{Aut}(\mathrm{Nil})
$$


tal que o produto semi-direto ${ }^{3}$

$$
K=\mathrm{Nil} \rtimes \mathbb{R},
$$

relativo ao homomorfismo (5.38), é o produto semi-direto associado com a álgebra de Lie (5.37). Assim, pelo Lema 1.3.2, nil $\rtimes \mathfrak{a}$ é isomorfa à álgebra de Lie do produto semi-direto (5.39).

Consideremos agora o subgrupo a 1-parâmetro $H=\{\exp (t Y)\}$ de Nil. Como Nil é um grupo de Lie nilpotente simplesmente conexo, $H$ é fechado em Nil (cf. Teorema 1.3.8) e, portanto, $H$ é fechado em $K$. Assim, podemos considerar o espaço homogêneo $K / H$. Por outro lado, o grupo Sol é o produto semi-direto

$$
\text { Sol }=A \rtimes \mathbb{R},
$$

onde $A$ é o grupo de Lie cuja álgebra de Lie é gerada pelos campos $X, Z$. Como $A$ é um subgrupo de Nil, segue que Sol é um subgrupo de $K$. Portanto, a ação canônica de $K$ sobre $K / H$ se restringe a ação

$$
\mathrm{Sol} \times K / H \rightarrow K / H .
$$

Teorema 5.4.1 A ação (5.40) é por isometrias. Mais precisamente, a métrica Lorentziana Sol-invariante considerada em $K / H$ é a métrica $\bar{g}$ dada em (5.34).

Demonstração. O primeiro passo é provar que a ação (5.40) é livre e transitiva. Mostremos inicialmente que Sol $H=K$. Sejam então $(g, t) \in$ $A \times \mathbb{R}$ e $h \in H$. Temos:

$$
(g, t) \cdot(h, 0)=\left(g \cdot \rho_{t}(h), t\right) \in \mathrm{Nil} \times \mathbb{R},
$$

onde $\rho$ é o homomorfismo dado em (5.38). Determinemos o valor $\rho_{t}(h)$, com $t \in \mathbb{R}$ e $h \in H$. Consideremos o homomorfismo de grupos de Lie $\tau: \mathbb{R} \rightarrow \operatorname{Aut}(\mathfrak{n i l})$, definido por

$$
\tau(t)=\tau_{t}=\mathrm{d} \rho_{t}(1) \in \operatorname{Aut}(\mathfrak{n i r} \mathfrak{l}),
$$

para todo $t \in \mathbb{R}$ (cf. Subseção 1.3.1). Denotemos por $\sigma$ a diferencial de $\tau$ no elemento identidade $1 \in \mathbb{R}$. Temos:

$$
\sigma(t)=\mathrm{d} \tau(1)(t)=\frac{d}{d t} \tau_{t},
$$

\footnotetext{
${ }^{3}$ Aqui, estamos identificando, canonicamente, a álgebra de Lie de $\mathbb{R}$ com a álgebra de Lie $\mathfrak{a}$ gerada pelo campo $T$.
} 
para todo $t \in \mathbb{R}$. Assim,

$$
\tau_{t}=\frac{t^{2}}{2} \operatorname{ad}_{T}
$$

Dado $h \in H$, tem-se $h=r Y$, para algum $r \in \mathbb{R}$. Então, usando (1.23), temos:

$$
\begin{aligned}
\rho_{t}(h) & =\rho_{t}(\exp (r Y))=\exp \left(\tau_{t}(r Y)\right)=\exp \left(\frac{t^{2} r}{2} \operatorname{ad}_{T}(Y)\right) \\
& =\exp (c(Z+2 Y))=\exp (c Z) \cdot \exp (2 c Y) \in A \cdot H
\end{aligned}
$$

onde $c=\frac{t^{2} r}{2}$. Mostremos agora que o produto $g \cdot \rho_{t}(h)$ é, de fato, Nil. Consideremos as representações matriciais de $A$ e $H$ dadas por

$$
A=\left(\begin{array}{lll}
1 & 0 & z \\
0 & 1 & y \\
0 & 0 & 1
\end{array}\right) \quad \text { e } H=\left(\begin{array}{lll}
1 & x & 0 \\
0 & 1 & 0 \\
0 & 0 & 1
\end{array}\right)
$$

com $x, y, z \in \mathbb{R}$. Então, usando (5.42), temos:

$$
\begin{aligned}
g \cdot \rho_{t}(h) & =\left(\begin{array}{lll}
1 & 0 & z \\
0 & 1 & y \\
0 & 0 & 1
\end{array}\right)\left(\begin{array}{ccc}
1 & 0 & 0 \\
0 & 1 & \frac{t^{2}}{2} x \\
0 & 0 & 1
\end{array}\right)\left(\begin{array}{lll}
1 & x & 0 \\
0 & 1 & 0 \\
0 & 0 & 1
\end{array}\right) \\
& =\left(\begin{array}{ccc}
1 & 0 & z \\
0 & 1 & \frac{t^{2}}{2} x+y \\
0 & 0 & 1
\end{array}\right)\left(\begin{array}{ccc}
1 & x & 0 \\
0 & 1 & 0 \\
0 & 0 & 1
\end{array}\right) \\
& =\left(\begin{array}{ccc}
1 & x & z \\
0 & 1 & \frac{t^{2}}{2} x+y \\
0 & 0 & 1
\end{array}\right) .
\end{aligned}
$$

A última igualdade acima mostra que o produto $g \cdot \rho_{t}(h)$ gera o grupo todo Nil. Mais precisamente, dado $(a, b, c) \in \mathrm{Nil}$, basta tomar $g=a X+c Z \in A \mathrm{e}$ $h=a\left(1-\frac{t^{2}}{2}\right) Y \in H$. Portanto, mostramos que Sol $H=K$. Para provar que a ação (5.40) é transitiva, sejam $g_{1} H, g_{2} H \in K / H$. Temos que $g_{1}=s_{1} h_{1} \mathrm{e}$ $g_{2}=s_{2} h_{2}$, com $s_{i} \in$ Sol e $h_{i} \in H, i=1,2$. Tomando $s=s_{2} s_{1}^{-1} \in$ Sol, segue que $s \cdot g_{1} H=g_{2} H$. Para mostrar que a ação (5.40) é livre, consideremos um ponto arbitrário $g H \in K / H$. O subgrupo de isotropia de Sol em $g H$ é dado por

$$
\operatorname{Sol}_{g H}=\operatorname{Sol} \cap H \text {. }
$$

Como $H$ é fechado em $K$, a interseção Sol $\cap H$ é fechada em Sol e, assim, Sol $\cap H$ é fechado em $K$. Portanto, Sol $\cap H$ é um subgrupo de Lie de $K$. 
Sua álgebra de Lie é dada por

$$
\{X: \exp (t X) \in \operatorname{Sol} \cap H, t \in \mathbb{R}\}=\mathfrak{s o l} \cap \mathfrak{h}=\{1\},
$$

o que mostra que Sol $\cap H$ é discreto. Por outro lado, como $K$ é simplesmente conexo e $H$ é conexo, a sequência exata

$$
\pi_{1}(K) \stackrel{\pi_{\sharp}}{\longrightarrow} \pi_{1}(K / H) \stackrel{\Delta}{\longrightarrow} \pi_{0}(H)
$$

implica que $K / H$ é simplesmente conexo. Além disso, como Sol é conexo, a sequência exata

$$
\pi_{1}(\mathrm{Sol} / \mathrm{Sol} \cap H) \stackrel{\Delta}{\longrightarrow} \pi_{0}(\mathrm{Sol} \cap H) \stackrel{i_{0}}{\longrightarrow} \pi_{0}(\mathrm{Sol})
$$

implica que Sol $\cap H$ é conexo, pois $\operatorname{Sol} / \operatorname{Sol} \cap H \approx K / H$ é simplesmente conexo. Concluimos, portanto, que o subgrupo de isotropia $\mathrm{Sol} \cap H$ é discreto e conexo, portanto é trivial. Isso mostra que a ação (5.40) é livre. Disso decorre então que a aplicação $\bar{\beta}_{g H}$, definida em (1.44), é um difeomorfismo entre Sol e $K / H$. Mostremos agora que a métrica $\bar{g}$, definida em (5.34), é Sol-invariante. Pelo Corolário 1.6.6, a métrica $\bar{g}$ é $\overline{\mathrm{Ad}}(H)$-ivariante se, e somente se,

$$
\bar{g}\left(\overline{\operatorname{Ad}}_{Y} X, Z\right)+\bar{g}\left(X, \overline{\operatorname{Ad}}_{Y} Z\right)=0 .
$$

Matricialmente, a igualdade (5.43) é equivalente a

$$
\bar{g} \cdot \overline{\operatorname{Ad}}_{Y}=-\overline{\mathrm{Ad}}^{*} \cdot \bar{g}
$$

De (5.36), temos

$$
\overline{\operatorname{Ad}}_{Y}=\left(\begin{array}{ccc}
0 & 0 & 0 \\
0 & 0 & 1 \\
-1 & 0 & 0
\end{array}\right)
$$

Assim,

$$
\begin{aligned}
\bar{g} \cdot \overline{\operatorname{Ad}}_{Y} & =\left(\begin{array}{ccc}
0 & 1 & 0 \\
1 & 0 & 0 \\
0 & 0 & 1
\end{array}\right)\left(\begin{array}{ccc}
0 & 0 & 0 \\
0 & 0 & 1 \\
-1 & 0 & 0
\end{array}\right)=\left(\begin{array}{ccc}
0 & 0 & 1 \\
0 & 0 & 0 \\
-1 & 0 & 0
\end{array}\right) \\
& =\left(\begin{array}{ccc}
0 & 0 & 1 \\
0 & 0 & 0 \\
0 & -1 & 0
\end{array}\right)\left(\begin{array}{lll}
0 & 1 & 0 \\
1 & 0 & 0 \\
0 & 0 & 1
\end{array}\right)=-\overline{\operatorname{Ad}}_{Y}^{*} \cdot \bar{g} .
\end{aligned}
$$

Portanto, pelo Corolário 1.6.6, a matriz $\bar{g}$, definida em todo o espaço $K / H$ por translações à esquerda, é Sol-invariante. 
Corolário 5.4.2 $\operatorname{dim}(\mathrm{Iso}(\mathrm{Sol}))=4$.

Demonstração. Consideremos o homomorfismo de grupos de Lie

$$
\phi: K \rightarrow \operatorname{Iso}(K / H)
$$

definido por

$$
\phi(g)=\bar{L}_{g}: K / H \rightarrow K / H,
$$

para todo $g \in K$. O núcleo de $\phi$ é o subgrupo normal fechado de $K$ dado por

$$
\operatorname{ker} \phi=\bigcap_{g \in K} g H g^{-1} \text {. }
$$

Note que ker $\phi$ é um subgrupo de $H$. Como $H$ é conexo e $\operatorname{dim}(H)=1$, segue que $\operatorname{ker} \phi=H$ ou ker $\phi$ é discreto. Se $\operatorname{ker} \phi=H$ então $H$ é normal em $K$. Mas isso não ocorre pois a álgebra de Lie $\mathfrak{h}$ de $H$ não é um ideal em $\mathfrak{k}$. Assim, $\operatorname{ker} \phi$ é um subgrupo discreto de $K$ e, consequentemente, $\operatorname{dim}(\operatorname{ker} \phi)=0$. Portanto, o isomorfismo

$$
K / \operatorname{ker} \phi \simeq \phi(K)
$$

implica que $\operatorname{dim}(\operatorname{Iso}(K / H)) \geq 4$, pois $\operatorname{dim}(K)=4$. Como a métrica $\bar{g}$ não é de curvatura seccinal constante, o Lema 5.1.2 implica que $\operatorname{dim}(\operatorname{Iso}(K / H))=$ 4. Como Sol e $K / H$ estão identificados, através da métrica $\bar{g}$, segue a afirmação.

A partir de agora, consideremos o grupo de Lie Sol identificado com a variedade Lorentziana $K / H$, como no Teorema 5.4.1. O subespaço m gerado pelos campos vetoriais $T, X, Z$ é um subespaço de $\mathfrak{k}$ complementar a $\mathfrak{h}$, i.e., $\mathfrak{k}=\mathfrak{h} \oplus \mathfrak{m}$. Note que os campos vetoriais $T, X, Z$ geram a álgebra de Lie do grupo Sol, $\operatorname{logo}[\mathfrak{m}, \mathfrak{m}] \subseteq \mathfrak{m}$. Assim, identificaremos canonicamente o espaço tangente $T_{1 H}(K / H)$ com o subespaço $\mathfrak{m}$. Denotaremos também por $\bar{g}$ a métrica Lorentziana em $K / H$ que, em $\mathfrak{m}$, coincide com a métrica (5.34).

Denotemos por $\bar{\nabla}$ a conexão de Levi-Civita de $(K / H, \bar{g})$ e consideremos a aplicação linear $\lambda: \mathfrak{m} \rightarrow \mathfrak{g l}(\mathfrak{m})$ correspondente a $\nabla$, dada pelo Lema 2.6.2. De (2.69), segue que $\lambda$ é dada por:

$$
\langle\lambda(X) Y, Z\rangle=\frac{1}{2}(\langle[X, Y], Z\rangle-\langle[X, Z], Y\rangle-\langle[Y, Z], X\rangle),
$$

para quaisquer $X, Y, Z \in \mathfrak{m}$. Denotando por $\bar{R}$ o tensor de curvatura de $\bar{\nabla}$, temos a seguinte: 
Proposição 5.4.3 Para quaisquer $X, Y \in \mathfrak{m}$, temos:

$$
\bar{R}_{1 H}(X, Y)=[\lambda(X), \lambda(Y)]-\lambda([X, Y]) \in \mathfrak{g l}(\mathfrak{m}) .
$$

Demonstração. Segue diretamente da Proposição 2.6.5, lembrando que $[\mathfrak{m}, \mathfrak{m}] \subseteq \mathfrak{m}$.

\subsubsection{Imersões isométricas no grupo Sol}

Seguindo a notação da Seção 3.4, consideremos a $G$-estrutura $\bar{P}$ em $\mathrm{FR}_{\mathfrak{m}}^{\mathrm{o}}(T(K / H))$ dada por:

$$
\bar{P}=\left\{\mathrm{d} \bar{L}_{g}(1 H): g \in K\right\},
$$

onde

$$
G=\overline{\operatorname{Ad}}(H) \subset \mathrm{O}(\mathfrak{m}) .
$$

Mais precisamente, $G$ é o subgrupo de Lie de $\mathrm{O}(\mathfrak{m})$ dado por:

$$
G=\left\{\mathrm{d} \bar{L}_{h}(1 H): h \in H\right\} .
$$

Denotando por $\bar{\nabla}$ a conexão de Levi-Civita de $(K / H, \bar{g})$, temos a seguinte:

Proposição 5.4.4 A terna $(K / H, \bar{\nabla}, \bar{P})$ é infinitesimalmente homogênea.

Demonstração. Isso é uma consequência direta do Lema 4.4 .6 pois, neste caso, a terna $(K / H, \bar{\nabla}, \bar{P})$ é $G$-homogênea. De forma mais explícita, o tensor de curvatura $R$ e a inner torsion $\mathfrak{I}^{\bar{P}}$ da $G$-estrutura (5.45) são dados por

$$
\bar{R}_{1 H}(X, Y)=[\lambda(X), \lambda(Y)]-\lambda([X, Y])
$$

e

$$
\mathfrak{I}_{1 H}^{\bar{P}}(X)=(\mathfrak{q} \circ \lambda)(X),
$$

para quaisquer $X, Y \in \mathfrak{m}$ (cf. Proposição 5.4.3 e Proposição 3.4.1), onde $\lambda$ é dada por $(5.44)$ e $\mathfrak{q}: \mathfrak{g l}(\mathfrak{m}) \rightarrow \mathfrak{g l}(\mathfrak{m}) / \overline{\operatorname{ad}}(\mathfrak{h})$ denota a aplicação quociente.

Consideremos agora os seguintes objetos: uma variedade Riemanniana $(M, g)$ de dimensão $2, \nabla$ a conexão de Levi-Civita de $(M, g), E=T M \times \mathbb{R}$ o fibrado vetorial trivial sobre $M$ munido de uma métrica semi-Riemanniana $g^{E}$ e denotemos por $\nabla^{E}$ uma conexão em $E$ compatível com $g^{E}$. Dado uma seção diferenciável simétrica $\alpha$ do fibrado vetorial $\operatorname{Lin}_{2}(T M ; E)$, denotemos por $\widehat{g}$ a métrica semi-Riemanniana no fibrado $\widehat{E}=T M \oplus E$ dada pela soma 
direta ortogonal de $g$ e $g^{E}$, e consideremos a conexão $\widehat{\nabla}$ em $\widehat{E}$ cujas componentes são $\nabla, \nabla^{E}$ e $\alpha$. Além disso, fixemos $p_{0} \in \mathrm{FR}_{\mathfrak{m}}^{\mathrm{o}}(\widehat{E})$ e consideremos a $G$-estrutura

$$
\widehat{P}=p_{0} \cdot G=\left\{p_{0} \circ g: g \in G\right\} .
$$

Mais precisamente, $\widehat{P}$ é dada por:

$$
\widehat{P}=\left\{p_{0} \circ \mathrm{d} L_{h}(1 H): h \in H\right\} .
$$

Com a notação fixada acima, temos o seguinte:

Teorema 5.4.5 Suponhamos que, para todo $x \in M$, e para toda aplicação $\sigma: \widehat{E}_{x} \rightarrow \mathfrak{m}$ que preserva $G$-estrutura, valem as seguintes condições:

(a) $\widehat{R}_{x}$ é $\sigma$-relacionado com $\bar{R}_{1 H}$, onde $\widehat{R}$ denota o tensor de curvatura da conexão $\widehat{\nabla}$,

(b) O seguinte diagrama comuta:

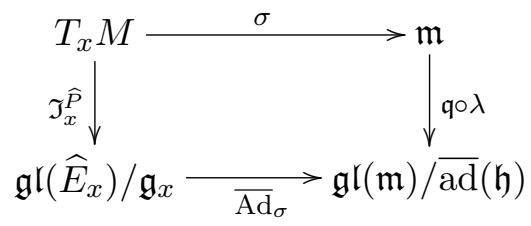

onde $\mathfrak{I}^{\widehat{E}}$ denota a inner torsion da G-estrutura $\widehat{P}$. Então, para quaisquer $x_{0} \in M$ e $y_{0} \in K / H$, e para toda aplicação linear $\sigma_{0}: \widehat{E}_{x_{0}} \rightarrow T_{y_{0}}(K / H)$ que preserva $G$-estrutura, existe uma imersão isométrica $(f, S)$, cujo dominio é uma vizinhança de $x_{0}$ em $M$, tal que $f\left(x_{0}\right)=y_{0}$ e $\mathrm{d} f_{x_{0}} \oplus S_{x_{0}}=\sigma_{0}$.

Demonstração. A condição (a) claramente implica as equações (4.25) e (4.26), e a comutatividade do diagrama (5.46) implica a condição (4.28). Assim, a conclusão segue do Teorema 4.5.2 


\section{Capítulo 6}

\section{Rigidez Isométrica}

\subsection{Introdução}

Sejam $M^{n}, \bar{M}^{n+k}$ variedades Riemannianas. Duas imersões isométricas $f, g: M \rightarrow \bar{M}$ são chamadas congruentes se existe uma isometria $\sigma: \bar{M} \rightarrow \bar{M}$ tal que $g=\sigma \circ f$. Dizemos que uma imersão isométrica $f: M \rightarrow \bar{M}$ é rígida se $f$ é congruente com qualquer outra imersão isométrica.

O primeiro resultado importante em rigidez isométrica é um teorema de Allendoerfer (ver [1]), que garante rigidez local no caso em que $\bar{M}$ é uma variedade Riemanniana de curvatura seccional constante $Q_{c}^{n+k}$. Mais precisamente,

Teorema 6.1.1 Seja $f: M^{n} \rightarrow Q_{c}^{n+k}$ uma imersão isométrica, com $n \geq 3$, tal que o número tipo $\tau_{f}$ de $f$ satisfaz $\tau_{f}(x) \geq 3$, para todo $x \in M$. Então, $f$ é rígida.

Uma prova do Teorema 6.1.1, fazendo uso da teoria de formas bilineares flat, pode ser encontrada em [7]. Este resultado generaliza um resultado clássico de Beez-Killing para hipersuperfícies (ver, por exemplo, [18], [29]). Uma prova do Teorema 6.1.1, para o caso de hipersuperfícies, fazendo uso da teoria de formas diferenciais é devido a E. Cartan.

Outros resultados em rigidez isométrica podem ser considerados quando a subvariedade satisfaz condições mais fortes, como compacidade, completude, curvatura média constante, fibrado normal flat, dentre outras (ver, por exemplo, [2], [5], [8]).

O objetivo deste Capítulo é provar um teorema semelhante ao Teorema 6.1.1 para o caso de imersões isométricas que preservam $G$-estrutura. Mais 
precisamente, para o caso de hipersuperfícies em variedades semi-Riemannianas $G$-homogêneas.

\subsection{G-rigidez isométrica}

Fixemos os seguintes objetos: uma variedade semi-Riemanniana $\left(M^{n}, g\right)$ de índice $r$, uma variedade semi-Riemanniana $\left(\bar{M}^{n+k}, \bar{g}\right)$ de índice $\bar{r}$, um fibrado vetorial $E$ sobre $M, \operatorname{com} \operatorname{rank}(E)=k$, munido de uma métrica semi-Riemanniana $g^{E}$ de índice $s$, onde $\bar{r}=r+s$. Seja $G$ um subgrupo de Lie de $\mathrm{O}_{\bar{r}}(n+k)$ e consideremos $G$-estruturas $\widehat{P} \subset \mathrm{FR}^{\mathrm{o}}(\widehat{E})$ e $\bar{P} \subset \mathrm{FR}^{\mathrm{o}}(T \bar{M})$.

Definição 6.2.1 Sejam $\left(f^{1}, S^{1}\right),\left(f^{2}, S^{2}\right)$ imersões isométricas de $M$ em $\bar{M}$ que preservam $G$-estrutura. Dizemos que $\left(f^{1}, S^{1}\right)$ e $\left(f^{2}, S^{2}\right)$ são $G$ congruentes se existe uma isometria $\sigma: \bar{M} \rightarrow \bar{M}$ que preserva $G$-estrutura tal que

$$
f^{2}=\sigma \circ f^{1} \quad \text { e } \quad S^{2}= \pm\left.\mathrm{d} \sigma\right|_{T M_{f^{1}}^{\perp}} \circ S^{1} .
$$

Definição 6.2.2 Dizemos que uma imersão isométrica $(f, S)$ que preserva $G$-estrutura é $G$-rígida se $(f, S)$ é $G$-congruente com qualquer outra imersão isométrica que preserva $G$-estrutura.

O Lema seguinte é consequência da unicidade do Teorema 4.5.2, para o caso em que as imersões isométricas têm os mesmos dados prescritos.

Lema 6.2.3 Sejam $\left(f^{1}, S^{1}\right),\left(f^{2}, S^{2}\right)$ imersões isométricas que preservam $G$-estrutura com os mesmos dados prescritos $\nabla^{E}$ e $\alpha$ (cf. Definição 4.3.1). Se, além disso, $(\bar{M}, \bar{\nabla}, \bar{P})$ é $G$-homogênea então $\left(f^{1}, S^{1}\right)$ e $\left(f^{2}, S^{2}\right)$ são $G$ congruentes.

Demonstração. Dado $x_{0} \in M$, sejam $y_{1}=f^{1}\left(x_{0}\right)$ e $y_{2}=f^{2}\left(x_{0}\right)$. A aplicação linear $\sigma_{0}: T_{y_{1}} \bar{M} \rightarrow T_{y_{2}} \bar{M}$, dada por $\sigma_{0}=L_{x_{0}}^{2} \circ\left(L_{x_{0}}^{1}\right)^{-1}$, é um isomorfismo linear que preserva $G$-estrutura. Assim, como $\bar{M}$ é globalmente homogênea, existe uma isometria $\sigma: \bar{M} \rightarrow \bar{M}$ que preserva $G$-estrutura tal que $\sigma\left(y_{1}\right)=y_{2}$ e $\mathrm{d} \sigma_{y_{1}}=\sigma_{0}$. Consideremos o par $(\tilde{f}, \widetilde{S})$, onde

$$
\tilde{f}=\sigma \circ f^{1} \quad \text { e } \quad \widetilde{S}=\left.\mathrm{d} \sigma\right|_{T M_{f^{1}}^{\perp}} \circ S^{1} .
$$

Mostremos que $(\tilde{f}, \widetilde{S})$ é uma imersão isométrica que preserva $G$-estrutura com os mesmos dados prescritos $\nabla^{E}, \alpha, g^{E}$. Claramente a segunda forma 
fundamental $\alpha^{\tilde{f}}$ da imersão isométrica $\tilde{f}$ é dada por $\alpha^{\tilde{f}}=\left.\mathrm{d} \sigma\right|_{T M_{f^{1}}^{\perp}} \circ \alpha^{f^{1}}$, onde $\alpha^{f^{1}}$ é a segunda forma fundamental de $f^{1}$. O fato de $\sigma: \bar{M} \rightarrow \bar{M}$ ser uma isometria garante que $\widetilde{S}: E \rightarrow T M_{\sigma \circ f^{1}}^{\perp}$ é uma isometria de fibrados vetoriais. Como $S^{1}$ preserva conexão e segunda forma fundamental, temos:

$$
\begin{aligned}
\widetilde{S}(\alpha(X, Y)) & =\left.\mathrm{d} \sigma\right|_{T M_{f^{1}}^{\perp}}(\alpha(X, Y))=\left.\mathrm{d} \sigma\right|_{T M_{f^{1}}^{\perp}}\left(\alpha^{f^{1}}(X, Y)\right) \\
& =\alpha^{\tilde{f}}(X, Y)
\end{aligned}
$$

e

$$
\begin{aligned}
\widetilde{S}\left(\nabla_{X}^{E} \epsilon\right) & =\left.\mathrm{d} \sigma\right|_{T M_{f^{1}}^{\perp}}\left(S^{1}\left(\nabla_{X}^{E} \epsilon\right)\right)=\left.\mathrm{d} \sigma\right|_{T M_{f^{1}}^{\perp}}\left(\nabla_{f^{1} X}^{\perp} S^{1}(\epsilon)\right) \\
& =\nabla_{\sigma \circ f^{1} X}^{\perp} \widetilde{S}(\epsilon)
\end{aligned}
$$

para quaisquer $X, Y \in \boldsymbol{\Gamma}(T M)$ e $\epsilon \in \boldsymbol{\Gamma}(E)$, onde $\nabla_{\sigma \circ f^{1}}^{\perp}$ denota a conexão normal em $T M_{\sigma \circ f^{1}}^{\perp}$. Finalmente, o isomorfismo $\widetilde{L}: \widehat{E} \rightarrow T \bar{M}$, dado por $\widetilde{L}=\mathrm{d} \tilde{f} \oplus \widetilde{S}$, preserva $G$-estrutura, pois é a composta de isomorfismos que preservam $G$-estrutura. Portanto, os pares $\left(f^{2}, S^{2}\right)$ e $(\tilde{f}, \widetilde{S})$ são imersões isométricas que preservam $G$-estrutura com os mesmos dados prescritos $\nabla^{E}$, $\alpha, g^{E}$. Por construção, é fácil ver que, no ponto $x_{0}$, temos

$$
f^{2}\left(x_{0}\right)=\tilde{f}\left(x_{0}\right), \quad \mathrm{d} f_{x_{0}}^{2}=\mathrm{d} \tilde{f}_{x_{0}} \quad \text { e } \quad S_{x_{0}}^{2}=\widetilde{S}_{x_{0}} .
$$

Assim, pelo Teorema 4.5.2, concluímos que $f^{2}=\tilde{f}$ e $S^{2}=\widetilde{S}$, ou seja,

$$
f^{2}=\sigma \circ f^{1} \quad \text { e } \quad S^{2}=\left.\mathrm{d} \sigma\right|_{T M_{f^{1}}^{\perp}} \circ S^{1}
$$

como queríamos.

\subsection{G-rigidez isométrica de hipersuperfícies}

Passaremos agora a considerar o caso em que $\operatorname{dim}(\bar{M})=n+1$.

Lema 6.3.1 Quando $\operatorname{rank}(E)=1$, existe uma única conexão $\nabla^{E}$ em $E$ compatível com a métrica $g^{E}$.

Demonstração. Sejam $\nabla^{1}, \nabla^{2}$ conexões em $E$ compatíveis com $g^{E}$. Fixado $X \in \boldsymbol{\Gamma}(T M)$, consideremos a aplicação

$$
D(X): \boldsymbol{\Gamma}(E) \rightarrow \boldsymbol{\Gamma}(E)
$$


definida por

$$
D(X) \epsilon=\nabla_{X}^{1} \epsilon-\nabla_{X}^{2} \epsilon
$$

para todo $\epsilon \in \boldsymbol{\Gamma}(E)$. Claramente $D(X)$ é $\mathrm{C}^{\infty}(M)$-linear. Além disso, $D(X)$ é anti-simétrica pois, para quaisquer $\epsilon_{1}, \epsilon_{2} \in \Gamma(E)$, temos:

$$
\begin{aligned}
g^{E}\left(D(X) \epsilon_{1}, \epsilon_{2}\right) & =g^{E}\left(\nabla_{X}^{1} \epsilon_{1}-\nabla_{X}^{2} \epsilon_{1}, \epsilon_{2}\right) \\
& =X g^{E}\left(\epsilon_{1}, \epsilon_{2}\right)-g^{E}\left(\epsilon_{1}, \nabla_{X}^{1} \epsilon_{2}\right)-X g^{E}\left(\epsilon_{1}, \epsilon_{2}\right)+g^{E}\left(\epsilon_{1}, \nabla_{X}^{2} \epsilon_{2}\right) \\
& =-g^{E}\left(\epsilon_{1}, D(X) \epsilon_{2}\right) .
\end{aligned}
$$

Agora, $\operatorname{como} \operatorname{rank}(E)=1$, o fato de $D(X)$ ser anti-simétrica implica $D(X)=$ 0 , ou seja, $\nabla_{X}^{1} \epsilon=\nabla_{X}^{2} \epsilon$, para todo $\epsilon \in \Gamma(E)$. Como $X$ é arbitrário, segue que $\nabla^{1}=\nabla^{2}$.

Seja $(f, S)$ uma imersão isométrica de $M^{n}$ em $\bar{M}^{n+1}$ que preserva $G$ estrutura. Fixado um campo normal unitário (local) $N$, temos o operador de Weingarten

$$
A: \boldsymbol{\Gamma}(T M) \rightarrow \boldsymbol{\Gamma}(T M)
$$

associado. Neste caso, a equação de Gauss pode ser escrita como:

$$
\pi^{M}\left(\bar{R}_{\widehat{E}_{x}}(X, Y) Z\right)=(A X \wedge A Y) Z+R_{x}(X, Y) Z,
$$

para quaisquer $x \in M$ e $X, Y, Z \in T_{x} M$ (cf. Exemplo 4.3.5).

Lema 6.3.2 Seja $x \in M$ tal que $\operatorname{rank}\left(A_{x}\right) \geq 2$. Então $\operatorname{ker}\left(A_{x}\right)=T_{\mathrm{o}}(x)$, onde

$$
T_{\mathrm{o}}(x)=\left\{X \in T_{x} M: \bar{R}_{\widehat{E}_{x}}(X, Y)=R_{x}(X, Y), \forall Y \in T_{x} M\right\} .
$$

Demonstração. A inclusão $\operatorname{ker}\left(A_{x}\right) \subset T_{\mathrm{o}}(x)$ segue diretamente de (6.4). Reciprocamente, seja $X \in T_{\mathrm{o}}(x)$. Como $\operatorname{rank}\left(A_{x}\right) \geq 2$, existe $Y \in T_{x} M$ tal que $A Y \neq 0$ e $\langle A X, A Y\rangle=0$. Como $X \in T_{0}(x)$ temos $A X \wedge A Y=0$. Assim,

$$
0=(A X \wedge A Y) A X=\langle A X, A X\rangle A Y-\langle A Y, A X\rangle A X .
$$

Isso implica que $\langle A X, A X\rangle=0$ e, portanto, $A X=0$, mostrando a inclusão $T_{\mathrm{o}}(x) \subset \operatorname{ker}\left(A_{x}\right)$. 
Lema 6.3.3 Sejam $\left(f^{1}, S^{1}\right),\left(f^{2}, S^{2}\right)$ imersões isométricas que preservam $G$-estrutura. Se $\operatorname{rank}\left(A_{x}^{1}\right) \geq 3$, para todo $x \in M$, então $A^{1}= \pm A^{2}$.

Demonstração. Mostremos inicialmente que $\operatorname{ker}\left(A_{x}^{1}\right)=\operatorname{ker}\left(A_{x}^{2}\right)$, para todo $x \in M$. Para isso, suponha que exista $x \in M \operatorname{com} \operatorname{rank}\left(A_{x}^{2}\right) \leq 1$. Isso implica que $A^{2} X \wedge A^{2} Y=0$, para quaisquer $X, Y \in T_{x} M$ e, portanto, $\operatorname{dim}\left(T_{\mathrm{o}}(x)\right)=n$, contradizendo o fato de que $\operatorname{rank}\left(A_{x}^{1}\right) \geq 3$. Logo, devemos ter $\operatorname{rank}\left(A_{x}^{2}\right) \geq 2$, para todo $x \in M$. Pelo Lema 6.3.2, temos:

$$
\operatorname{ker}\left(A_{x}^{2}\right)=T_{\mathrm{o}}(x)=\operatorname{ker}\left(A_{x}^{1}\right),
$$

para todo $x \in M$. Mostremos agora que, para quaisquer $x \in M$ e $X \in T_{x} M$, os vetores $A^{1} X, A^{2} X$ são linearmente dependentes. De fato, suponha que exista $X \in T_{x} M$ tal que $A^{1} X, A^{2} X$ são linearmente independentes. Como $\operatorname{rank}\left(A_{x}^{1}\right) \geq 3$, segue que $\operatorname{Im}\left(A_{x}^{1}\right) \not \subset \operatorname{span}\left\{A^{1} X, A^{2} X\right\}$. Assim, existe $Y \in$ $T_{x} M$ tal que os vetores $A^{1} X, A^{1} Y, A^{2} X$ são linearmente independentes, ou seja, $A^{1} X \wedge A^{1} Y \wedge A^{2} X \neq 0$. Por outro lado, de (6.5) temos que $A^{1} X \wedge A^{1} Y=$ $A^{2} X \wedge A^{2} Y$, pois o valor

$$
\bar{R}_{\widehat{E}_{x}}(X, Y)-R_{x}(X, Y)
$$

não depende da imersão isométrica. Assim, $A^{2} X \wedge A^{2} Y \wedge A^{2} X \neq 0$, o que é uma contradição. Assim, para cada $X \in T_{x} M$, existe uma constante $c=c(X)$ tal que $A^{1} X=c A^{2} X$. Agora, dados $X_{1}, X_{2} \in T_{x} M$, existem constantes $c_{1}, c_{2}, c_{3}$ tais que:

$$
\begin{aligned}
A^{1} X_{1} & =c_{1} A^{2} X_{1}, \\
A^{1} X_{2} & =c_{2} A^{2} X_{2}, \\
A^{1}\left(X_{1}+X_{2}\right) & =c_{3} A^{2}\left(X_{1}+X_{2}\right) .
\end{aligned}
$$

Temos, então:

$$
\left(c_{1}-c_{3}\right) A^{2} X_{1}+\left(c_{2}-c_{3}\right) A^{2} X_{2}=0 .
$$

Como $A_{x}^{2}$ é injetora em $\left(T_{\mathrm{o}}(x)\right)^{\perp}$, segue que $c_{1}=c_{2}=c_{3}$, para $X_{1}, X_{2} \in$ $\left(T_{\mathrm{o}}(x)\right)^{\perp}$. Claramente esta equação também vale para $X_{1}, X_{2} \in T_{\mathrm{o}}(x)$. Assim, $A^{1} X=c A^{2} X$ para alguma constante $c$ independente de $X$. Finalmente, como

$$
A^{1} X \wedge A^{1} Y=c^{2} A^{2} X \wedge A^{2} Y,
$$

temos $c= \pm 1$. Isso mostra que $A^{1}= \pm A^{2}$. 
Corolário 6.3.4 Sejam $\left(f^{1}, S^{1}\right),\left(f^{2}, S^{2}\right)$ imersões isométricas que preservam $G$-estrutura tal que $\operatorname{rank}\left(A_{x}^{1}\right) \geq 3$, para todo $x \in M$. Suponha, além disso, que $M$ é conexa e orientável. Então, escolhendo apropriadamente os campos normais unitários para $f^{1}$ e $f^{2}$, temos $A^{1}=A^{2}$.

Demonstração. Sejam $N_{1}$ e $N_{2}$ campos normais unitários globalmente definidos em $M$ para $f^{1}$ e $f^{2}$, respectivamente. Denotemos por $M^{+}$(resp. $M^{-}$) o conjunto dos pontos $x \in M$ para os quais $A^{1}=A^{2}$ (resp. $A^{1}=-A^{2}$ ). Pelo Lema 6.3.3, $M$ é união disjunta de $M^{+}$e $M^{-}$. Além disso, $M^{+}$e $M^{-}$ são ambos abertos e fechados em $M$. Como $M$ é conexa temos $M=M^{+}$ou $M=M^{-}$. Se $M=M^{+}$o Corolário está provado. Se $M=M^{-}$, trocamos $N_{2}$ por $-N_{2}$.

Teorema 6.3.5 Seja $(f, S)$ uma imersão isométrica de $M^{n}$ em $\bar{M}$ que preserva $G$-estrutura tal que $\operatorname{rank}\left(A_{x}\right) \geq 3$, para todo $x \in M$. Suponha, além disso, que $M$ é conexa e orientável, e $(\bar{M}, \bar{\nabla}, \bar{P})$ é $G$-homogênea. Então $(f, S)$ é $G$-rígida.

Demonstração. Denotemos por $\left(f^{1}, S^{1}\right)=(f, S)$ e seja $\left(f^{2}, S^{2}\right)$ outra imersão isométrica que preserva $G$-estrutura. Fixemos campos normais unitários $N_{1}, N_{2}$, globalmente definidos em $M$, para $f^{1}$ e $f^{2}$, respectivamente. Definimos um isomorfismo de fibrados vetoriais $T: T M_{f^{1}}^{\perp} \rightarrow T M_{f^{2}}^{\perp}$, dado por

$$
T=S^{2} \circ\left(S^{1}\right)^{-1} .
$$

Mais precisamente, $T$ é uma isometria de fibrados vetoriais. Por outro lado, como $\operatorname{rank}\left(A_{x}^{1}\right) \geq 3$, para todo $x \in M$, segue do Lema 6.3.3 que $A^{1}= \pm A^{2}$. Isso implica que $\alpha^{f^{2}}=I \circ \alpha^{f^{1}}$, onde $I: T M_{f^{1}}^{\perp} \rightarrow T M_{f^{2}}^{\perp}$ é uma isometria de fibrados vetoriais. $\operatorname{Como} \operatorname{rank}\left(T M_{f^{i}}^{\perp}\right)=1, i=1,2$, segue que $I= \pm T$. Analisemos, então, os seguintes casos:

Caso 1. Se $I=T$, temos o seguinte diagrama:

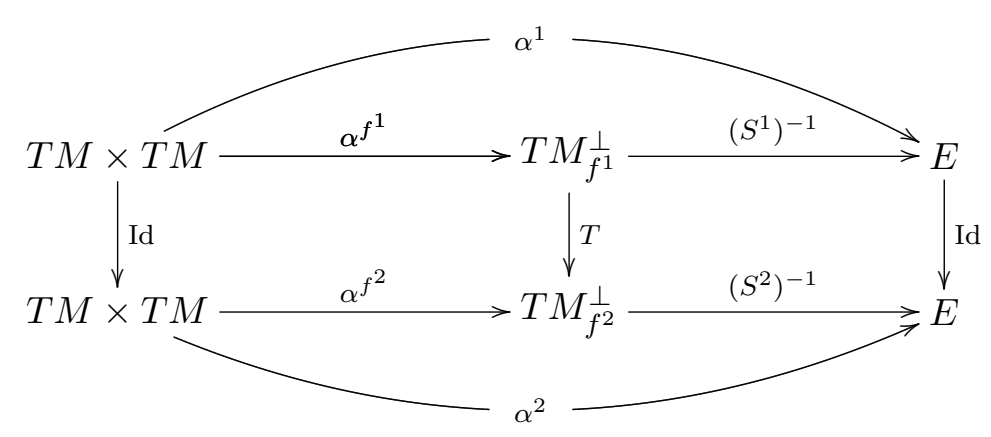


A comutatividade do quadrado à direita em (6.6) implica que $\alpha^{1}=\alpha^{2}$. Portanto, $\left(f^{1}, S^{1}\right)$ e $\left(f^{2}, S^{2}\right)$ são imersões isométricas que preservam $G$-estrutura com os mesmos dados prescritos $\nabla^{E}, \alpha, g^{E}$, onde $\alpha=\alpha^{1}=\alpha^{2}$. Assim, pelo Lema 6.2.3, existe uma isometria que preserva $G$-estrutura $\sigma: \bar{M} \rightarrow \bar{M}$ tal que $f^{2}=\sigma \circ f^{1}$ e $S^{2}=\left.\mathrm{d} \sigma\right|_{T M_{f^{1}}^{\perp}} \circ S^{1}$, ou seja, $(f, S)$ é $G$-rígida.

Caso 2. Suponha $I=-T$. Pelo diagrama (6.6), concluimos que $\alpha^{1}=-\alpha^{2}$. Consideremos a imersão isométrica que preserva $G$-estrutura $\left(f^{1}, \widetilde{S}^{1}\right)$, onde $\widetilde{S}^{1}=-S^{1}$. É fácil ver que os dados prescritos de $\left(f^{1}, \widetilde{S}^{1}\right)$ são $\nabla^{E},-\alpha^{1}, g^{E}$ que, por sua vez, são os mesmos dados prescritos de $\left(f^{2}, S^{2}\right)$. Portando, pelo Lema 6.2.3, existe uma isometria que preserva $G$-estrutura $\sigma: \bar{M} \rightarrow \bar{M}$ tal que $f^{2}=\sigma \circ f^{1}$ e $S^{2}=-\left.\mathrm{d} \sigma\right|_{T M_{f^{1}}^{\perp}} \circ S^{1}$, provando também, neste caso, que $(f, S)$ é $G$-rígida.

Veremos a seguir o problema de rigidez isométrica em casos particulares, onde se conhece, explicitamente, a $G$-estrutura considerada.

\section{4 $G$-rigidez isométrica de hipersuperfícies em pro- dutos $Q_{c}^{n} \times \mathbb{R}$ e em 3-variedades}

Em alguns casos, o Teorema 6.3.5 pode ser obtido sem a hipótese de que $\operatorname{rank}\left(A_{x}^{1}\right) \geq 3$, para todo $x \in M$. Isso é um fato que depende da escolha das $G$-estruturas consideradas em $\widehat{E}$ e $\bar{M}$, e do próprio ambiente $\bar{M}$ considerado.

Antes de exemplificar tais situações, faremos algumas considerações. Sejam $\left(f^{1}, S^{1}\right),\left(f^{2}, S^{2}\right)$ imersões isométricas que preservam $G$-estrutura. Denotemos por $\nabla$ a conexão de Levi-Civita de $(M, g)$ e $\nabla^{E}$ a única conexão em $E$ que é compatível com $g^{E}$. Se $\widehat{\nabla}^{i}$ denota a conexão em $\widehat{E}$ cujas componentes são $\nabla, \nabla^{E}, \alpha^{i}, i=1,2$, temos:

$$
\widehat{\nabla}_{X}^{i}(Y, \eta)=\nabla_{X} Y-\eta A^{i} X+\nabla_{X}^{E} \eta+\alpha^{i}(X, Y)
$$

para quaisquer $X, Y \in \boldsymbol{\Gamma}(T M), \eta \in \boldsymbol{\Gamma}(E)$ e $\mathrm{i}=1,2$ (cf. (4.2)). Fixado $X \in$ $\boldsymbol{\Gamma}(T M)$, consideremos a aplicação linear:

$$
D(X): \boldsymbol{\Gamma}(\widehat{E}) \rightarrow \boldsymbol{\Gamma}(\widehat{E})
$$

definida por

$$
D(X)=\widehat{\nabla}_{X}^{1} \epsilon-\widehat{\nabla}_{X}^{2} \epsilon
$$

para todo $\epsilon \in \boldsymbol{\Gamma}(\widehat{E})$. Dados $Y \in \boldsymbol{\Gamma}(T M)$ e $\eta \in \boldsymbol{\Gamma}(E)$, temos:

$$
\begin{aligned}
D(X) Y & =\alpha^{1}(X, Y)-\alpha^{2}(X, Y), \\
D(X) \eta & =\eta\left(A^{2} X-A^{1} X\right) .
\end{aligned}
$$


Assim,

$$
D(X)(Y, \eta)=\left(\eta\left(A^{2} X-A^{1} X\right), \alpha^{1}(X, Y)-\alpha^{2}(X, Y)\right),
$$

para quaisquer $Y \in \boldsymbol{\Gamma}(T M)$ e $\eta \in \boldsymbol{\Gamma}(E)$.

\subsection{1 $G$-rigidez isométrica de hipersuperfícies em $Q_{c}^{n} \times \mathbb{R}$}

Consideremos o caso em que $\bar{M}=Q_{c}^{n} \times \mathbb{R}$ é o produto ortogonal de uma variedade Riemanniana de curvatura seccional constante $Q_{c}^{n}$ por $\mathbb{R}$. Neste caso, a $G$-estrutura $\widehat{P} \subset \mathrm{FR}^{\circ}(\widehat{E})$ é dada por

$$
\widehat{P}=\operatorname{FR}^{\mathrm{o}}\left(\widehat{E} ; e_{n+1},(T, \nu)\right),
$$

onde $\left\{e_{1}, \ldots, e_{n+1}\right\}$ denota a base canônica de $\mathbb{R}^{n+1}, T \in \boldsymbol{\Gamma}(T M)$ e $\nu \in$ $\mathrm{C}^{\infty}(M)$ são tais que $\|T\|^{2}+\nu^{2}=1$ e $G \simeq \mathrm{O}(n) \times \mathrm{O}(1)$ (cf. Exemplo 4.5.6).

Considerando a $G$-estrutura $\bar{P}$ em $\bar{M}$, como no Exemplo 4.5.6, temos o seguinte:

Teorema 6.4.1 Seja $(f, S)$ uma imersão isométrica de $M$ em $Q_{c}^{n} \times \mathbb{R}$ que preserva $G$-estrutura. Se o conjunto $\{x \in M: \nu(x) \neq 0\}$ é denso em $M$, então $(f, S)$ é G-rígida.

Demonstração. Seja $\left(f^{2}, S^{2}\right)$ outra imersão isométrica que preserva $G$ estrutura. Denotemos $\left(f^{1}, S^{1}\right)=(f, S)$ e seja $F$ o subfibrado vetorial de $\widehat{E}$ gerado pelo par $(T, \nu)$. A hipótese de que a derivada covariante $\widehat{\nabla}^{i}$ de seções de $F$ são seções de $F, i=1,2$, implica que

$$
\widehat{\nabla}_{X}^{i}(T, \nu) \in \boldsymbol{\Gamma}(F), \quad i=1,2,
$$

ou seja,

$$
\widehat{\nabla}_{X}^{i}(T, \nu)=\phi_{i}(T, \nu), \quad i=1,2
$$

onde $\phi \in C^{\infty}(M)$. Como $\|(T, \nu)\|=1$ segue que $\phi_{i}=0, i=1,2$. Assim, de (6.7), obtemos

$$
D(X)(T, \nu)=0
$$

e, como consequência,

$$
\nu\left(A^{2} X-A^{1} X\right)=0,
$$

para todo $X \in \boldsymbol{\Gamma}(T M)$. Como o conjunto dos pontos $x \in M$ tal que $\nu(x) \neq 0$ é denso em $M$, concluimos que

$$
A^{1} X=A^{2} X
$$

para todo $X \in \boldsymbol{\Gamma}(T M)$. Isso implica que $\alpha^{1}= \pm \alpha^{2}$ e, pelo Lema 6.2.3, concluimos que $\left(f^{1}, S^{1}\right)$ e $\left(f^{2}, S^{2}\right)$ são $G$-congruentes. Portanto, $(f, S)$ é $G$-rígida. 


\subsubsection{G-rigidez isométrica de superfícies em 3-variedades}

Consideremos o caso em que $\bar{M}$ é uma variedade Riemanniana homogênea orientável de dimensão 3, cujo grupo de isometrias tem dimensão 4. Neste caso, a $G$-estrutura $\widehat{P}$ de $\widehat{E}$ é dada por:

$$
\widehat{P}=\mathrm{FR}_{+}^{\mathrm{o}}\left(\widehat{E} ; e_{1},(T, \nu)\right),
$$

onde $\left\{e_{1}, \ldots, e_{3}\right\}$ denota a base canônica de $\mathbb{R}^{3}, T \in \boldsymbol{\Gamma}(T M)$ e $\nu \in \mathrm{C}^{\infty}(M)$ são tais que $\|T\|^{2}+\nu^{2}=1$ e $G=\mathrm{SO}\left(3 ; e_{1}\right) \simeq \mathrm{SO}(2)$ (cf. Exemplo 4.5.8).

Considerando a $G$-estrutura $\bar{P}$ em $\bar{M}$, como no Exemplo 4.5.8, temos o seguinte:

Teorema 6.4.2 Seja $(f, S)$ uma imersão isométrica de $M$ em $\bar{M}$ que preserva $G$-estrutura. Se o conjunto $\{x \in M: \nu(x) \neq 0\}$ é denso em $M$, então $(f, S)$ é $G$-rígida.

Demonstração. Denotemos por $\left(f^{1}, S^{1}\right)=(f, S)$. A inner torsion $\mathfrak{I}^{\widehat{P}}$ da $G$-estrutura $\widehat{P}$ é dada por

$$
\mathfrak{I}_{x}^{\widehat{P}}(X)=\widehat{\nabla}_{X}^{1}(T, \nu)
$$

para quaisquer $x \in M$ e $X \in T_{x} M$ (cf. Exemplo 3.3.12). Se $\left(f^{2}, S^{2}\right)$ é outra imersão isométrica que preserva $G$-estrutura, temos:

$$
\mathfrak{I}_{x}^{\widehat{P}}(X)=\widehat{\nabla}_{X}^{2}(T, \nu)
$$

para quaisquer $x \in M$ e $X \in T_{x} M$. De (6.8) e (6.9), segue que $D(X)(T, \nu)=$ 0 e, portanto,

$$
\nu\left(A^{2} X-A^{1} X\right)=0,
$$

para todo $X \in \boldsymbol{\Gamma}(T M)$. Como o conjunto dos pontos $x \in M$ tais que $\nu(x) \neq 0$ é denso em $M$, concluimos que

$$
A^{1} X=A^{2} X
$$

para todo $X \in \boldsymbol{\Gamma}(T M)$. Isso implica que $\alpha^{1}= \pm \alpha^{2}$ e, pelo Lema 6.2.3, concluimos que $\left(f^{1}, S^{1}\right)$ e $\left(f^{2}, S^{2}\right)$ são $G$-congruentes. Portanto, $(f, S)$ é $G$-rígida.

Observação 6.4.3 O Teorema 6.4.2 também vale no caso em que $\bar{M}$ é o grupo de Heisenberg Lorentziano. De fato, a $G$-estrutura $\widehat{P}$ considerada é a mesma. Assim, podemos concluir que toda imersão isométrica $(f, S)$ de $M$ em Nil que preserva $G$-estrutura é $G$-rígida. 


\section{Referências Bibliográficas}

[1] C. B. Allendoerfer, Rigidity for spaces de class greater than one, Amer. J. of Math. 61 (1939), 633-644.

[2] J. L. M. Barbosa, M. Dajczer, L. P. Jorge, Rigidity of minimal submanifolds in space forms, Math. Ann. 267 (1984), 433-437.

[3] W. M. Boothby, An Introduction to Differentiable Manifolds and Riemannian Geometry, Mathematics, vol. 120, Academic Press, San Diego, 1986.

[4] M. P. do Carmo, Geometria Riemanniana, Projeto Euclides, Instituto de Matemática Pura e Aplicada, Rio de Janeiro, 1988.

[5] M. P. do Carmo, M. Dajczer, Conformal Rigidity, Amer. J. of Math. 109 (1987), 963-985.

[6] S. S. Chern, The geometry of G-structures, Bull. Amer. Math. Soc. 72, (1966), 167-219.

[7] M. Dajczer, Submanifolds and Isometric Immersions, Mathematics Lecture Series, Vol. 13, Publish or Perish, 1990.

[8] M. Dajczer, D. Gromoll, Rigidity of complete Euclidean hypersurfaces, J. Diff. Geometry 31 (1990), 401-416.

[9] B. Daniel, Isometric immersions into $\mathbb{S}^{n} \times \mathbb{R}$ and $\mathbb{H}^{n} \times \mathbb{R}$ and applications to minimal surfaces, to appear in Trans. Amer. Math. Soc.

[10] B. Daniel, Isometric immersions into 3-dimensional homogeneous manifolds, Comment. Math. Helv. 82, (2007), 87-131.

[11] S. Dumitrescu, A. Zeghib, Géométries Lorentziennes de Dimension 3: Classification et complétude, preprint, 2007 (arXiv:math/0703846v2). 
[12] M. Guediri, Sur la complétude des pseudo-métriques invariantes à gauche sur les groupes de Lie nilpotens, Rend. Sem. Mat. Univ. Politec. Torino, 52(4), (1994), 371-376.

[13] M. Guediri, L. Lafontaine, Sur la complétude des variétés pseudoRiemanniennes, Journal of Geometry and Physics, 15, (1995), 150-158.

[14] S. Helgason, Differential Geometry, Lie Groups, and Symmetric Spaces, Graduate Studies in Mathematics, vol. 34, American Mathematical Society, Rhode Island, 2001.

[15] D. Husemoller, Fibre Bundles, McGraw-Hill, Inc., New York, 1966.

[16] S. Kobayashi, Transformation Groups in Differential Geometry, Classics in Mathematics, Springer-Verlag, Berlin, 1995.

[17] S. Kobayashi, K. Nomizu. Foundations of Differential Geometry, Vol. 1, Wiley Classics Library, New York, 1991.

[18] S. Kobayashi, K. Nomizu. Foundations of Differential Geometry, Vol. 2, Wiley Classics Library, New York, 1996.

[19] S. Kobayashi, Theory of Connections, Ann. Mat. Pura Appl. (4) 43 (1957), 119-194.

[20] J. Milnor, Curvatures of left invariant metrics on Lie groups, Advances in Mathematics 21 (1976), 293-329.

[21] K. Nomizu, Left-invariant Lorentz metrics on Lie groups, Osaka J. Math. 16 (1979), 143-150.

[22] K. Nomizu, T. Sasaki, Affine Differential Geometry, Cambridge Tracts in Mathematics, vol. 111, Cambridge University Press, New York, 1994.

[23] B. O'Neill, Semi-Riemannian Geometry with Applications to Relativity, Academic Press, New York, 1983.

[24] H. Osborn, Vector Bundles, Foundations and Stiefel-Whitney Classes, Vol. 1, Mathematics, Academic Press, 1982.

[25] V. Patrangenaru, Classifying 3 and 4 dimensional homogeneous Riemannian manifolds by Cartan triples, Pacific Journal of Mathematics, 173, No. 2, (1996), 511-532. 
[26] P. Piccione, D. V. Tausk, An existence theorem for G-structure preserving affine immersions, preprint 2007, to appear in Indiana Univ. Math J.

[27] P. Piccione, D. V. Tausk, The Theory of Connections and G-structures: Applications to Affine and Isometric Immersions, XIV Escola de Geometria Diferencial, Salvador, Bahia, 2006.

[28] N. Rahmani, S. Rahamani, Lorentzian geometry of the Heisenberg group, Geom. Dedicata, 118, (2006), 133-140.

[29] P. J. Ryan, Homogeneity and some curvature conditions for hypersurfaces, Tôhoku Math. Journ. 21 (1969), 363-388.

[30] P. Scott, The Geometries of 3-manifolds, Bull. London Math. Soc., 15, (1983), 401-487.

[31] I. M. Singer, Infinitesimally homogeneous spaces, Comm. Pure Appl. Math., 13, (1960), 685-697.

[32] S. Sternberg, Lectures on Differential Geometry, Prentice-Hall, Englewood Cliffs, N. J., 1964.

[33] K. Tenenblat, On isometric immersions of Riemannian manifolds, Bol. Soc. Brasil. Mat., 2 (1971), No. 2, 23-36.

[34] W. Thurston, The geometry and topology of 3-manifolds, Princeton University Press, (1983).

[35] V. S. Varadarajan, Lie Groups, Lie Algebras, and Their Representations, Graduate Texts in Mathematics, vol. 102, Springer-Verlag, New York, 1984.

[36] F. W. Warner, Foundations of Differentiable Manifolds and Lie Groups, Graduate Texts in Mathematics, vol. 94, Springer-Verlag, New York, 1983.

[37] J. A. Wolf, Spaces of Constant Curvature, Publish or Perish, Inc., Wilmington, 1984. 